\title{
Regulation of binding of HP1 associated complexes to chromatin and their role in transcription regulation during vulva development in C. elegans
}

\author{
Dissertation \\ In partial fulfillment of the requirements for the degree \\ “Doctor of Philosophy” (Ph.D.) \\ Division of Mathematics and Natural Sciences \\ of the Georg-August-Universität Göttingen
}

\author{
submitted by \\ Yogesh Ostwal \\ Born in Ahmednagar, India
}

Göttingen, 2015 


\section{Members of the thesis committee}

Prof. Dr. Wolfgang Fischle ( $1^{\text {st }}$ reviewer $)$

Dept. of Chromatin Biochemistry,

Max-Planck Institute of Biophysical Chemistry,

Göttingen, Germany

and

Professor of Bioscience,

Biological and Environmental Sciences and Engineering,

King Abdullah University of Science and Technology,

Kingdom of Saudi Arabia

Prof. Dr. Herbert Jaeckle ( $2^{\text {nd }}$ reviewer $)$

Dept. of Molecular Developmental Biology, Max-Planck Institute of Biophysical Chemistry, Göttingen, Germany

\section{Prof. Dr. Michael Kessel}

Dept. of Developmental Biology,

Max-Planck Institute of Biophysical Chemistry,

Göttingen, Germany

\section{Prof. Dr. Dieter Klopfenstein}

Third Institute of Physics, Dept. of Biophysics, University of Göttingen,

Göttingen, Germany

Date of oral examination: $21^{\text {st }}$ October 2015 


\section{Affidavit}

I hereby declare that the presented thesis entitled "Regulation of binding of HP1 associated complexes to chromatin and their role in transcription regulation during vulva development in C. elegans" has been written independently and with no other sources and aids than quoted.

Göttingen, $10^{\text {th }}$ September 2015 


\section{Table of contents}

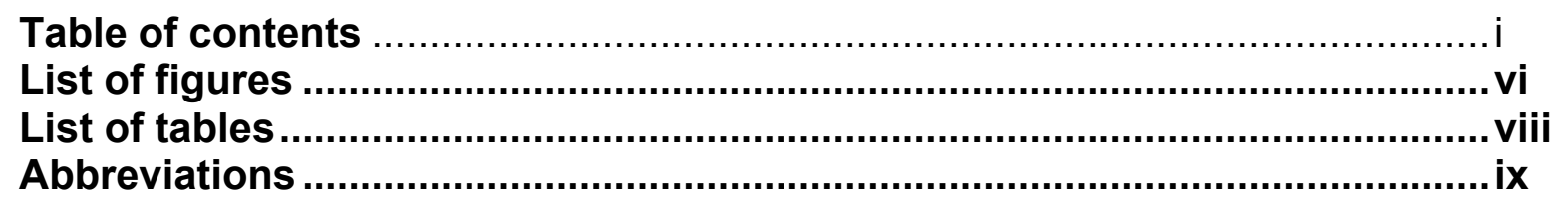

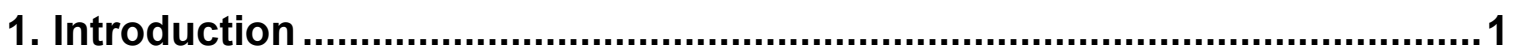

1.1 Chromatin organization in eukaryotes.................................................... 1

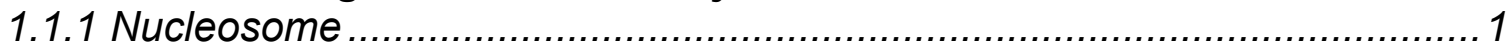

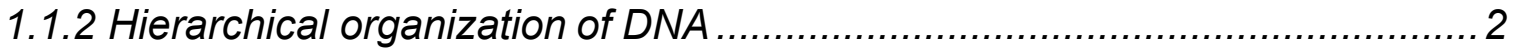

1.1.3 Euchromatin and heterochromatin ...................................................... 4

1.1.4 Epigenetics and chromatin states ........................................................ 4

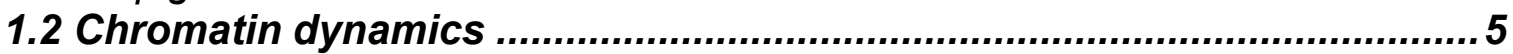

1.2.1 Regulation of opening and closing of chromatin .................................. 5

1.2.1.1 DNA methylation ........................................................................... 5

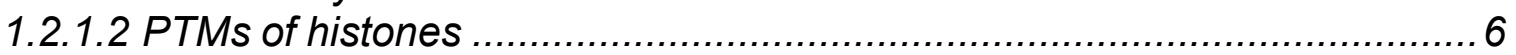

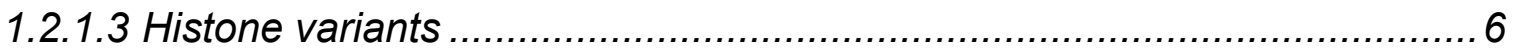

1.2.1.4 Chromatin remodeling complexes................................................. 7

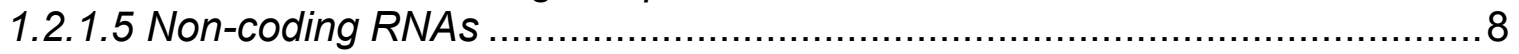

1.2.2 Systems analysis of chromatin states ........................................... 9

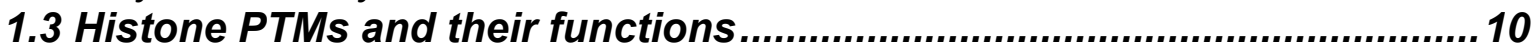

1.3.1 Histone PTMs affecting chromatin structure ........................................ 11

1.3.2 Histone PTMs recruiting effector proteins........................................... 13

1.4 Histone PTM readers ............................................................................... 13

1.4.1 Recognition modules ............................................................... 13

1.4.1.1 Specificity ............................................................................ 14

1.4.1.2 Regulation of PTM-reader interactions ........................................... 15

1.4.1.3 Functions of histone PTM-reader interactions .................................. 16

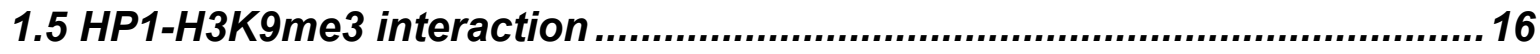

1.5.1 Heterochromatin protein 1 (HP1) ................................................... 16

1.5.2 CD-H3K9me3 interaction .................................................................. 17

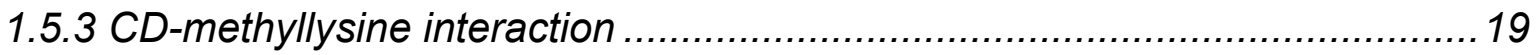

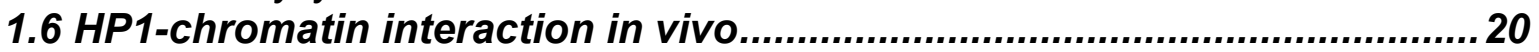

1.6.1 HP1-H3K9me3 interaction in heterochromatin function............................ 20

1.6.2 Factors affecting HP1-chromatin association ...................................... 21

1.6.2.1 Role of CSD and hinge region in HP1-chromatin association ...................21

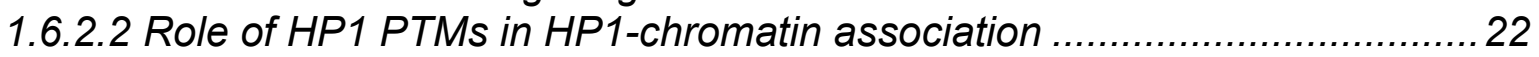

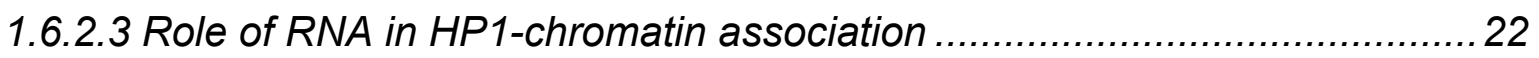

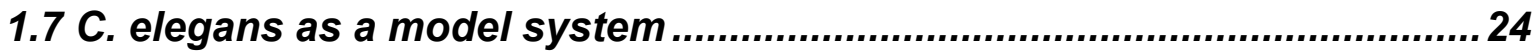

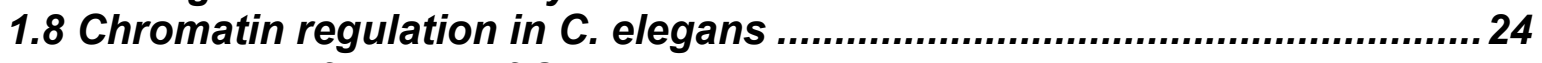

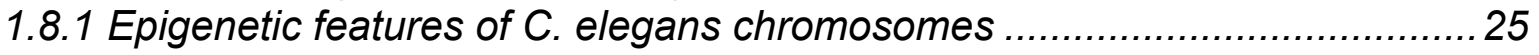

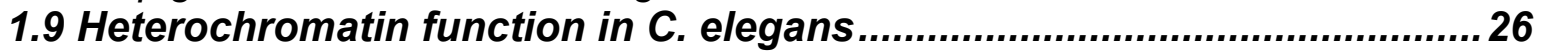

1.9.1 Role of HP1 proteins in heterochromatin function in C. elegans ..................27 
1.10 Mechanism(s) of HPL-2 recruitment to chromatin....................................2 27

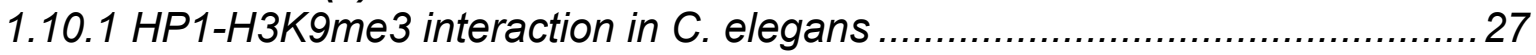

1.10.2 Role of CSD in recruitment of HPL-2 to chromatin ..................................27

1.10.2.1 LIN-13, a zinc finger protein ....................................................... 28

1.10.2.2 LIN-61, an MBT repeat protein ................................................... 28

1.10.3 Role of RNA in recruitment of HPL-2 to chromatin ..................................29

1.11 Vulva development and regulation in C. elegans ....................................29

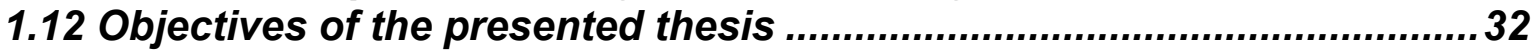

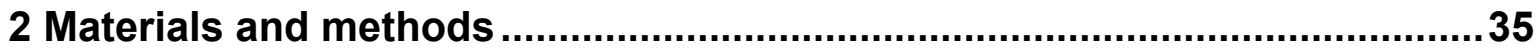

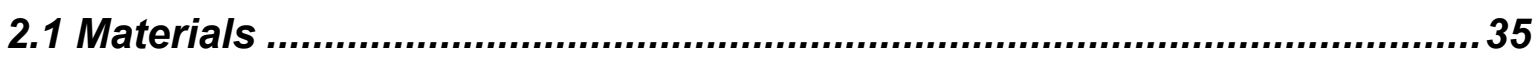

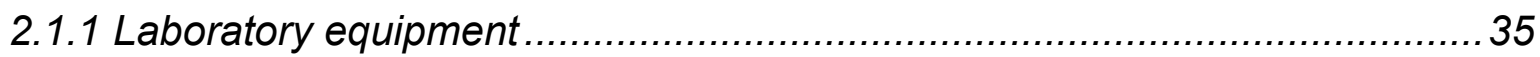

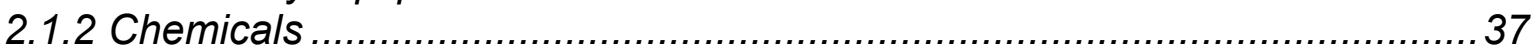

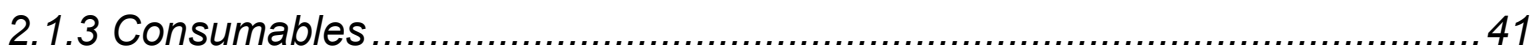

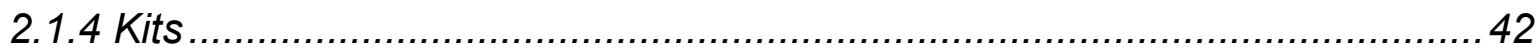

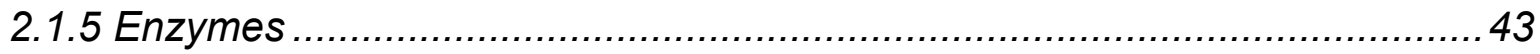

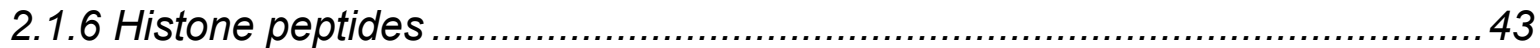

2.1.7 Antibodies obtained from different sources.......................................... 44

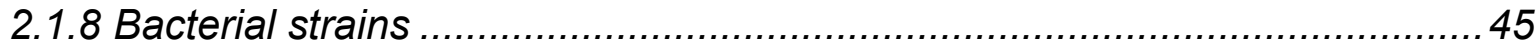

2.1.9 Plasmids used in this study........................................................ 46

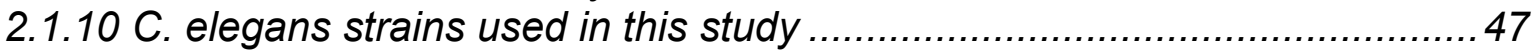

2.1.11 C. elegans strains generated in this study ....................................... 48

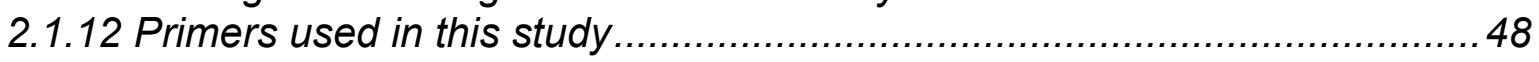

2.2 Molecular biological methods ...............................................................51

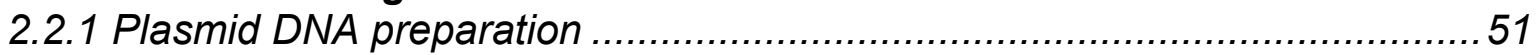

2.2.2 DNA digestion with restriction endonucleases....................................... 51

2.2.3 Polymerase chain reaction (PCR) ....................................................... 51

2.2.4 Agarose gel electrophoresis for separation of DNA fragments.................... 51

2.2.5 Transformation of plasmids into chemically competent bacteria ..................5 52

2.2.6 Preparation of chemically competent bacteria ........................................... 52

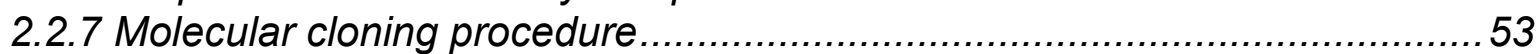

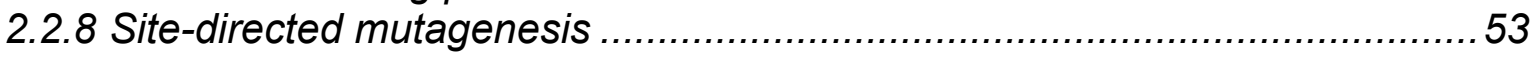

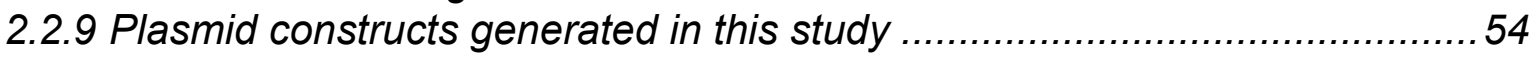

2.2.9.1 pGEX-4T3 based hpl-2 deletion constructs ..................................... 54

2.2.9.2 pGEX-4T3 based hpl-2 phosphomimic mutants ................................. 54

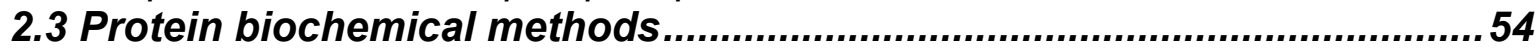

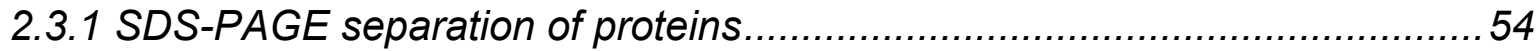

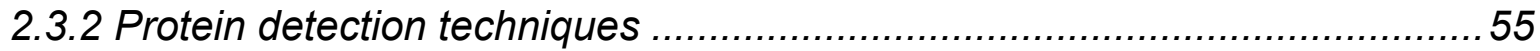

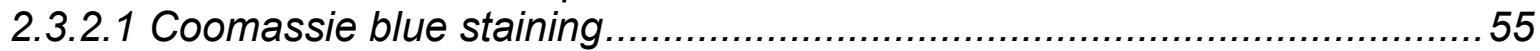

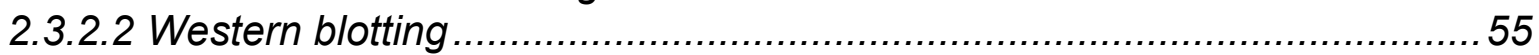

2.3.3 Expression of recombinant proteins in E. coli ....................................... 55

2.3.4 Purification of recombinant proteins ................................................... 56

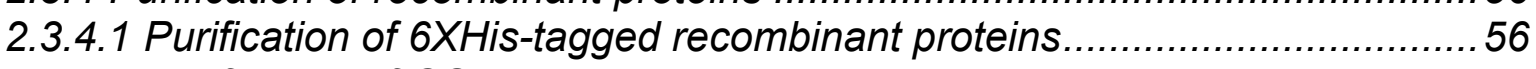

2.3.4.2 Purification of GST-tagged proteins............................................. 57 
2.3.5 In-vitro translation of Myc-tagged recombinant proteins using rabbit

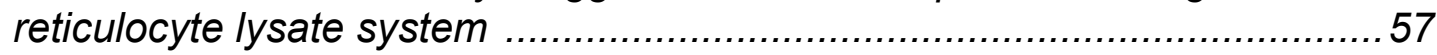

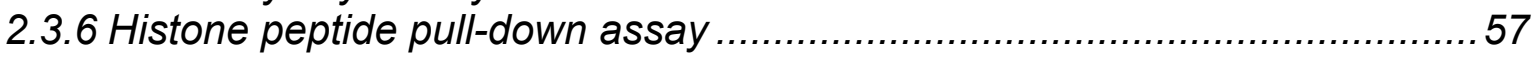

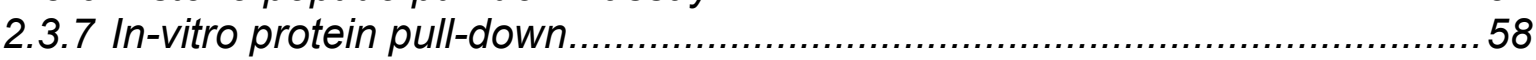

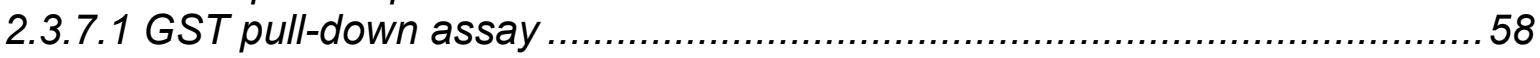

2.3.7.2 Ni-NTA pull-down assay ..................................................... 58

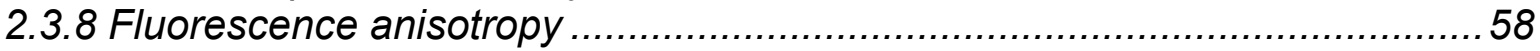

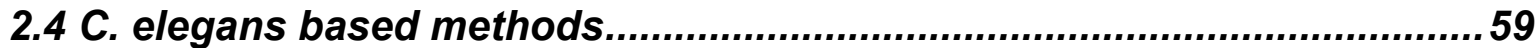

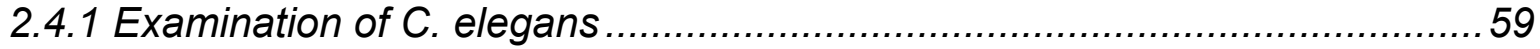

2.4.2 Culturing $C$. elegans on plates............................................................ 59

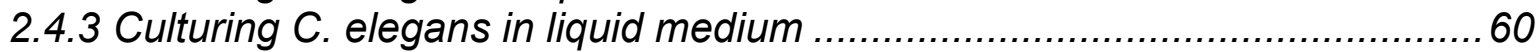

2.4.4 Bleaching of worms for synchronization or decontamination........................6 60

2.4.5 Freezing and recovery of $C$. elegans stocks..................................... 61

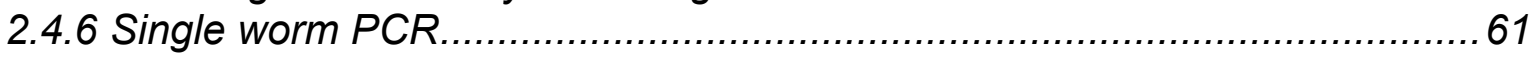

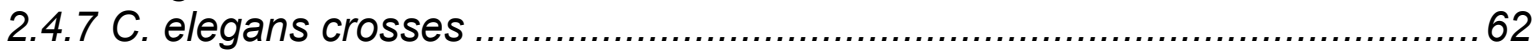

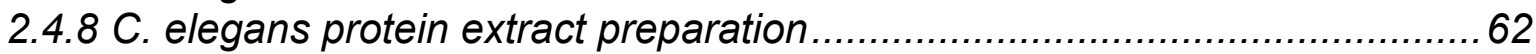

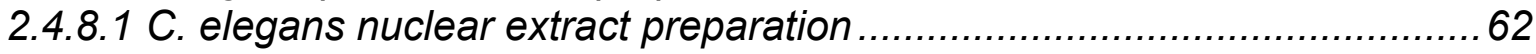

2.4.8.2 Generation of crude C. elegans lysate for western blot analysis...............63 63

2.4.9 Histone peptide pull-down assay using C. elegans extract.........................63 63

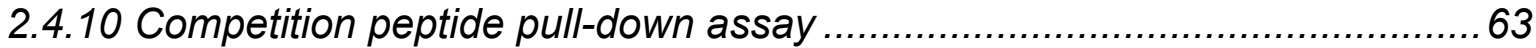

2.4.11 Immunoprecipitation from $C$. elegans extract .....................................63 63

2.4.11.1 Immunoprecipitation of endogenous proteins from $C$. elegans extract.... 63

2.4.11.2 GFP immunoprecipitation from C. elegans nuclear extract ....................64 64

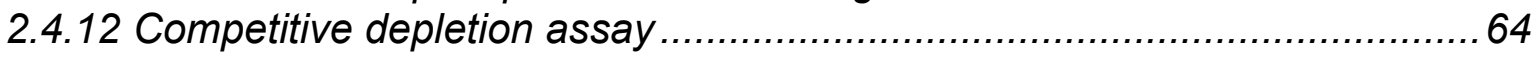

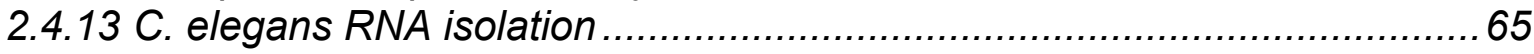

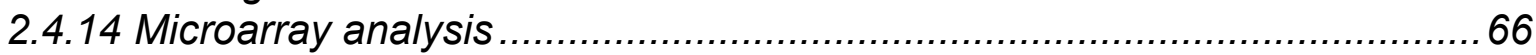

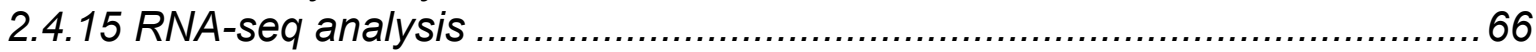

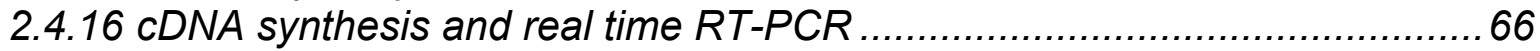

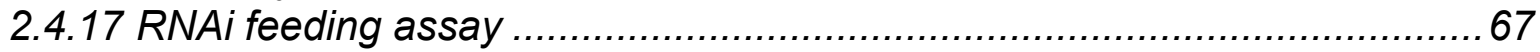

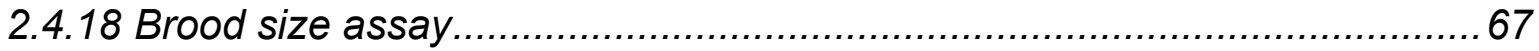

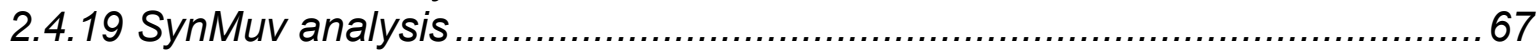

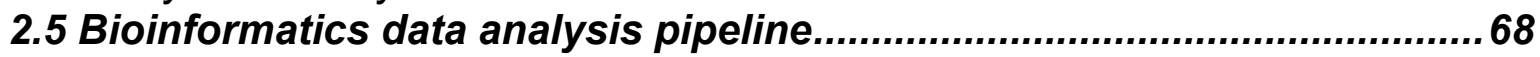

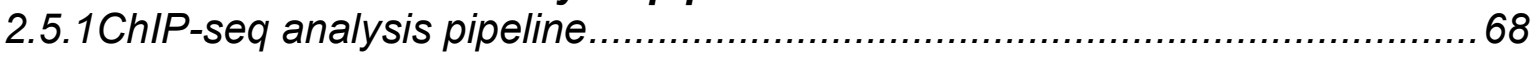

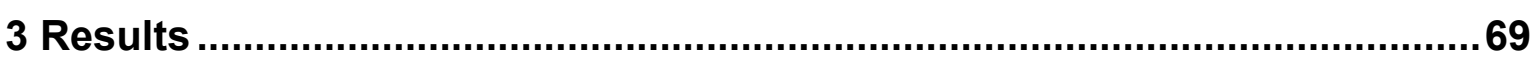

3.1 HPL-2-H3K9me3 interaction in C. elegans ..............................................69

3.1.1 HPL-2 associates with H3K9me3 only in the presence of LIN-61 ..............69

3.1.2 HPL-2 directly binds H3K9me3 in vitro .............................................. 70

3.1.3 HPL-2 binding to H3K9me3 is mediated by LIN-61 .............................. 73

3.2 HPL-2 and LIN-61 associate with each other .......................................... 76

3.2.1 HPL-2 and LIN-61 immunoprecipitate each other from C. elegans extract .. 76

3.2.2 HPL-2 and LIN-61 directly interact with each other in vitro......................... 76

3.2.3 HPL-2 interacts with LIN-61 via its CSD .......................................... 78

3.3 H3K9me3-LIN-61-HPL-2 signaling regulates vulva development via

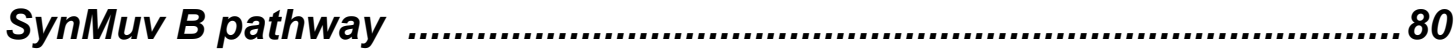


3.3.1 Characterization of various lin-61 alleles 80

3.3.2 H3K9me3-dependent LIN-61-HPL-2 pathway belongs to SynMuv B class of vulva development.

3.4 LIN-13 interacts with HPL-2 and LIN-61

3.4.1 HPL-2/LIN-61/LIN-13 co-immunoprecipitate each other.

3.4.2 Interaction between HPL-2 and LIN-13 is reduced in the absence of LIN61 while interaction between LIN-13 and LIN-61 is lost in the absence of HPL-2.

3.4.3 LIN-13 is H3K9me3 associated protein ............................................... 88

3.4.4 LIN-13 recovery on H3K9me3 is dependent on both LIN-61 and HPL-2..... 88

3.5 HPL-2/LIN-61/LIN-13 complex.

3.5.1 Full length HPL-2 is required for HPL-2/LIN-61/LIN-13 complex formation .. 90

3.5.2 HPL-2 forms multimers in vivo ........................................................... 92

3.6 lin-13 RNAi................................................................................................. 92

3.6.1 lin-13 RNAi in wild type N2 worms causes highly penetrant muv phenotype and sterility................................................................... 93

3.6.2 lin-13 RNAi in wild type N2 worms leads to decrease in HPL-2 and LIN-

61 protein levels but does not affect their mRNA levels ............................ 94

3.6.3 lin-13 genetically interacts with hpl-2 and lin-61 .................................. 96

3.7 HPL-2/LIN-61/LIN-13: functional genomics approach ............................98

3.7.1 Gene expression analysis of $\mathrm{hpl}-2$, lin-61 and lin-13 ............................. 98

3.7.1.1 Microarray analysis of $\mathrm{hpl}-2$ and lin-61 mutants ................................. 98

3.7.1.2 RNA-seq analysis upon lin-13 RNAi ........................................... 100

3.7.2 Identification and functional characterization of HPL-2/LIN-61/LIN-13

binding sites on C. elegans genome .................................................. 101

3.7.2.1 Identification of HPL-2/LIN-61/LIN-13 binding sites .............................. 101

3.7.2.2 Functional characterization of HPL-2/LIN-61/LIN-13 binding sites ............ 102

3.7.3 Functional characterization of distinct HPL-2/LIN-61/LIN-13 complexes..... 104

3.7.3.1 H3K9me3-dependent HPL-2/LIN-61/LIN-13 complexes........................... 104

3.7.3.2 H3K9me3-independent genome wide distribution of the

HPL-2/LIN 61/LIN-13 complexes ..................................................... 106

3.7.4 HPL-2/LIN-61/LIN-13 complexes regulate distinct subsets of target genes 107

3.7.4.1 Broad distribution of HPL-2-LIN-61 complex ........................................ 109

3.7.4.2 Sharp distribution of HPL-2-LIN-13 complex ...................................... 109

3.7.4.3 Bimodal distribution of HPL-2-LIN-61-LIN-13 complex ........................... 109

3.8 RNase-sensitivity of HPL-2/LIN-61/LIN-13 complexes............................... 111

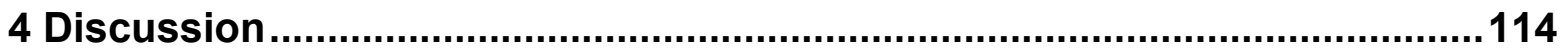

4.1 HP1-H3K9me3 interaction in C. elegans...............................................115

4.1.1 HPL-2 does not interact with H3K9me3 in vivo.......................................115

4.1.2 HPL-2 is a bonafide HP1 protein ......................................................... 115

4.1.2.1 HPL-2 might engage with non-histone methyllysine or in protein-protein interaction via its $C D$.................................................................... 117

4.1.2.2 Allosteric regulation of HPL-2-H3K9me3 interaction ..............................117

4.1.2.3 Possible PTMs of HPL-2 CD might negatively affect its affinity to H3K9me3 …........................................................................... 118

4.2 LIN-61 is a direct interaction partner of HPL-2.......................................118

4.2.1 Biological role of LIN-61 mediated HPL-2-H3K9me3 interaction .................119 
4.3 LIN-13, a zinc finger protein also binds HPL-2...................................... 120

4.3.1 HPL-2-LIN-61-LIN-13 complex ........................................................ 120

4.3.2 HPL-2 also forms distinct complexes with LIN-61 and LIN-13................... 121

4.4 LIN-13, acts in both SynMuv A as well as SynMuv B pathway of vulva development.

4.5 HPL-2/LIN-61/LIN-13 regulate transcription of genes associated with vulva development and fertility in C. elegans ......................................... 123

4.6 H3K9me3 and HPL-2 distribution does not correlate in vivo ..................... 124

4.7 Genome-wide distribution studies of HPL-2, LIN-61 and LIN-13 confirm the existence of multiple complexes among HPL-2, LIN-61 and LIN-13 .. 125

4.7.1 HPL-2-LIN-13 complex ................................................................... 125

4.7.2 HPL-2-LIN-61 complex ................................................................... 125

4.8 RNase sensitivity of HPL-2/LIN-61/LIN-13 complexes suggests the involvement of RNA component in the stability of these complexes .....126

4.9 Conclusion

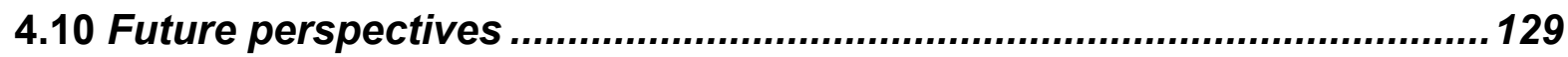

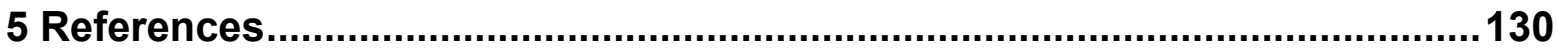

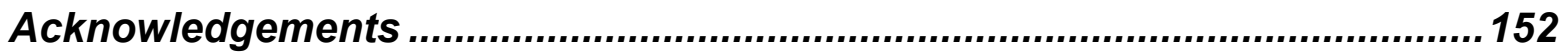

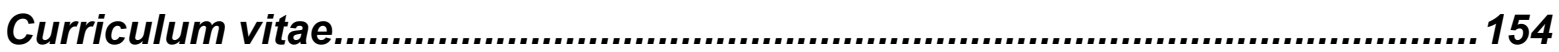




\section{List of figures}

Figure 1.1: Nucleosome core particle comprising 147 bp DNA wrapped around the histone octamer (adapted from (2))

Figure 1.2: Hierarchical organization of DNA in eukaryotes (adapted from (2)) .. 3

Figure 1.3: Regulation of opening and closing of chromatin ........................... 8

Figure 1.4: Hypothetical local chromatin states with unique epigenetic codes ... 10

Figure 1.5: Scheme showing domain organization of HP1 family proteins ......... 17

Figure 1.6: Structure of the HP1 $\beta$ CD-H3K9me3 complex (taken from (30)) ...... 19

Figure 1.7: Epigenetic features of $C$. elegans chromosomes........................... 26

Figure 1.8: Vulval induction signaling in $C$. elegans...................................... 31

Figure 3.1: Verification of HPL-2 as H3K9me3 binding protein ..........................69

Figure 3.2: HPL-2 binding to H3K9me3 is lost in lin-61 mutant extracts ............. 70

Figure 3.3: Recombinant HPL-2 binds H3K9me3 ...................................... 71

Figure 3.4: HPL-2 in C. elegans is a bonafide HP1 protein............................ 72

Figure 3.5: Recombinant HPL-2 binds H3K9me peptides............................ 73

Figure 3.6: HPL-2 binding to H3K9me3 is mediated by LIN-61 ..................... 74

Figure 3.7: Negative regulation of HPL-2-H3K9me3 interaction ...................... 75

Figure 3.8: HPL-2 and LIN-61 interact with each other in vivo ........................ 76

Figure 3.9: HPL-2 and LIN-61 directly interact with each other in vitro .............. 77

Figure 3.10: HPL-2 interacts with LIN-61 via its chromoshadow domain (CSD) in vitro.............................................................................. 79

Figure 3.11: LIN-61 and its interaction with $\mathrm{H} 3 \mathrm{~K} 9 \mathrm{me} 3$ is required for recovery of HPL-2 on H3K9me3 peptide ............................................ 82

Figure 3.12: HPL-2/LIN-61/LIN-13 co-immunoprecipitate each other ................. 85

Figure 3.13: Interaction between LIN-13 and LIN-61 is lost in the absence of HPL-2 while the interaction between LIN-13 and HPL-2 is reduced in the absence of LIN-61 .................................................. 87

Figure 3.14: LIN-13 is H3K9me3 associated protein.................................... 88

Figure 3.15: LIN-13 recovery to H3K9me3 is dependent on both HPL-2 and

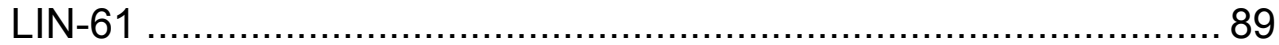

Figure 3.16: Recombinant CSD competes with endogenous HPL-2 for H3K9me3 binding and HPL-2 forms multimers in vivo ................. 91

Figure 3.17: lin-13 RNAi results in muv phenotype and sterility in C. elegans.... 93

Figure 3.18: lin-13 RNAi leads to decrease in HPL-2 and LIN-61 protein levels, but not mRNA levels .......................................................... 94

Figure 3.19: lin-13 RNAi does not affect LIN-61-H3K9me3 interaction ............... 95

Figure 3.20: lin-13 genetically interacts with lin-61................................ 96

Figure 3.21: Transcriptome analysis in lin-61, hpl-2 and lin-61; $h p l-2$ mutants ... 99

Figure 3.22: RNA-sequencing analysis upon lin-13 RNAi in C. elegans............ 100

Figure 3.23: Direct targets of HPL-2, LIN-61 and LIN-13 ............................ 103

Figure 3.24: ChIP-seq analysis of modENCODE datasets for H3K9me3, HPL-2,

LIN-61 and LIN-13............................................................. 105 
Figure 3.25: Statistically ranked models that could exist among HPL-2, LIN-61 and LIN-13 based on the abundance of their co-occupancy....

Figure 3.26: Direct targets of three distinct complexes of of HPL-2/LIN-61/LIN-13.108

Figure 3.27: Distinct distribution of HPL-2/LIN-61/LIN-13 complexes 110

Figure 3.28: HPL-2/LIN-61/LIN-13 complexes get disrupted upon RNase treatment

Figure 3.29: HPL-2/LIN-61/LIN-13 complexes get disrupted upon RNase treatment

Figure 4.1: Negative regulation of HPL-2-H3K9me3 interaction in C. elegans.

Figure 4.2: HPL-2/LIN-61/LIN-13 can form multiple complexes

Figure 4.3: Proposed model for recruitment of HPL-2/LIN-61/LIN-13 complexes ... 127 


\section{List of tables}

Table 1.1: Major histone PTMs and the enzymes that deposit and remove these modifications from chromatin (adapted from $(10,207)$ ) ..................... 12

Table 1.2: Histone PTMs and their recognition modules .................................. 14

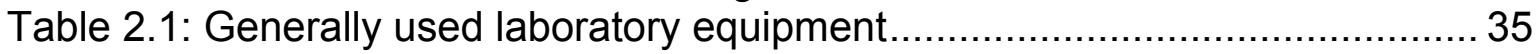

Table 2.2: Generally used chemicals ....................................................... 37

Table 2.3: Generally used consumables and other reagents ........................... 41

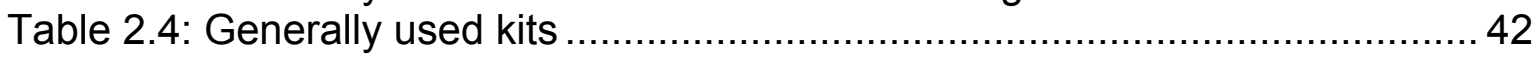

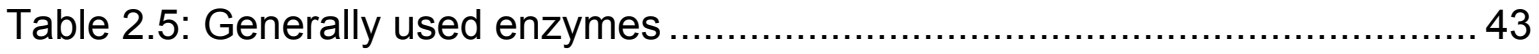

Table 2.6: Generally used biotinylated peptides ............................................. 43

Table 2.7: Generally used antibodies .......................................................... 44

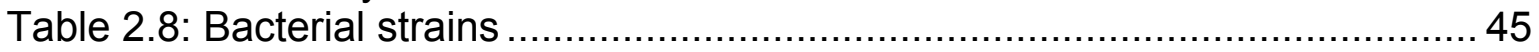

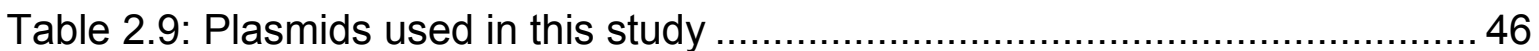

Table 2.10: $C$. elegans strains used in this study ....................................... 47

Table 2.11: C. elegans strains generated in this study.................................... 48

Table 2.12: Primers used in this study ..................................................... 48

Table 2.13: ChIP-seq datasets selected from modENCODE for analysis .............68

Table 3.1: Various LIN-61 mutant proteins and the strength of their interaction with H3K9me3

Table 3.2: SynMuv analyses of various lin-61 mutants ................................... 84 


\section{Abbreviations}

ac

AC

APS

ASH1

bp

BPTF

CARM1

CBX

CBP

C. elegans

CD

CENP-A

CHD

ChIP

ChIP-seq

CK2

$\mathrm{cm}$

CSD

DMP

DMSO

DNA

DNMT

dNTPs

DTT

E. coli

EDTA

GFP

GST

GCN5

G9a
Acetylation

Gonad anchor cell

Ammonium persulphate

Absent, small or homeotic-like protein

Base pairs

Bromodomain PHD finger transcription factor

Co-activator associated arginine methyltransferase 1

Chromo box

CREB-binding protein

Caenorhabditis elegans

Chromodomain

Centromere protein $\mathrm{A}$

Chromodomain helicase DNA binding protein

Chromatin immunoprecipitation

Chromatin immunoprecipitation-sequencing

Casein kinase 2

Centimeters

Chromoshadow domain

2,2-Dimethoxypropane

Dimethylsulfoxide

Deoxyribonucleic acid

DNA methyltransferase

Deoxyribonucleotide triphosphates

D,L-1,4-dithiothreitol

Escherichia coli

Ethylenediaminetetraacetic acid

Green fluorescent protein

Glutathione s-transferase

A class of histone acetyltransferase

A class of histone methyltransferase 
H3K9me1

H3K9me2

H3K9me3

H3K27me2a

H3R42me2a

H3K56ac

H3T118ph

H3K122ac

HAT

HDAC

HEPES

$\mathrm{His}$

HMGs

HOTAIR

HP1

HPL-2

HRP

hrs.

Hyp7

INO80

IP

IPTG

ISWI

$\mathrm{K}$

$\mathrm{K}_{\mathrm{D}}$

$\mathrm{kb}$

LB

LIN-61

linc RNA

LEM

MBD

MBT

MBTR
Histone H3 lysine 9 monomethylation

Histone H3 lysine 9 dimethylation

Histone $\mathrm{H} 3$ lysine 9 trimethylation

Histone H3 lysine 27 dimethylation

Histone $\mathrm{H} 3$ arginine 42 asymmetric dimethylation

Histone H3 lysine 56 acetylation

Histone H3 lysine 118 phosphorylation

Histone H3 lysine 122 acetylation

Histone acetyltransferase

Histone deacetylase

2-[4-(2-hydroxyethyl)-1-piperazinyl]-ethanesulfonic acid

Histidine tag

High mobility group proteins

HOX transcript antisense RNA

Heterochromatin protein 1

HP1-like-2

Horseradish peroxidase

Hours

Hypodermal syncytium hyp7

Inositol requiring mutant 80

Immunoprecipitation

Isopropylthio-b-D-galactoside

Imitation switch

Lysine

Dissociation constant

Kilobases

Lysogeny broth

Protein with four MBT domains in C. elegans

Long intergenic non-coding RNA

Lamina-associated polypeptide emerin-MAN1

Methyl binding domain

Malignant brain tumor domain

MBT repeat-containing protein 


\begin{tabular}{|c|c|}
\hline MDC1 & Mediator of DNA damage checkpoint 1 \\
\hline me & Methylation \\
\hline MeCP2 & Methyl CpG binding protein 2 \\
\hline MET & Methyltransferase (C. elegans) \\
\hline m & Meters \\
\hline$\mu g$ & Micrograms \\
\hline$\mu \mathrm{l}$ & Microliters \\
\hline$\mu \mathrm{M}$ & Micromolar \\
\hline $\mathrm{mg}$ & Milligrams \\
\hline $\min$. & Minutes \\
\hline $\mathrm{ml}$ & Milliliters \\
\hline $\mathrm{mm}$ & Millimeters \\
\hline $\mathrm{mM}$ & Millimolar \\
\hline MNase & Micrococcal nuclease \\
\hline modENCODE & Model organism encyclopedia of DNA elements \\
\hline muv & Multivulva phenotype \\
\hline ncRNA & non-coding RNA \\
\hline NGM & Nematode growth medium \\
\hline Ni-NTA & Nickel-loaded nitrilotriacetic acid coated agarose \\
\hline $\mathrm{ng}$ & Nanograms \\
\hline $\mathrm{nm}$ & Nanometers \\
\hline NRDE & Nuclear RNAi defective \\
\hline NuRD & Nucleosome remodeling and deacetylase complex \\
\hline NuRF & Nucleosome remodeling factor \\
\hline OD & Optical density \\
\hline $\mathrm{O} / \mathrm{N}$ & Overnight \\
\hline PARP1 & Poly-ADP-ribose polymerase \\
\hline PCAF & P300/CBP associated factor \\
\hline PRMT1 & Protein arginine methyltransferase- 1 \\
\hline PBS & Phosphate buffered saline \\
\hline PCR & Polymerase chain reaction \\
\hline PEG & Polyethyleneglycol \\
\hline $\mathrm{ph}$ & Phosphorylation \\
\hline
\end{tabular}




\begin{tabular}{|c|c|}
\hline PHD & Plant homeodomain \\
\hline PI5P & Phosphatidylinositol-5-phosphate \\
\hline PMSF & Phenylmethylsulfonyl fluoride \\
\hline PRC & Polycomb repressive complex \\
\hline PTM & Post-translational modifications \\
\hline $\mathrm{Rb}$ & Retinoblastoma protein \\
\hline RNA & Ribonucleic acid \\
\hline RNAi & RNA interference \\
\hline RNA Pol II & RNA polymerase II \\
\hline RNA-seq & RNA-sequencing \\
\hline rpm & Revolutions per minute \\
\hline RT & Room temperature \\
\hline SIRT1 & Sirtuin 1 \\
\hline sec. & Seconds \\
\hline SDS & Sodium dodecyl sulfate \\
\hline SDS-PAGE & Sodium dodecyl sulfate polyacrylamide gel electrophoresis \\
\hline SWI/SNF & Switch/sucrose non-fermentable \\
\hline SynMuv & Synthetic multivulva \\
\hline TBE & Tris borate EDTA \\
\hline TEMED & Tetramethylethylenediamine \\
\hline UHRF1 & Ubiquitin-like, containing PHD and RING finger 1 \\
\hline VPC & Vulval precursor cells \\
\hline WT & Wild type \\
\hline
\end{tabular}

Amino acid abbreviations are denoted by either first 3 letters of an amino acid or by one letter IUPAC code. Example: Tyrosine is referred to as either Tyr or Y. 


\section{Introduction}

\subsection{Chromatin organization in eukaryotes}

Deoxyribonucleic acid (DNA) in eukaryotes is spatially compartmentalized in a specialized organelle known as the 'nucleus'. The size of the nucleus ranges from 0.2-1 $\mu \mathrm{m}$ in general and does not fit the long DNA molecules. For example, human DNA is $3.3 \times 10^{9} \mathrm{bp}$ and if stretched would be $2 \mathrm{~m}$ long. Thus, DNA has to be packaged and organized in the nucleus in such a way that the information encoded in it can be retrieved as per the requirements of the cell. This packaging of DNA is mediated by an arsenal of proteins and RNA molecules, in a form called 'chromatin' (4). The basic unit of chromatin is known as the 'nucleosome'.

\subsubsection{Nucleosome}

In strict terms, the basic unit of chromatin consisting of a histone octamer with $147 \mathrm{bp}$ of DNA is known as 'nucleosome core particle' (figure 1.1) (1). There are four primary types of histones namely, H3, H4, H2A and H2B. Two copies each; of these four proteins form an octameric core around which the DNA is wrapped. The DNA makes 1.7 turns around the octameric core (197). Thus, chromatin appears like 'beads on a string' structure (figure 1.2) where these nucleosome core particles are linked via the 'linker DNA'. The fifth class of histone proteins, histone $\mathrm{H} 1$ binds to linker DNA. Nucleosome core particle along with histone $\mathrm{H} 1$ bound to linker DNA is known as 'nucleosome'. Histones are highly basic proteins, rich in arginine and lysine and thus possess intrinsic affinity to DNA (4). Positively charged histones interact with negatively charged posphodiester backbone of the DNA. 


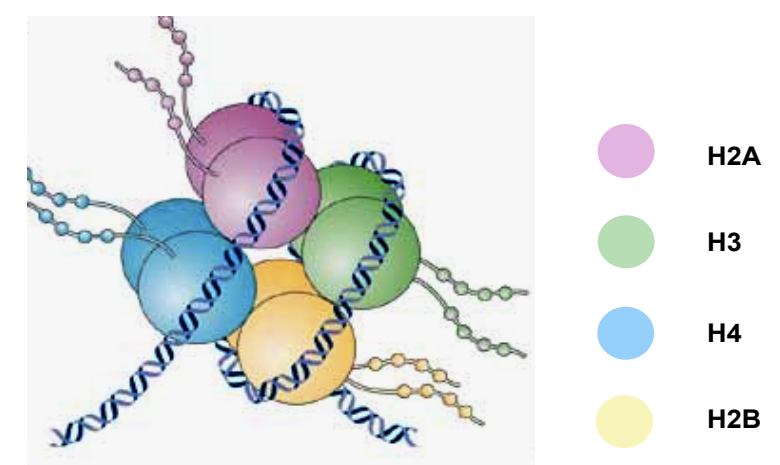

Figure 1.1: Nucleosome core particle comprising 147 bp DNA wrapped around the histone octamer (adapted from (2))

Histone proteins are highly conserved across all eukaryotic species from yeast to mammals (4). This suggests that the mechanisms of chromatin organization are also highly conserved across eukaryotic species.

\subsubsection{Hierarchical organization of DNA}

Chromatin organization largely depends on the cellular state such as stage of the cell cycle, cell type etc. Typically, the cell cycle consists of interphase and cell division phase. Interphase chromatin is less compact, loosely arranged whereas chromatin in the cell division phase undergoes higher order compaction to form structures called as 'chromosomes' (198).

In interphase, chromatin primarily exists in 'beads on a string' form although higher order forms of chromatin also exist in the cell (198). As shown in figure 1.2, this $11 \mathrm{~nm}$ fiber can be further packaged into a $30 \mathrm{~nm}$ form. Although, the in vivo existence of 30 $\mathrm{nm}$ fibers is questionable (201), electron microscopy analysis of isolated nucleosomal arrays have identified the $30-\mathrm{nm}$ structures upon increasing salt concentrations (200). 


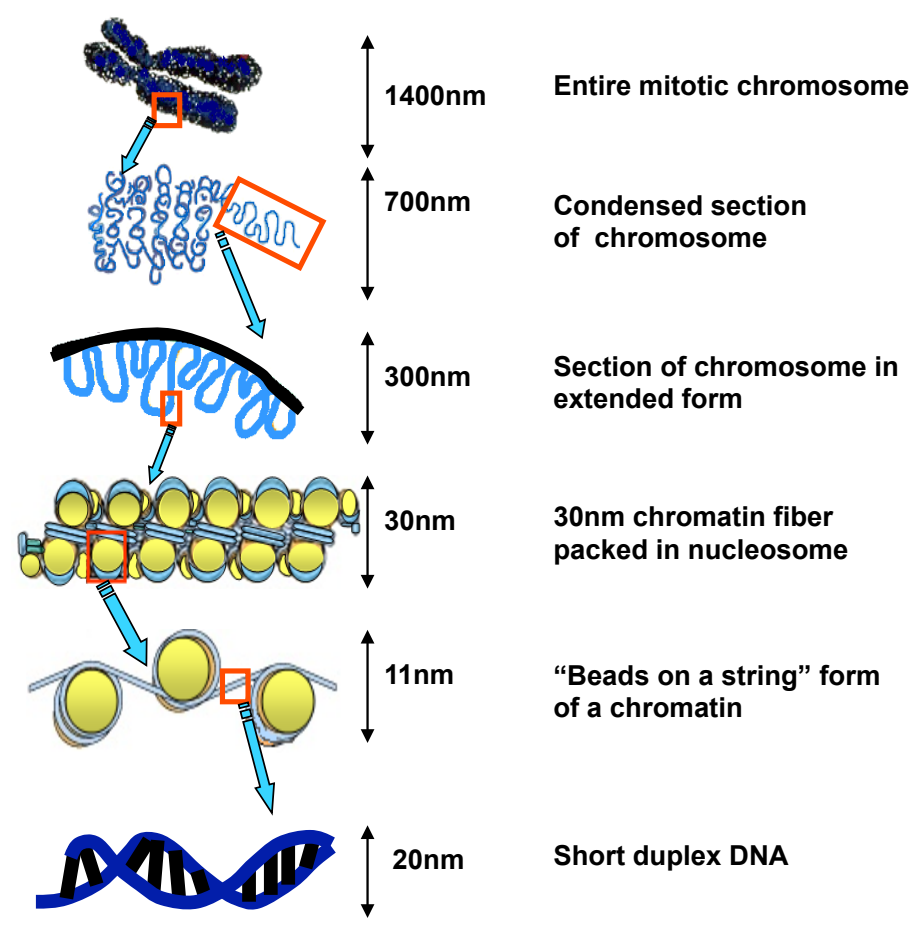

Figure 1.2: Hierarchical organization of DNA in eukaryotes (adapted from (2))

A naked DNA comprising double stranded DNA helix $(20 \mathrm{~nm})$ is wrapped around the histone octamer forming 'beads on a string' structure $(11 \mathrm{~nm})$. This $11 \mathrm{~nm}$ structure can be further folded to form $30 \mathrm{~nm}$ fibers. These $30 \mathrm{~nm}$ fibers can further form 'looped domains' giving rise to $300-700 \mathrm{~nm}$ highly condensed structures. These structures can ultimately fold into mitotic chromosomes $(198,199)$.

This implies that chromatin undergoes further compaction. This involves the packing of $30 \mathrm{~nm}$ fibers into a series of loops and coils to form 'looped domains' (198). These looped domains can be observed in special cell types such as lampbrush chromosomes of amphibian oocytes (199), which are otherwise extremely thin and small to be observed under the light microscope. These states can be condensed to 300-700 nm forms, although the molecular basis of these states is poorly understood. These forms can be further packaged into 'chromosomes', the ultimate unit of propagation of DNA from one generation to the next. 


\subsubsection{Euchromatin and heterochromatin}

Cytological studies have broadly defined two classes of interphase chromatin based on their state of condensation. A highly condensed form is referred to as 'heterochromatin' whereas the less condensed form is called 'euchromatin' (202). These two classes of chromatin have been further characterized based on their behavior in different experimental setups. Euchromatin is accessible to micrococcal nuclease (MNase), enzyme that can cleave naked DNA (158) and can be digested to $147 \mathrm{bp}$ reflecting the single nucleosomal conformation. It cannot be stained by 4', 6-diamidino-2-phenylindole (DAPI), a dye that stains DNA. The euchromatic DNA harbors actively transcribing genes, suggesting that it is transcriptionally active chromatin.

Heterochromatin on the other hand, consists of additional levels of compaction and thus, is not amenable to MNase (158). This is due to unavailability of naked DNA for nuclease digestion. Genomic regions that consist of high number of repetitive DNA, transposable elements found at centromeres, and telomeres, are present in heterochromatin form (203). These regions remain condensed throughout the development of an organism and also during different stages of the cell cycle and hence are known as 'constitutive heterochromatin' (203). On the other hand, many developmentally regulated gene loci, such as neural genes in non-neural tissues, undergo heterochromatin formation in a developmental signaling dependent manner and are referred to as 'facultative heterochromatin' (203). If an actively transcribing gene normally located in euchromatin, is experimentally translocated into heterochromatin region, it gets transcriptionally silenced. Such differences in gene expression are referred to as position effects, in which the expression of a gene depends on its location on a chromosome (204). Position effects were discovered in Drosophila and are commonly observed in several organisms (204). Position effects also exhibit an additional feature known as position effect variegation (PEV), in which patches of cells silence a gene that is juxtaposed to heterochromatin during development (204). These observations suggest that heterochromatin formation is developmentally regulated and once established can be inherited to daughter cells and is 'epigenetic' in nature.

\subsubsection{Epigenetics and chromatin states}

The term 'epigenetics' was coined by C. H. Waddington in 1942 to explain the 
differentiation of cells from a single-cell embryo (206). It refers to the phenomenon by which environmental or physiological non-genetic factors interact with genes to determine phenotype. Chromatin states, once established can be inherited upto several generations and hence are referred to as 'epigenetic'. Intensive studies in recent past has led to the identification of several components of chromatin that play important role in determining the epigenetic nature of these chromatin states and their dynamics (figure 1.2).

\subsection{Chromatin dynamics}

Chromatin dynamics refers to the reversible nature of chromatin states. Dynamic nature of chromatin allows efficient packaging of the DNA as well as provides access of the DNA to processes such as replication, transcription, repair and recombination.

\subsection{Regulation of opening and closing of chromatin}

Eukaryotic systems have evolved several mechanisms that allow efficient opening and closing of chromatin in a signaling dependent manner. These include DNA methylation, post-translational modifications (PTMs) of histones, incorporation of histone variants, recruitment of chromatin remodelers and non-coding RNAs $(3,4)$ (figure 1.3). All these mechanisms are described in brief below.

\subsubsection{DNA methylation}

DNA methylation involves addition of a methyl group to DNA. In mammals, DNA methylation mainly occurs at 5' position in cytosine in the context of $\mathrm{CpG}$ (159).

The mammalian genome contains stretches of $\mathrm{CpG}$ at a high frequency in promoters of many genes, and is referred to as 'CpG islands' (159). These are mainly hypomethylated as opposed to the $\mathrm{CpGs}$ present in non-CpG islands, which are mostly hyper-methylated (159). Methylation of CpG islands is strongly correlated with gene silencing (161). Interestingly, CpG islands are mostly found at the promoters of tissuespecific genes and Pol II transcribed genes (160). It has been suggested that DNA methylation is not necessary for initiation but is required for maintenance of transcription repression (159). DNA methylation is deposited and maintained by DNA 
methyltransferases (DNMTs). In mammals, DNMTs have been classified into 3 families viz. DNMT1, DNMT2 and DNMT3. While DNMT1 is a maintenance methyltransferase, DNMT3a and DNMT3b are de novo methyltransferases $(102,103)$. A weak methyltransferase activity has been demonstrated for DNMT2 (164). DNA demethylation on the other hand, can occur either actively or in a passive manner. Passive DNA demethylation occurs in the absence of DNA methylation activity after DNA replication, that leads to dilution of DNA methylation. Active DNA demethylation mechanisms have recently been described and they may occur via direct demethylation of 5-methylcytosine or via other intermediates $(165,166)$. Direct demethylation occurs via DNA base or nucleotide repair pathways while indirect DNA demethylation occurs via conversion of 5-methylcytosine to 5- hydroxymethylcytosine in enzymatic manner, which can then be repaired by base excision repair pathway (166). DNA methylation affects transcription via methylated $\mathrm{CpG}$ binding proteins that belong to the methyl binding domain (MBD) and kaiso families $(167,168)$. These proteins recognize methylated CpG on chromatin and recruit co-repressor complexes (167).

\subsubsection{PTMs of histones}

As described earlier, histones are small, highly basic proteins, which form the core of the nucleosome. These proteins are post-translationally modified at their $\mathrm{N}$ and $\mathrm{C}$ terminal tails as well as the globular domain (9). Histone PTMs either directly affect the association of histones to DNA or association of various nuclear proteins to DNA $(9,13)$. These are described in detail in section 1.3.

\subsubsection{Histone variants}

Apart from PTMs, non-allelic variants of histones with different sequences add to the complexity of chromatin regulation (169). Canonical histones are expressed only during s-phase of the cell cycle and are incorporated into DNA in a replication dependent fashion (170). On the other hand, histone variant genes are expressed throughout the cell cycle and are incorporated in a replication independent fashion by variant specific chaperones $(171,172)$. Several histone variants are restricted in their expression to certain tissues or are expressed during specific developmental stage(s). Due to 
differences in amino acid sequences, these histone variants, when incorporated into the nucleosomal core, induce structural changes that affect histone-histone interactions or histone-DNA interactions in the nucleosome. This in turn, can affect the stability of the nucleosomes as well as opening and closing of chromatin that can have functional consequences. For example, incorporation of a histone $\mathrm{H} 2 \mathrm{~A}$ variant, macroH2A increases nucleosomal stability and thus, promotes chromatin compaction and gene silencing (173). On the contrary, a particular histone $\mathrm{H} 3$ variant, $\mathrm{H} 3.3$ and another $\mathrm{H} 2 \mathrm{~A}$ variant, H2A.Z are associated with open chromatin conformation and transcriptional activation (174). Histone variants also have specialized functions. For example, the histone H3 variant, CENPA is specifically incorporated into centromeres, where it functions in the assembly of the kinetochore complex on the centromere (175). H2A.X, a histone variant of $\mathrm{H} 2 \mathrm{~A}$ is phosphorylated and recruited to DNA damage foci during DNA repair (176).

\subsubsection{Chromatin remodeling complexes}

Nucleosomes are intrinsic barriers to DNA-templated processes such as replication, transcription, repair and recombination (24). Thus, during these processes, nucleosomes need to be either mobilized or evicted in order to access the underlying DNA. This requires disruption of histone-DNA contacts in an ATP dependent manner. Cells possess specialized protein complexes known as 'chromatin remodeling complexes' that can perform either nucleosome sliding, nucleosome eviction or nucleosome spacing.

Various chromatin remodeling complexes can be divided into four classes based on the ATPase subunit and additional protein motifs (177) namely SWI/SNF family, ISWI family, CHD family and INO80 family. SWI/SNF family remodeling complexes bind to acetylated chromatin and are implicated in transcriptional activation (178). ISWI remodelers recognize nucleosomal features and thus, are responsible for nucleosome spacing and chromatin assembly (179). CHD family members are implicated in both transcriptional activation as well as repression based on the composition of their subunits. CHD complexes recognize H3K4 methylated chromatin and thus, promote transcription activation (180) whereas Mi-2/NuRD complex possess deacetylase activity and contains MBD proteins promoting transcription repression (180). 


\subsubsection{Non-coding RNAs}

Transcriptome analysis in many organisms has revealed the existence of a large number of non-coding RNAs (ncRNA) $(181,182)$. These can be classified into long or short ncRNAs. These ncRNAs have been implicated in chromatin structure and transcription regulation. For X-chromosome inactivation in mammals, Xist RNA is required (183). It possesses a unique stem loop structure known as 'repeat $A$ ' that recruits PRC2 to the $X$ chromosome to be inactivated (183). Another such ncRNA, HOTAIR binds to PRC2 via its 5' domain while its $3^{\prime}$ domain is involved in the association with LSD1 histone demethylase, thus allowing co-ordinate deposition of H3K27 methylation and H3K4 demethylation (184). One class of endogenous small RNAs known as short interfering RNAs (siRNAs) can directly inhibit transcription elongation by RNA pol II (185).

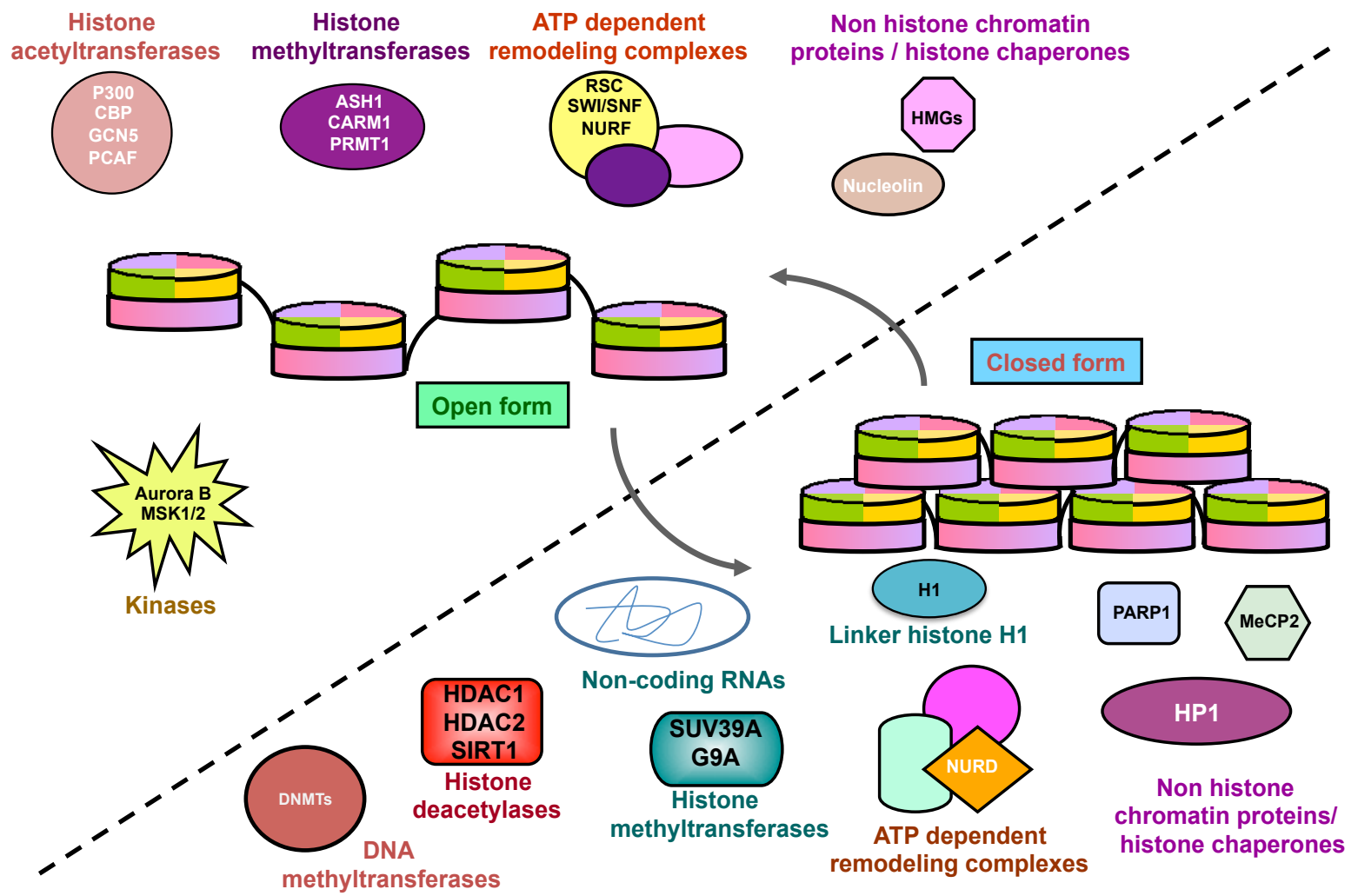

Figure 1.3: Regulation of opening and closing of chromatin

Various protein and RNA molecules regulate opening and closing of chromatin. Histone acetyltransferases (1.3), histone methyltransferases (1.3), ATP dependent remodeling 
complexes (1.2.1.4) and non-histone chromatin proteins facilitate formation of open chromatin structure. DNA methyltransferases (1.2.1.1), histone deacetylases (1.4), histone methyltransferases (1.4), ATP dependent chromatin remodeling complexes (1.2.1.4), non-histone chromatin proteins, linker histone $\mathrm{H} 1$, non-coding RNAs (1.2.1.5) facilitate formation of closed chromatin structure.

\subsection{Systems analysis of chromatin states}

Since, chromatin states are governed by several regulatory mechanisms involving chromatin modifications, protein and RNA molecules (1.2.1), it is important to understand the holistic outcome of these regulations that are exerted on chromatin.

Ever since the completion of the human genome sequence, efforts have been in place, to understand its organization and thus, function (186). A consortium known as 'Encyclopedia of DNA elements' (ENCODE) aims to understand the regulation of functional elements of the human genome (187). Similarly, the modENCODE consortium is designed to understand the genome organization in Drosophila and $C$. elegans (118). These consortia and other independent groups have performed computational studies integrating the genome wide distributions for several chromatin factors and modifications. These studies have revealed the existence of multiple chromatin states in flies and mammals $(5,6,7)$. Based on these studies, it can be deduced that each chromatin state is characterized by a unique molecular composition. The transcriptional state of a gene is determined by such local chromatin state(s), in which the gene resides. From a transcription perspective, local state of a chromatin can vary between two extreme states; tightly condensed, repressed chromatin state and actively transcribing chromatin state reflecting strong transcriptional repression and activation respectively (figure 1.4). Between these two states, there lies spectrum of chromatin states with varying degree of transcriptional potential. A local chromatin state can be found predominantly in one of such states. An ensemble of all such local chromatin states reflects global chromatin state of a genome, which in turn determines the transcriptome and corresponding proteome of a cell (6). In accordance to this hypothesis, a study in Drosophila cells has identified five principal types of chromatin based on the presence of unique composition of protein factors (7). These states can form domains of over $>100 \mathrm{~kb}$. Similar categorization has been carried out integrating 
genome wide distributions of several histone modifications in human cells (5).

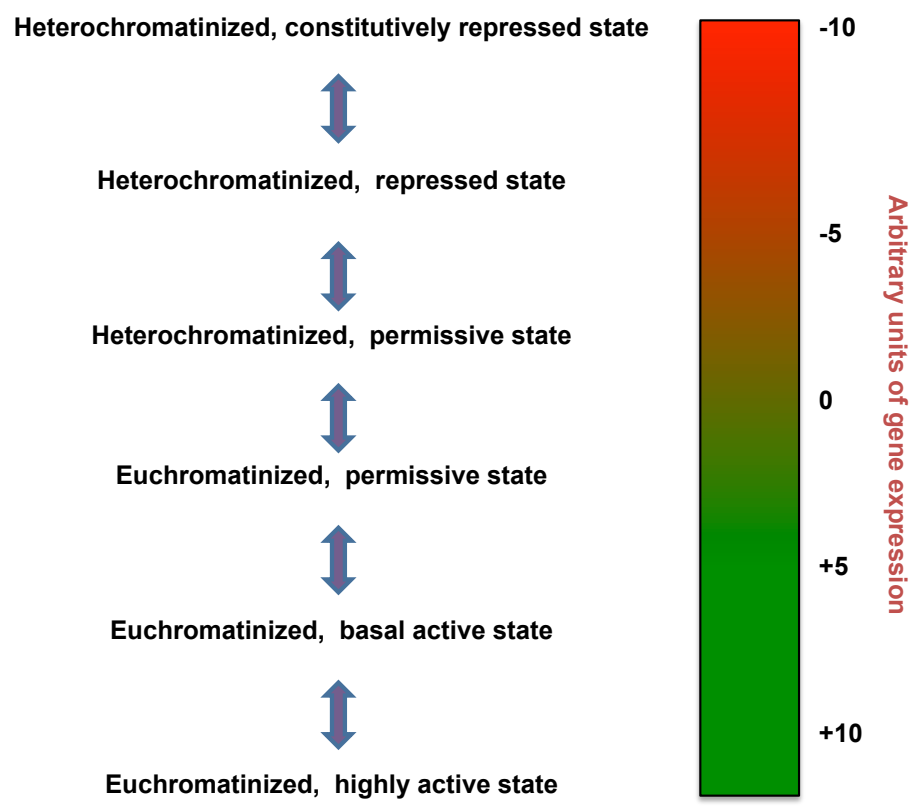

Figure 1.4: Hypothetical local chromatin states with unique epigenetic codes

A color plot indicates the degree of gene expression (red refers to silencing whereas green refers to activation) of the corresponding chromatin state. The schematic shows the existence of 6 chromatin states, from heterochromatinized, constitutively repressed state to euchromatinized, highly active state.

\subsection{Histone PTMs and their functions}

Histones are heavily subjected to post-translational modifications (9). Almost 200 different modifications of histones on 80 distinct sites have been reported so far and the list is still growing (3). Histone lysine acetylation, lysine and arginine methylation, serine, threonine and tyrosine phosphorylation, lysine ubiquitination are major known modifications across all eukaryotic species (193). Other minor histone modifications include sumoylation, ADP-ribosylation, proline isomerization, lysine propionylation and butyrylation as well as tyrosine hydroxylation (193). These modifications function mostly in a site-specific manner (3). The regulatory diversity is further increased by the degree of modification on a particular site. For example, histone $\mathrm{H} 3$ can be mono, di or tri methylated at lysine 4 and all three of these have different biological functions (9). The 
modifications are deposited and removed from chromatin in an enzymatic manner. Major histone modifications such as acetylation, methylation, phosphorylation and ubiquitination are listed in table 1.1 along with the enzymes that deposit and remove them from chromatin. These enzymes modify or remove specific sites on histones as well as non-histone proteins (10). Histone modifications modulate all the DNA-templated processes such as replication, transcription, repair and recombination. These modifications are thought to function in two different ways: either by directly affecting chromatin structure or by effector protein recruitment that recognize a particular modification.

\subsubsection{Histone PTMs affecting chromatin structure}

Histone modifications such as acetylation neutralize the basic charge of histone proteins thereby reducing their affinity to DNA (11). This leads to opening of chromatin and the underlying DNA is more accessible for processes such as transcription. Histone acetylation is therefore considered as positive regulator of gene expression whereas histone deacetylation is considered as positive regulator of gene silencing. Apart from charge neutralization, histone modifications in the core region can dramatically alter chromatin structure (188). Amino acid residues in histones that are closely situated to DNA entry-exit site in the nucleosome are also subjected to post-translational modifications and include H3K56ac and H3R42me2a. H3K56ac enhances DNA unwrapping at the entry-exit site (189) whereas H3R42me2a removes a potential hydrogen bond donor and decreases nucleosome stability and promote transcription in vitro (190). Modifications of histone residues located on the dyad axis also have an impact on nucleosome stability since histone-DNA interaction is strongest at the dyad axis (188). For example, H3K122ac leads to loss of water-mediated bridge between H3K122 and DNA (191) whereas H3T118ph drastically alters nucleosome structure (192).

Table 1.1: Major histone PTMs and the enzymes that deposit and remove these modifications from chromatin (adapted from $(10,207)$ ) 


\begin{tabular}{|c|c|c|c|}
\hline Modification & Position & $\begin{array}{l}\text { Enzymes that deposit } \\
\text { the modification }\end{array}$ & $\begin{array}{c}\text { Enzymes that remove the } \\
\text { modification }\end{array}$ \\
\hline \multirow{12}{*}{ Lysine Acetylation } & H3K9 & SRC1, PCAF & SIRT1, SIRT6 \\
\hline & H3K14 & $\begin{array}{l}\text { P300/CBP, TAF1, PCAF, } \\
\text { MOZ, MORF, TIP60 }\end{array}$ & \\
\hline & H3K18 & Р300/CBP & \\
\hline & H3K23 & P300/CBP & \\
\hline & H3K56 & Р300/CBP & SIRT1, SIRT2, SIRT6 \\
\hline & H4K5 & $\begin{array}{l}\text { HAT1, P300, TPI60, } \\
\text { HBO1 }\end{array}$ & \\
\hline & H4K8 & P300, TIP60, HBO1 & \\
\hline & H4K12 & P300, TIP60, HBO1 & \\
\hline & H4K16 & hMOF, ATF2, TIP60 & SIRT1, SIRT6 \\
\hline & H2AK5 & P300/CBP, TIP60, HAT1 & \\
\hline & H2BK12 & ATF2, P300/CBP & \\
\hline & H2BK15 & ATF2, P300/CBP & \\
\hline \multirow{6}{*}{ Lysine Methylation } & H3K4 & $\begin{array}{l}\text { MLL1-4, SET1, NSD2-3, } \\
\text { SET7-9, ASH1L, SMYD3 }\end{array}$ & LSD1, JARID1A-D, AOF1 \\
\hline & H3K9 & $\begin{array}{c}\text { SUV38H1/2, G9a, Eu- } \\
\text { HMT1, SETDB1, RIZ1, } \\
\text { ASH1 }\end{array}$ & $\begin{array}{l}\text { LSD1, JMJD2A-D, JHDM2A/B, } \\
\text { PHF8, KIAA1718 }\end{array}$ \\
\hline & H3K27 & EZH1/2, NSD2-3, G9a & \\
\hline & H3K36 & $\begin{array}{l}\text { SET2-3, NSD1-3, } \\
\text { SMYD2, ASH1L, } \\
\text { SETMAR }\end{array}$ & JMJD2A-C, JHDM1A/B \\
\hline & H3K79 & DOT1L & \\
\hline & H4K20 & $\begin{array}{l}\text { Pr-SET7, ASH1L, } \\
\text { NSD1-2, SUV4-20H }\end{array}$ & KIAA1718, PHF8 \\
\hline \multirow[t]{5}{*}{$\begin{array}{l}\text { Arginine } \\
\text { Methylation }\end{array}$} & $\begin{array}{l}\text { H3R2Me2a } \\
\text { H3R2Me2s }\end{array}$ & PRMT5-7 & JMJD6 \\
\hline & H3R8 & PRMT5 & PADI4 \\
\hline & H3R17 & PRMT4 & PADI4 \\
\hline & H3R26 & PRMT4 & \\
\hline & H4R3 & PRMT1/5 & JMJD6, PADI4 \\
\hline \multirow[t]{3}{*}{$\begin{array}{l}\text { Serine/Tyrosine } \\
\text { Phosphorylation }\end{array}$} & H3S10 & $\begin{array}{l}\text { MSK1/2, Aurora B, PIM1, } \\
\text { JNK }\end{array}$ & \\
\hline & H3S28 & MSK1/2, Aurora B & \\
\hline & H3Y41 & JAK2 & \\
\hline \multirow{2}{*}{$\begin{array}{l}\text { Lysine } \\
\text { Ubiquitinaton }\end{array}$} & H2AK119 & RING1B, 2A-HUB & USP3/16/21/22 \\
\hline & H2BK120 & $\begin{array}{c}\text { RNF20/40, UbcH6, } \\
\text { HR6A/B }\end{array}$ & USP3/22 \\
\hline
\end{tabular}

The nomenclature for histone modifications describes type of histone, initial letter of the amino acid residue that is modified, type of modification and degree of modification. For example, H3K9me2 describes histone $\mathrm{H} 3$ di-methylated at lysine 9. 


\subsubsection{Histone PTMs recruiting effector proteins}

There exist histone modifications such as methylation that do not directly change net charge of histones. These modifications act in more complex ways (12-14). Histone modifications create binding interfaces when deposited on a particular genomic domain. Specific protein domains recognize these binding interfaces and thereby associate with chromatin. These proteins then determine the functional outcome or serve as a platform for recruitment of other functional proteins. Such histone modification specific cascades could be coupled to regulate molecular processes. Several histone modifications act in this manner. The proteins that bind to chromatin in a histone modification dependent manner are called 'histone PTM readers'.

\subsection{Histone PTM readers}

Since the initial discovery of bromodomains as binders of H4K8ac (208), several structural and functional studies have led to the identification of 'histone PTM readers' $(15,16)$. These histone PTM readers contain structural folds/domains referred to as 'recognition modules' that recognize a particular histone modification and are present alone/in tandem/in combination with other domains in individual proteins, thereby increasing the complexity of the effective readout (15). This readout determines the functional consequences of histone modifications on a particular genomic domain.

\subsubsection{Recognition modules}

Recognition modules are the actual structural folds that are engaged with modified histones (15). These are structural modules that have specific size in order to allow modified histone residue to fit in. Based on the characteristics of the structural pocket, its size and the interactions with residues flanking modified amino acid, the specificity of recognition is determined (15). Table 1.2 summarizes the histone modifications and their respective 'recognition modules'. We describe some of the important features of the recognition modules.

Table 1.2: Histone PTMs and their recognition modules $(15,16)$ 


\begin{tabular}{|c|c|c|}
\hline Modification & Histone PTM site & Recognition module \\
\hline \multirow{7}{*}{ Lysine methylation } & H3K4me & Chromo, PHD, Tudor, MBT \\
\hline & H3K9me & Chromo, PHD, Tudor, Ankyrin repeats, WD40 \\
\hline & H3K27me & Chromo, WD40 \\
\hline & H3K36me & Chromo, PWWP \\
\hline & H3K79me & Tudor \\
\hline & H4K20me & Tudor, MBT, PWWP, WD40 \\
\hline & H1K26me & MBT, WD40 \\
\hline \multirow{2}{*}{ Arginine methylation } & H3R17me2a & Tudor \\
\hline & H4R3me2a & Tudor, ADD \\
\hline \multirow{2}{*}{ Serine phosphorylation } & H3S10 & 14-3-3, GCN5 \\
\hline & H2AXS139ph & BRCT \\
\hline \multirow[t]{3}{*}{ Lysine acetylation } & H3K14ac & Tandem PHD, Tandem bromo \\
\hline & H4K5ac,H4K8ac & Bromo \\
\hline & H4K16ac & Bromo \\
\hline Lysine ubiquitination & H2BK120 & CPS35 \\
\hline Lysine poly(ADP ribosylation) & $\mathbf{K}$ & Macrodomain \\
\hline
\end{tabular}

\subsubsection{Specificity}

The specificity of recognition of a histone PTM by a reader is determined by the structure of the recognition modules. For example, bromodomains possess a narrow but deep groove to accommodate only acetyl $\mathrm{K}$ and its long side chain (209). They interact only with acetyl $\mathrm{K}$, while neighboring residues are not involved in the interaction in a definitive manner (209). Thus, bromodomains can recognize any acetyl K irrespective of modification site in histones. Some of the bromodomains possess wider groove accommodating two acetyl $\mathrm{K}$ residues simultaneously, thus allowing simultaneous readout of two acetyl Ks in the same histone tail. For example, bromodomain in the reader protein BRDT interact with H4K5acK8ac accommodating two acetyl K residues (209).

Methylation readers on the other hand, are usually site-specific and sometimes can also discriminate between different states of methylation (15). These readers form an aromatic cage, in which methyl $\mathrm{K}$ fits in along with its long hydrophobic side chain (15). Mono and di-methyl $\mathrm{K}$ readers possess smaller pockets that cannot accommodate trimethyl $\mathrm{K}$ and thus provide specificity. On the other hand, tri-methyl $\mathrm{K}$ readers form deep 
and large aromatic pockets such that tri-methyl $\mathrm{K}$ fits in most stably. Apart from, aromatic cage-methyl $\mathrm{K}$ interaction, these readers also recognize neighboring amino acids, thereby recognizing methyl $K$ in a specific sequence context (15). This provides site-specificity to recognition. For example, the CD of heterochromatin protein 1 (HP1) recognizes $\mathrm{H} 3 \mathrm{~K} 9 \mathrm{me} 3$ specifically but not $\mathrm{H} 3 \mathrm{~K} 4 \mathrm{me} 3$ as the alanine at the -2 position fits into a small hydrophobic pocket and cannot be replaced by any other amino acid $(30,31)$. Some methylation readers do not make contacts with neighboring residues losing the sequence specificity. For example, MBT domain of L3MBTL1 recognizes all mono and di-methyl states irrespective of histone residue (142).

\subsubsection{Regulation of PTM-reader interactions}

Apart from the structural specificity that is conferred upon histone readers, the interaction between a histone PTM and its reader is regulated in several ways. The interaction of histone readers with other proteins, RNA and small molecules can regulate their interaction with a histone PTM in vivo. For example, PRC1 complex contains a protein CBX7 that is involved in the recognition of H3K27me2. Association of CBX7 with ncRNA is essential for it's binding to H3K27me2 (31). Another protein, UHRF1 possesses a tudor domain, which interacts with H3K9me3 only in the presence of an allosteric activator, phosphatidylinositol phosphate, PI5P(210).

Post-translational modifications of readers can disrupt the histone PTM-reader interactions. For example, acetylation of the RSC4 subunit of the chromatin remodeler, RSC can inhibit its bromodomain-mediated interaction with H4K14ac (16). Histone PTMs in the neighboring residues also have major effects on reader-PTM interactions. H3S10ph inhibits HP1 binding to H3K9me3 (56). On the other hand H3K14ac enhances 14-3-3 binding to H3S10ph (211).

\subsubsection{Functions of histone PTM-reader interactions}

The functions of histone PTM recognition by reader proteins largely depend on the characteristics of these proteins and their interaction partners. For example, as a part of a chromatin-remodeling complex, BPTF reads $\mathrm{H} 3 \mathrm{~K} 4 \mathrm{me}$ and acetyl $\mathrm{K}$. This recruits the NURF complex to these regions to facilitate transcriptional activation (212). These 
readers are also part of modification cascades recruiting other histone modifiers. The RPD3S histone deacetylase complex recognizes H3K36me via its chromodomain and deacetylates the nucleosomes, thereby preventing cryptic transcription initiation (213). Readers are also part of specialized pathways such as DNA repair. Near DNA damage sites, H2AX139ph is read by MDC1, which then recruits DNA repair enzymes (16). HP1 proteins recognize H3K9me3 and thereby localize to heterochromatic regions. We focus on this interaction and its role in heterochromatin formation in the next section.

\subsection{HP1-H3K9me3 interaction}

\subsubsection{Heterochromatin protein 1(HP1)}

HP1 is a major determinant of heterochromatin discovered in Drosophila as a suppressor of PEV phenotype $(26,27)$. Importantly, this protein is known to bind to H3K9me3 via its chromodomain (CD) and thereby, localizes to pericentric heterochromatin (29). It also has a chromoshadow domain (CSD) required for the dimerization of the protein as well as protein-protein interactions (194) and an unstructured hinge region that connects both the domains (figure 1.5).

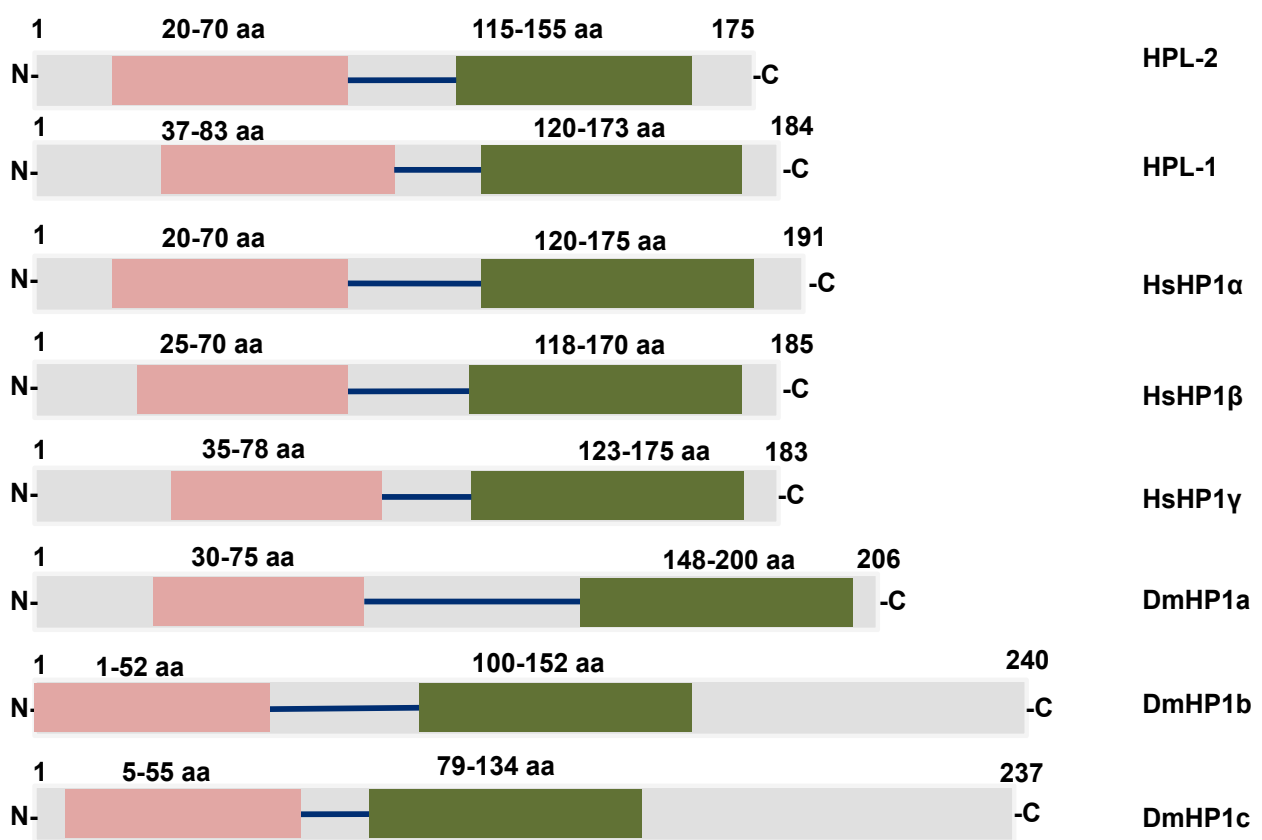




\section{Figure 1.5: Scheme showing domain organization of HP1 family proteins}

Domain organization of HP1 proteins in C. elegans (HPL-1 and HPL-2), H. sapiens

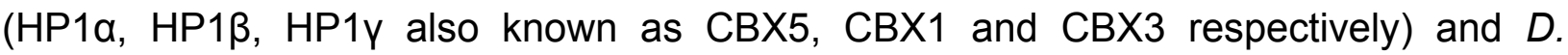
melanogaster (HP1a, HP1b and HPc) is shown.

\subsubsection{CD-H3K9me3 interaction}

In the HP1 CD-H3K9me3 peptide complex, peptide remains in a $\beta$-sheet like conformation while CD adopts a fold. The N-terminus of CD wraps around the peptide bringing Tyr21, Trp42 and Phe45 closer to the peptide (30). The methyl group fits into the pocket formed by mutually orthogonal aromatic side chains of these 3 conserved amino acids (figure 1.6). In the absence of methyl groups, the rigid side chains of these aromatic amino acids cannot form a tight cluster and fall apart. Thr51 of CD binds to methyllysine side chain of the peptide whereas Glu53 and Asp57 interact with peptide backbone at the other end of methyllysine (30). These two sets of interactions define methylated Lys9 site and are also conserved among all HP1 chromodomain proteins.

The HP1 CD does not bind to free methyllysine. The context in which the methyllysine is presented is responsible for binding because CD makes additional contacts with the peptide. The four amino acids that precede Lys9 of the H3 tail, QTAR interact with the CD. The Alanine residue at -2 position from methyllysine is crucial for CD-peptide interaction. Alanine side chain fits exactly in the hydrophobic pocket formed by Val23, Leu40, Trp42, Leu48 and Cys60 (30). Replacement of alanine with an amino acid containing a bulky side chain leads to disruption of this interaction. This provides partial specificity to sequence contexts in which methyllysine is presented with alanine at -2 position. H3K4me and H4K20me sequence contexts (possess arginine at -2 position) do not meet this requirement and hence do not bind CD of HP1.

Glutamine at -4 position interacts with N-terminal $\alpha$-helix of CD. In H3K27me context, the presence of Lys at -4 position, which is positively charged and bulky, makes the binding much weaker. Threonine at -3 position is an important determinant for selective binding of H3K9me3 at least for HP1y and HP1a. This Threonine is sandwiched in a negatively charged polar fingers/clasp formed by Glu29/19 and Asp68/58 of HP1Y and HP1 $\alpha$ respectively (31). 
In the context of nucleosomes however, biochemical studies have shown that only CD might not be sufficient for the HP1-nucleosome interactions $(214,134)$. This is also supported by the fact that hinge region and CSD also contribute to heterochromatic localization of HP1 (123) and might make additional contacts with nucleosomes. However, NMR study on HP1 1 -H3K9me3 nucleosomes indicates that CD is the only region that stably interacts with $\mathrm{H} 3 \mathrm{~K} 9 \mathrm{me} 3$ in a highly mobile complex (32). N-terminal tail and hinge region make weak contacts with nucleosomes. It was further suggested that $\mathrm{CD}-\mathrm{H} 3 \mathrm{~K} 9 \mathrm{me} 3$ interaction in the context of nucleosomes is structurally equivalent to that in the context of an H3-peptide. Moreover, the binding mode of HP1 to unmodified nucleosomes does not involve $C D$ but rather hinge region while $\mathrm{N}$-terminal tail transiently interacts with nucleosomal DNA (32). 

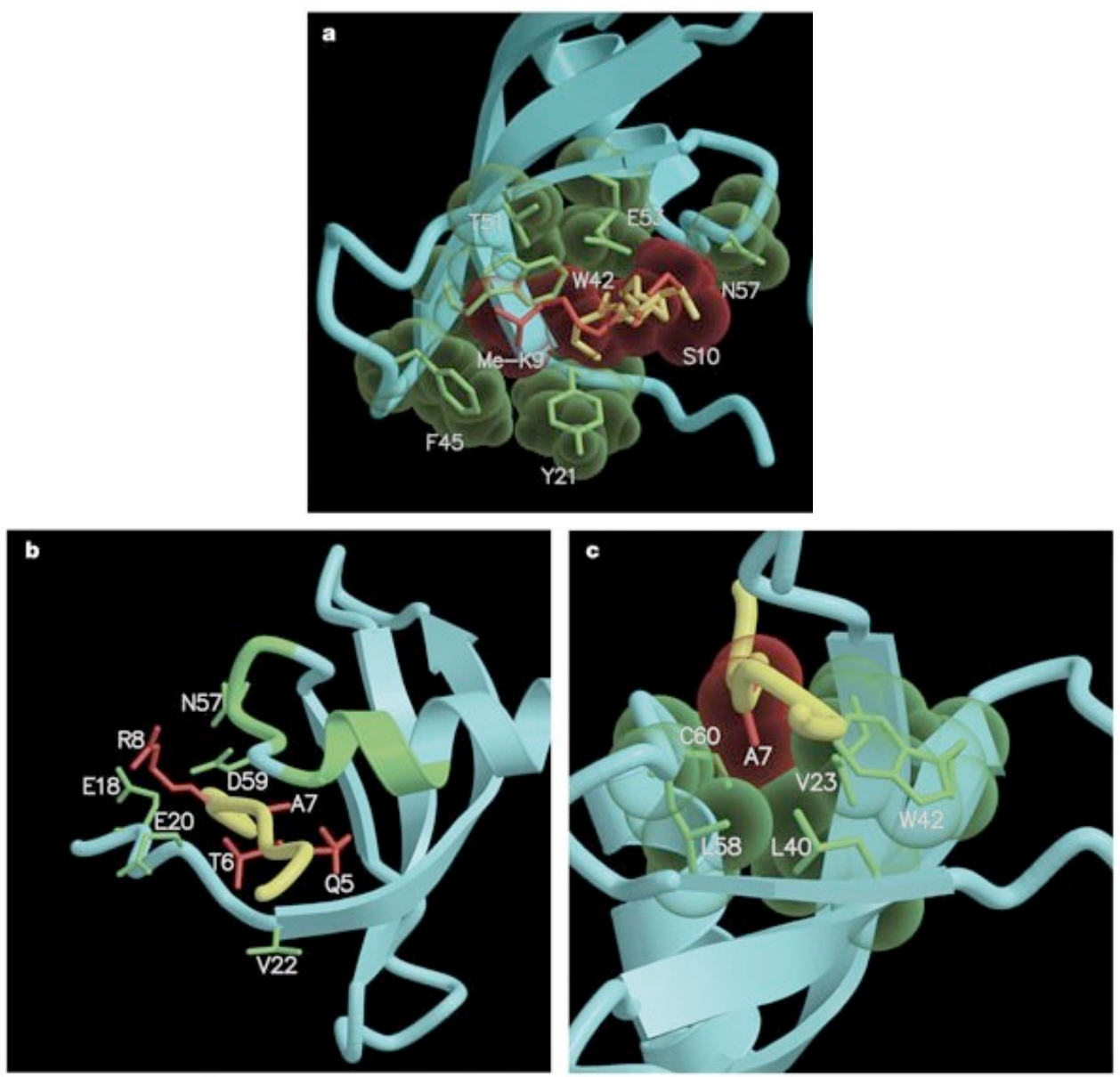

Figure 1.6: Structure of the HP1 $\beta$ CD-H3K9me3 complex (taken from (30))

Side chains in the H3K9me3 peptide are highlighted in red while interacting residues in the CD are highlighted in green. (a) Tyr21, Trp42 and Phe45 form a hydrophobic pocket in which $\mathrm{H} 3 \mathrm{~K} 9 \mathrm{me} 3$ fits in. (b) Gln5 interacts with the N-terminus of $\alpha$-helix while Arg8 interacts with the acidic patch. (c) Ala7 binds in the core of the CD, and makes contact with residues Val 23, Leu 40, Trp 42, Leu 58 and Cys 60.

\subsubsection{CD-methyllysine interaction}

Apart from $\mathrm{H} 3 \mathrm{~K} 9 \mathrm{me} 3$, the $\mathrm{CD}$ of $\mathrm{HP} 1$ proteins also recognize methyllysine residues in histone $\mathrm{H} 1$ and non-histone proteins (35). The CD of HP1y binds H1K26me (36), G9aK185me (37), pRBK873me (195). Structurally, compared to H3K9me3 peptide, H1K26me and G9aK185me peptides do not form an extended $\beta$-strand although CD adopts a canonical fold (38). The amino acids KKAR(K26)S of Histone $\mathrm{H} 1$ and HRAR(K185)T are buried in the groove of CD of the HP1y, where Val32 of HP1y form 
hydrogen bonds with H1K22 and G9aH181. Again, alanine at -2 position has a side chain that is buried in a hydrophobic pocket in the same way as HP1 $\beta-H 3 K 9 m e 3$ peptide complex. This is a requisite for HP1-methyllysine interaction.

\subsection{HP1-chromatin interaction in vivo}

Since, HP1 proteins associate with H3K9me3 in vitro, it is expected that these proteins get recruited to chromatin in an $\mathrm{H} 3 \mathrm{~K} 9$ me3 dependent manner. H3K9me3 is a hallmark of heterochromatin and is associated with transcriptionally silent regions (203). On the other hand, HP1 proteins were discovered in Drosophila as suppressors of variegation and are implicated in heterochromatin function $(26,27)$. Thus, for a long time, it was thought that HP1 proteins bind $\mathrm{H} 3 \mathrm{~K} 9 \mathrm{me} 3$ in cells and bring about heterochromatin function.

\subsubsection{HP1-H3K9me3 interaction in heterochromatin function}

Studies from several model systems have contributed to the understanding of role of HP1-H3K9me3 interaction in heterochromatin function (203). Removal of enzymes catalyzing $\mathrm{H} 3 \mathrm{~K} 9 \mathrm{me} 3$ and the effect of loss of $\mathrm{H} 3 \mathrm{~K} 9 \mathrm{me} 3$ on the recruitment of HP1 proteins was one of the earliest approaches undertaken to study HP1-H3K9me3 interaction. In fission yeast S. pombe, clr4 and swi6 genes encode for homologues of SUV39H (H3K9me3 catalyzing enzyme) and HP1 respectively. Disruption of enzymatic activity of CLR4 results in loss of $\mathrm{H} 3 \mathrm{~K} 9 \mathrm{me} 3$ from pericentric heterochromatin with concomitant loss of SWI6 protein from heterochromatin (155). This leads to defects in chromosomal segregation (155). Both these proteins are essential for heterochromatic gene silencing and centromere function (155). In SUV39H null mutants in flies, HP1a enrichment to heterochromatin is reduced while its association to euchromatin is unaltered (50). Moreover, HP1a null mutant is lethal whereas SUV39H mutants are viable in flies, suggesting that HP1a mainly functions outside H3K9me3 pathway. In mammals, of the three HP1 isoforms, HP1 $\alpha$ and HP1 $\beta$ are primarily localized to heterochromatin while $\mathrm{HP} 1 \mathrm{y}$ localizes to euchromatin suggesting functional specialization of these isoforms (123). However, in vitro, all three isoforms bind to H3K9me3. Studies involving abrogation of SUV39H1 and SUV39H2 in mice show 
partial delocalization of HP1 proteins from heterochromatic regions (42). All these studies suggest that HP1 proteins associate with chromatin in H3K9me3 dependent as well as H3K9me3 independent manner.

\subsubsection{Factors affecting HP1-chromatin association}

Several factors such as protein-protein interactions via CSD or hinge region, PTMs, RNA molecules etc., can regulate association of HP1 with chromatin in vivo.

\subsection{Role of CSD and hinge region in HP1-chromatin association}

It was discovered that the isolated CD of HP1 when expressed in mammalian cells alone cannot localize to pericentric heterochromatin suggesting the involvement of CSD and hinge region in the localization of HP1 proteins to heterochromatin $(134,214)$. Since, CSD is capable of multimerization in vitro, it has been proposed that CSD is involved in dimerization of HP1 proteins and thereby facilitates spreading of HP1 domains in pericentric heterochromatin (123). It has further been proposed that HP1 via its CSD interacts with SUV39H and in turn, promotes H3K9me3 deposition which might account for its spreading along heterochromatin (123).

In contrast to this, recent studies in yeast have shown that recognition of H3K9me3 by SWI6 relies on the interface between two CDs (33). This causes SWI6 to tetramerize generating two sticky end CDs. Subsequently; authors (34) have identified two different states of SWI6 protein that influence its H3K9me3 binding. In an auto-inhibited state, a histone mimic sequence (ARK loop) in one SWI6 monomer prevents the recognition of H3K9me3 by CD of another monomer. In the context of H3K9me3 and nucleosomal DNA, this state is unlocked leading to a spreading competent state where CD-CD interaction leads to H3K9me3 binding and two unbound sticky CDs are exposed (33, 34). However, this mechanism is exclusive to yeast SWI6 owing to its long N-terminal region, which is absent in other HP1 homologues, and thus other HP1 proteins may not behave in this manner. 


\subsubsection{Role of HP1 PTMs in HP1-chromatin association}

HP1 proteins are subjected to extensive post-translational modifications in vivo (130). In fact, it has been suggested that HP1 possesses histone-like subcode (133). These PTMs can affect their ability to interact with $\mathrm{H} 3 \mathrm{~K} 9 \mathrm{me}$. Recently, it has been shown that $\mathrm{N}$-terminal phosphorylation of HP1a increases its affinity to H3K9me3 (126). One study has shown that phosphorylation site mutations (both, serine-to-alanine and serine-toglutamine) in HP1a reduce or eliminate its silencing ability in Drosophila (131).

Phosphorylation of HP1 $\beta$ at Thr51 by CK2 leads to its mobilization from chromatin (132). The hydroxyl moiety of Thr51 is involved in hydrogen bond network that holds $\mathrm{HP} 1 \beta$ around $\mathrm{H} 3 \mathrm{~K} 9 \mathrm{me} 3$. The disruption by either phosphorylation or a mutation results in loss of $\mathrm{H} 3 \mathrm{~K} 9 \mathrm{me} 3$ binding of HP1 $\beta$ resulting in its mobilization from chromatin in vivo (132). Similarly, HP1y is phosphorylated by Protein kinase A at Ser83 (133). This phosphorylated pool of HP1y has an exclusively euchromatic localization (133). This study also reported that either phosphorylation or mutation of Ser83 results in loss of its binding to $\mathrm{H} 3 \mathrm{~K} 9 \mathrm{me} 3$. Thus, phosphorylation can have either positive or negative effect on HP1-H3K9me3 interaction.

\subsection{Role of RNA in HP1-chromatin association}

Although, CDs were identified as one of the earliest RNA-binding modules (59), HP1 CDs lack the ability to bind RNA (60). HP1 1 protein possesses a hinge region with clusters of positively charged amino acids, which interact with RNA in vitro. (61-63). Studies have also shown that HP1 localization to pericentric heterochromatin is RNase A sensitive in mouse cells (61). Moreover, addition of RNA, back from the same cellular source but not tRNA or bacterial RNA restores the heterochromatic localization (64). HP1 1 has been shown to directly interact with major satellite repeat RNA forward transcripts (63). The same study also showed that this interaction is essential for HP1a targeting to pericentric heterochromatin (63). Similarly, for HP1y, RNA mediated recruitment to specific genomic loci in human cells has been proposed (65). HP1 $\beta$ and HP1y have been also identified as interactors of lincRNAs in ES cells (66).

In flies, RNase A sensitive HP1 localization has been observed at transcriptionally induced heat shock protein loci (67). The same study also showed that the recruitment of HP1Y at these loci was dependent upon its interaction with nascent RNA. In S. 
pombe HP1 homologue, SWI6 interacts with RNA via its hinge region (68). Strikingly, its interaction with RNA leads to conformational change in the protein leading to the loss of its interaction with H3K9me3 (68). One RNA species known as 'Borderline' that is expressed from the boundary regions of pericentric heterochromatin and euchromatin interacts with SWI6. This leads to dissociation of SWI6 from chromatin, which prevents spreading of heterochromatin into neighboring euchromatin domains (60).

In summary, HP1 association to chromatin is determined by multiple factors such as CD, CSD, HP1 PTMs, RNA and so on. However, it is still not clear how H3K9me3 readout by HP1 proteins is regulated in vivo and is translated into heterochromatin organization and transcription regulation.

Although in yeast, several studies have been carried out in this direction (203), yeast SWI6 behaves differently compared to other HP1 proteins owing to the presence of long $\mathrm{N}$-terminal tail. In higher organisms such as flies and mammals, presence of multiple $\mathrm{HP} 1$ isoforms has hindered the understanding of HP1-H3K9me3 interaction in vivo. We therefore argued that $C$. elegans would provide a suitable model system for this study in which there exist two isoforms of HP1, namely HPL-1 and HPL-2, for both of which, null alleles are viable. 


\subsection{C. elegans as a model system}

C. elegans possesses several attractive features that make it a well-suited model organism for the study of genetics, development, neurobiology, cell biology and behavior $(70,71)$. It can be easily grown in the laboratory on an agar plate with a bacterial lawn or in liquid culture with concentrated bacteria added as its food (69). It's a free living, transparent nematode with small size (about $1.3 \mathrm{~mm}$ in length and $80 \mu \mathrm{m}$ in diameter). The invariant anatomy and invariant patterns of development among individuals has allowed the identification of exact cell lineages from a single-cell embryo to a fully-grown adult (959 cells in hermaphrodites and 1031 cells in male).

C. elegans has a generation time of 2-4 days, going from the single-cell embryo to an adult depending on the temperature conditions ( 2 days at $25^{\circ} \mathrm{C}$ and 4 days at $15^{\circ} \mathrm{C}$ ) (75). After hatching, the embryo goes through four larval stages before turning into a young adult. Each larval form appears similar to the adult, only smaller in size. C. elegans can acquire a more quiescent alternative life form, called a dauer larva, in response to overcrowding or the absence of adequate bacterial food. This dauer larva can remain viable for as long as three months, while it roams around in search of food. This allows maintenance of worms until required. Upon addition of food, dauer can resume its growth and within 10-12 hrs., it can reach L4 stage. The common form of adult is a hermaphrodite although males occur naturally at a frequency of $10^{-3}(70)$.

The genome of $C$. elegans is small compared to humans (about 30 times smaller), yet it encodes over 22,000 proteins, only slightly fewer than humans. About $35 \%$ of $C$. elegans genes are closely related to human genes (72-74). Several genetic screens in C. elegans have led to the identification of proteins that are required for various cellular processes. This provides a useful resource for molecular biological studies to understand molecular mechanism(s) of protein function.

\subsection{Chromatin regulation in C. elegans}

Histones are conserved from yeast to mammals. C. elegans histone $\mathrm{H} 3$ and $\mathrm{H} 4$ are $97 \%$ and $98 \%$ identical to their human counterparts respectively (76). Several of the histone modifications known in mammalian cells have also been experimentally detected in $C$. elegans. Their existence can also be predicted based on 1) sequence 
conservation and 2) presence of homologues of human chromatin modifying enzymes. Several of these enzymes have been characterized genetically and to some extent biochemically. These are involved in large number of cellular and developmental processes (76). Interestingly, C. elegans germline possesses a unique chromatin modification pattern that is crucial for global repression of the genome in the germline and serve as a guardian against mutations (77).

\subsubsection{Epigenetic features of $C$. elegans chromosomes}

Gene expression studies in C. elegans have revealed that chromosomal central regions have higher levels of gene expression compared to distal chromosomal arms (spans 4 $\mathrm{MB}$, on each side). Also, central regions consist of more essential genes whereas the chromosomal arms consist of more repetitive sequences. Arms are known to possess higher recombination frequency (78).

As far as epigenetic features are concerned, compared to central regions, arms are enriched for repressive histone marks such as H3K9me3/2/1 (figure 1.7) (79). Arms also show enrichment for LEM-2, a nuclear envelope protein suggesting the preferential association of arms to nuclear lamina. In contrast, central regions show higher enrichment for RNA pol II, H3K36me3 and H3K79me3 consistent with higher gene expression (figure 1.7) (79).

In spite of this demarcation, there exist actively expressed genes in chromosomal arms and possess gene profile plot that resemble to the highly expressed genes in central region (79). This observation has led to the speculation that these broad repressive chromosomal arms with islands of actively transcribed genes could operate via small chromatin loops that emerge from nuclear lamina (79).

This suggests that the arrangement of genes and repetitive sequences on chromosomes in $C$. elegans have evolved in order to accommodate the principles of epigenetic regulation and vice versa. 


\begin{tabular}{ccc}
$\begin{array}{c}\text { Distal chromosomal region } \begin{array}{c}\text { Or } \\
\text { Chromosomal arms }\end{array} \\
\text { Central region }\end{array}$ & $\begin{array}{c}\text { Distal chromosomal region } \\
\text { Or }\end{array}$ \\
\hline & & \\
\hline & & \\
\hline Chromosomal arms
\end{tabular}

Figure 1.7: Epigenetic features of $C$. elegans chromosomes

Various chromosomal regions, distal chromosomal arms (marked in red) and central region (marked in blue) are distinguished based on their distinct epigenetic features.

\subsection{Heterochromatin function in C. elegans}

In general, euchromatin and heterochromatin is spatially segregated into the nucleus and marked by boundary elements. Heterochromatin is highly enriched for H3K9me3 and HP1 proteins (203). In C. elegans, distal chromosomal arms associate with the nuclear lamina and form lamina associated domains (LADs). These are also rich in H3K9me3 and H3K27me3 (78). These regions harbor tissue-specific developmentally regulated genes/repetitive sequences that are transcriptionally silent regions of chromatin and occupy nuclear periphery (81). Repetitive transgene arrays are known to form heterochromatin, are rich in repressive histone marks and occupy nuclear periphery (81).

Interestingly, it has been shown that $\mathrm{H} 3 \mathrm{~K} 9 \mathrm{me} 3$ is dispensable for heterochromatin formation in C. elegans (44). Authors have shown that, worms can fully develop even in complete absence of the $\mathrm{H} 3 \mathrm{~K} 9 \mathrm{me} 3$ mark. This is in correlation to a recent study in mammals, stating that $\mathrm{H} 3 \mathrm{~K} 9 \mathrm{me} 1$ is required for heterochromatin formation while $\mathrm{H} 3 \mathrm{~K} 9 \mathrm{me} 3$ is dispensable for heterochromatin formation (45). Overall, these findings 
question the role of $\mathrm{H} 3 \mathrm{~K} 9 \mathrm{me} 3-\mathrm{HP} 1$ interaction in heterochromatin function.

\subsubsection{Role of HP1 proteins in heterochromatin function in C. elegans}

In C. elegans, there are two isoforms of HP1, namely HPL-1 (heterochromatin protein like-and HPL-2 (heterochromatin protein like-2). Of these two isoforms, HPL-2 has been shown to be required for vulva development and fertility, while HPL-1 functions redundantly to HPL-2 (104). Immunofluorescence analysis in early embryos suggests that HPL-2 and HPL-1 foci partially overlap with each other (103). Null mutant of hpl-2 shows increased transcription from heterochromatic regions while $h p /-1$ null mutant have no effect on transcription of heterochromatic regions (45). None of these mutants have any structural effect on heterochromatin organization (45). In agreement with this, another study has shown that in C. elegans, centromere formation occurs independent of HPL-2. Moreover, hpl-2 null mutant shows increased transcription of germline genes in somatic tissues (105). This could mean that HPL-2 is not a structural component of heterochromatin but is rather required only to repress aberrant transcription. However, the mechanisms of recruitment of HPL-2 to heterochromatic regions are unknown.

\subsection{Mechanism(s) of HPL-2 recruitment to chromatin}

\subsubsection{HP1-H3K9me3 interaction in C. elegans}

Since, heterochromatin formation occurs independent of H3K9me3 in C. elegans (45), recruitment of HPL-2 to heterochromatin may not be dependent upon H3K9me3. Genomes wide binding studies have shown that H3K9me3 poorly co-distributes with HPL-2 (83). Obviously, additional mechanisms are in place to recruit HPL-2 to heterochromatic regions.

\subsubsection{Role of CSD in recruitment of HPL-2 to chromatin}

Like other HP1 proteins, HPL-2 might also be recruited to chromatin via its CSD. CSD of HP1 proteins plays important role in protein-protein interactions. HPL-2 CSD is known to interact with a zinc-finger protein LIN-13 via PXVXL motif in vitro(117) 


\subsubsection{LIN-13, a zinc finger protein}

lin-13 is a member of the lin-35Rb class of genes involved in vulva development (96). $\mathrm{LIN}-13$ is a 2248-residue nuclear protein. It contains 24 zinc-fingers of the $\mathrm{C} 2 \mathrm{H} 2$ type and is a homologue of $\mathrm{Rb}$-like protein (96). Zinc fingers have been implicated in binding to DNA, RNA, proteins and even lipid substrates (143-146). Although no molecular function(s) have been described for LIN-13 as such, it is expressed in a variety of cell types from embryos, L3 stage hyp7 cells, and intestinal cells to adults (96).

Subcellular localization indicates that LIN-13 localizes to nucleus and form nuclear speckles consistent with its proposed role in transcriptional regulation. The speckles or discrete foci observed for LIN-13-GFP are similar to the ones observed in a study showing ELT-2-GFP binding to a transgenic array carrying ELT-2 binding sequence in living embryos (147). This raises an interesting possibility of LIN-13 binding to specific DNA sequences in the genome. LIN-13 also contains LXCXE motif that is found in many $\mathrm{Rb}$-interacting proteins and is essential for their interaction with $\mathrm{Rb}$ (149).

LIN-13 has been shown to be required for vulva development and fertility in C. elegans (96). It was identified originally in genetic screens for vulval mutants, and displays temperature sensitive multivulva (muv) phenotype. lin-13 null mutants at $25^{\circ} \mathrm{C}$ are muv and sterile whereas at $15^{\circ} \mathrm{C}$, they are sterile but not muv, making them non-viable (96). LIN-13 has been identified as an interaction partner of HPL-2 in yeast two-hybrid assay. Further, knock-down of lin-13 leads to delocalization of HPL-2-GFP foci in embryos suggesting that LIN-13 could recruit HPL-2 to chromatin (117). Another study has reported that LIN-13, HPL-2 and LIN-61 form a protein complex involved in the repression of germline RNAi in somatic tissues (105).

\subsubsection{LIN-61, an MBT repeat protein}

LIN-61 encodes for an MBT repeat containing protein, a homologue of the mammalian L3MBTL3 protein, involved in the readout of histone methylation. However, contrary to its mammalian homologue L3MBTL3, which recognize exclusively H4K20me1, LIN-61 preferentially binds to $\mathrm{H} 3 \mathrm{~K} 9 \mathrm{me} 3$ and $\mathrm{H} 3 \mathrm{~K} 9 \mathrm{me} 2$ (97). Furthermore, it has been shown that binding of LIN-61 to H3K9me3 in vivo is essential for vulva development (97). In contrast, another MBT repeat containing protein in C. elegans, MBTR-1 is not required for vulva development (97). 
Interestingly, null mutant of lin-61 shows increased transcription of heterochromatic regions although no structural defects are observed. Similar increase in transcription has been observed in hpl-2 null mutants (45). Moreover, double mutants of $h \mathrm{pl}-2$ and lin-61 show genetic interaction during vulva development. The double mutant also shows defects in heterochromatin organization, where heterochromatin positioning to nuclear periphery is lost $(45,97)$. Immunofluorescence analysis has revealed that LIN61 co-localizes with HPL-2 in embryos (105). The protein complex comprised of LIN-61, LIN-13 and HPL-2 has been reported (105). However, biochemical nature of this complex is unknown and it remains to be seen whether this protein complex has any role in the recruitment of HPL-2 to chromatin and thereby heterochromatinorganization.

\subsubsection{Role of RNA in recruitment of HPL-2 to chromatin}

Several HP1 proteins have been shown to interact with RNA molecules that modulate their association with chromatin (1.5.3). So far, there is no evidence for direct interaction of HPL-2 with RNA. However, HPL-2 has been identified as a regulator of RNAi pathways in C. elegans (215). It is therefore, possible that it associates with non-coding RNAs. It has been shown that HPL-2 is required for NRDE-3-mediated endogenous nuclear RNAi pathway that promotes chromatin silencing (84). Another study showed that HPL-2 is required for multigenerational silencing in the germline, mediated by piwiRNAs (122).

In summary, HPL-2 recruitment to chromatin could be mediated by any or combinations of the abovementioned possible mechanisms. We propose that these mechanisms ensure proper chromatin organization during vulva development and germline development in C. elegans, the physiological function attributed to $\mathrm{hpl}-2$.

\subsection{Vulva development and regulation in C. elegans}

In $C$. elegans, the vulva is derived from the descendants of three out of six equivalent vulval precursor cells (VPCs) named as P5.p, P6.p and P7.p. (figure 1.8). The remaining three VPCs (P3.p, P4.p and P8.p) acquire a cell fate that gives rise to nonvulval cells, which fuse to the hypodermal syncytium $(86,87)$. This vulval induction in $C$. elegans occurs at L3 stage, where a paracrine factor known as LIN-3, released by the 
anchor cell, binds to LET-2, a receptor tyrosine kinase present on the surface of VPCs (89-92). This leads to activation of RAS/MAPK signaling in VPCs giving rise to vulval fate.

For correct vulva development, it is crucial that the dose of LIN-3 that each VPC receives is fine-tuned. P6.p, which spatially lies just beneath the anchor cell, receives maximum signal giving rise to $1^{\circ}$ vulva fate, P5.p and P7.p receive intermediate levels of LIN-3 giving rise to $2^{\circ}$ vulva fate, whereas other three VPCs receive no LIN-3 signal, acquiring non-vulval fate $(88,89)$. lin-3 loss of function mutation leads to vulvaless phenotype whereas lin-3 gain of function or let-23 constitutively active dominant negative mutant gives rise to muv phenotype (90-93). Muv phenotype refers to one functional vulva apart from other ventral protrusions (pseudovulvae) due to acquisition of vulva fate by more than three VPCs.

Apart from mutations that directly influence VPC signaling, several other mutants give rise to muv phenotype. Most of these genes negatively regulate vulva development meaning that they normally antagonize vulval induction in VPCs. Such genes are known as SynMuv (synthetic multivulva) genes (94). Genetic studies have identified two functionally redundant sets of genes, as SynMuv A and SynMuv B. These refer to SynMuv A and SynMuv B pathway of vulva development, respectively (94). Worms having simultaneous mutations in both SynMuv $A$ and $B$ pathway develop multivulva phenotype (figure 1.8B). On the other hand, worms with one/more mutations in the same pathway develop WT vulva (figure 1.8A). The lin-15 locus is one of the earliest known regulators of vulva development (95). 


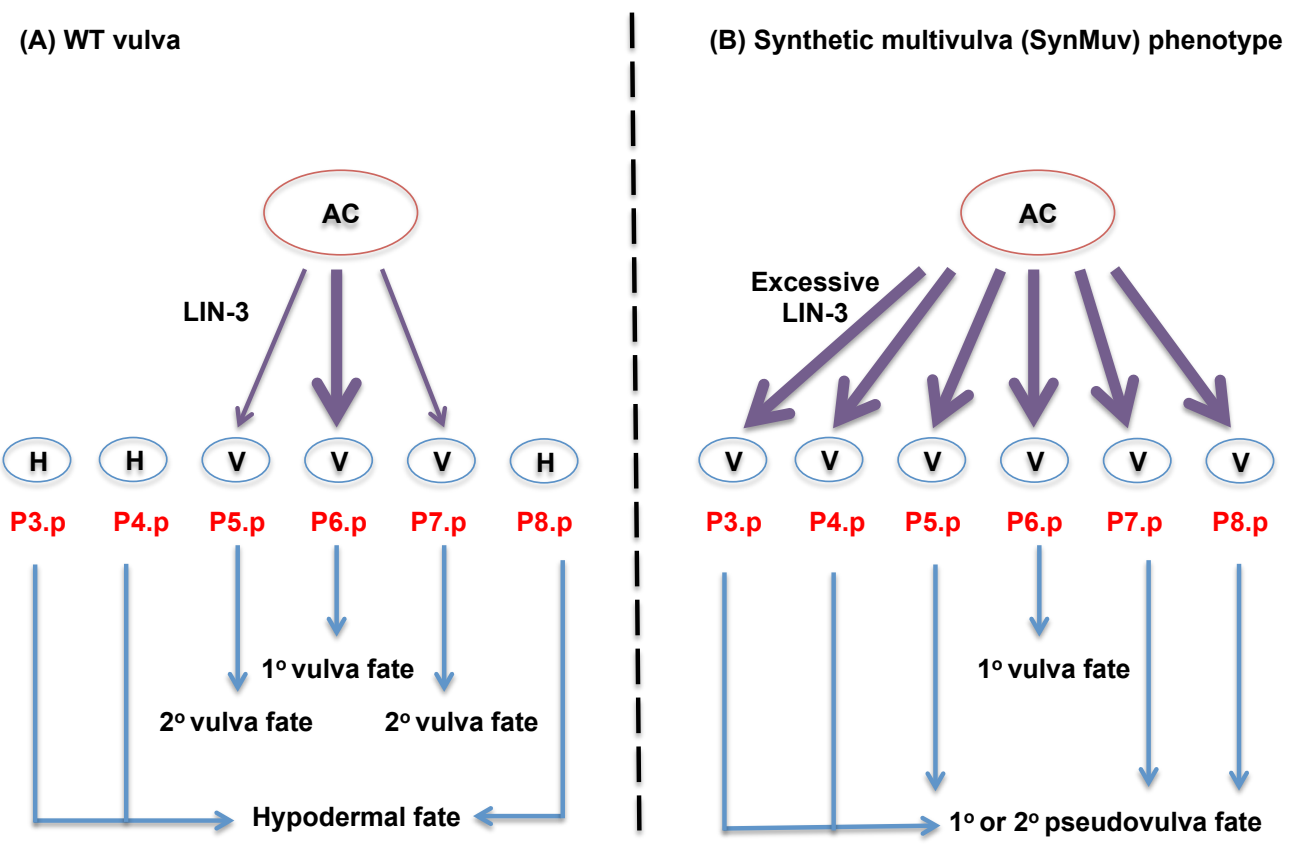

\section{Figure 1.8: Vulval induction signaling in C. elegans}

(A) Anchor cell (AC) which spatially lies just above P6.p VPC, releases paracrine factor, LIN-3 that leads to acquisition of $1^{\circ}$ vulva fate by P6.p and $2^{\circ}$ vulva fate by P5.p and P7.p in WT worms. (B) AC releases excessive LIN-3 that leads to acquisition of vulval fate by all six VPCs (from P3.p to P8.p) in SynMuv worms.

It encodes two genes, lin-15a and lin-15b that belong to SynMuv A and SynMuv B pathway respectively (95). Worms with simultaneous mutations in lin-15a and lin-15b develop muv phenotype whereas single mutants develop WT vulva. Since this finding, several SynMuv A genes such as lin-8, lin-56 and SynMuv B genes such as hpl-2, lin61, lin-9, lin-35, lin-37 have been identified (90).

Interestingly, there exist exceptions to this rule. lin-13, alone can cause highly penetrant muv phenotype (96), whereas several SynMuv B genes can cause highly penetrant muv phenotype in other SynMuv B mutant background (98). hpl-2;lin-61, hpl-2;met-2, met1;met-2 are some of the double mutants of SynMuv B class that cause severe muv phenotype (97). Based on these observations, it was proposed that SynMuv B genes act redundant to each other (97). 


\subsection{Objectives of the presented thesis}

Chromatin organization in eukaryotes is fundamentally linked to genome transcription. In order to initiate and maintain tissue-specific gene expression patterns, cellular mechanisms termed as 'epigenetic regulation' operate at the level of chromatin. These involve interplay among chromatin modifications, chromatin associated proteins, enzymes and non-coding RNAs. These ensure proper chromatin organization and function.

Heterochromatin formation and its maintenance is a major epigenetic regulatory mechanism and is crucial for tissue specific gene expression programs and cellular identity. Heterochromatin is a transcriptionally silent, highly condensed part of the genome. Our understanding of heterochromatin function is derived from studies in $S$. pombe and flies. Heterochromatin is rich in repetitive DNA elements, histone H3K9me3 modification and HP1 proteins. It has been proposed that HP1 proteins bind H3K9me3 deposited by SUV39H enzymes via their $\mathrm{N}$-terminal chromodomain and that the HP1$\mathrm{H} 3 \mathrm{~K} 9 \mathrm{me} 3$ interaction is required for heterochromatin organization and transcriptional repression.

However, recent studies have demonstrated active transcription in heterochromatic regions and recruitment of RNA pol II by heterochromatin factors (203). Also, H3K9me3 and HP1 proteins have been found on subsets of actively transcribing genes (203). This multifaceted nature of HP1 function and its role in opposing transcriptional activities has led us to pursue the complex nature of HP1-chromatin association.

All mammalian and Drosophila isoforms of HP1 bind H3K9me3 in vitro. However, their interaction with $\mathrm{H} 3 \mathrm{~K} 9 \mathrm{me} 3$ in vivo appears to be differentially regulated, as not all HP1 isoforms co-localize with $\mathrm{H} 3 \mathrm{~K} 9 \mathrm{me} 3$. Also, not all of these isoforms depend upon H3K9me3 for all of their functions. Although, S. pombe has provided substantial information about how HP1 operates to regulate heterochromatic transcription, it has substantial sequence divergence compared to mammals.

We proposed that in order to gain mechanistic insights into the role of HP1 in heterochromatin function and especially in transcription, it is essential to understand the mode of recruitment of HP1 proteins to chromatin. 
We reasoned that $C$. elegans provides a unique model system to study HP1-chromatin association due to the non-redundant nature of one of its homologues, HPL-2 and the easily identifiable phenotype that it is associated with. C. elegans also provides a suitable toolbox for combining genetic and biochemical approaches in order to delineate the exact mechanism(s) of HP1-chromatin association and their biological significance.

The objective of this thesis was to understand how various factors regulate HPL-2 association to chromatin and to uncover the biological significance of this regulation. We aimed to analyze the involvement and contribution of histone modification ( $\mathrm{H} 3 \mathrm{~K} 9 \mathrm{me} 3)$, protein interaction partners of HPL-2 (LIN-13 and LIN-61) in the regulation of HP1chromatin association.

We aimed to study-

1. Role of H3K9me3 in recruitment of HPL-2 to chromatin: In order to test the proposed function of $\mathrm{H} 3 \mathrm{~K} 9 \mathrm{me} 3$ in recruitment of HP1 to chromatin, we aimed to define the binding properties of HPL-2 to H3K9me3 in vitro and in vivo. Based on these, we aimed to understand whether $\mathrm{H} 3 \mathrm{~K} 9 \mathrm{me} 3$ plays an important role in HPL2-chromatin association and its function.

2. Role of LIN-61 and LIN-13 in HPL-2-H3K9me3 association: Since, it is proposed that HPL-2 is primarily associated with chromatin in H3K9me3 dependent manner, our goal was to test whether LIN-61 and LIN-13 influence its ability to interact with H3K9me3 and chromatin. We decided to undertake combination of genetic and biochemical approaches to test the effect of loss of LIN-61 and LIN-13 on H3K9me3 and chromatin association of HPL-2.

3. Define the chromatin association of HPL-2 with H3K9me3, LIN-61 and LIN-13 and its role in transcription regulation: We aimed to determine the co-occupancy of H3K9me3, LIN-61 and LIN-13 with HPL-2 on chromatin. Using genetic approaches, we aimed to understand whether loss of these factors has any effect on the transcription regulation of genes directly bound by these factors. In other words, our goal was to identify the direct transcriptional targets of HPL-2 and 
associated factors.

4. To understand the biological significance of HPL-2 association to chromatin: Based on the direct transcriptional targets, we aimed to identify the genes directly regulated by HPL-2 and its associated factors during vulva development and germline development in C. elegans. 


\section{Materials and methods}

\subsection{Materials}

\subsubsection{Laboratory equipment}

Generally used laboratory equipment is listed in table 2-1.

Table 2.1: Generally used laboratory equipment

\begin{tabular}{|c|c|}
\hline Equipment & Supplier \\
\hline Balances & Mettler-Toledo, Giessen, Germany \\
\hline Bioruptor $^{\mathrm{IM}}$ & Diagenode, Liege, Belgium \\
\hline Centrifuge sorwall evolution RC & $\begin{array}{l}\text { Thermo Fisher Scientific, Rockford, } \\
\text { USA }\end{array}$ \\
\hline Centrifuges 5415R / 5810R & Eppendorf, Hamburg, Germany \\
\hline EmulsiFlex-C5 high pressure homogenizer & Avestin, Ottawa, Canada \\
\hline $\begin{array}{l}\text { PCR machine eppendorf mastercycler } \\
\text { epgradient S }\end{array}$ & Eppendorf, Hamburg, Germany \\
\hline Freezer $-20^{\circ} \mathrm{C}$ & Liebherr, Bulle, Switzerland \\
\hline Freezer $-80^{\circ} \mathrm{C}$ & $\begin{array}{l}\text { Thermo Fisher Scientific, Rockford, } \\
\text { USA }\end{array}$ \\
\hline Gel documentation system & Bio-Rad, Munich, Germany \\
\hline Hera safe sterile hood & Heraeus, Hanau, Germany \\
\hline Hereaus kelviton incubator & $\begin{array}{l}\text { Thermo Fisher Scientific, } \\
\text { Rockford, USA }\end{array}$ \\
\hline Kodak X OMAT 2000 processor & Carestream Health, New York, USA \\
\hline Mini-PROTEAN electrophoresis system & Bio-Rad, Munich, Germany \\
\hline Mini-trans-blot system & Bio-Rad, Munich, Germany \\
\hline Multitron shaker & HT Infors, Braunschweig, Germany \\
\hline NanoDrop ${ }^{\circledR}$ ND-1000 & Peqlab, Erlangen, Germany \\
\hline
\end{tabular}




\begin{tabular}{|c|c|}
\hline Inverted research microscope, ECLIPSE Ti-E & Nikon, Kingston, UK \\
\hline Olympus SZX10 & Olympus, Hamburg, Germany \\
\hline $\mathrm{pH}$ meter & Mettler-Toledo, Giessen, Germany \\
\hline Power supply powerpack universal & Bio-Rad, Munich, Germany \\
\hline Scanner perfection V750 pro & Epson, Meerbusch, Germany \\
\hline $\begin{array}{l}\text { Sub-Cell-GT agarose gel electrophoresis } \\
\text { System }\end{array}$ & Bio-Rad, Munich, Germany \\
\hline Thermomixer comfort & Eppendorf, Hamburg, Germany \\
\hline UV transilluminator & Bio-Rad, Munich, Germany \\
\hline Vortex genie 2 & $\begin{array}{l}\text { Scientific Industries, Schwerte, } \\
\text { Germany }\end{array}$ \\
\hline Water bath TW12 & Julabo, Seelbach, Germany \\
\hline $\begin{array}{l}\text { Real-time PCR machine DNA engine } \\
\text { OPTICON }\end{array}$ & Bio-Rad, Munich, Germany \\
\hline Illumina HiSeq2000 TM & Illumina, Cambridge, UK \\
\hline
\end{tabular}




\subsubsection{Chemicals}

Chemicals used for buffers and media are listed in table 2-2.

Table 2.2: Generally used chemicals

\begin{tabular}{|l|l|}
\hline \multicolumn{1}{|c|}{ Chemical } & \multicolumn{1}{c|}{ Manufacturer } \\
\hline 100 mM dNTP set & Invitrogen, Karlsruhe, Germany \\
\hline 2-Mercaptoethanol & $\begin{array}{l}\text { Sigma-Aldrich, Steinheim, } \\
\text { Germany }\end{array}$ \\
\hline $\begin{array}{l}\text { 4-(2-Hydroxyethyl)-1-piperazineethanesulfonic } \\
\text { acid (HEPES) }\end{array}$ & VWR, Poole, UK \\
\hline Acetic acid & Merck, Mannheim, Germany \\
\hline Acrylamide:bisacrylamide (37.1:1) & Merck, Mannheim, Germany \\
\hline Agar & Roth, Karlsruhe, Germany \\
\hline Agarose & Serva, Heidelberg, Germany \\
\hline Ammonium chloride $\left(\mathrm{NH}_{4} \mathrm{Cl}\right)$ & Merck, Mannheim, Germany \\
\hline Ammonium persulfate $(\mathrm{APS})$ & $\begin{array}{l}\text { AppliChem GmbH, Darmstadt, } \\
\text { Germany }\end{array}$ \\
\hline Ampicillin & $\begin{array}{l}\text { AppliChem GmbH, Darmstadt, } \\
\text { Germany }\end{array}$ \\
\hline Benzamidine & $\begin{array}{l}\text { Sigma-Aldrich, Steinheim, } \\
\text { Germany }\end{array}$ \\
\hline Boric acid $\left(\mathrm{H}_{3} \mathrm{BO}_{3}\right)$ & $\begin{array}{l}\text { Merck, Mannheim, Germany } \\
\text { Albumin, from bovine serum }(\mathrm{BSA})\end{array}$ \\
\hline Bromophenol blue & $\begin{array}{l}\text { Sigma-Aldrich, Steinheim, } \\
\text { Germany }\end{array}$ \\
\hline Calcium chloride $\left(\mathrm{CaCl}_{2}\right)$ & Serva, Heidelberg, Germany \\
\hline Chloroform & Roth, Karlsruhe, Germany \\
\hline Cholesterol & Merck, Mannheim, Germany \\
\hline
\end{tabular}




\begin{tabular}{|l|l|}
\hline Citric acid monohydrate & Alfar Aesar, Karlsruhe, Germany \\
\hline Cobalt chloride $\left(\mathrm{CoCl}_{2}\right)$ & $\begin{array}{l}\text { Riedel-de Haen, Seelze, } \\
\text { Germany }\end{array}$ \\
\hline Coomassie brilliant blue & Bio-Rad, Munich, Germany \\
\hline Copper chloride $\left(\mathrm{CuCl}_{2}\right)$ & Merck, Mannheim, Germany \\
\hline Copper sulfate $\left(\mathrm{CuSO}_{4}\right)$ & Merck, Mannheim, Germany \\
\hline Dimethyl pimelimidate (DMP) & Pierce, Rockford, USA \\
\hline Dimethylsulfoxide (DMSO) & $\begin{array}{l}\text { Sigma-Aldrich, Steinheim, } \\
\text { Germany }\end{array}$ \\
\hline Dipotassium hydrogen phosphate (K $\left.{ }_{2} \mathrm{HPO} 4\right)$ & Roth, Karlsruhe, Germany \\
\hline Dithiothreitol (DTT) & $\begin{array}{l}\text { Alexis Biochemicals, Loerrach, } \\
\text { Germany }\end{array}$ \\
\hline Ethanol & Merck, Mannheim, Germany \\
\hline Ethanolamine & $\begin{array}{l}\text { Sigma-Aldrich, Steinheim, } \\
\text { Germany }\end{array}$ \\
\hline Ethidium bromide & Roth, Karlsruhe, Germany \\
\hline Ethylenediaminetetraacetate (EDTA) & Roth, Karlsruhe, Germany \\
\hline Gelatin from bovine & $\begin{array}{l}\text { Sigma-Aldrich, Steinheim, } \\
\text { Germany }\end{array}$ \\
\hline Glucose & Merck, Mannheim, Germany \\
\hline Glutathione reduced & $\begin{array}{l}\text { Sigma-Aldrich, Steinheim, } \\
\text { Germany }\end{array}$ \\
\hline Glycerol & Merck, Mannheim, Germany \\
\hline Glycine & Merck, Mannheim, Germany \\
\hline Hydrochloric acid 37\% (HCl) & Merck, Mannheim, Germany \\
\hline Imidazole & Roth, Karlsruhe, Germany \\
\hline Iron chloride (FeCl $\left.{ }_{3}\right)$ & Roth, Karlsruhe, Germany \\
\hline Isoamyl alcohol & $\begin{array}{l}\text { Sigma-Aldrich, Steinheim, } \\
\text { Germany }\end{array}$ \\
\hline Isopropyl beta-D-thiogalactopyranoside (IPTG) & AppliChem GmbH, Darmstadt, \\
\hline
\end{tabular}




\begin{tabular}{|c|c|}
\hline & Germany \\
\hline Kanamycin & $\begin{array}{l}\text { Sigma-Aldrich, Steinheim, } \\
\text { Germany }\end{array}$ \\
\hline Low fat dry milk & $\begin{array}{l}\text { Regilait, Saint Martin Belle- } \\
\text { Roche, France }\end{array}$ \\
\hline Magnesium chloride $\left(\mathrm{MgCl}_{2}\right)$ & Merck, Mannheim, Germany \\
\hline Magnesium sulfate $\left(\mathrm{MgSO}_{4}\right)$ & Roth, Karlsruhe, Germany \\
\hline D-(+)-Maltose monohydrate & $\begin{array}{l}\text { Sigma-Aldrich, Steinheim, } \\
\text { Germany }\end{array}$ \\
\hline Manganese chloride $\left(\mathrm{MnCl}_{2}\right)$ & Roth, Karlsruhe, Germany \\
\hline Methanol & $\begin{array}{l}\text { Sigma-Aldrich, Steinheim, } \\
\text { Germany }\end{array}$ \\
\hline Nickel chloride $\left(\mathrm{NiCl}_{2}\right)$ & $\begin{array}{l}\text { Riedel-de Haen, Seelze, } \\
\text { Germany }\end{array}$ \\
\hline Nonidet P-40 (NP-40) & Roche, Karlsruhe, Germany \\
\hline Nystatin & $\begin{array}{l}\text { AppliChem GmbH, Darmstadt, } \\
\text { Germany }\end{array}$ \\
\hline NZ-amine & Roth, Karlsruhe, Germany \\
\hline Peptone & Roth, Karlsruhe, Germany \\
\hline Phenol:Chlorofom:Isoamyl alcohol (25:24:1) & $\begin{array}{l}\text { Sigma-Aldrich, Steinheim, } \\
\text { Germany }\end{array}$ \\
\hline Phenylmethylsulfonyl fluoride (PMSF) & Serva, Heidelberg, Germany \\
\hline Potassium chloride $(\mathrm{KCl})$ & Roth, Karlsruhe, Germany \\
\hline Potassium dihydrogen phosphate $\left(\mathrm{KH}_{2} \mathrm{PO}_{4}\right)$ & Roth, Karlsruhe, Germany \\
\hline Sodium acetate & Roth, Karlsruhe, Germany \\
\hline Sodium azide $\left(\mathrm{NaN}_{3}\right)$ & Alfar Aesar, Karlsruhe, Germany \\
\hline Sodium dihydrogen phophate $\left(\mathrm{NaH}_{2} \mathrm{PO}_{4}\right)$ & Merck, Mannheim, Germany \\
\hline Sodium dodecyl sulfate (SDS) & VWR, Poole, UK \\
\hline Sodium hydroxide $(\mathrm{NaOH})$ & Merck, Mannheim, Germany \\
\hline Sodium hypochlorite $(\mathrm{NaClO})$ & Roth, Karlsruhe, Germany \\
\hline
\end{tabular}




\begin{tabular}{|c|c|}
\hline Sodium molybdate $\left(\mathrm{Na}_{2} \mathrm{MoO}_{4}\right)$ & Merck, Mannheim, Germany \\
\hline Sodium monohydrogen phosphate $\left(\mathrm{Na}_{2} \mathrm{HPO}_{4}\right)$ & Merck, Mannheim, Germany \\
\hline Sodium selenite $\left(\mathrm{Na}_{2} \mathrm{SeO}_{3}\right)$ & Alfar Aesar, Karlsruhe \\
\hline Sodium sulfate $\left(\mathrm{Na}_{2} \mathrm{SO}_{4}\right)$ & Merck, Mannheim, Germany \\
\hline Sodium chloride $(\mathrm{NaCl})$ & Merck, Mannheim, Germany \\
\hline Tetramethylethylenediamine (TEMED) & $\begin{array}{l}\text { Sigma-Aldrich, Steinheim, } \\
\text { Germany }\end{array}$ \\
\hline Trichloroacetic acid (TCA) & Merck, Mannheim, Germany \\
\hline Tris (hydroxymethyl) aminoethane (Tris) & Roth, Karlsruhe, Germany \\
\hline Triton $^{\circledR} \mathrm{X}-100$ & Merck, Mannheim, Germany \\
\hline TRIzol $^{(\otimes}$ Reagent & Invitrogen, Karlsruhe, Germany \\
\hline Tryptone & Roth, Karlsruhe, Germany \\
\hline Tween $^{\circledR}-20$ & $\begin{array}{l}\text { Sigma-Aldrich, Steinheim, } \\
\text { Germany }\end{array}$ \\
\hline Yeast extract & MOBIO, Hamburg, Germany \\
\hline Zink sulfate (ZnSO4) & $\begin{array}{l}\text { AppliChem GmbH, Darmstadt, } \\
\text { Germany }\end{array}$ \\
\hline$\alpha$-Lactose & Roth, Karlsruhe, Germany \\
\hline
\end{tabular}




\subsubsection{Consumables}

Generally used consumables and other reagents are listed in table 2-3.

Table 2.3: Generally used consumables and other reagents

\begin{tabular}{|l|l|}
\hline \multicolumn{1}{|c|}{ Consumables / Others } & \multicolumn{1}{c|}{ Supplier } \\
\hline $9 \mathrm{~cm}, 6 \mathrm{~cm}, 3.5 \mathrm{~cm}$ Petri dish & Greiner, Solingen, Germany \\
\hline Amersham hyperfilm ECL $(18 \times 24 \mathrm{~cm})$ & GE Healthcare, Buckinhamshire, UK \\
\hline Amicon ultra-15 centrifugal filter units & Millipore, Billerica, USA \\
\hline Cryotubes & Greiner, Solingen, Germany \\
\hline Dynabeads ${ }^{\circledR}$ M280 sheep anti rabbit IgG & Invitrogen, Karlsruhe, Germany \\
\hline GeneRuler ${ }^{\text {TM }} 1$ kb plus DNA ladder & Fermentas, St. Leon-Rot, Germany \\
\hline Glutathione sepharose 4 fast flow & GE Healthcare, Buckinhamshire, UK \\
\hline Ni-NTA resin & Qiagen, Hilden, Germany \\
\hline Nitrocellulose membrane & GE Healthcare, Buckinhamshire, UK \\
\hline Phase lock Gel ${ }^{\text {TM }}$ (2 ml, heavy) & Eppendorf, Hamburg, Germany \\
\hline Pierce ${ }^{\circledR}$ avidin agarose resin & $\begin{array}{l}\text { Thermo Fisher Scientific, Rockford, } \\
\text { USA }\end{array}$ \\
\hline EDTA-free complete protease inhibitor & Roche, Penzberg, Germany \\
\hline cocktail $^{\text {SeeBlue }}{ }^{\circledR}$ plus2 pre-stained protein standard & Invitrogen, Karlsruhe, Germany \\
\hline
\end{tabular}




\subsubsection{Kits}

Generally used kits are listed in table 2-4.

Table 2.4: Generally used kits

\begin{tabular}{|l|l|}
\hline \multicolumn{1}{|c|}{ Kits } & \multicolumn{1}{c|}{ Supplier } \\
\hline 2x iQ SYBR ${ }^{\circledR}$ green supermix & Bio-Rad, Munich, Germany \\
\hline Coomassie plus (Bradford) protein Assay & $\begin{array}{l}\text { Thermo Fisher Scientific, Rockford, } \\
\text { USA }\end{array}$ \\
\hline ECL plus western blotting detection reagents & GE Healthcare, Buckinhamshire, UK \\
\hline Micro BSA protein assay kit & $\begin{array}{l}\text { Thermo Fisher Scientific, Rockford, } \\
\text { USA }\end{array}$ \\
\hline NucleoBond ${ }^{\circledR}$ Xtra midi & $\begin{array}{l}\text { Macherey \& Nagel, Dueren, } \\
\text { Germany }\end{array}$ \\
\hline NucleoBond ${ }^{\circledR}$ Gel extraction kit & $\begin{array}{l}\text { Macherey \& Nagel, Dueren, } \\
\text { Germany }\end{array}$ \\
\hline QIAprep ${ }^{\circledR}$ spin miniprep & Qiagen, Hilden, Germany \\
\hline $\begin{array}{l}\text { SuperScript III first-strand synthesis system for } \\
\text { RT-PCR }\end{array}$ & Invitrogen, Karlsruhe, Germany \\
\hline Maxima H minus cDNA synthesis kit & $\begin{array}{l}\text { Thermo Fisher Scientific, Rockford, } \\
\text { USA }\end{array}$ \\
\hline $\begin{array}{l}\text { TN } T^{\circledR} T^{17} \text { quick coupled transcription/ translation } \\
\text { System }\end{array}$ & Promega, Madison, USA \\
\hline Low input quick amp labeling kit, & Agilent, Waldbronn, Germany \\
\hline Agilent Gene Expression hybridization kit & Agilent, Waldbronn, Germany \\
\hline TruSeq RNA Library preparation kit & Illumina, Cambridge, UK \\
\hline & \\
\hline
\end{tabular}




\subsubsection{Enzymes}

Generally used enzymes are listed in table 2-5.

Table 2.5: Generally used enzymes

\begin{tabular}{|l|l|}
\hline \multicolumn{1}{|c|}{ Enzyme } & \multicolumn{1}{c|}{ Supplier } \\
\hline Antarctic phosphatase & New England Biolabs (NEB), Ibswich, USA \\
\hline BamHI, Ncol, Ndel, Xhol, EcoRI & New England Biolabs (NEB), Ibswich, USA \\
\hline DNasel & New England Biolabs (NEB), Ibswich, USA \\
\hline Dpnl & New England Biolabs (NEB), Ibswich, USA \\
\hline Pfu ultraTM II fusion HS DNA polymerase & Stratagene, La Jolla, USA \\
\hline Proteinase K & Invitrogen, Karlsruhe, Germany \\
\hline T4 DNA ligase & New England Biolabs (NEB), Ibswich, USA \\
\hline Taq Pol mix & Thermo Scientific, Rockford, USA \\
\hline RNase A & Qiagen, Hilden, Germany \\
\hline
\end{tabular}

\subsubsection{Histone peptides}

Histone peptides (biotinylated at $C$ terminus) were synthesized at the NMR based structural laboratory at the Max Planck Institute of Biophysical Chemistry in Göttingen, Germany (MPI).

Table 2.6: Generally used biotinylated peptides

\begin{tabular}{|c|l|l|l|l|}
\hline Name & \multicolumn{1}{|c|}{ Histone (aa) } & \multicolumn{1}{|c|}{ Peptide sequence } & Modification & Supplier \\
\hline H3K9me0 & $\mathrm{H} 3(1-20)$ & $\begin{array}{l}\mathrm{NH}_{2-} \\
\text { ARTK4QTARKSTGGKAPRKQ } \\
-\mathrm{COOH}\end{array}$ & Unmodified & MPI \\
\hline
\end{tabular}




\begin{tabular}{|l|l|l|l|l|}
\hline H3K9me1 & $\mathrm{H} 3(1-20)$ & $\begin{array}{l}\mathrm{NH}_{2-} \\
\text { ARTKQTARK(me1)STGGKAP } \\
\text { RKQ-COOH }\end{array}$ & K9me1 & MPI \\
\hline H3K9me2 & H3 (1-20) & $\begin{array}{l}\mathrm{NH}_{2-} \\
\text { ARTKQTARK(me2)STGGKAP } \\
\text { RKQ-COOH }\end{array}$ & K9me2 & MPI \\
\hline H3K9me3 & H3 (1-20) & $\begin{array}{l}\mathrm{NH}_{2-} \\
\text { ARTKQTARK(me3)STGGKAP } \\
\text { RKQ-COOH }\end{array}$ & K9me3 & MPI \\
\hline
\end{tabular}

\subsubsection{Antibodies obtained from different sources}

Generally used antibodies are listed in table 2-7.

Table 2.7: Generally used antibodies

\begin{tabular}{|c|c|c|c|}
\hline Name & $\begin{array}{c}\text { Supplier; order } \\
\text { number }\end{array}$ & Source & $\begin{array}{c}\text { Dilution (for } \\
\text { Western blotting) }\end{array}$ \\
\hline \multicolumn{4}{|c|}{ Primary antibodies } \\
\hline$\alpha G F P$ & Roche, 11814460001 & $\begin{array}{l}\text { Mouse, } \\
\text { monoclonal }\end{array}$ & $1: 1000$ \\
\hline aGST tag & Santa cruz, Sc-138 & $\begin{array}{l}\text { Mouse, } \\
\text { monoclonal }\end{array}$ & $1: 1000$ \\
\hline$\alpha \mathrm{H} 3$ & Abcam, ab1791 & $\begin{array}{l}\text { Rabbit, } \\
\text { polyclonal }\end{array}$ & $1: 10000$ \\
\hline$\alpha \mathrm{HPL}-2$ & $\begin{array}{l}\text { Novus Biologicals, } \\
38630002\end{array}$ & $\begin{array}{l}\text { Rabbit, } \\
\text { polyclonal }\end{array}$ & $1: 2500$ \\
\hline aLIN-13 & $\begin{array}{l}\text { Novus Biologicals, } \\
29570002\end{array}$ & $\begin{array}{l}\text { Rabbit, } \\
\text { polyclonal }\end{array}$ & $1: 2000$ \\
\hline $\begin{array}{l}\text { aLIN-61 (guinea } \\
\text { pig) }\end{array}$ & (75) & $\begin{array}{l}\text { Guinea pig, } \\
\text { polyclonal }\end{array}$ & $1: 1000$ \\
\hline
\end{tabular}




\begin{tabular}{|l|l|l|l|}
\hline aLIN-61 & $(75)$ & $\begin{array}{l}\text { Rabbit, } \\
\text { polyclonal }\end{array}$ & $1: 1000$ \\
\hline aMyc tag & Millipore; 05-724 & $\begin{array}{l}\text { Mouse, } \\
\text { monoclonal }\end{array}$ & $1: 1000$ \\
\hline \multicolumn{3}{|c|}{ Secondary antibodies } \\
\hline anti-guinea pig-HRP & $\begin{array}{l}\text { DakoCytomation; } \\
\text { P0141 }\end{array}$ & $\begin{array}{l}\text { Rabbit, } \\
\text { polyclonal }\end{array}$ & $1: 5000$ \\
\hline anti-mouse-HRP & $\begin{array}{l}\text { DakoCytomation; } \\
\text { P0447 }\end{array}$ & Goat, polyclonal & $1: 5000$ \\
\hline anti-rabbit-HRP & $\begin{array}{l}\text { DakoCytomation; } \\
\text { P0399 }\end{array}$ & $\begin{array}{l}\text { Swine, } \\
\text { polyclonal }\end{array}$ & $1: 5000$ \\
\hline $\begin{array}{l}\text { Clean blot IP } \\
\text { detection reagent }\end{array}$ & Pierce; 21230 & - & $1: 1000$ \\
\hline
\end{tabular}

\subsubsection{Bacterial strains}

Bacterial strains used for cloning, expression of recombinant proteins or as food source for C. elegans are listed in table 2-8.

Table 2.8: Bacterial strains

\begin{tabular}{|l|l|l|l|}
\hline \multicolumn{1}{|c|}{ Strain } & \multicolumn{1}{|c|}{ Genotye/Description } & Application & \multicolumn{1}{c|}{ Source } \\
\hline DH5a & $\begin{array}{l}\text { E. coli } \\
\text { F- f80lacZDM15 D(lacZYA-argF) U169; deoR } \\
\text { recA1 endA1 hsdR17(rk-, mk+) phoAsupE44 } \\
\text { Ithi-1 gyrA96 relA1 }\end{array}$ & $\begin{array}{l}\text { Plasmid } \\
\text { amplification; } \\
\text { cloning }\end{array}$ & $\begin{array}{l}\text { Invitrogen, } \\
\text { Karlsruhe }\end{array}$ \\
\hline $\begin{array}{l}\text { BL21-Codon } \\
\text { Plus (DE3)- } \\
\text { RIL }\end{array}$ & $\begin{array}{l}\text { E. coli } \\
\text { B F- ompT hsdS (rB- mB-) dcm+ Tetr gal I } \\
\text { (DE3) endA Hte [argU ileY leuW Camr] }\end{array}$ & $\begin{array}{l}\text { Protein } \\
\text { expression }\end{array}$ & $\begin{array}{l}\text { Stratagene, } \\
\text { La Jolla } \\
\text { (USA) }\end{array}$ \\
\hline OP50-1 & $\begin{array}{l}\text { Uracil auxotroph. E. coli streptomycin } \\
\text { resistant, useful for growing C. elegans in bulk }\end{array}$ & $\begin{array}{l}\text { C. elegans } \\
\text { Food }\end{array}$ & \begin{tabular}{l} 
CGC, USA \\
\hline
\end{tabular}
\end{tabular}




\begin{tabular}{|l|l|l|l|}
\hline HB101 & $\begin{array}{l}\text { E. coli } \\
\text { This strain of E. coli is easier for worms to eat } \\
\text { than other E. coli strains. [supE44 hsdS20(rB- } \\
\text { mB-) recA13 ara-14 proA2 lacY1 galK2 rpsL20 } \\
\text { xyl-5 mtl-1]. Contains a mutation (rpsL20) in a } \\
\text { ribosomal subunit gene that confers } \\
\text { streptomycin resistance }\end{array}$ & $\begin{array}{l}\text { C. elegans } \\
\text { Food }\end{array}$ & CGC, USA \\
\hline HT115(DE3) & $\begin{array}{l}\text { E. coli } \\
\text { F-, mcrA, mcrB, IN(rrnD-rrnE)1, } \\
\text { rnc14::Tn10(DE3 lysogen: lavUV5 promoter - } \\
\text { T7 polymerase) (IPTG-inducible T7 } \\
\text { polymerase) (RNAse III minus), tetracycline } \\
\text { resistant }\end{array}$ & RNAi feeding & CGC, USA \\
\hline
\end{tabular}

(CGC stands for Caenorhabditis Genetics Center (CGC), which is funded by the NIH National Center for Research Resources (NCRR))

\subsubsection{Plasmids used in this study}

Plasmids used in this study are listed in table 2-9.

Table 2.9: Plasmids used in this study

\begin{tabular}{|l|l|l|l|l|l|}
\hline \multicolumn{1}{|c|}{ Name } & \multicolumn{1}{|c|}{ Type } & Promoter & Selection & Tag & \multicolumn{1}{c|}{ Source } \\
\hline pET16b & Bacterial & T7 & Ampicillin & His & $\begin{array}{l}\text { Merck, Mannheim, } \\
\text { Germany }\end{array}$ \\
\hline pGEX-4T3 & Bacterial & Tac & Ampicillin & GST & $\begin{array}{l}\text { GE healthcare, } \\
\text { Buckinhamshire, UK }\end{array}$ \\
\hline pCDNA3.1(+) & Mammalian & T7/CMV & Ampicillin & - & $\begin{array}{l}\text { Invitrogen, Karlsruhe, } \\
\text { Germany }\end{array}$ \\
\hline pL4440 & Worm & T7 & Ampicillin & - & Prof. Andrew Fire \\
\hline
\end{tabular}


2.1.10 C. elegans strains used in this study

C. elegans strains used in this study are listed in table 2-10.

Table 2.10: C. elegans strains used in this study

\begin{tabular}{|c|c|c|c|}
\hline Strain & Genotype & Source & Reference \\
\hline N2 & wild-type & CGC & (70) \\
\hline PFR40 & hpl-2(tm1489)III & CGC & (103) \\
\hline lin-61 (tm2649) & $\operatorname{lin}-61$ (tm2649)I & $\begin{array}{l}\text { National bioresource project } \\
\text { (NBRP) }\end{array}$ & (97) \\
\hline MT15643 & mbtr-1(n4775)I & Prof. Horvitz & (99) \\
\hline MT13293 & met-2(n4256)III & Prof. Horvitz & (103) \\
\hline MT1806 & $\operatorname{lin}-15 A(n 767) X$ & Prof. Horvitz & (90) \\
\hline PFR60 & hpl-1(tm1624)X & CGC & (103) \\
\hline $\begin{array}{l}\text { arls48 (gfp:::lin- } \\
\text { 13) }\end{array}$ & $\begin{array}{l}\text { arls48[GFP:::lin- } \\
13]\end{array}$ & Prof. Greenwald & (96) \\
\hline lin-61(n3809) & lin-61(n3809)l & Prof. Horvitz & (99) \\
\hline $\operatorname{lin}-61(n 3447) !$ & $\operatorname{lin}-61(n 3447) !$ & Prof. Horvitz & (99) \\
\hline$h p l-2:: g f p$ & $h p /-2:: g f p$ & Prof. Palladino & (104) \\
\hline hpl-1::gfp & hpl-1::gfp & Prof. Palladino & (104) \\
\hline lin-61::gfp & lin-61::gfp & Prof. Ruvkun & (105) \\
\hline lin-61::3Xflag & lin-61::3Xflag & Prof. Ruvkun & (105) \\
\hline lin-56 (n2728) & lin-56 (n2728) & CGC & $(218)$ \\
\hline $\operatorname{lin}-8$ (n2731) & lin-8 (n2731) & Prof. Horvitz & (99) \\
\hline
\end{tabular}


2.1.11 C. elegans strains generated in this study

C. elegans strains generated in this study are listed in table 2-11.

Table 2.11: C. elegans strains generated in this study

\begin{tabular}{|l|l|c|}
\hline \multicolumn{1}{|c|}{ Strain name } & Genotype & Generated by \\
\hline lin61::gfp; $h p l-2$ (tm1489) & lin61::gfp; $h p l-2$ (tm1489) & Crossing \\
\hline lin61::gfp; lin-61 (tm2649) & lin61::gfp; lin-61 (tm2649) & Crossing \\
\hline lin61::flag; $h p l-2 ~(t m 1489)$ & lin61::flag; $h p l-2$ (tm1489) & Crossing \\
\hline hpl-2::gfp; lin-61 (tm2649) & hpl-2:::gfp; lin-61 (tm2649) & Crossing \\
\hline hpl-2::gfp; $h p l-2 ~(t m 1489)$ & hpl-2:::gfp; $h p l-2$ (tm1489) & Crossing \\
\hline lin13::gfp; $h p l-2$ (tm1489) & lin13:::gfp; $h p l-2$ (tm1489) & Crossing \\
\hline lin13::gfp; lin-61 (tm2649) & lin13:::gfp; lin-61 (tm2649) & Crossing \\
\hline hpl-1::gfp; $h p l-2 ~(t m 1489)$ & $h p l-1:: g f p ; ~ h p l-2 ~(t m 1489)$ & Crossing \\
\hline
\end{tabular}

\subsubsection{Primers used in this study}

Primers used in this study are listed in table 2-12.

Table 2.12: Primers used in this study

\begin{tabular}{ll}
\hline Primer & \multicolumn{1}{c}{ Sequence } \\
\hline Primers used in cloning \\
\\
hpl-2del1FP: & 5'-CCGAATTCCATGTCGAGCAAATCAA-3' \\
hpl-2del1RP: & 5'-CCGCTCGAGTTATTCCCTCTCAAACTCGTCCAA-3' \\
hpl-2del2FP: & 5'-CCGAATTCCATGTCGAGCAAATCAA-3' \\
hpl-2del2RP: & 5'-CCGCTCGAGTTACTTTTCATCTTTATCCTCTTC-3' \\
hpl-2del3FP: & 5'-CCGAATTCCTTTTCAAAGAGAGAGAAACCA-3' \\
hpl-2del3RP: & 5'-CCGCTCGAGTTACTTTTCATCTTTATCCTCTTC-3' \\
hpl-2del4FP: & 5'-CCGAATTCCAAGGAAACGAATCAAAATGAC-3' \\
hpl-2del4RP: & 5'-CCGCTCGAGTTACTTTTCATCTTTATCCTCTTC-3'
\end{tabular}


Primers used in site-directed mutagenesis

hPl-2T29EFP:

5'-GGAAAAAGTGCTGGACAAGCGAGAGGGAAAAGCCGGCAGAGA-3'

hPl-2T29ERP:

5'-TCTCTGCCGGCTTTTCCCTCTCGCTTGTCCAGCACTTTTTC-3'

hPl-2S82EFP:

5'-CCAATTCGCAAACGACACGAGCAGAAGCCCGAACCTTCC-3'

hPl-2S82ERP:

5'-GGAAGGTTCGGGCTTCTGCTCGTGTCGTTTGCGAATTGG-3'

hpl-2T104EFP:

5'-GATAAAGATGAAAACAACCAAGAGAATCAAAATGACAAATTCTCACT-3'

hPl-2T104ERP:

5'-CCAGTGAGAATTTGTCATTTTGATTCTCTTGGTTGTTTTCATCTTTATC-3'

hPl-2S155EFP:

5'-TTCGTAGTATCTGATAACTTGCTCCGgATAGCGACTGTTCACCTC-3'

hpl-2S155ERP:

5'- GAGGTGAACAGTCGCTATCCGgAGCAAGTTATCAGATACTACGAA-3'

hpl-2F19AFP:

5'-GCTAGAGTCAGACTCGgGGGCTCCTTGCGCCTGTATGAGAAATTCGT-3'

hPl-2-F19ARP:

5'-CAGCACTTTTTCCACCATGGCCACGTTGTCCTTCGGATCT-3'

hpl-2W41AF44AFP: 5'-AGACGAATTTCTCATACAGGCGCAAGGAGCCCCCGAGTCTGACTCTA-3'

hpl-2W41AF44ARP: 5'-AGATCCGAAGGACAACGTGGCCATGGTGGAAAAAGTGCTG-3'

Primers used in single worm PCR

The following primers were used to analyze hermaphrodites for the lin-61 allele, lin$61(\operatorname{tm} 2649)$ :

lin sw for2id gr: 5'-GACAACGTCTTGAACTATTAAATTATTCC-3'

lin sw R1: 5'-GTGTGCTCAATATATTCCTTTTTCG-3'

lin-delmut-for: 5'-CGGACAGAAAACTCGATAAGACCTAC-3'

The following primers were used to analyze hermaphrodites for the hpl-2 allele, $h p l-2$ (tm1489):

sw-hpl2 for1: $\quad$ 5'-CTTCTGGCTGTGTCGTTTGCG-3'

sw-hpl2rev: 5'-CTGTACGGTGATTGTCTGTGAG-3'

sw-hpl2for2indel: $\quad$ 5'-GCTCGCTTTGTTGATTTGCTCG-3'

The following primers were used to analyze hermaphrodites for the hpl-2::gfp:

hpl2gfp fp: 5'-CGAGATGTTGGACGAGTTTGAGA-3'

hpl2gfp rp: 5'-CTGCTTGCCTTCCAGTGAGAATTTG-3'

gfp rp: 5'-CAAGAATTGgGACAACTCCAGTG-3' 
The following primers were used to analyze hermaphrodites for the lin-61::gfp:

swLG/lin61: 5'-GTTGGATTTGCAGCAGTCAATGG-3'

swLG/lin-61: 5'-GTTGAAGATTCATGTTCGATAGGTGC-3'

gfp rp: 5'-CAAGAATTGGGACAACTCCAGTG-3'

Primers used in real time RT-PCR

$\begin{array}{ll}\text { rpl-26qfwd: } & \text { 5'-AAAGAGCCGCAAGGCCCACTTC-3' } \\ \text { rpl-26qrev: } & \text { 5'-TGTTTCCCTTATGGCGTCCGCG-3' } \\ \text { cdc-42qfwd: } & \text { 5'-CCAGGAATGCTCGAGAAACTGGC-3' } \\ \text { cdc-42qrev: } & \text { 5'-TCCCTTCTGCGTCAACGCTGA-3' } \\ \text { y45f10d4qfwd: } & \text { 5'-TGCCTCATCTTCCCTGGCAACC-3' } \\ \text { y45f10d4qrev: } & \text { 5'-TGGCGTCTTGGGCGAGCATT-3' } \\ \text { lin-13qfwd: } & \text { 5'-TGCGTCGAAGGCGTTGTGTG-3' } \\ \text { lin-13qrev: } & \text { 5'-TCGAGCCCGTGGCGTATGAT-3' } \\ \text { lin-15bqfwd: } & \text { 5'-CGGCGAAGATTGCGAATACGT-3' } \\ \text { lin-15bqrev: } & \text { 5'-ATCTTTGCGCGGTCGTCCGA-3' } \\ \text { pgl-1qfwd: } & \text { 5'-AAGTGCTTCGCCAGAAGTAGCGC-3' } \\ \text { pgl-1qrev: } & \text { 5'-GCACGAGCTTGTGGTTGTGGTG-3' } \\ \text { prg-1qfwd: } & \text { 5'-CGTGGCGTGAAGGAATGCGGTA-3' } \\ \text { prg-1qrev: } & \text { 5'-ACACACATCGGCTCTCCCAGCT-3' } \\ \text { puf-7qfwd: } & \text { 5'-GTCGAGTCGAACCGTGATGCGT-3' } \\ \text { puf-1qrev: } & \text { 5'-TTGCCAATCAAAGCGGCGGTG-3' } \\ \text { hpl-2_175qfwd: } & \text { 5'-ACGACACAGCCAGAAGCCCG-3' } \\ \text { hpl-2_175qrev: } & \text { 5'-GGCTCGGATAGCGACTGTTCACC-3' } \\ \text { lin-61qfwd: } & \text { 5'-TTTGAGCTCATCGACCCCTT-3' } \\ \text { lin-61qrev: } & \text { 5'-CCTTTGAACTCATCTGGCGG-3' }\end{array}$




\subsection{Molecular biological methods}

\subsubsection{Plasmid DNA preparation}

Isolation of plasmid DNA from $\mathrm{DH} 5 \alpha$ bacteria containing the desired plasmid was performed from a culture grown $\mathrm{O} / \mathrm{N}$ at $37^{\circ} \mathrm{C}$ in either $5 \mathrm{ml}$ or $200 \mathrm{ml}$ LB media $(1 \% \mathrm{w} / \mathrm{v}$ peptone, $0.5 \% \mathrm{w} / \mathrm{v}$ yeast extract, $0.5 \% \mathrm{w} / \mathrm{v} \mathrm{NaCl}$ ) using either nucleoBond ${ }^{\circledR}$ Xtra mini or midi kit respectively, according to the manufacturer's protocol. The isolated plasmid DNA was quantified spectrophotometrically at 260/280 nm using the NanoDrop ${ }^{\circledR}$ ND1000.

\subsubsection{DNA digestion with restriction endonucleases}

DNA was digested with various restriction endonucleases depending on the DNA sequence following either NEB or Fermentas protocols (100).

\subsubsection{Polymerase chain reaction (PCR)}

As per the requirement, appropriate plasmid templates and designed primers were used to set up the PCR as described (101). Usually, $50 \mu$ PCR mix (100 ng template DNA, $500 \mathrm{nM}$ of each chosen primer, $200 \mu \mathrm{M}$ of each dNTP, 1 unit Pfu Ultra ${ }^{\mathrm{TM}}$ II Fusion HS DNA polymerase, $5 \mu \mathrm{l}$ 10X reaction buffer provided by the manufacturer) was incubated in the PCR machine 'eppendorf mastercycler' with the following standard program: 1 min. at $94^{\circ} \mathrm{C}-\left[30 \mathrm{sec}\right.$. at $94^{\circ} \mathrm{C}-30 \mathrm{sec}$. at $55^{\circ} \mathrm{C}-30 \mathrm{sec}$. per kb of amplicon length at $\left.72^{\circ} \mathrm{C}\right] 30 \mathrm{X}-10 \mathrm{~min}$. at $72^{\circ} \mathrm{C}$.

\subsubsection{Agarose gel electrophoresis for separation and isolation of DNA fragments}

DNA fragments were separated based on their size by submarine agarose gel electrophoresis (101). DNA was mixed with 10X loading dye (10 mM EDTA, 30\% w/v glycerol, $100 \mu \mathrm{g} / \mathrm{ml}$ bromphenol blue) loaded into the wells of $0.8 \%$ to $2 \%$ agarose gels (0.8-2\% w/v agarose, $89 \mathrm{mM}$ Tris, $89 \mathrm{mM}$ boric acid, $2 \mathrm{mM}$ EDTA, 0.01\% v/v ethidium bromide, $\mathrm{pH}$ 8.0). Depending on the size of the desired DNA, the gel was run for 30-60 min. at $120 \mathrm{~V}$ in TBE buffer ( $89 \mathrm{mM}$ Tris, $89 \mathrm{mM}$ boric acid, $2 \mathrm{mM}$ EDTA, pH 8.0) using the sub-cell-GT agarose gel electrophoresis system. 
The DNA was visualized using UV light (Gel documentation system), as the fluorescence of ethidium bromide is strongly enhanced by the intercalation with DNA. In all runs, GeneRuler ${ }^{\mathrm{TM}} 1 \mathrm{~kb}$ plus DNA ladder was loaded in parallel to the samples, as size reference. If desired, single DNA fragments were isolated out of the agarose gels using the gel extraction kit according to manufacturer's instructions.

\subsubsection{Transformation of plasmids into chemically competent bacteria}

Plasmid transformation into chemically competent bacteria (2.2.6) such as $\mathrm{DH} 5 \mathrm{a}$ or BL21-CodonPlus ${ }^{\circledR}(D E 3)-(R I L)$ was performed as described (101). Chemically competent bacteria stored at $-80^{\circ} \mathrm{C}$ (in $100 \mu$ aliquots) were thawed on ice for $20 \mathrm{~min}$. Once thawed, plasmid DNA (50-500 ng) was added and bacteria were incubated for 20 min. on ice. The bacteria were heat shocked for $90 \mathrm{sec}$. at $42^{\circ} \mathrm{C}$ and cooled on ice for 5 min. $900 \mu \mathrm{l}$ of SOC medium ( $2 \% \mathrm{w} / \mathrm{v}$ tryptone, $0.5 \% \mathrm{w} / \mathrm{v}$ yeast extract, $10 \mathrm{mM} \mathrm{NaCl}, 2.5$ $\mathrm{mM} \mathrm{KCl}, 10 \mathrm{mM} \mathrm{MgCl}_{2}, 20 \mathrm{mM}$ glucose) was added to eppendorf tubes.

These tubes were incubated at $37^{\circ} \mathrm{C}$ while shaking at $800 \mathrm{rpm}$ for $60 \mathrm{~min}$. Bacteria were briefly spun at low speed (3000X g) for $1 \mathrm{~min}$. After removing the supernatant, pellet was resuspended in $50 \mu \mathrm{l} \mathrm{SOC}$ medium before plating the cells on an LB-plate $(1 \% \mathrm{w} / \mathrm{v}$ tryptone, $0.5 \%$ yeast extract, $1 \% \mathrm{w} / \mathrm{v} \mathrm{NaCl}, 1.5 \%$ agar) containing the required antibiotics $\left(100 \mu \mathrm{g} / \mathrm{ml}\right.$ ampicillin or $50 \mu \mathrm{g} / \mathrm{ml}$ kanamycin) for incubation $\mathrm{O} / \mathrm{N}$ at $37^{\circ} \mathrm{C}$.

\subsubsection{Preparation of chemically competent bacteria}

$5 \mathrm{ml}$ of LB medium was incubated with desired bacterial strain $\mathrm{O} / \mathrm{N}$ at $37^{\circ} \mathrm{C}$. The culture was transferred to $100 \mathrm{ml}$ LB medium while measuring ${ } D_{600 \mathrm{~nm}}$ every $30 \mathrm{~min}$. until it reached 0.5-0.6. At this point, the cultures were taken out of the shaker incubator, cooled on ice for $10 \mathrm{~min}$. before spinning them down at $6000 \mathrm{X} \mathrm{g}$ for $10 \mathrm{~min}$. The supernatant was removed and the pellet was resuspended in $30 \mathrm{ml}$ of TFB1 (100 mM $\mathrm{RbCl}, 50 \mathrm{mM} \mathrm{MnCl}_{2}, 30 \mathrm{mM} \mathrm{KCl}, 10 \mathrm{mM} \mathrm{CaCl}_{2}, 15 \%$ v/v glycerol, pH 5.8 (adjusted with $1 \mathrm{M}$ acetic acid)). Cells were incubated on ice for $90 \mathrm{~min}$. After incubation, the cells were spun at $6000 \mathrm{X} \mathrm{g}$ for $10 \mathrm{~min}$. The pellet was resuspended in $4 \mathrm{ml}$ of ice-cold TFB2 (10 mM MOPS, 10 mM RbCl, $75 \mathrm{mM} \mathrm{CaCl}_{2}, 15 \%$ v/v glycerol, pH 6.8 (adjusted with $1 \mathrm{M}$ $\mathrm{KOH})$ ). $100 \mu \mathrm{l}$ aliquots were prepared and stored at $-80^{\circ} \mathrm{C}$ until use. 


\subsubsection{Molecular cloning procedure}

Cloning of cDNA was performed as described (101). Primers were designed including the desired restriction sites. DNA was amplified using appropriate plasmid templates (2.2.3) and specific primers. PCR products were gel purified using gel extraction kit (2.2.4) and the amplicon was subjected to restriction digestion. Parallely, 2-5 $\mu \mathrm{g}$ of vector DNA was digested with same set of restriction enzymes (2.2.2). The restriction digested insert DNA and vector DNA were mixed in 1:6 molar ratio with $1 \mu$ T4 DNA ligase/quick ligase in $20 \mu \mathrm{l}$ reaction volume containing $5 \mu \mathrm{l} 2 \mathrm{X}$ ligase buffer. The ligation mix was incubated for $10 \mathrm{~min}$. at room temperature (RT). 2-5 $\mu$ l of ligation reaction was directly transformed into bacteria (either $\mathrm{DH} 5 \mathrm{a}$ or Nova blue). Bacteria were subsequently plated on agar plates containing appropriate antibiotic and incubated at $37^{\circ} \mathrm{C} \mathrm{O} / \mathrm{N}$. Single colonies obtained were inoculated in LB medium for plasmid preparation. After plasmid preparation from these cultures, positive clones were identified with appropriate sequencing primers at SeQLab (Göttingen, Germany).

\subsubsection{Site-directed mutagenesis}

Primer design for site-directed mutagenesis was performed using the following primer design program:

(http://www.genomics.agilent.com/primerDesignProgram.jsp?\&_requestid=304938).

A PCR (100 ng template DNA, $200 \mathrm{nM}$ of each primer, $400 \mu \mathrm{M}$ of each dNTP, 1 unit Pfu Ultra $^{T M}$ II fusion HS DNA polymerase, $5 \mu \mathrm{l} 10 \mathrm{X}$ PfU buffer) was performed with the following program: $1 \mathrm{~min}$. at $94^{\circ} \mathrm{C}-\left[30 \mathrm{sec}\right.$. at $90^{\circ} \mathrm{C}-30 \mathrm{sec}$. at $55^{\circ} \mathrm{C}-30 \mathrm{sec}$. per kb of amplicon length at $\left.72^{\circ} \mathrm{C}\right] 18 \mathrm{X}-10 \mathrm{~min}$. at $72^{\circ} \mathrm{C} .20 \mu \mathrm{l}$ of the PCR product was subjected to Dpnl digestion (10 units Dpnl, $2.2 \mu \mathrm{l}$ 10X NEB4 buffer). $5 \mu \mathrm{l}$ of Dpnl-digested DNA was directly transformed into XL1Blue ultra-competent bacteria. Bacteria were subsequently plated on agar plates containing appropriate antibiotic and incubated at $37^{\circ} \mathrm{C} \mathrm{O} / \mathrm{N}$. Single colonies obtained were inoculated in LB medium for plasmid preparation. After plasmid preparation from these cultures, positive clones were identified with appropriate sequencing primers at SeQLab. 


\subsubsection{Plasmid constructs generated in this study}

\subsubsection{1 pGEX-4T3 based hpl-2 deletion constructs}

The DNA sequence corresponding to the $h p l-2$ gene was amplified from pGEX-fulllength-hpl-2 plasmid by PCR and cloned into pGEX-4T3 plasmid via EcoRI and Xhol sites to obtain various deletion mutants (figure 3.10A) of $h p l-2$ as described (2.2.7).

\subsubsection{2 pGEX-4T3 based hpl-2 phosphomimic mutants}

To generate constructs encoding hpl-2 point mutants, we performed site-directed mutagenesis as described (2.2.8). Primers used for mutagenesis are described in table 2-12.

\subsection{Protein biochemical methods}

\subsubsection{SDS-PAGE separation of proteins}

SDS-polyacrylamide gel electrophoresis was carried out according to standard protocols $(106,107)$. Gels were casted using Mini-PROTEAN electrophoresis system. 8-15\% percent separating gels ((8-15\% acrylamide/bisacrylamide (37.1:1), $0.4 \mathrm{M}$ Tris, $0.1 \% \mathrm{w} / \mathrm{v}$ SDS, $5 \% \mathrm{v} / \mathrm{v}$ glycerol, $0.1 \% \mathrm{w} / \mathrm{v}$ APS, 0.04\% v/v TEMED, pH 8.8 (adjusted with $\mathrm{HCl}))$ and $4 \%$ stacking gels $(4 \%$ acrylamide/bisacrylamide $(37.1: 1), 0.68 \%$ Tris, $0.1 \% \mathrm{w} / \mathrm{v}$ SDS, $0.1 \% \mathrm{w} / \mathrm{v}$ APS, $0.1 \% \mathrm{v} / \mathrm{v}$ TEMED, $\mathrm{pH} 6.8$ (adjustd with $\mathrm{HCl}$ )) were used. Protein samples were resuspended in sample buffer $(62.5 \mathrm{mM}$ Tris, $8.5 \% \mathrm{v} / \mathrm{v}$ glycerol, $2 \% \mathrm{w} / \mathrm{v} \mathrm{SDS}, 100 \mathrm{\mu g} / \mathrm{ml}$ bromphenol blue, $150 \mathrm{mM}$ 2-mercaptoethanol) and boiled for $10 \mathrm{~min}$. before loading onto the gels. SDS gels were typically run at constant voltage of $150 \mathrm{~V}$ in SDS-PAGE running buffer (25 mM Tris, $200 \mathrm{mM}$ glycine, $0.1 \% \mathrm{w} / \mathrm{v}$ SDS) at RT until the blue running front reached the bottom of the gel. As size reference, $5 \mu \mathrm{l}$ of SeeBlue ${ }^{\circledR}$ plus2 pre-stained protein standard was run in parallel to the samples. 


\subsubsection{Protein detection techniques}

\subsubsection{Coomassie blue staining}

SDS-PAGE gels were submerged in coomassie staining solution $(0.05 \% \mathrm{w} / \mathrm{v}$ coomassie brilliant blue, $10 \% \mathrm{v} / \mathrm{v}$ acetic acid, $50 \% \mathrm{v} / \mathrm{v}$ methanol) for $30 \mathrm{~min}$. with gentle rocking. Gels were destained with destaining solution (10\% v/v acetic acid, $7.5 \% \mathrm{v} / \mathrm{v}$ methanol) 2 times for $60 \mathrm{~min}$. each.

\subsubsection{Western blotting}

Before western blotting, proteins were resolved using SDS-PAGE. The proteins were transferred onto nitrocellulose membranes using the mini-trans-blot system. The transfer was performed for $60 \mathrm{~min}$. at $100 \mathrm{~V}$ at $4^{\circ} \mathrm{C}$ in transfer buffer $(25 \mathrm{mM}$ Tris, 200 $\mathrm{mM}$ glycine, $20 \% \mathrm{v} / \mathrm{v}$ methanol, $0.1 \% \mathrm{w} / \mathrm{v}$ SDS). The nitrocellulose membrane was recovered by disassembling the entire system. The membrane was submerged in blocking solution made up of $5 \%$ skimmed milk powder in phosphate buffered Saline

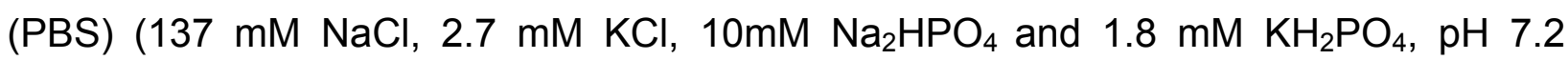
(adjusted with $\mathrm{HCl}$ )) for $60 \mathrm{~min}$. while gently rocking. Primary antibodies were diluted as per the recommendations (2.1.7) in PBS-T (PBS with $0.1 \% \mathrm{v} / \mathrm{v}$ tween ${ }^{\circledR}-20$ ) and incubated either for $1 \mathrm{hr}$. at RT for $\mathrm{O} / \mathrm{N}$ at $4^{\circ} \mathrm{C}$. The membrane was washed 4 times, 15 min. each with PBS-T to remove any unbound/non-specifically bound antibody. The membrane was incubated with secondary antibodies conjugated to horseradish peroxidase at the indicated dilution (2.1.7) for $1 \mathrm{hr}$. at RT. The membrane was again washed 4 times with PBS-T to remove unbound/non-specifically bound antibodies. The membrane was then incubated with ECL or ECL plus western blotting detection reagent and subsequently developed using gel documentation system.

\subsubsection{Expression of recombinant proteins in E. coli}

Plasmid bearing the protein-coding insert DNA was transformed into E. coli BL21CodonPlus $^{\circledR}$ (DE3)-(RIL) cells. A single colony was picked and inoculated in $100 \mathrm{ml}$ of LB media $\mathrm{O} / \mathrm{N}$ at $37^{\circ} \mathrm{C}$ in an incubator shaker (120 rpm) as a starter culture. Depending on the yield of the protein and requirement, 1-10 Itrs. of primary culture was prepared from starter culture with 1:100 ratio. The cultures were monitored until these reached 
$\mathrm{OD}_{600 \mathrm{~nm}}$ 0.5-0.7. At this point, the protein expression was induced by addition of $0.5 \mathrm{mM}$ IPTG and the cultures were further incubated for 4-7 hrs. The cultures were spun at $6000 \mathrm{Xg}$ for $15 \mathrm{~min}$. at $4^{\circ} \mathrm{C}$ and the pellet was resuspended in $10 \mathrm{ml}$ lysis buffer/ltr. The pellet containing lysis buffer was stored at $-80^{\circ} \mathrm{C}$ until further use.

\subsubsection{Purification of recombinant proteins}

The bacterial suspension in lysis buffer was thawed at $4^{\circ} \mathrm{C}$ and the cell lysis was performed using EmulsiFlex-C5 high pressure homogenizer at $4^{\circ} \mathrm{C}$ according to manufacturer's instructions. NP-40 was added to final concentration of $0.5 \%$ and the lysate was stirred for $30-60 \mathrm{~min}$. The lysate was centrifuged at $20000 \mathrm{Xg}$ for $45 \mathrm{~min}$. at $4^{\circ} \mathrm{C}$ to remove insoluble cell material. Supernatant was saved for protein purification (soluble fraction). Further purification was performed based on the type of tag of the protein of interest.

\subsubsection{Purification of 6XHis-tagged recombinant proteins}

To purify 6XHis-tagged recombinant proteins, the bacterial pellet (2.3.3) was resuspended in Ni-NTA-lysis buffer $(50 \mathrm{mM}$ sodium phosphate, $300 \mathrm{mM} \mathrm{NaCl}, 10 \mathrm{mM}$ imidazole, 2 mM PMSF, 2 mM benzamidine, $10 \mathrm{mM}$ 2-mercaptoethanol, $\mathrm{pH} \mathrm{8.0)} \mathrm{and}$ then handled as described above (2.3.4). The Ni-NTA beads were packed into a plastic gravity flow column and were equilibrated with Ni-NTA lysis buffer with 100 column volumes (CV). The supernatant obtained after clarification of the lysate was applied onto Ni-NTA column. The eluate was reloaded to allow binding of any unbound 6XHistagged protein. Once the protein was bound, column was washed with $100 \mathrm{CV}$ wash buffer (50 mM sodium phosphate, 500 mM NaCl, 10 mM imidazole, 2 mM PMSF, 2 mM benzamidine, $\mathrm{pH}$ 8.0) as per the QIAexpression manual (130). The protein bound column was never allowed to dry out during all these steps of purification. 6XHistagged protein was eluted with elution buffer $(50 \mathrm{mM}$ sodium phosphate, $100 \mathrm{mM} \mathrm{NaCl}$, 250 mM imidazole, 2 mM PMSF, 2mM benzamidine, 10 mM 2-mercaptoethanol, pH 8.0) by gravity flow. The protein eluate was collected and the protein concentration was determined spectrophotometrically using $\mathrm{OD}_{280 \mathrm{~nm}}$ for further assays. 


\subsubsection{Purification of GST-tagged proteins}

To purify GST-tagged recombinant proteins, the bacterial pellet obtained (2.3.3) was resuspended in GST lysis buffer (50 mM Tris, $150 \mathrm{mM} \mathrm{NaCl}, 1 \mathrm{mM}$ EDTA, 1mM DTT, 1 $\mathrm{mM}$ PMSF, $2 \mathrm{mM}$ benzamidine, $\mathrm{pH}$ 8) and handled as described above (2.3.4). The glutathione sepharose 4 fast flow resin was pre-equilibrated in eppendorf tubes with GST lysis buffer. The supernatant obtained after clarification of the lysate was applied to the resin. $1 \mathrm{ml}$ of resin/ttr. of culture was used for protein purification. The resin-lysate mixture was allowed to rotate on a nutator at $4^{\circ} \mathrm{C}$ for 3-4 hrs. The beads were washed 6 times with GST lysis buffer for 30 min. each at $4^{\circ} \mathrm{C}$ followed by centrifugation at $2000 \mathrm{X}$ $\mathrm{g}$ for $3 \mathrm{~min}$. GST-tagged protein was eluted with GST elution buffer for $30 \mathrm{~min}$. The protein eluate was collected and the protein concentration was determined spectrophotometrically using $\mathrm{OD}_{280 \mathrm{~nm}}$ for further assays.

\subsubsection{In-vitro translation of Myc-tagged recombinant proteins using rabbit reticulocyte lysate system}

Myc-tagged recombinant proteins were expressed using the $T_{N} T^{\circledR} T 7$ quick coupled Transcription/Translation system (Promega) according to the manufacturer's protocol.

\subsubsection{Histone peptide pull-down assay}

Histone peptide pull-down assays were performed as described (102). Avidinconjugated agarose slurry (Pierce ${ }^{\circledR}$ avidin agarose) was pre-equilibrated with PBS. $30 \mu \mathrm{l}$ of $50 \%$ slurry was added to $500 \mu \mathrm{l} \mathrm{PBS}$ along with $10 \mu \mathrm{g}$ of C-terminally biotinylated histone (either unmodified or modified) peptide. Avidin agarose-peptide mixture was incubated at RT in a thermo-mixer while shaking $(1400 \mathrm{rpm})$ for $3 \mathrm{hrs}$. Unbound peptides were removed by washing the beads 3 times with PBS. The beads were then pre-equilibrated with PD150 buffer (20 mM HEPES, $150 \mathrm{mM} \mathrm{KCl}, 0.2 \% \operatorname{triton}^{\circledR} \mathrm{X}-100$, $20 \%$ glycerol, $\mathrm{pH}$ 7.9). Purified recombinant protein $(25 \mu \mathrm{g})$ or $\mathrm{T}_{\mathrm{N}} \mathrm{T}$ prepared protein was added to the beads containing $500 \mu \mathrm{PD} 150$ buffer.

Protein-beads mixture was incubated $\mathrm{O} / \mathrm{N}$ at $4^{\circ} \mathrm{C}$. Beads were washed 6 times with PD150 buffer at $4^{\circ} \mathrm{C}$ (incubation for $10 \mathrm{~min}$. followed by centrifugation at $2000 \mathrm{X} \mathrm{g}$ ). Proteins were recovered from the beads by adding SDS-PAGE sample buffer and 
boiling for $10 \mathrm{~min}$. Proteins were resolved on SDS-PAGE gels. These gels were either stained with coomassie blue or processed for western blotting to detect the recovery of the proteins of interest.

\subsubsection{In-vitro protein pull-down}

\subsubsection{GST pull-down assay}

$20 \mu \mathrm{g}$ of GST/GST-HPL-1/GST-HPL-2 was incubated with $40 \mu \mathrm{l}$ of glutathione beads for $3 \mathrm{hrs}$. at $4^{\circ} \mathrm{C}$. The beads were washed 3 times with GST-lysis buffer $(20 \mathrm{mM}$ Tris-Cl, $200 \mathrm{mM} \mathrm{NaCl}, 1 \mathrm{mM}$ EDTA, 0.5\% NP-40, $2 \mu \mathrm{g} / \mu \mathrm{l}$ aprotinin, $1 \mu \mathrm{g} / \mu \mathrm{l}$ leupeptin, $0.7 \mu \mathrm{g} / \mathrm{ml}$ pepstatin and $25 \mu \mathrm{g} / \mathrm{ml}$ PMSF, pH 8). $45 \mu$ of $\mathrm{T}_{\mathrm{N}}$ T lysate containing either Myc-LIN-61 WT or various mutants was added to the pull-down reaction. The beads were incubated $\mathrm{O} / \mathrm{N}$ at $4^{\circ} \mathrm{C}$. Beads were washed 6 times for $5 \mathrm{~min}$. each. Bound proteins were resuspended in sample buffer and boiled for $10 \mathrm{~min}$. at $95^{\circ} \mathrm{C}$. The samples were resolved on SDS-PAGE and analyzed by western blotting.

\subsubsection{Ni-NTA pull-down assay}

$100 \mu$ of $T_{N} T$ reaction corresponding to His-LIN61 WT was added to $40 \mu \mathrm{N}$ Ni-NTA beads and incubated for $1 \mathrm{hr}$. at $4^{\circ} \mathrm{C}$. Beads were washed with Ni-NTA lysis buffer (50 $\mathrm{mM}$ sodium phosphate, $300 \mathrm{mM} \mathrm{NaCl}, 10 \mathrm{mM}$ imidazole, $2 \mathrm{mM}$ PMSF, $2 \mathrm{mM}$ benzamidine, $10 \mathrm{mM}$ 2-mercaptoethanol, pH 8.0). $2 \mu \mathrm{g}$ of GST/GST-HPL-2 was added to the beads and incubated $\mathrm{O} / \mathrm{N}$ at $4^{\circ} \mathrm{C}$. Beads were again washed 8 times $5 \mathrm{~min}$. each. Bound proteins were resuspended in sample buffer and boiled for $10 \mathrm{~min}$. at $95^{\circ} \mathrm{C}$. The samples were resolved on SDS-PAGE and analyzed by western blotting.

\subsubsection{Fluorescence anisotropy}

Flourescence Anisotrpy measurements were performed by Dr. Alexandra Stuetzer. Florescence anisotropy measurements were performed as described (219). $100 \mathrm{nM}$ of fluorescein-labeled peptide was added to microtiter well plates. To these wells, $0.4 \mu \mathrm{M}$ to $400 \mu \mathrm{M}$ of purified HPL-2 in $25 \mu \mathrm{l}$ of FP buffer $(10 \mathrm{mM}$ triethanolamine- $\mathrm{HCl}, 0.1 \mathrm{mM}$ EDTA and $150 \mathrm{mM} \mathrm{NaCl}, \mathrm{pH} 7.5)$ at $20^{\circ} \mathrm{C}$ was added. Plate chameleon multi-label counter was used to measure polarization with $485 \mathrm{~nm}$ polarization excitation filter and 
$535 \mathrm{~nm}$ emission filter in parallel and perpendicular orientation, respectively. Titration binding curves were analyzed using Kaleida-Graph (Synergy Software) as previously described (219).

\subsection{C. elegans based methods}

\subsubsection{Examination of C. elegans}

Worm strains maintained on plates were monitored using a dissecting microscope illuminated from below. For imaging, worms were prepared on 1.8\% agarose pads in 25 $\mathrm{mM}$ sodium azide. Agarose pads were generated by placing a drop of melted prewarmed agarose on a glass slide, which was immediately flattened by another glass slide placed perpendicular to the drop. This generated an extremely thin layer of agarose on which animals were placed for imaging. Images were taken using an Olympus BX60 fluorescence microscope equipped with an Olympus XC30 3.2 MP digital camera.

\subsubsection{Culturing C. elegans on plates}

C. elegans strains were grown $(70,108)$ on Nematode growth medium (NGM) plates $(0.3 \% \mathrm{w} / \mathrm{v} \mathrm{NaCl}, 0.25 \% \mathrm{w} / \mathrm{v}$ peptone, $1.7 \% \mathrm{w} / \mathrm{v}$ agar, $5 \mathrm{mg} / \mathrm{l}$ cholesterol, $1 \mathrm{mM} \mathrm{CaCl}, 1$ $\mathrm{mM} \mathrm{MgSO}_{4}, 25$ mM K-phosphate buffer ( $\mathrm{pH} \mathrm{6.0),} 100 \mu \mathrm{g} / \mathrm{ml}$ streptomycin, $10 \mu \mathrm{g} / \mathrm{ml}$ nystatin) that were seeded with E. coli OP50-1 lawn $(124,132)$. Worms were grown at various temperatures depending on the assay $\left(15-25^{\circ} \mathrm{C}\right)$. To obtain large number of worms required for biochemical experiments, super $\mathrm{NGM}$ plates $(0.3 \% \mathrm{w} / \mathrm{v} \mathrm{NaCl}, 2 \%$ w/v peptone, $1.7 \% \mathrm{w} / \mathrm{v}$ agar, $15 \mathrm{mg} / \mathrm{l}$ cholesterol, $1 \mathrm{mM} \mathrm{CaCl}_{2}, 1 \mathrm{mM} \mathrm{MgSO}_{4}, 25 \mathrm{mM} \mathrm{K}-$ phosphate buffer (pH 6.0), $100 \mu \mathrm{g} / \mathrm{ml}$ streptomycin, $10 \mu \mathrm{g} / \mathrm{ml}$ nystatin) were used. These plates can hold 10 times more worms than equivalent sized NGM plates. In order to amplify worm cultures, the worms from food-deprived/near starved plates were washed off with M9 medium (108). The worms were washed with M9 medium 3 times at 1000X g (slow break), and subsequently transferred to new super NGM plates. Alternatively, to maintain worm strains, a small chunk of agar was cut with a scalpel from the starved plate and put on a new plate until worms crawled off. To harvest the worms for 
biochemical assays, worms were washed off bacteria with M9 for 3 times and then the worm pellet was stored at $-80^{\circ} \mathrm{C}$.

\subsubsection{Culturing C. elegans in liquid medium}

Apart from super NGM plates, large quantities of worms were also obtained by growing them in liquid medium (109). 10 NGM plates with starved L1/L2 worms were harvested and inoculated in a 3 Itrs. conical flask containing $500 \mathrm{ml}$ of $\mathrm{S}$-medium $(0.1 \mathrm{M} \mathrm{NaCl}, 50$ mM K-phosphate, 5 mg/l w/v cholesterol, 10 mM K-citrate (pH 6.0), 6.4 mM EDTA, 2.5 $\mathrm{mM} \mathrm{FeSO}_{4}, 1 \mathrm{mM} \mathrm{MnCl}_{2}, 1 \mathrm{mM} \mathrm{ZnSO}_{4}, 0.1 \mathrm{mM} \mathrm{CuSO}_{4}, 3 \mathrm{mM} \mathrm{CaCl}, 3 \mathrm{mM} \mathrm{MgSO}_{4}$ ) that was supplemented with $5 \mathrm{ml}$ concentrated HB101 bacteria solution (80 times concentrated $\mathrm{O} / \mathrm{N}$ grown culture of HB101). The flask was incubated on a shaker (120 $\mathrm{rpm}$ ) at $20^{\circ} \mathrm{C}$. The growth of the worms was monitored everyday by putting a drop of medium on a glass slide under the microscope. Worms were supplemented with additional bacterial solution every alternate day. To harvest L3-staged worms, synchronized L1 worms (N2 Bristol strain) were incubated for $30 \mathrm{hrs}$. at $20^{\circ} \mathrm{C}$. To harvest adult worms, the culture was incubated at $20^{\circ} \mathrm{C}$ for $4-5$ days. The worms were harvested by transferring the culture to $50 \mathrm{ml}$ tubes, centrifuging at $1000 \mathrm{Xg}$ for $2 \mathrm{~min}$. The pellet was washed with M9 medium 3 times to remove the bacteria before the pellet was stored at $-80^{\circ} \mathrm{C}$.

\subsubsection{Bleaching of worms for synchronization ordecontamination}

Gravid adult worms were washed 2-3 times with water and treated with a bleach solution $(0.5 \mathrm{M} \mathrm{NaOH}, 1.2 \% \mathrm{NaClO})$ (108). The worms in bleach solution were vigorously shaken for $10 \mathrm{~min}$. while vortexing constantly. The pellet was washed with M9 medium 3 times (centrifugation at 1000X $\mathrm{g}$ for $3 \mathrm{~min}$. with a slow break) before the embryos were placed on a new plate to obtain decontaminated worms. If synchronization is desired, embryos were resuspended in M9 medium and allowed to hatch $\mathrm{O} / \mathrm{N}$ before they get arrested at $\mathrm{L} 1$ stage. Synchronized $\mathrm{L} 1$ worms were transferred to new plates. 


\subsubsection{Freezing and recovery of C. elegans stocks}

The worms grown on NGM plates and just starved at L1-L2 stage were chosen for freezing as L1 worms have maximum chance of survival after freeze-thawing procedure. 2-3 such plates of worms were washed with water and allowed to settle down. After 3 washes, water was decanted off leaving behind $1 \mathrm{ml}$. An equal volume of freezing solution $\left(0.3 \% \mathrm{w} / \mathrm{v} \mathrm{KH}_{2} \mathrm{PO}_{4}, 0.6 \% \mathrm{w} / \mathrm{v} \mathrm{Na} \mathrm{HPO}_{4}, 0.5 \% \mathrm{w} / \mathrm{v} \mathrm{NaCl}, 1 \mathrm{mM} \mathrm{MgSO}\right.$, $30 \% \mathrm{v} / \mathrm{v}$ glycerol) was added $(124,132)$. The worm suspension was added to cryotubes $(1-2 \mathrm{ml})$ and shaken vigorously. The cryotubes were placed horizontally in a styrofoam box to ensure slow cooling and also avoid settling down of worms. These cryotubes were kept at $-80^{\circ} \mathrm{C}$. For thawing worm stocks, a sterile toothpick or $200 \mu$ eppendorf tip was inserted in a cryotube and the solidified worm suspension was placed on an agar plate. Worms were then allowed to recover at $20^{\circ} \mathrm{C}$ for $4-6 \mathrm{hrs}$.

\subsubsection{Single worm PCR}

Single worm PCR is a genotyping method similar to tail DNA isolation in mice. A single hermaphrodite was picked into a PCR tube with $5 \mu \mathrm{l}$ single worm lysis buffer (10 mM Tris, $50 \mathrm{mM} \mathrm{KCl}, 2.5 \mathrm{mM} \mathrm{MgCl}_{2}, 0.45 \% \mathrm{NP}-40,0.45 \%$ tween $20,0.01 \%$ gelatin, 100 $\mu \mathrm{g} / \mathrm{ml}$ proteinase- $\mathrm{K}, \mathrm{pH} 8.3$ ) and frozen at $-80^{\circ} \mathrm{C}$ for $30 \mathrm{~min}$. to freeze-crack the worm. The worm lysate was incubated at $60^{\circ} \mathrm{C}$ for $60 \mathrm{~min}$. followed by $95^{\circ} \mathrm{C}$ for $15 \mathrm{~min}$. (for proteinase-K activity). Next, a PCR was performed using three pairs of primers that distinguish WT allele and mutant allele, based on the size of the PCR product. This allows identification of genotypes of a parent worm for the gene of interest against which the primers were designed. For PCR, $2.5 \mu \mathrm{l}$ of worm lysate was added to $22.5 \mu \mathrm{l}$ of worm PCR mix $\left(2.2 \mathrm{mM} \mathrm{MgCl}_{2}, 222 \mu \mathrm{M}\right.$ of each dNTP, $0.7 \mu \mathrm{M}$ of each chosen primer, 0.5 unit/22.5 $\mu \mathrm{l}$ Taq polymerase (Fermentas)). PCR was performed at the following conditions: $1 \mathrm{~min}$. at $94^{\circ} \mathrm{C}-\left[30 \mathrm{sec}\right.$. at $94^{\circ} \mathrm{C}-30 \mathrm{sec}$. at $57^{\circ} \mathrm{C}-40 \mathrm{sec}$. at $72^{\circ} \mathrm{C}$ ] $40 \mathrm{X}-5 \mathrm{~min}$. at $72^{\circ} \mathrm{C}$. When more than one single worm PCR had to be performed per worm; the worm was picked into $5 \mu \mathrm{l}$ (for two PCRs) or $7.5 \mu \mathrm{l}$ (for three PCRs) lysis buffer. The sample was separated into $2.5 \mu$ fractions for the different PCRs. 


\subsubsection{C. elegans crosses}

In C. elegans, natural frequency of males is about $\sim 0.1 \%$ (arise by spontaneous $\mathrm{X}$ chromosome non-dysjunction). The progeny obtained by cross-fertilization consists of $\sim 50 \%$ males, whereas the progeny from self-fertilization is $\sim 0.1 \%$. This requires the male population to be maintained by crossing. $C$. elegans male frequency can be increased by giving heat shock to $L 4$ worms at $32^{\circ} \mathrm{C}$ for $4 \mathrm{hrs}$. We obtained males using this method. To start a cross, 15-20 males of one strain were put together with 6-8 young adult hermaphrodites of another strain. Worms were allowed to mate for 2-3 days. Males were then removed from the plate. F1 progeny were singled out and allowed to lay eggs. F1 progeny were subjected to single worm PCR and scored for heterozygotes. Heterozygous F1 progeny were allowed to lay eggs and F2 progeny were singled out and allowed to lay eggs. The F2 progeny were then scored for the desired double mutant using single worm PCR.

\subsubsection{C. elegans protein extract preparation}

C. elegans protein extract was prepared with some modifications according to Wysocka et al. (110). For extract preparations, $10-15 \mathrm{~g}$ of frozen $C$. elegans, derived from liquid $C$. elegans cultures or super NGM plates were grounded using a pre-chilled mortar and pestle in liquid $\mathrm{N}_{2}$.

\subsubsection{C. elegans nuclear extract preparation}

The worm powder was resuspended in an equal volume of $2 \mathrm{X}$ buffer $\mathrm{A}$ (10 mM HEPES, $30 \mathrm{mM} \mathrm{MgCl}$, $20 \mathrm{mM} \mathrm{KCl}, 1 \mathrm{mM}$ DTT, 1X EDTA-free complete protease inhibitor cocktail, $\mathrm{pH}$ 7.9) before the powder was thawed. The worms were lysed using the bioruptor for $30 \mathrm{~min}$. (30 sec. ON, $30 \mathrm{sec}$. OFF). The lysate was cleared by centrifugation at $18000 \mathrm{Xg}$ at $4^{\circ} \mathrm{C}$ for $30 \mathrm{~min}$. The supernatant-I was stored at $4^{\circ} \mathrm{C}$ while the pellet was resuspended in high salt buffer C (20 mM HEPES, $420 \mathrm{mM} \mathrm{KCl,} 1.5 \mathrm{mM}$ $\mathrm{MgCl}_{2}, 0.2 \mathrm{mM}$ EDTA, 25\% v/v glycerol, 1X EDTA-free complete protease inhibitor cocktail, $\mathrm{pH} 7.4$ ) and incubated for $2 \mathrm{hrs}$. at $4^{\circ} \mathrm{C}$ in a thermo-mixer at $1400 \mathrm{rpm}$. The lysate was centrifuged at $18000 \mathrm{Xg}$ at $4^{\circ} \mathrm{C}$ for $20 \mathrm{~min}$. The supernatant-Il was pooled with supernatant-I and dialyzed against buffer D (20 mM HEPES, $150 \mathrm{mM} \mathrm{KCl,} 0.2 \mathrm{mM}$ 
EDTA, $0.2 \% \mathrm{v} / \mathrm{v}$ triton-X100, 20\% v/v glycerol, $1 \mathrm{mM}$ DTT, $2 \mathrm{mM}$ PMSF, $2 \mathrm{mM}$ benzamidine, $\mathrm{pH}$ 7.9). Total protein concentration was determined by micro BCA assay kit.

\subsubsection{Generation of crude C. elegans lysate for western blot analysis}

50-100 adult worms were transferred to a $1.5 \mathrm{ml}$ tube and washed with M9 medium. For homogenization, an equal amount of nematode solubilization buffer $(0.3 \% \mathrm{v} / \mathrm{v}$ ethanolamine, $2 \mathrm{mM}$ EDTA, $1 \mathrm{mM}$ PMSF, $5 \mathrm{mM}$ DTT, $\mathrm{pH}$ 8) was added (111). The sample was sonicated in the bioruptor for $30 \mathrm{~min}$. (30 sec. ON; $30 \mathrm{sec}$. OFF). Afterwards, an equal volume of $2 X$ SDS-PAGE sample buffer was added and the sample was boiled for $10 \mathrm{~min}$. After clarification (centrifugation for $10 \mathrm{~min}$. at $16000 \mathrm{Xg}$ at $\left.4^{\circ} \mathrm{C}\right), 15-20 \mu \mathrm{l}$ of the supernatant was loaded onto SDS-PAGE gel, and processed for western blotting.

\subsubsection{Histone peptide pull-down assay using C. elegans extract}

Histone peptide pull-down assays were conducted as described with the following modification (110). $1 \mathrm{ml}(10 \mathrm{mg} / \mathrm{ml})$ of $C$. elegans extract was used instead of recombinant protein.

\subsubsection{Competition peptide pull-down assay}

C. elegans extract was prepared as described (2.4.8.1). Recombinant GST-CSD protein was added in increasing amounts to the extract and incubated for $1 \mathrm{hr}$. at $4^{\circ} \mathrm{C}$. The extract containing recombinant protein was used to perform peptide pull-down as described in (2.4.9).

\subsubsection{Immunoprecipitation from C. elegans extract}

\subsection{1/mmunoprecipitation of endogenous proteins from C. elegans extract}

Immunoprecipitation was performed using either rabbit anti-LIN-61, rabbit anti-HPL-2 or rabbit anti-LIN-13 antibodies and $C$. elegans nuclear extracts. Antibodies were crosslinked to dynabeads M-280 sheep anti-rabbit IgG as per the manufacturer's instructions. 
Either $20 \mu \mathrm{l}$ of anti-LIN-61 or $5 \mu \mathrm{l}$ of anti-HPL-2/anti-LIN-13 antibodies were incubated with $50 \mu \mathrm{l}$ beads $\mathrm{O} / \mathrm{N}$ at $4^{\circ} \mathrm{C}$ on a nutator. Unbound antibodies were removed by washing the beads with PBS 3 times. The beads were resuspended in $0.2 \mathrm{M}$ triethanolamine $(\mathrm{pH}$ 8.2). Beads were incubated with $1 \mathrm{ml} 20 \mathrm{mM}$ DMP in $0.2 \mathrm{M}$ triethanolamine at RT on a nutator for $60 \mathrm{~min}$. The cross-linking was quenched by

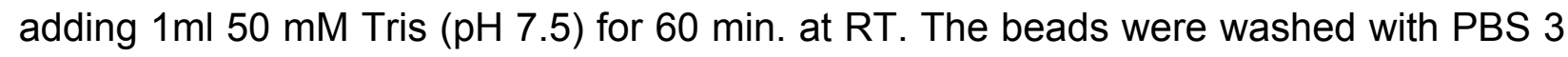
times before they were used for immunoprecipitation. $5 \mathrm{mg}$ of total protein extract was incubated with beads for $30 \mathrm{~min}$. at RT. Subsequently; the beads were washed 6 times with buffer $\mathrm{D}$ (addition of $1 \mathrm{ml}$ buffer $\mathrm{D}$ followed by immunoprecipitation incubation for 5 min. followed by centrifuge at $2000 \mathrm{Xg}$ for $1 \mathrm{~min}$.). The bound proteins were dissolved in sample buffer and boiled for $10 \mathrm{~min}$. at $95^{\circ} \mathrm{C}$. Proteins were resolved on SDS-PAGE, transferred onto nitrocellulose membrane and detected using various antibodies.

\subsubsection{GFP immunoprecipitation from C. elegans nuclear extract}

For immunoprecipitation of GFP from nuclear extracts, $10 \mu \mathrm{l}$ of GFP_Trap was incubated with $5 \mathrm{mg}$ of nuclear extract $\mathrm{O} / \mathrm{N}$ at $4^{\circ} \mathrm{C}$. The beads were washed 6 times with buffer $D$ (addition of $1 \mathrm{ml}$ buffer $D$ followed by incubation for $5 \mathrm{~min}$. followed by centrifuge at $2000 \mathrm{Xg}$ for $1 \mathrm{~min}$.). The bound proteins were dissolved in sample buffer and boiled for $10 \mathrm{~min}$ at $95^{\circ} \mathrm{C}$. Proteins were resolved on SDS-PAGE, transferred onto nitrocellulose membrane and detected using various antibodies.

\subsubsection{Competitive depletion assay}

For competitive depletion assays, $100 \mu \mathrm{g}$ of GST-HPL-2 was immobilized to $30 \mu \mathrm{l}$ glutathione beads in buffer D (20 mM HEPES, $150 \mathrm{mM} \mathrm{KCl}, 0.2 \mathrm{mM}$ EDTA, 0.2\% v/v triton-X100, 20\% v/v glycerol, 1 mM DTT, 2 mM PMSF, 2 mM benzamidine, pH 7.9) at $4^{\circ} \mathrm{C}$ for $3 \mathrm{hrs}$.

The beads were washed 3 times with buffer D. $1 \mathrm{ml}$ of $10 \mathrm{mg} / \mathrm{ml}$ lin-61 nuclear extract was added to the glutathione beads and incubated at $4^{\circ} \mathrm{C}$ for $2 \mathrm{hrs}$. The unbound fraction was collected and further processed for peptide pull-down assay (2.4.9). For another round of depletion, the unbound fraction was added to the new GST-HPL-2 conjugated glutathione beads and incubated as above. 


\subsubsection{C. elegans RNA isolation}

RNA isolation was performed as described (112). $100 \mu \mathrm{l}$ of worm pellet was resuspended in TRI-REAGENT ${ }^{\mathrm{TM}}$ and freeze-thawed 3 times to break the cuticle of worms. After final thawing at RT, $200 \mu \mathrm{l}$ of Chloroform:Isoamyl alcohol (24:1) was added per $\mathrm{ml}$ of TRI-REAGENT ${ }^{\mathrm{TM}}$. The samples were vigorously shaken and incubated at RT for $3 \mathrm{~min}$. Samples were centrifuged at $12000 \mathrm{Xg}$ for $15 \mathrm{~min}$. at $4^{\circ} \mathrm{C}$. The aqueous phase was transferred to new tubes and an equal volume of isopropanol was added to precipitate the RNA. Samples were incubated at RT for $10 \mathrm{~min}$. before centrifugation at $12000 \mathrm{X} \mathrm{g}$ for $10 \mathrm{~min}$. at $4^{\circ} \mathrm{C}$. The RNA pellet was washed with $75 \%$ ethanol and spun at 7500X $\mathrm{g}$ for $10 \mathrm{~min}$. The resulting pellet was air dried (briefly) and resuspended in RNase-free water. RNA was quantified spectrophotometrically.

To remove genomic DNA contamination, total RNA was treated with recombinant DNasel according to manufacturer's instructions. Briefly, 0.1 units $/ \mu l$ of DNasel, $1 \mathrm{X}$ DNasel buffer (400 mM Tris- $\mathrm{HCl}, 80 \mathrm{mM} \mathrm{MgCl} 2,50 \mathrm{mM}$ DTT, pH 7.5), $5 \mu \mathrm{g}$ total RNA, 0.8 units/ $\mu \mathrm{R}$ RNaseOUT ${ }^{\mathrm{TM}}$ were combined in $50 \mu \mathrm{l}$ reaction mix and incubated for 30 min. at $37^{\circ} \mathrm{C}$. To inactivate the DNasel and purify RNA, phenol/chloroform extraction was performed. The aqueous phase was combined with 0.1 volumes of $3 \mathrm{M}$ Sodium acetate, 2.5 volumes of cold $100 \%$ ethanol to precipitate the RNA. The samples were incubated at $-80^{\circ} \mathrm{C}$ for $30 \mathrm{~min}$. and centrifuged at $12000 \mathrm{Xg}$ for $15 \mathrm{~min}$. at $4^{\circ} \mathrm{C}$. The RNA pellet was washed with $70 \%$ ethanol and spun down at $8000 \mathrm{Xg}$ for $5 \mathrm{~min}$. at $4^{\circ} \mathrm{C}$. The resulting pellet was air-dried and resuspended in RNase-free water and measured spectrophotometrically. 


\subsubsection{Microarray analysis}

Microarray analysis was performed by Lena Hyatt and Lennart Opitz. $200 \mathrm{ng}$ of total RNA was used as a starting material. RNA amplification and labeling was performed using low input quick amp labeling kit, according to manufacturer's instructions. It uses T7 RNA polymerase to simultaneously amplify RNA as well as incorporate cyanine-3labeled CTP for detection. Amplified RNA was purified using RNeasy Mini kit, according to manufacturer's instructions. Hybridization to the C. elegans Agilent array was carried out using the Agilent gene expression hybridization kit. Lennart Opitz at Transcriptome facility (University of Göttingen) performed data analysis and quality control.

\subsubsection{RNA-seq analysis}

RNA-seq analysis was performed in collaboration with Dr. Stefan Bonn and Dr. Vincenzo Capace (ENI Göttingen, Germany). All libraries were prepared with Truseq RNA library preparation kit $\mathrm{v} 2$ and run on an illumina HiSeq2000 ${ }^{\mathrm{TM}}$ (50 bp single end sequencing). Raw data was mapped to the $C$. elegans genome (WBCel235/ce10) using STAR 2.3.0e_r291 (113) with the following options:

--outFilterMismatchNmax 2: number of mismatches permitted(2);

--sjdbOverhang 49: lenght of read - 1;

then after computing the basic quality control with samtools-0.1.18 and FastQC V0.10.1 (http://www.bioinformatics.babraham.ac.uk/projects/fastqc/). The uniquely mapped reads are counted with HTSeq-count 0.6.0. The differential expression analysis was performed by using DESeq2_1.6.3 (114).

\subsubsection{6 cDNA synthesis and real time RT-PCR}

For cDNA synthesis, $100 \mathrm{ng}$ of total RNA was combined with $0.25 \mu \mathrm{M}$ random hexamers and $0.5 \mathrm{mM} d N T P s$ and incubated at $65^{\circ} \mathrm{C}$ for $5 \mathrm{~min}$. Once the reaction was cooled, 1X RT buffer (250 mM Tris- $\mathrm{HCl}, 375 \mathrm{mM} \mathrm{KCl}, 15 \mathrm{mM} \mathrm{MgCl}$, $50 \mathrm{mM} \mathrm{DTT}$, pH 7.5) and 5 units/ $\mu$ l of maxima $\mathrm{H}$ minus reverse transcriptase were added to the reaction mixture of $20 \mu \mathrm{l}$. The reaction was incubated at $25^{\circ} \mathrm{C}, 50^{\circ} \mathrm{C}$ and $85^{\circ} \mathrm{C}$ for $10 \mathrm{~min}$., 15 min., and 5 min. respectively. The cDNA was stored at $-20^{\circ} \mathrm{C}$.

Real time RT-PCR was performed using $1 \mu \mathrm{l}$ of $\mathrm{cDNA}, 0.4 \mu \mathrm{M}$ forward and reverse 
primers, $1 \mathrm{XiQ}^{\mathrm{TM}} \mathrm{SYBR}^{\circledR}$ green supermix in a final volume of $20 \mu$ l. Cycling conditions were: $95^{\circ} \mathrm{C}-15$ min., $\left[95^{\circ} \mathrm{C}-15\right.$ sec., $55^{\circ} \mathrm{C}-20 \mathrm{sec}$., $72^{\circ} \mathrm{C}-30 \mathrm{sec}$, read plate] X40 followed by melting curve analysis. For normalization of the data, housekeeping gene rpl-26 was used. The fold difference was calculated using the following equation:

$$
\begin{gathered}
\Delta \Delta \mathrm{C}_{\mathrm{t}}=\mathrm{C}_{\mathrm{t} \text { goi }}-\mathrm{C}_{\mathrm{t} \text { ref }} \\
\text { Linear fold change }=-2^{\Delta \Delta \mathrm{Ct}}
\end{gathered}
$$

where, $\mathrm{C}_{\mathrm{t}}$ : cycle threshold; goi: gene of interest; ref: reference gene.

The primers used for real time RT-PCR analysis are described in table 2-12.

\subsubsection{RNAi feeding assay}

RNAi feeding assays were performed together with the MSc student Elizabeth Miller. RNAi feeding assays were performed as described (115). RNAi bacterial clones were obtained from the Ahringer library (Source Bioscience). Freshly transformed HT115 bacteria were grown for not more than 10-12 hrs. in LB+100 $\mu \mathrm{g} / \mathrm{mL}$ ampicillin, spread on RNAi plates (NGM + $25 \mu \mathrm{g} / \mathrm{mL}$ carbenicillin + $1 \mathrm{mM} \mathrm{IPTG)} \mathrm{and} \mathrm{incubated} \mathrm{at} \mathrm{RT} \mathrm{for} 24$ hrs. Embryos were placed on RNAi plates and incubated at $20^{\circ} \mathrm{C}$ until the desired developmental stage was reached. For all knock-down experiments worms were grown for two generations on RNAi plates. Second generation L3 worms were collected for RNA isolation/protein extraction.

\subsubsection{Brood size assay}

Brood size assays were performed together with the MSc student, Elizabeth Miller. RNAi assays were performed as described (2.4.17), and second generation L3 worms were plated individually on NGM agar plates seeded with E. coli, OP50. The total number of eggs hatched was counted over a period of 4-5 days.

\subsubsection{SynMuv analysis}

SynMuv analysis was performed as described (97). Worms were scored as SynMuv if they had more than two vulval projections. 


\subsection{Bioinformatics data analysis pipeline}

\subsubsection{ChIP-seq analysis pipeline}

ChIP-seq analysis was performed in collaboration with $\mathrm{Dr}$. Stefan Bonn and Orr Shomroni (ENI Göttingen, Germany). We downloaded the raw datasets for all ChIP-seq factors from the modENCODE server. The data was fed to MACS2 peak finding function (MACS2 (https://github.com/taoliu/MACS/). All the peaks that were found across replicate samples were overlapped with a percentage overlap of $50 \%$ using multi-intersectBED function from BEDTOOLS (http://bedtools.readthedocs.org/en/latest/index.html). These peaks were then annotated using HOMER function for genomic features (promoter, intron, exon, TSS, TTS, intergenic etc. http://homer.salk.edu/homer/chipseq/annotation.html).

Table 2.13: ChIP-seq datasets selected from modENCODE for analysis

\begin{tabular}{|c|c|c|c|c|c|}
\hline $\begin{array}{c}\text { Dataset } \\
\text { no. }\end{array}$ & $\begin{array}{c}\text { Transcription } \\
\text { factor/ } \\
\text { Modification }\end{array}$ & $\begin{array}{c}\text { Array } \\
\text { / seq. } \\
\text { type }\end{array}$ & $\begin{array}{c}\text { ModENCODE } \\
\text { ID }\end{array}$ & Antibody & Conditions \\
\hline 1 & HPL-2 & $\begin{array}{c}\text { ChIP } \\
-\end{array}$ & 5976 & $\begin{array}{c}\text { SDQ2340 } \\
\text { (Novus) }\end{array}$ & $\begin{array}{c}\text { N2, L3 } \\
\text { stage, 20 } \\
\text { C }\end{array}$ \\
\hline 2 & LIN-61 & $\begin{array}{c}\text { ChIP } \\
-\end{array}$ & 5980 & $\begin{array}{c}\text { HM4077 } \\
\text { (Prof. } \\
\text { Horvitz) }\end{array}$ & $\begin{array}{c}\text { N2, L3 } \\
\text { stage, 20 } \\
\text { C }\end{array}$ \\
\hline 3 & H3K9me3 & $\begin{array}{c}\text { ChIP } \\
-\end{array}$ & 5040 & $\begin{array}{l}\text { AB8898 } \\
\text { H3K9me3 } \\
: 339901\end{array}$ & $\begin{array}{c}\text { N2, L3 } \\
\text { stage, 20 } \\
\text { C }\end{array}$ \\
\hline 4 & LIN-13 & ChIP & 4795 & $\begin{array}{c}\text { Hymen } \\
\text { lab GFP } \\
\text { antibody }\end{array}$ & $\begin{array}{c}\text { OP49, L4 } \\
\text { stage, 20 } \\
\text { C }\end{array}$ \\
\hline
\end{tabular}




\section{Results}

\subsection{HPL-2 - H3K9me3 interaction in C. elegans}

Based on the knowledge on mammalian HP1 proteins, we proposed that H3K9me3 might serve as a platform for the recruitment of HPL-2 binding to chromatin in C. elegans. We therefore started with biochemical analysis HPL-2-H3K9me3 interaction.

\subsubsection{HPL-2 associates with H3K9me3 only in the presence of LIN-61}

We started with $\mathrm{H} 3 \mathrm{~K} 9 \mathrm{me} 3$ peptide pull-down assay using C. elegans extract. We tested whether HPL-2 from the extract has preferential binding to H3K9me3. LIN-61, a MBT protein has been reported to bind H3K9me3 in C. elegans (97). Our peptide pull-down assay enriched both HPL-2 as well as LIN-61 on H3K9me3 peptide (figure 3.1).

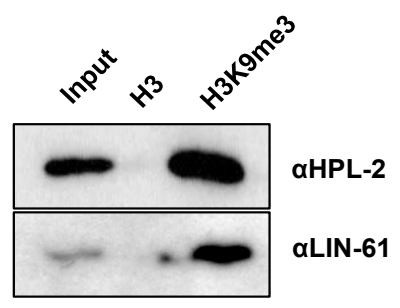

Figure 3.1: Verification of HPL-2 as $\mathrm{H} 3 \mathrm{~K} 9 \mathrm{me} 3$ binding protein

Peptide pull-down assay using either $\mathrm{H} 3$ unmodified or $\mathrm{H} 3 \mathrm{~K} 9 \mathrm{me} 3$ peptide was performed with $\mathrm{N} 2$ extract. Bound proteins were resuspended in sample buffer, resolved on SDS-PAGE and analyzed by western blotting using anti-HPL-2 (top panel) and antiLIN-61 (bottom panel) antibodies.

It has been reported that $h p l-2$ and lin-61 genetically interact during vulva development (97). Therefore, we hypothesized that both HPL-2 and LIN-61 associate with H3K9me3 independently to regulate vulva development. In order to test this, we set up peptide pull-down assays using N2, hpl-2 and lin-61 worm nuclear extracts. We analyzed the binding of HPL-2 in lin-61 mutant worm extracts as well as the binding of LIN-61 in hpl-2 mutant worm extracts to $\mathrm{H} 3 \mathrm{~K} 9 \mathrm{me} 3$ peptide. 


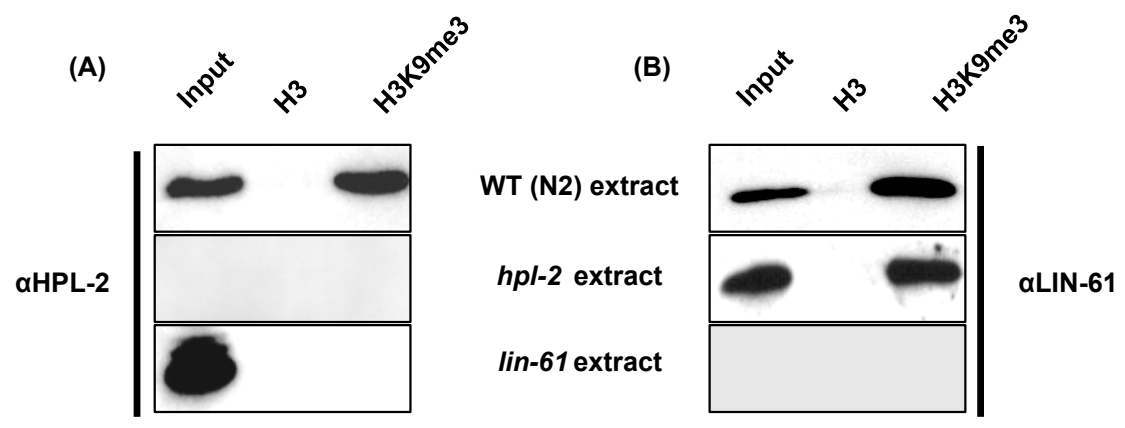

\section{Figure 3.2: HPL-2 binding to $\mathrm{H} 3 \mathrm{~K} 9 \mathrm{me} 3$ is lost in lin-61 mutant extracts}

Peptide pull-down assay in N2 (top lane), hpl-2 (middle lane) and lin-61 (bottom lane) worm extracts using either $\mathrm{H} 3$ unmodified or H3K9me3 peptide. Bound proteins were resuspended in sample buffer, resolved on SDS-PAGE and analyzed by western blotting using (A) anti-HPL-2 or (B) anti-LIN-61 antibodies.

We found that LIN-61 binding to the H3K9me3 peptide in hpl-2 mutant worm extracts remained unaffected whereas HPL-2 binding to H3K9me3 peptide in lin-61 mutant worm extracts was abolished (figure 3.2). These findings were unexpected, as all the analyzed HP1 family proteins so far have been shown to directly bind H3K9me3(123).

\subsubsection{HPL-2 directly binds H3K9me3 in vitro}

We found that HPL-2 failed to bind the H3K9me3 peptide in the absence of LIN-61. We asked whether HPL-2 inherently lacks the ability to recognize H3K9me3. We therefore, tested the binding of HPL-2 to the H3K9me3 peptide in a recombinant system. We performed peptide pull-down assays using recombinant GST-HPL-2 and found that HPL-2 binds to the H3K9me3 peptide compared to the corresponding unmodified $\mathrm{H} 3$ peptide (figure 3.3). In addition to using GST protein as a negative control, we also used GST-HPL-1 as another control. We found that HPL-1 also has the ability to bind H3K9me3 peptide in vitro (figure 3.3). This was an expected result since the amino acids in HP1 proteins involved in H3K9me3 interactions are conserved in both HPL-2 and HPL-1 (figure 3.4). 


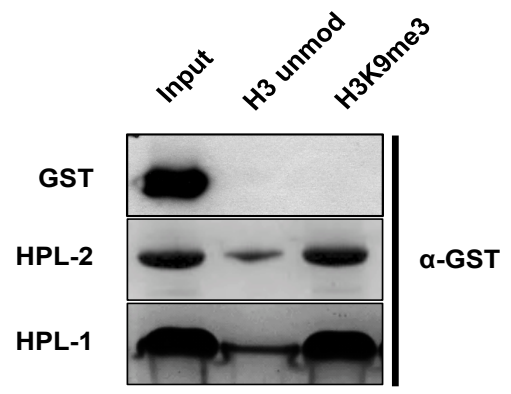

\section{Figure 3.3: Recombinant HPL-2 binds H3K9me3}

Peptide pull-down assay was performed using either recombinant GST-HPL-2, GSTHPL-1 or GST alone and either H3 unmodified or H3K9me3 peptide. Bound protein was resuspended in sample buffer, resolved on SDS-PAGE and analyzed by western blotting using anti-GST antibody.

Next, in order to quantify the interaction of HPL-2 with H3K9me3, we performed fluorescence polarization binding assays. We found that HPL-2 binds to the H3K9me3 peptide almost 100 times stronger compared to the unmodified $\mathrm{H} 3$ peptide. The apparent dissociation constants obtained from the binding assay were, $K_{D}=2.14 \pm 0.19$ $\mu \mathrm{M}$ for $\mathrm{H} 3 \mathrm{~K} 9 \mathrm{me} 3$ as opposed to $\mathrm{K}_{\mathrm{D}}=240.19 \pm 16 \mu \mathrm{M}$ for unmodified H3 peptide. HPL-2 also bound to H3K9me1 (10 times stronger) and H3K9me2 (16 times stronger) with intermediate strengths over unmodified H3 peptide (figure 3.5). 


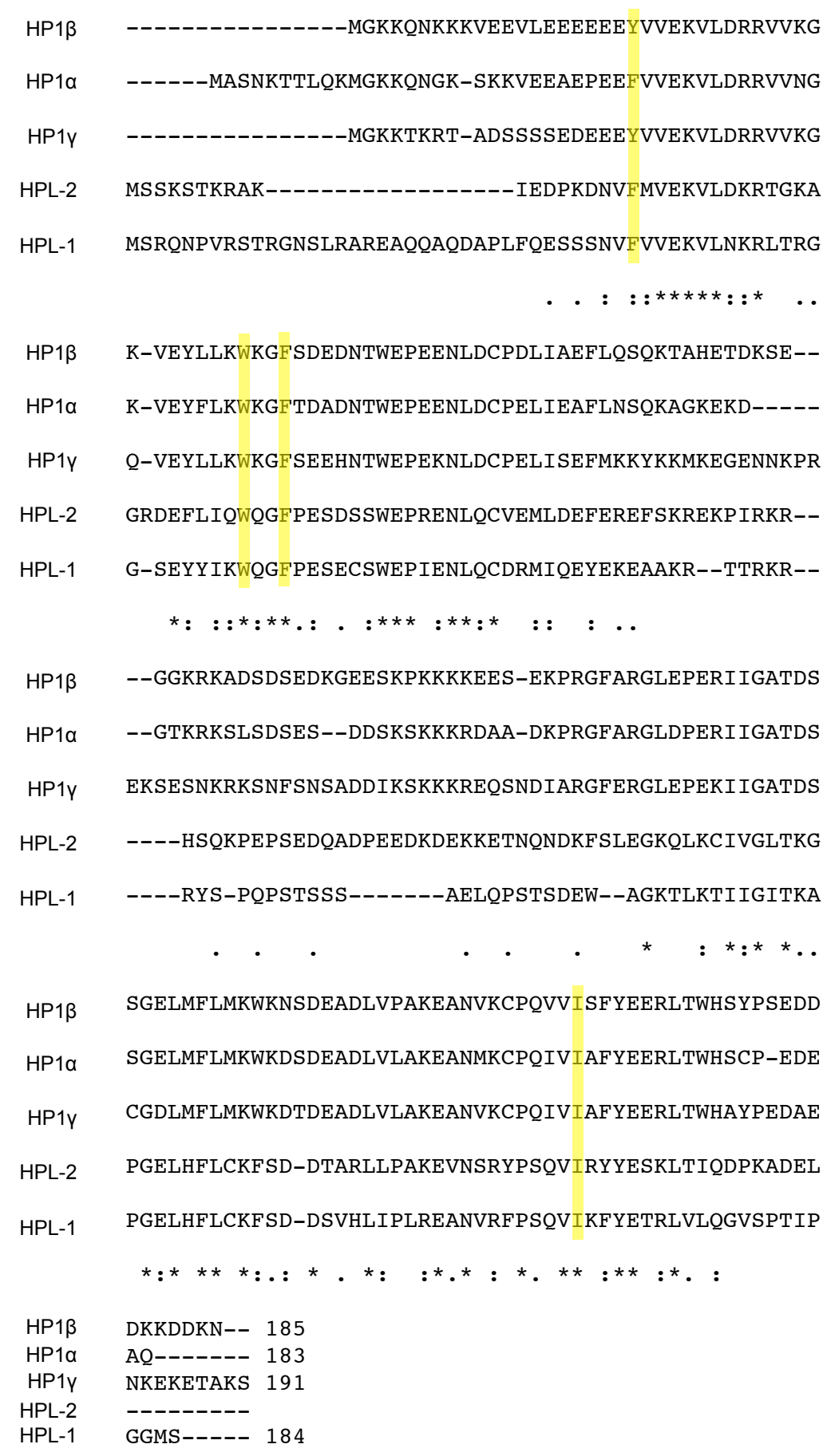

Figure 3.4: HPL-2 in C. elegans is a bonafide HP1 protein

Multiple sequence alignment of HPL-1 and HPL-2 with mammalian HP1 isoforms

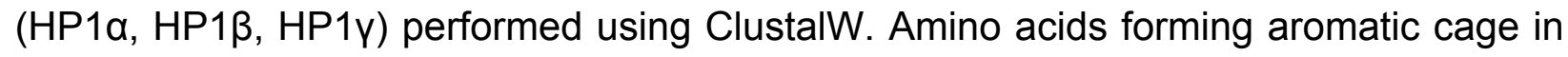
the CD as well as conserved HP1 $\beta 1161$ are highlighted. 


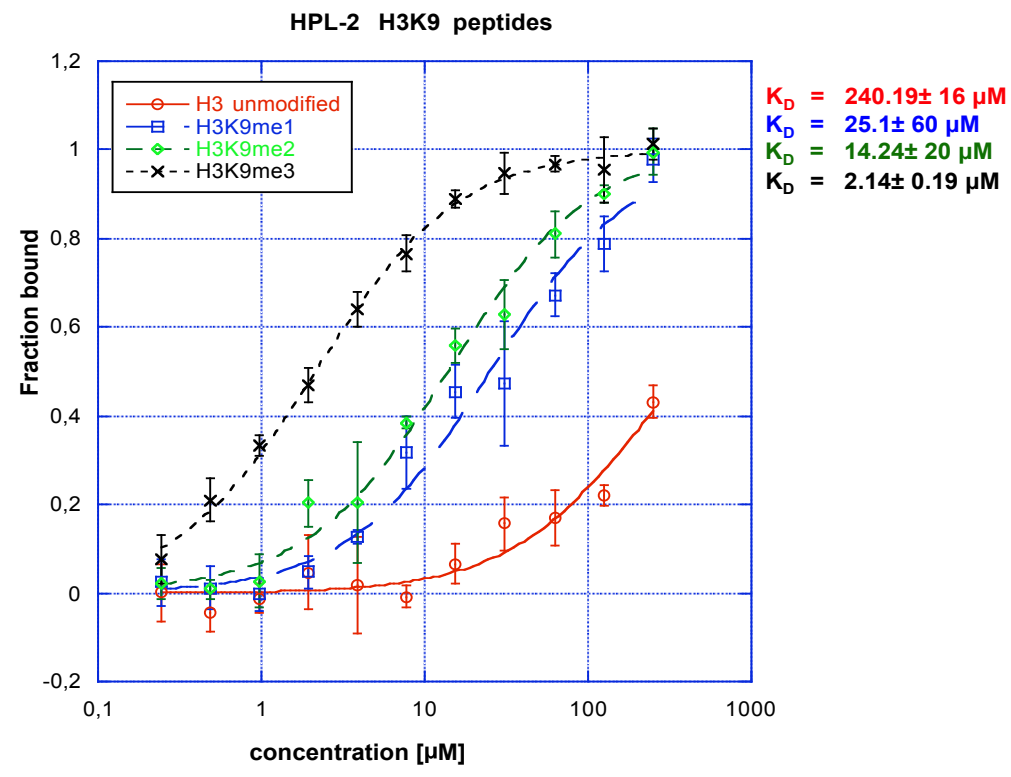

Figure 3.5: Recombinant HPL-2 binds H3K9me peptides

Fluorescence polarization measurements using fluorescein labeled H3 unmodified, H3K9me1, H3K9me2, H3K9me3 and recombinant HPL-2. Average bound fraction of H3 peptides from three measurements is plotted. $K_{D}$ values of interaction of recombinant HPL-2 with the indicated peptides as measured by fluorescence polarization binding assays are given on the right. Average of at least three experiments including standard deviation is given. FP measurements were performed by Dr. Alexandra Stuetzer.

We concluded that in worm extracts, binding of HPL-2 to the H3K9me3 peptide depends on LIN-61, whereas HPL-2 directly binds H3K9me3 peptide in vitro.

\subsubsection{HPL-2 binding to H3K9me3 is mediated by LIN-61}

We argued that the loss of HPL-2 binding to $\mathrm{H} 3 \mathrm{~K} 9$ me 3 peptide in lin-61 mutant extract is an artifact of the specific lin-61 mutant that we used in our peptide pull-down assays or an indirect effect caused by loss of LIN-61 protein.

We designed an assay, where we supplemented the lin-61 extract with recombinant LIN- 61 protein. We hypothesized that, if HPL-2 truly depends on LIN-61 for its binding to H3K9me3 peptide, recombinantly added LIN-61 should recover HPL-2 on H3K9me3 peptide.

We expressed LIN-61 protein using in-vitro translation rabbit reticulocyte system $\left(\mathrm{T}_{\mathrm{N}} \mathrm{T}\right.$ 
mix). We found that addition of $T_{N} T$ mix containing WT-LIN-61 protein recovers HPL-2 on H3K9me3 peptide, whereas control $\mathrm{T}_{N} T$ mix did not recover HPL-2. LIN-61 binds to H3K9me3 via an aromatic cage formed by three aromatic amino acid side chains (97). When we supplemented MUT-LIN-61 (aromatic amino acids replaced to alanine), we did not observe the recovery of HPL-2. This mutant LIN-61 protein does not bind to H3K9me3 peptide (97). Therefore, it did not rescue loss of HPL-2 from the H3K9me3 peptide (figure 3.6). Thus, only WT LIN-61 protein could functionally replace endogenous LIN-61 in rescuing loss of HPL-2 binding to the H3K9me3 peptide. This shows that not only LIN-61, but also its binding to H3K9me3 peptide is important for the recovery of HPL-2 on H3K9me3 peptide. This is intriguing as native HPL-2, in spite of possessing a chromodomain, which is capable of binding $\mathrm{H} 3 \mathrm{~K} 9 \mathrm{me} 3$ in vitro, relies on LIN-61 for its recovery on $\mathrm{H} 3 \mathrm{~K} 9 \mathrm{me} 3$ peptide.

(A)

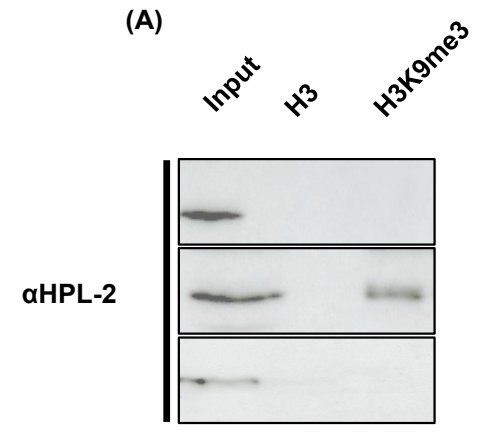

(B)

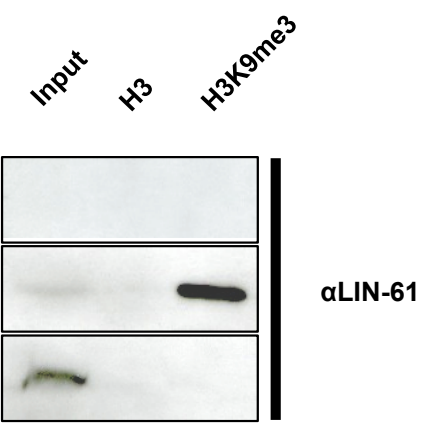

Figure 3.6: HPL-2 binding to H3K9me3 is mediated by LIN-61

Peptide pull-down assay using either H3 unmodified and H3K9me3 peptide was performed with lin-61 extract and either mock $T_{N} T$ mix (top lane), WT-LIN-61-T $T_{N}$ mix (middle lane) or MUT-LIN-61-TNT mix (bottom lane). Bound proteins were resuspended in sample buffer, resolved on SDS-PAGE and analyzed by western blotting using (A) anti-HPL-2 and (B) anti-LIN-61 antibodies.

Based on these results, we hypothesized that in $C$. elegans, the interaction between HPL-2 CD and H3K9me3 is negatively regulated by either 1) a post-translational modification, 2) allosteric regulation, 3) non-H3K9me3 methyllysine interaction or 4) a combination of these. HPL-2 phosphorylation has recently been reported (84). In order to test the first possibility, we made phosphomimic mutants of all known phosphorylation 
sites of HPL-2 (84). We prepared recombinant HPL-2T29E, HPL-2S55E, HPL-2T104E, HPL-2S155E and HPL-24E (all 4 sites mutated to glutamate) proteins and tested their interaction with $\mathrm{H} 3 \mathrm{~K} 9 \mathrm{me}$. We also included HPL-2Trimut (amino acids forming aromatic cage are replaced to alanine) as a negative control for the assay. We found that the phosphomimic mutations did not have any effect on CD-H3K9me3 interaction (figure 3.7A). In order to test the second possibility, we performed competitive depletion assay as described (figure 3.7B) with a goal to deplete the negative allosteric regulator. We did not find the recovery of HPL-2 on H3K9me3 even after 3 rounds of depletions of lin-61 extract (figure 3.7C).

(A)

(B) Binding of recombinant GST-HPL2

A)

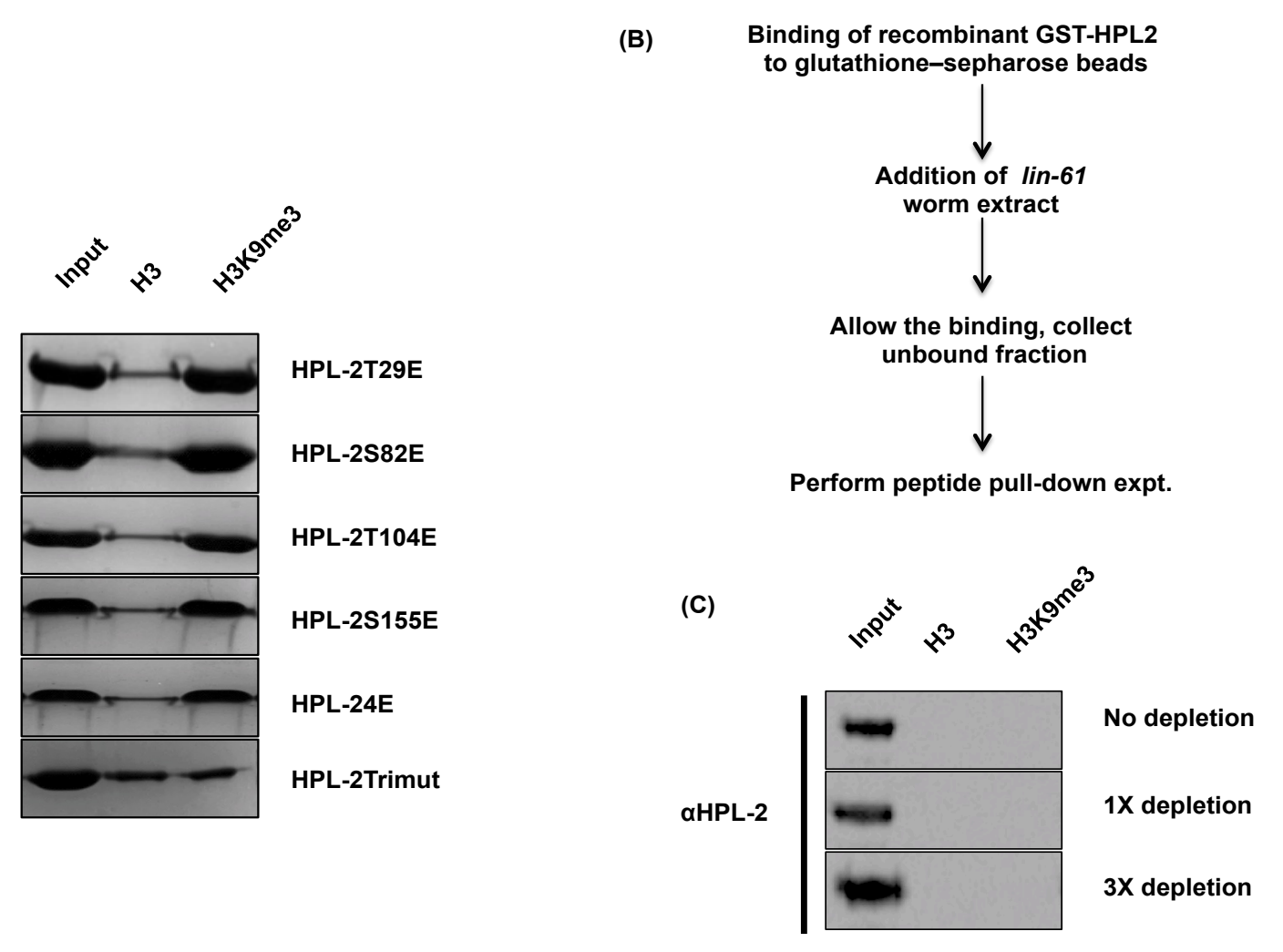

Figure 3.7: Negative regulation of HPL-2-H3K9me3 interaction

(A) Peptide pull-down assay was performed using $\mathrm{H} 3$ unmodified or $\mathrm{H} 3 \mathrm{~K} 9 \mathrm{me} 3$ peptide and recombinant phosphomimic mutants of HPL-2. HPL-2Trimut was used as negative control. (B) Schematic of competitive depletion assay. (C) Peptide pull-down assay was performed using unmodified $\mathrm{H} 3$ and H3K9me3 peptide and lin-61 mutant extract, undepleted (top panel), 1X depleted (middle panel) or $3 \mathrm{X}$ depleted (bottom panel). Bound proteins were detected by western blotting using rabbit anti-HPL-2 antibodies. 


\subsection{HPL-2 and LIN-61 associate with each other}

We found that HPL-2 depends on LIN-61 for its association with H3K9me3. We hypothesized that HPL-2 must associate with LIN-61 either directly or indirectly.

\subsubsection{HPL-2 and LIN-61 immunoprecipitate each other from C. elegans extract}

To test whether HPL-2 and LIN-61 interact with each other in vivo, we set up a coimmunoprecipitation assay from $C$. elegans nuclear extract. We found that both LIN-61 and HPL-2 co-immunoprecipitate each other from wild type worm extract (figure 3.8).

(A)

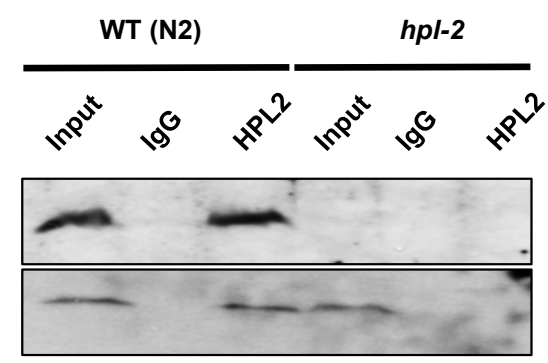

(B)

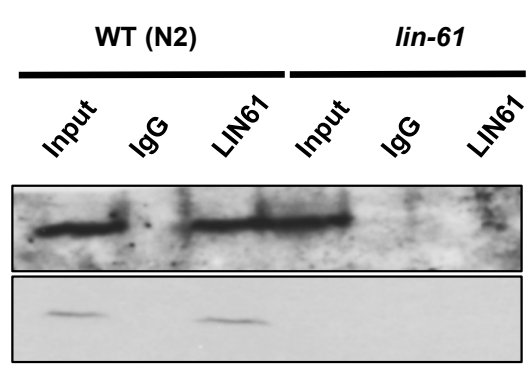

\section{Figure 3.8: HPL-2 and LIN-61 interact with each other in vivo}

Co-immunoprecipitaion assay was performed (A) with rabbit anti-HPL-2 antibodies in $\mathrm{N} 2$ and $h p l-2$ extracts or (B) with rabbit anti-LIN-61 antibodies in N2 and lin-61 extracts. Bound proteins were separated using SDS-PAGE and analyzed by western blotting using either anti-HPL-2 (top panel) or anti-LIN-61 (bottom panel)antibodies.

These results led us to conclude that LIN-61 and HPL-2 interact with each other in $C$. elegans extracts. It is therefore likely that this complex gets recruited on $\mathrm{H} 3 \mathrm{~K} 9 \mathrm{me} 3$ peptide in peptide pull-down assay via interaction of LIN-61 with H3K9me3.

\subsubsection{HPL-2 and LIN-61 directly interact with each other in vitro}

Although, we found that HPL-2 and LIN-61 form a protein complex, it is unclear whether they interact with each other directly or whether additional factor(s) mediate their interaction. To test this, we set up protein-protein interaction assays using the 
recombinant proteins. We expressed GST-tagged HPL-2 in E. coli and in-vitro translated LIN-61 protein in $T_{N} T$ system. We prepared either His-tagged LIN-61 or Myctagged LIN-61 for these assays. We tested HPL-2 and LIN-61 interaction using two different approaches. In the first approach, we immobilized either GST-HPL-2 or GSTHPL-1 on glutathione column and then passed $T_{N} T-L I N-61$ lysate over the beads. In another approach, we immobilized His-tagged LIN-61 to Ni-NTA beads and then passed recombinant HPL-2 protein.

We found that GST-HPL-2 recovers Myc-LIN61 and His-LIN-61 recovers GST-HPL2 specifically in these assays (figure 3.9). These results show that HPL-2 and LIN-61 directly interact with each other.

(A)

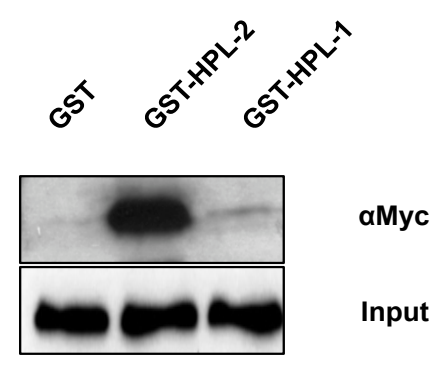

(B)

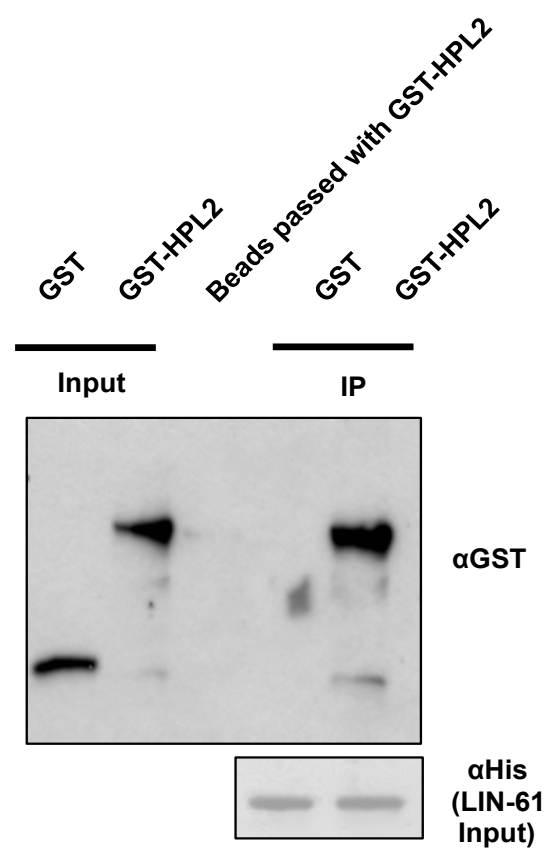

Figure 3.9: HPL-2 and LIN-61 directly interact with each other in vitro

(A) GST-pull-down assay was performed with recombinant GST-HPL-2 and $T_{N} T$ expressed Myc-tagged LIN-61. GST-HPL-2 protein was immobilized to the glutathione beads and allowed to bind to Myc-LIN-61. Bound proteins were detected by western blotting using anti-Myc antibodies. (B) Anti-His pull-down assay was performed with $\mathrm{T}_{\mathrm{N}} \mathrm{T}$ expressed His-tagged LIN-61 and GST-HPL-2 or only GST. His-LIN-61 was immobilized to Ni-NTA beads and allowed to bind GST-HPL-2. Bound proteins were detected by western blotting using anti-GST antibodies. 


\subsubsection{HPL-2 interacts with LIN-61 via its CSD}

Since, HPL-2 and LIN-61 directly interact with each other, we decided to map the regions in both proteins that are engaged in this interaction. We resorted to deletion mapping approach, where we constructed four deletion mutants of HPL-2 (figure 3.10A). We set up a GST-pull-down assay where we immobilized either GST-tagged full length HPL-2 or its deletion mutants (del 1, del 2, del 3 and del 4) and passed $\mathrm{T}_{\mathrm{N}} \mathrm{T}$ LIN-61 lysate over the glutathione column. We found that apart from full length HPL-2, del3 and del4 mutants also recover LIN-61 (figure 3.10B). As seen in figure 3.10A, del3 and del4 both posses intact CSD, which suggests that CSD is involved in the interaction of HPL-2 with LIN-61.

(A)

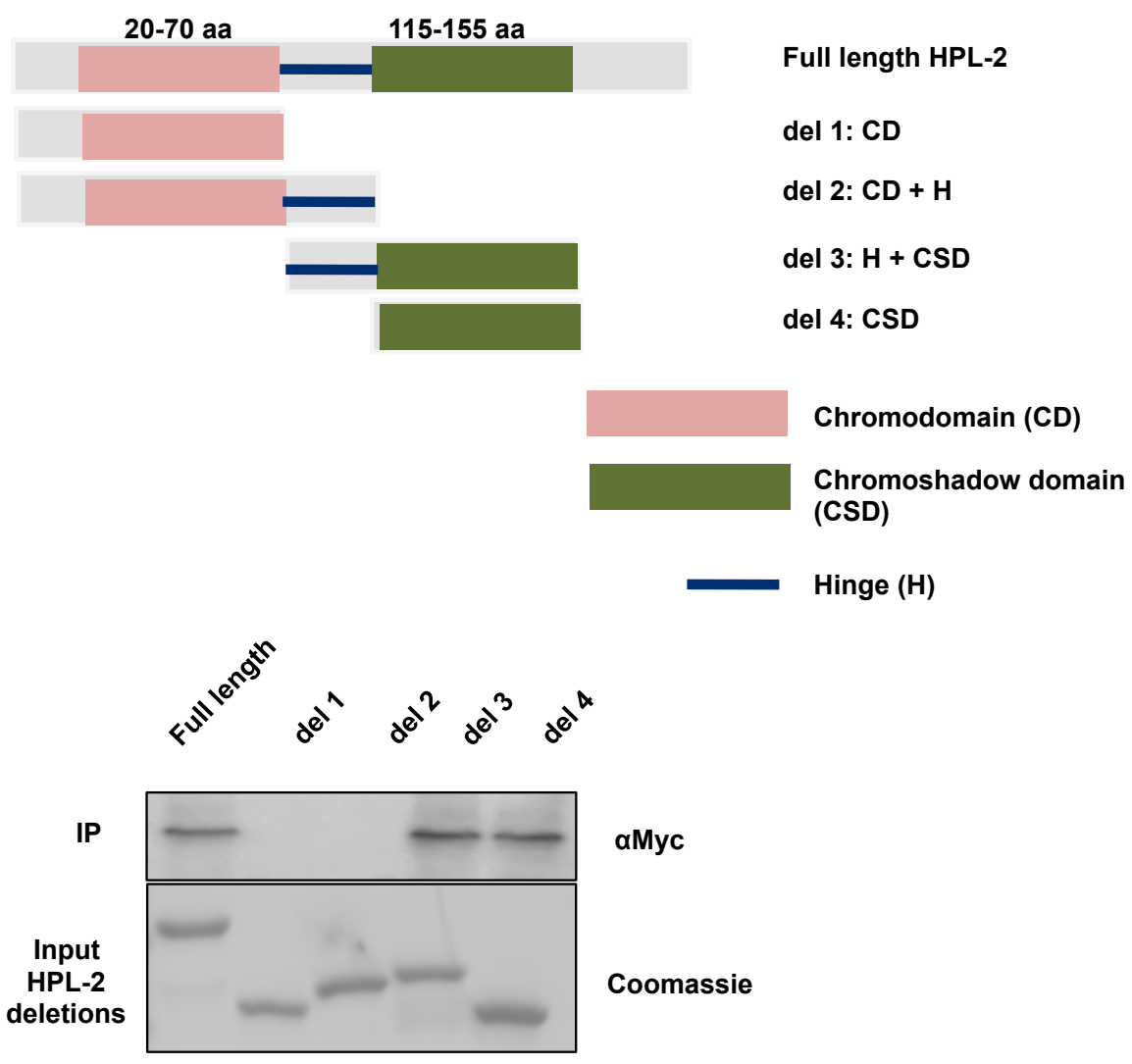

Figure 3.10: HPL-2 interacts with LIN-61 via its chromoshadow domain (CSD) in vitro

(A) Scheme showing domain organization of full length HPL-2 and various deletions mutants of HPL-2. (B) GST-pull-down assay was performed with recombinant GSTHPL-2 or deletion mutants (del1 to del4) and $T_{N}$ T expressed Myc-tagged LIN-61. GST- 
HPL-2 protein/mutants were immobilized to the glutathione beads and allowed to bind to Myc-LIN-61. Bound proteins were detected using western blotting using anti-Myc antibodies.

Since, the structural data for LIN-61 suggests that four MBT domains of the protein inter-digitate to form a tertiary structure that is functional, deletion-mapping approach was not feasible for LIN-61. We therefore, decided to screen the known point mutants of LIN-61 that potentially lack the ability to interact with HPL-2 without disrupting the structure of LIN-61.

We tested five different point mutants of LIN-61, which have been reported earlier (99). However, we found that none of these point mutations had any effect on the LIN61-HPL-2 interaction (figure 3.11).

\subsection{H3K9me3-LIN-61-HPL-2 signaling regulates vulva development via SynMuv B pathway}

Next, we decided to elucidate the biological significance of HPL-2-LIN-61-H3K9me3 interaction. It has been reported that both $h p l-2$ and lin-61 are required for normal vulva and germline development $(97,99)$. We asked whether any defects in vulva development are the result of loss of interaction(s) in HPL-2-LIN-61-H3K9me3 axis.

\subsubsection{Characterization of various lin-61 alleles}

We started characterizing all available lin-61 mutant alleles described (99). All these alleles were identified in a genetic screen for SynMuv B pathway genes. Five of these alleles are point mutants in the LIN-61 protein. All these alleles encoded for proteins that had reduced stability in the corresponding strains except one allele, lin-61 (n3447). This allele encodes for LIN-61S354N mutant protein where a serine in the third MBT domain is exchanged to asparagine. When translated in vitro using $\mathrm{T}_{\mathrm{N}} \mathrm{T}$ system in rabbit reticulocyte and tested for it's binding to H3K9me3 with a peptide pull-down assay, we found that its interaction with $\mathrm{H} 3 \mathrm{~K} 9 \mathrm{me} 3$ is severely reduced. With the peptide pull-down assays for all known LIN-61 point mutants reported earlier (97), we qualitatively evaluated the binding of these LIN-61 point mutants to H3K9me3 (table 3.1). 
Table 3.1: Various LIN-61 mutants and the strength of their interaction with H3K9me3

\begin{tabular}{|c|c|}
\hline LIN-61 mutants & Binding to H3K9me3 \\
\hline WT & +++ \\
\hline D428A & ++ \\
\hline F452A & - \\
\hline W455A & + \\
\hline F459A & - \\
\hline F452AW455AF459A & +++ \\
\hline P132S (n3624) & + \\
\hline F247S (n3736) & - \\
\hline G250E (n3807) & + \\
\hline S354N (n3447) & - \\
\hline G445R (n3922) & \\
\hline
\end{tabular}

In the lin-61 (n3447) mutant strain, the protein is expressed at similar levels to WT LIN61 protein in N2 strain (99). This strain provided us with a unique tool to test the binding of HPL-2 to H3K9me3 in lin-61 (n3447) extract. As expected, the LIN-61S354N protein had severely reduced binding to $\mathrm{H} 3 \mathrm{~K} 9 \mathrm{me} 3$ peptide in the extract with concomitant loss of HPL-2 binding to H3K9me3 (figure 3.11A). Nonetheless, LIN-61 S354N when tested for its interaction with HPL-2, showed similar interaction compared to WT LIN-61 (figure 3.11B). 
(A)

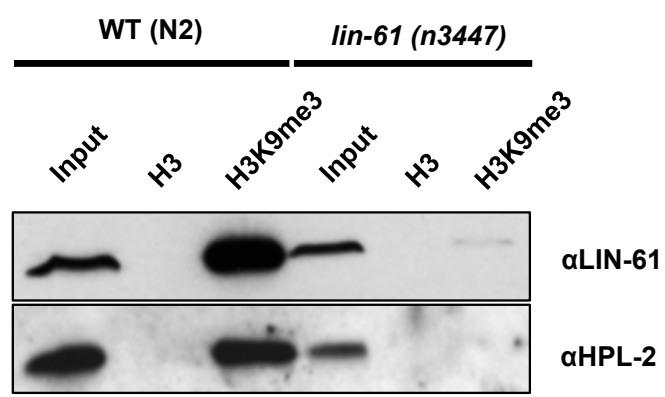

(B)

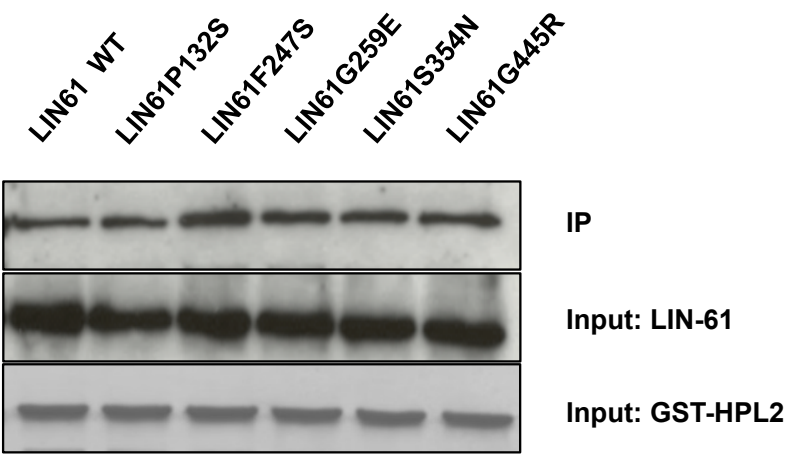

Figure 3.11: LIN-61 and its interaction with $\mathrm{H} 3 \mathrm{~K} 9 \mathrm{me} 3$ is required for recovery of HPL-2 on H3K9me3 peptide

(A) Peptide pull-down assay was performed with H3 unmodified or H3K9me3 peptide with either N2 or lin-61 (n3447) extract. Bound proteins were detected by western blotting using anti-LIN-61 antibodies (top panel) or anti-HPL-2 antibodies (bottom panel). (B) GST pull-down assay with GST-HPL-2 and various LIN-61 mutant proteins. Bound proteins were detected by western blotting using anti-Myc antibodies (top panel). Middle panel: Input for LIN-61 (10\%). Bottom panel: Input for GST-HPL-2.

We could not identify any loss of function mutant for HPL-2-LIN-61 interaction. We conclude that in lin-61 (n3447), LIN-61-H3K9me3 interaction is abolished. Consequently, HPL-2 binding to H3K9me3 peptide is alsolost. 


\subsubsection{H3K9me3-dependent LIN-61-HPL-2 pathway belongs to SynMuv B class of vulva development}

Since, we identified a lin-61 (n3447) mutant allele that is a loss of function mutant for H3K9me3-LIN-61-HPL-2 biochemical interaction, we asked whether this mutation has any effect on the vulva development pathways in C. elegans. We combined the lin-61 (n3447) allele with SynMuv A (lin-15a and lin-8) and SynMuv B (met-2) mutants. We found that in SynMuv A mutant background, the allele lin-61 (n3447) shows strong genetic interaction (table 3.2) suggesting that H3K9me3-LIN-61-HPL-2 biochemical pathway belongs to SynMuv B class.

Next, we hypothesized that $h p l-2$ exerts its effect on vulva development via the H3K9me3-LIN-61-HPL-2 pathway. If this is the sole pathway that exists in C. elegans through which hpl-2 regulates vulva development, the allele lin-61 (n3447) should be epistatic to hpl-2 (tm1489). We found that, lin-61 (n3447) genetically interacts with hpl-2 (tm1489) (table 3.2). However, the extent of genetic interaction was less compared to protein null alleles of HPL-2 and LIN-61 (hpl-2 (tm1489); lin-61 (tm2649)).

We confirmed these findings using another lin-61 (n3809) allele, a protein null mutant that also showed greater penetrance as compared to the lin-61 (n3447) allele. From these results, we concluded that both HPL-2 and LIN-61 regulate vulva development through H3K9me3-LIN-61-HPL-2 pathway.

To test, whether HPL-2 and LIN-61 also regulate vulva development independent of H3K9me3 pathway, we chose met-2 (tm4256) allele because met-2 encodes for a H3K9 methyltransferase and is a C. elegans homologue of SUV39H enzymes. The allele lin61 (tm2649) is shown to genetically interact with met-2 (tm4256) (97). We found that all three alleles of lin-61 show slight genetic interaction with met-2 at $25^{\circ} \mathrm{C}$ (table 3.2 ). From these results, we concluded that both HPL-2 and LIN-61 also regulate vulva development independent of H3K9me3. 
Table 3.2: SynMuv analyses of various lin-61 mutants

\begin{tabular}{|c|c|c|}
\hline \multirow[t]{2}{*}{ Genotype } & \multicolumn{2}{|c|}{ SyuMuv \pm SD } \\
\hline & $20^{\circ} \mathrm{C}$ & $25^{\circ} \mathrm{C}$ \\
\hline $\operatorname{lin}-61$ (n3447) & 0.0 & $0.4 \pm 0.51$ \\
\hline lin-61 (n3809) & $0.2 \pm 0.42$ & $0.3 \pm 0.48$ \\
\hline $\operatorname{lin}-61$ (tm2649) & $0.1 \pm 0.31$ & $0.1 \pm 0.31$ \\
\hline lin-61 (tm2649); ;in-15a (n767) & $80.39 \pm 1.8$ & $98.02 \pm 0.5$ \\
\hline $\operatorname{lin}-61$ (tm2649) ;lin-8 (n2731) & 0.0 & $0.5 \pm 0.7$ \\
\hline lin-61 (tm2649) ; hpl-2 (tm1489) & $0.85 \pm 0.04$ & $79.1 \pm 3.01$ \\
\hline lin-61 (tm2649); ;met-2 (tm4256) & $0.64 \pm 0.11$ & $16.73 \pm 1.55$ \\
\hline lin-61 (n3447); lin-15a (n767) & $23.7 \pm 1.94$ & $99.3 \pm 1.05$ \\
\hline lin-61 (n3447); lin-8 (n2731) & $7.1 \pm 1.66$ & $81.1 \pm 3.34$ \\
\hline lin-61 (n3447); hpl-2 (tm1489) & $0.49 \pm 0.21$ & $52.39 \pm 0.81$ \\
\hline lin-61 (n3447); met-2 (tm4256) & $0.51 \pm 0.04$ & $8.27 \pm 0.62$ \\
\hline lin-61 (n3809); lin-15a (n767) & $95 \pm 2$ & $99.7 \pm 0.67$ \\
\hline lin-61 (n3809); lin-8 (n2731) & $94.4 \pm 2.17$ & $97.2 \pm 1.31$ \\
\hline lin-61 (n3809); hpl-2 (tm1489) & $0.5 \pm 0.2$ & $79.23 \pm 2.45$ \\
\hline lin-61 (n3809); met-2 (tm4256) & $0.33 \pm 0.07$ & $11.87 \pm 0.39$ \\
\hline
\end{tabular}

All animals scored were raised at $20^{\circ} \mathrm{C}$ or $25^{\circ} \mathrm{C}$ as indicated. Three trials of independent worm growth per genotype were conducted. The muv phenotype was analyzed using a dissecting microscope and animals were scored as muv if one or more ectopic ventral protrusions (pseudovulvae) were observed. SD: Standard deviation, $n$ : total number of worms analyzed. 


\subsection{LIN-13 interacts with HPL-2 and LIN-61}

We next wanted to identify the possible mechanisms by which $h p /-2$ could regulate vulva development apart from its role in LIN-61-H3K9me3 pathway. It has been reported earlier that HPL-2 and LIN-13 directly interact with each other and regulate vulva development (117). Moreover, during the course of this work, another study reported that HPL-2, LIN-13 and LIN-61 form a unique 'SynMuv B heterochromatin complex' that is involved in the repression of germline RNAi pathways in somatic cells (105). We asked whether HPL-2 interacts with LIN-13 independent of its interaction with LIN-61. We obtained the GFP-tagged strains for HPL-2, LIN-61 and LIN-13 and FLAG-tagged strain for LIN-61 (105) and validated the GFP tagged protein function by rescue experiments in double mutants for SynMuv phenotype (data not shown).

\subsubsection{HPL-2/LIN-61/LIN-13 co-immunoprecipitate each other}

We performed immunoprecipitation assay using all three GFP tagged strains. We found that all three factors, HPL-2, LIN-61 and LIN-13 were able to immunoprecipitate each other (figure 3.12). 
(A)

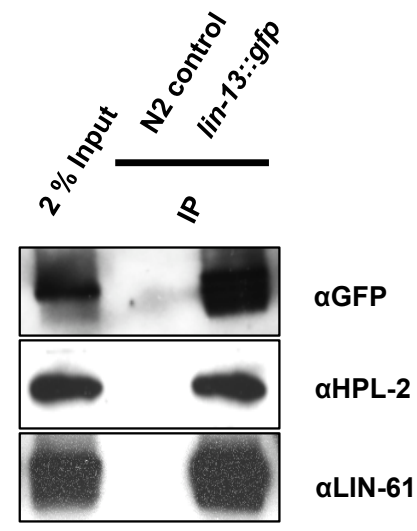

(B)

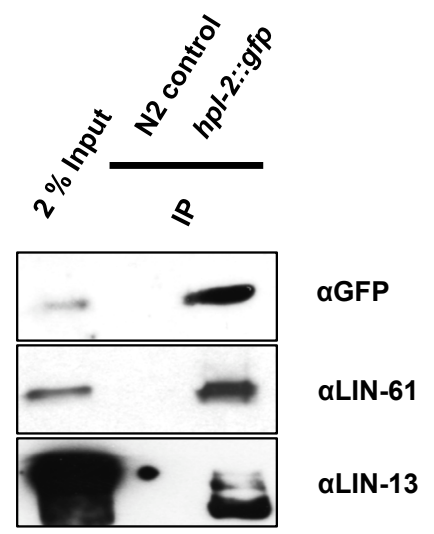

(C)

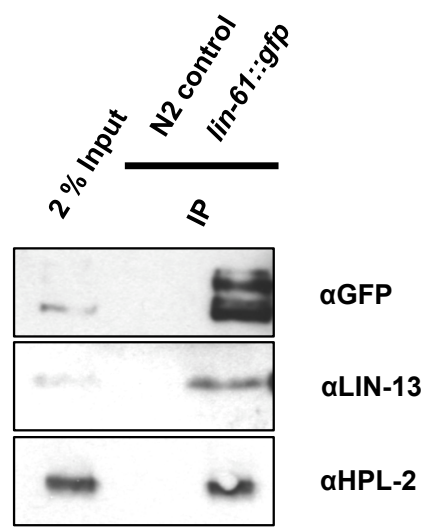

Figure 3.12: HPL-2/LIN-61/LIN-13 co-immunoprecipitate each other

Anti-GFP immunoprecipitation was performed using GFP_Trap with either N2 extract (negative control in all cases) or (A) lin-13::gfp extract, (B) hpl-2::gfp extract, (C) lin$61:: g f p$ extract. Bound proteins were resolved by SDS-PAGE and detected by western blotting using anti-GFP, anti-HPL-2, anti-LIN-61 or anti-LIN-13 antibodies.

\subsubsection{Interaction between HPL-2 and LIN-13 is reduced in the absence of LIN-61} while interaction between LIN-13 and LIN-61 is lost in the absence of HPL-2

While it was clear that HPL-2, LIN-61 and LIN-13 interact with each other, we did not know whether these form a single complex. We thus, asked whether all three factors directly interact with each other or there exists a hierarchy in formation of a possible protein complex. 
We crossed hpl-2::gfp strain in lin-61 (tm2649) mutant background, lin-61::gfp strain in hpl-2 (tm1489) mutant background and lin-13::gfp strain in lin-61 (tm2649) and hpl-2 (tm1489) mutant backgrounds. Using immunoprecipitation assays, we tested the effect of loss of HPL-2 and LIN-61 on interaction among three factors. lin-13 deletion alleles are not viable (96) and hence, we resorted to lin-13 knock-down in order to test the effect of LIN-13 on interaction among three factors.

We tested the interaction between LIN-13 and HPL-2 in the absence of LIN-61. Likewise, we also tested the interaction between LIN-13 and LIN-61 in the absence of HPL-2. We found that the interaction between LIN-61 and LIN-13 is lost in the absence of HPL-2. This loss of interaction was observed in both cases, where we either immunoprecipitated LIN-13 (figure 3.13A) or LIN-61 (figure 3.13B), both in hpl-2 (tm1489) background.

We performed similar experiment to test the interaction between LIN-13 and HPL-2 in the absence of LIN-61. We observed that the interaction between these two proteins was significantly reduced in the absence of LIN-61 (figure 3.13C and 3.13D) under the conditions we used.

We conclude that LIN-61 and LIN-13 do not interact with each other directly, and rather depend on HPL-2 for their interaction. This indicates that HPL-2, LIN-61 and LIN-13 form a complex where HPL-2 bridges LIN-61 and LIN-13. 
(A)
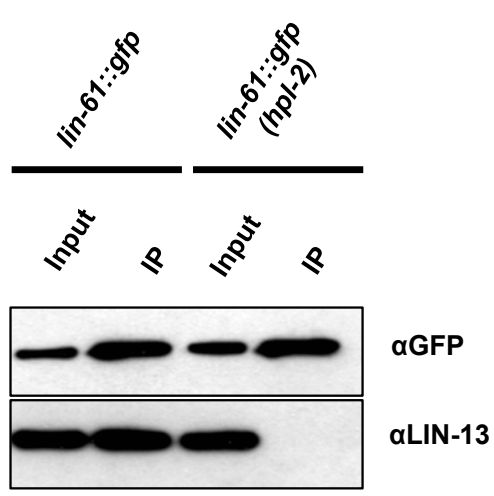

(C)

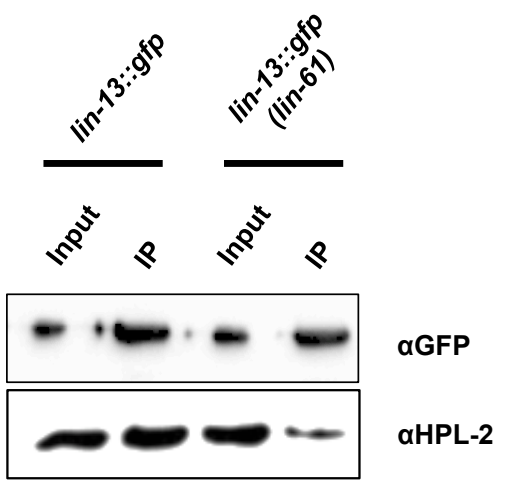

(B)
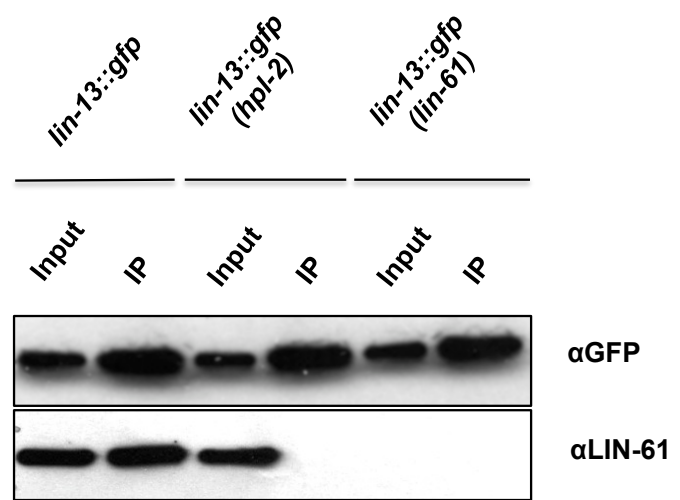

(D)

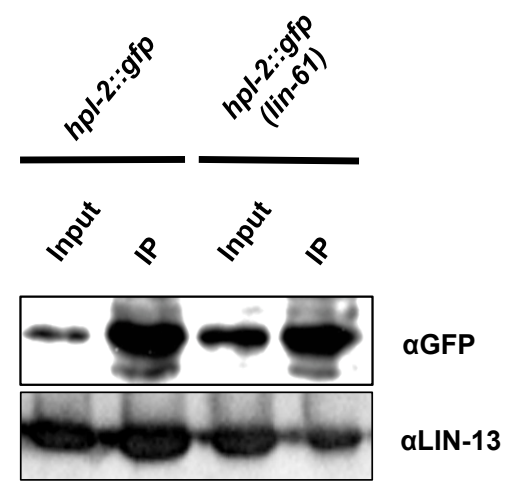

Figure 3.13: Interaction between LIN-13 and LIN-61 is lost in the absence of HPL-2 while the interaction between LIN-13 and HPL-2 is reduced in the absence of LIN61

Anti-GFP immunoprecipitation was performed using GFP_Trap with (A) lin-61::gfp extract or lin-61::gfp;hpl-2(tm1489) extract, (B) lin-13::gfp extract or lin-13::gfp; hpl-2 (tm1489) extract or lin-13::gfp; lin-61 (tm2649) extract, (C) lin-13::gfp extract or lin13::gfp; lin-61 (tm2649) extract, (D) hpl-2::gfp extract or hpl-2::gfp; lin-61 (tm2649) extract. Bound proteins were resolved by SDS-PAGE and detected by western blotting by anti-GFP, anti-HPL-2, anti-LIN-61 or anti-LIN-13 antibodies. 


\subsubsection{LIN-13 is H3K9me3 associated protein}

We showed that LIN-13, HPL-2 and LIN-61 form a complex. Our earlier results demonstrated that HPL-2 and LIN-61 bind to H3K9me3 peptide. We therefore, tested the recovery of LIN-13 on H3K9me3 peptide in a peptide pull-down assay. We found that LIN-13 is recovered specifically on H3K9me3 peptide in wild type, N2 extract (3.14).

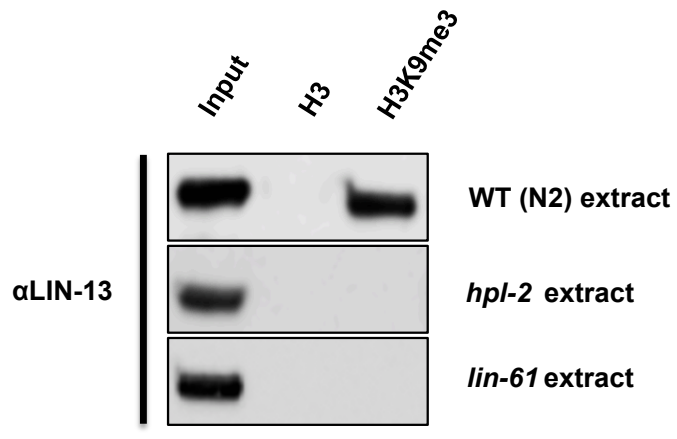

Figure 3.14: LIN-13 is H3K9me3 associated protein

Peptide pull-down assay was performed with either H3 unmodified or H3K9me3 peptide using N2 extract (top panel), hpl-2 (tm1489) extract (middle panel) or lin-61 (tm2649) extract (bottom panel). Bound proteins were resolved by SDS-PAGE and detected by western blotting using anti-LIN-13 antibodies.

\subsubsection{LIN-13 recovery on H3K9me3 is dependent on both LIN-61 and HPL-2}

We asked whether the recovery of LIN-13 on H3K9me3 peptide is dependent upon LIN61 or HPL-2 or both. We performed peptide pull-down assays using N2, lin-61 and hpl-2 extracts. Interestingly, we did not detect LIN-13 on H3K9me3 peptide in both lin-61 and hpl-2 mutant extracts (figure 3.14). We conclude that LIN-13 recovery on H3K9me3 peptide depends on both LIN-61 and HPL-2.

We then performed complementation assay, where we added recombinant HPL-2 and $\mathrm{T}_{\mathrm{N}} \mathrm{T}$-expressed LIN-61 in mutant $h p /-2$ and lin-61 extracts respectively to test whether these can complement endogenous HPL-2 and LIN-61 for the recovery of LIN-13 on H3K9me3 peptide.

We added $T_{N}$ T-expressed WT LIN-61 or MUT LIN-61 to lin-61 mutant extracts. We found that only when WT LIN-61 was added, LIN-13 was able to recover on H3K9me3 (figure 3.15A, B). We also added recombinant GST-HPL-2 in hpl-2 mutant extracts and found that LIN-13 was able to recover on H3K9me3 peptide (figure 3.15C). 

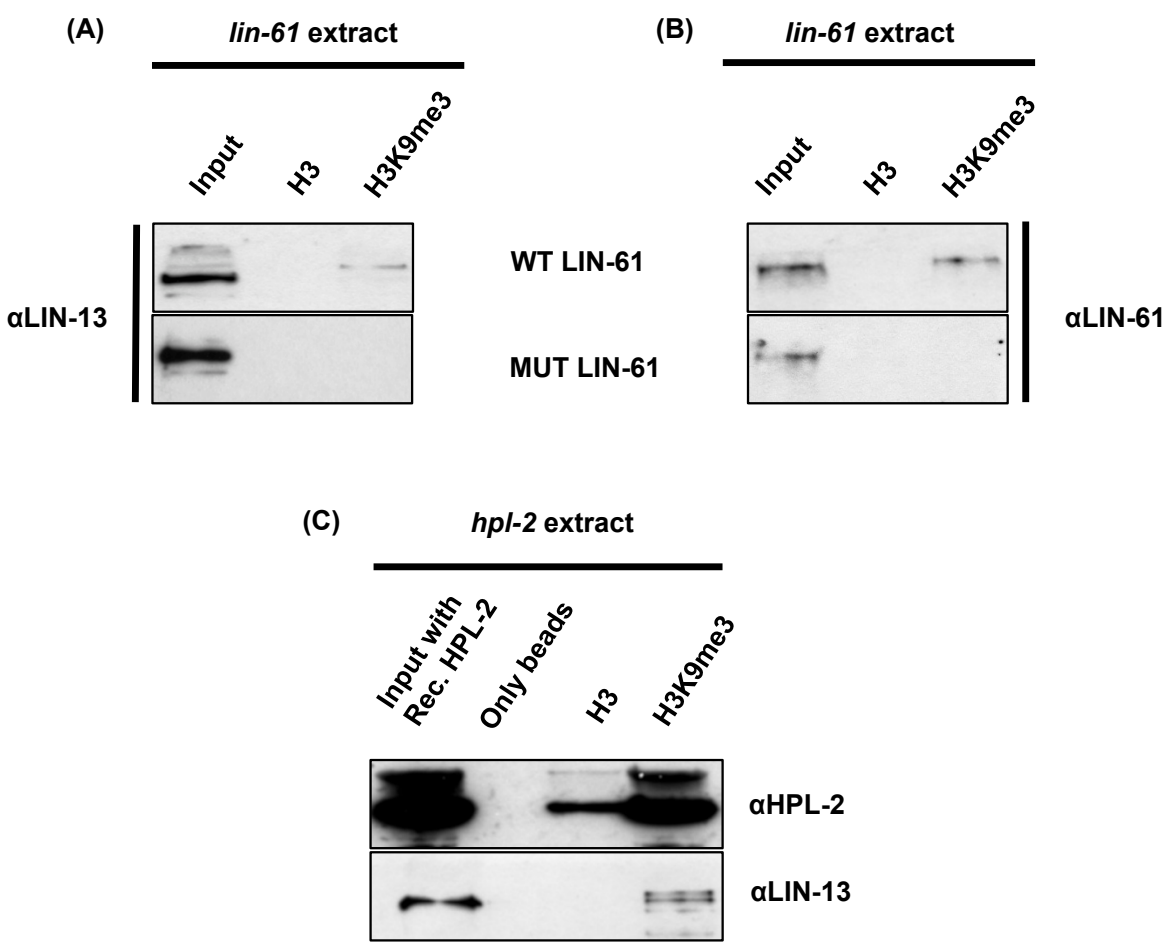

\section{Figure 3.15: LIN-13 recovery to H3K9me3 is dependent on both HPL-2 and LIN-61}

Peptide pull-down assays were performed with either $\mathrm{H} 3$ unmodified or H3K9me3 peptide using (A, B) lin-61 (tm2649) extract or (C) hpl-2 (tm1489) extract. $\mathrm{T}_{\mathrm{N}} \mathrm{T}$ expressed WT LIN-61 (top panel) or MUT LIN-61 (bottom panel) was added to the lin61 (tm2649) extract. Bound proteins were detected using (A) anti-LIN-13 or (B) antiLIN-61 antibodies. (C) Recombinant HPL-2 was added to hpl-2 (tm1489) extract. Bound proteins were detected by western blotting using anti-HPL-2 (top panel) and anti-LIN-13 (bottom panel) antibodies.

We conclude that LIN-13 associates with H3K9me3 downstream of HPL-2 and LIN-61. Earlier, we have found that HPL-2 depends on LIN-61 for its association with H3K9me3. Taken together, these results suggest that likely H3K9me3 peptides directly bind LIN61. LIN-61 binds to HPL-2, which in turn binds LIN-13. 


\subsection{HPL-2/LIN-61/LIN-13 complex}

We propose that HPL-2, LIN-61 and LIN-13 form a complex where HPL-2 interacts with LIN-61 via its CSD. Interestingly, it is reported that LIN-13 also interacts with HPL-2 CSD (117). It is not known whether HPL-2 interacts with LIN-61 and LIN-13 with the same CSD molecule or its multimerization is required for simultaneous interaction with LIN-13 and LIN-61.

\subsubsection{Full length HPL-2 is required for HPL-2/LIN-61/LIN-13 complex formation}

If HPL-2 engages with both LIN-61 and LIN-13 via single CSD molecule simultaneously, then it is presumed that $C D$ is not required for the LIN-61/HPL-2/LIN-13 complex formation. If this is true, a simple trimolecular complex can be proposed. To test this, we added recombinant CSD alone to the wild type worm extracts in a competition peptide pull-down assay. If CSD alone is sufficient for the HPL-2/LIN-61/LIN-13 complex formation, CSD should effectively compete out endogenous HPL-2 without affecting the interaction of LIN-61 and LIN-13 with H3K9me3 peptide.

We found that, in a dose dependent manner, CSD effectively competes out endogenous HPL-2 from the pull-down reaction (figure 3.16A). Addition of CSD did not affect the recovery of LIN-61 on H3K9me3 peptide, which is in consistence with our earlier results that LIN-61 binds H3K9me3 peptide independent of HPL-2 (figure 3.16A). Interestingly, addition of CSD resulted in loss of LIN-13 from H3K9me3 peptide (figure 3.16A). This suggests that CSD alone is insufficient to recruit LIN-13 to H3K9me3 peptide. We conclude that full length HPL-2 is required for LIN-61/HPL-2/LIN-13 complex formation where CSD interacts with both LIN-61 and LIN-13.

As a control experiment, we added recombinant GST-HPL-2-CSD in a peptide pulldown assay containing recombinant full length GST-HPL-2 and H3K9me3 peptide and found that CSD does not compete with full length HPL-2 and does not kick HPL-2 out from the pull-down reaction by physically sequestering it (figure 3.16B). It rather competes with endogenous HPL-2 in the extract for a binding site on LIN-61. 
(A) H3K9me3

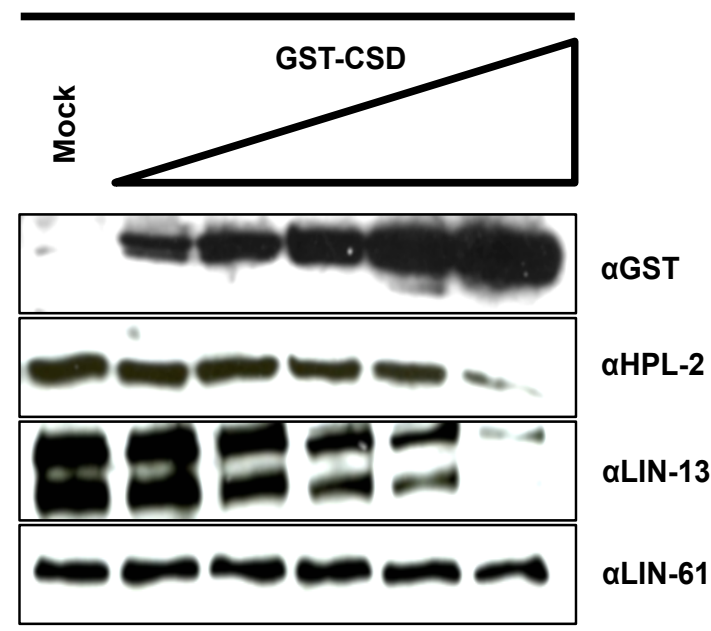

(B)
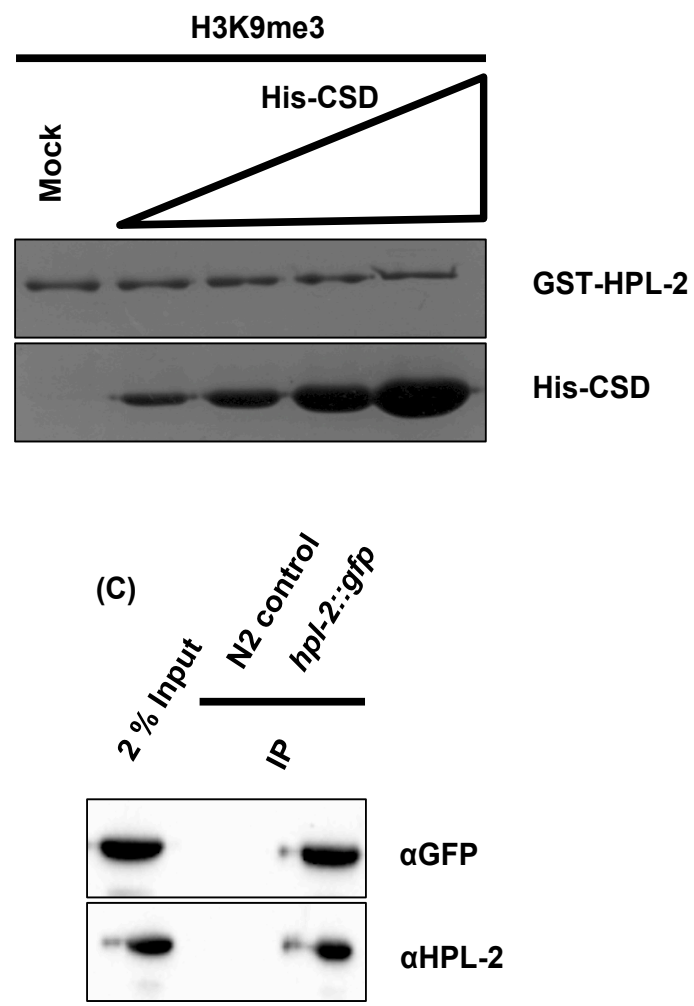

Figure 3.16: Recombinant CSD competes with endogenous HPL-2 for H3K9me3 binding and HPL-2 forms multimers in vivo.

(A) Peptide pull-down assay was performed with H3K9me3 peptide using N2 extract. Recombinant GST-CSD was added in a competition assay in increasing amounts $0 \mu \mathrm{g}$, $0.5 \mu \mathrm{g}, 2 \mu \mathrm{g}, 5 \mu \mathrm{g}, 25 \mu \mathrm{g}$ and $75 \mu \mathrm{g}$ to extract. Bound proteins were then resolved on SDS-PAGE and detected by western blotting using anti-GST (top panel), anti-HPL-2 ( $2^{\text {nd }}$ panel), anti-LIN-13 ( $3^{\text {rd }}$ panel) and anti-LIN-61 (bottom panel) antibodies. (B) Peptide pull-down assay was performed with H3K9me3 peptide using recombinant GST-HPL-2. His-CSD was added in a competition assay in increasing amounts $0 \mu \mathrm{g}$, $0.5 \mu \mathrm{g}, 5 \mu \mathrm{g}, 25 \mu \mathrm{g}$ and $75 \mu \mathrm{g}$. Bound proteins were then resolved on SDS-PAGE and detected using coomassie blue staining. (C) GFP immunoprecipitation assay from $\mathrm{hpl}$ 2::gfp nuclear extract. Bound proteins were resolved on SDS and detected by western blotting with anti-GFP (top panel) or anti-HPL-2 (bottom panel) antibodies. 


\subsubsection{HPL-2 forms multimers in vivo}

We hypothesized that HPL-2 forms multimers and via multiple CSD molecules, HPL-2 tethers LIN-61 and LIN-13 together in a single complex. In order to test this, we performed immunoprecipitation assays with GFP-tagged HPL-2 using extracts from transgenic worm strains having GFP-HPL-2 protein. We found that HPL-2-GFP is able to immunoprecipitate endogenous HPL-2 (figure 3.16C). We conclude that HPL-2 forms multimers in vivo and likely its multimerization is required for HPL-2/LIN-61/LIN-13 complex formation.

\section{6 lin-13 RNAi}

Next, we proposed that if LIN-13 interacts with only CSD of HPL-2, independent of HPL2-CSD-LIN-61 interaction, loss of LIN-13 should not affect the interaction between HPL2 and LIN-61. To test this, we resorted to lin-13 RNAi since lin-13 null mutants are not viable (96).

We performed lin-13 RNAi using feeding assay. In N2 worms, a single generation of RNAi experiment did not result in any observable phenotype and also resulted in depletion lin-13 mRNA only to $57 \pm 30 \%$ of control levels with a lot of variability (data not shown). This could be due to the fact that maternal LIN-13 is sufficient for its function (96). Moreover, protein sequence analysis also predicted a high protein half-life for LIN13 (data not shown). We thus, performed a two-generational RNAi, where we fed worms with RNAi bacteria for two consecutive generations. 


\subsection{1 lin-13 RNAi in wild type N2 worms causes highly penetrant muv phenotype and sterility}

Upon knock-down of lin-13 for two consecutive generations in N2 strain, F1 worms showed sterility and highly penetrant muv phenotype, which is consistent with earlier studies (96). We observed highly penetrant muv phenotype, 96.3 $\pm 0.3 \%(P<0.0001$, student's t-test) with $100 \%$ sterility in animals fed with lin-13-pL4440 as compared to control animals fed with pL4440 alone (figure 3.17).

(A)

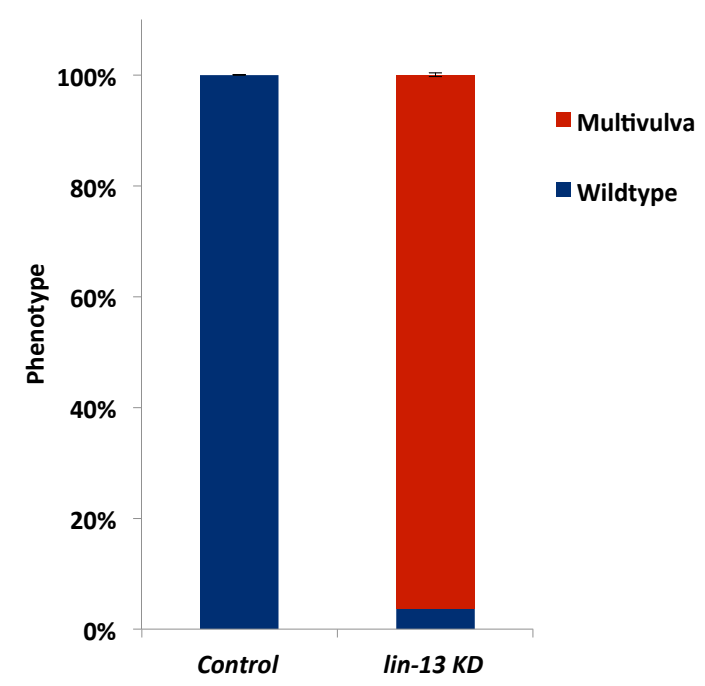

(B)

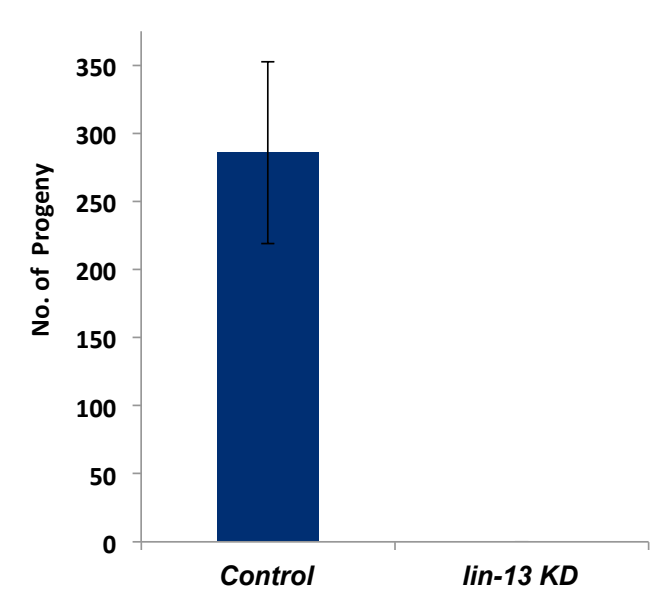

Figure 3.17: lin-13 RNAi results in muv phenotype and sterility in C. elegans

N2 worms were fed with either pL4440 or lin-13-pL4440 bacteria for two generations. F2 worms were scored. (A) Either WT or muv phenotype (worms with more than one vulval projections). Percent muv phenotype in N2 worms was scored in 3 independent assays (students' t-test, $\mathrm{P}<0.0001$ ). (B) Average no. of progeny from 29 and 50 worms fed with either pL4440 and lin-13-pL4440 bacteria respectively (student's t-test, $\mathrm{P}<0.0001)$. Error bars represent \pm standard deviation. lin-13 RNAi experiments were performed with Elizabeth Miller. 


\subsection{2 lin-13 RNAi in wild type N2 worms leads to decrease in HPL-2 and LIN-61} protein levels but does not affect their mRNA levels

We confirmed the knock-down of lin-13 by measuring the levels of lin-13 mRNA as well as protein (figure 3.18A, B). In lin-13 RNAi worms, we observed a significant decrease in mRNA levels of lin-13 down to $25 \pm 4.4 \%$ of the control worms and also a corresponding decrease in protein levels of LIN-13. We also observed a slight increase in the mRNA levels of $h p l-2(119 \pm 22 \%)$ and lin-61 (124 $\pm 15 \%)$ compared to control worms (3.18A). Surprisingly, loss of LIN-13 led to a pronounced decrease in protein levels of HPL-2 and a slight decrease in protein levels of LIN-61 (figure 3.18B). This limited us to perform the immunoprecipitation assay upon lin-13 RNAi to test the interaction between HPL-2 and LIN-61 in the absence of LIN-13.

(A)

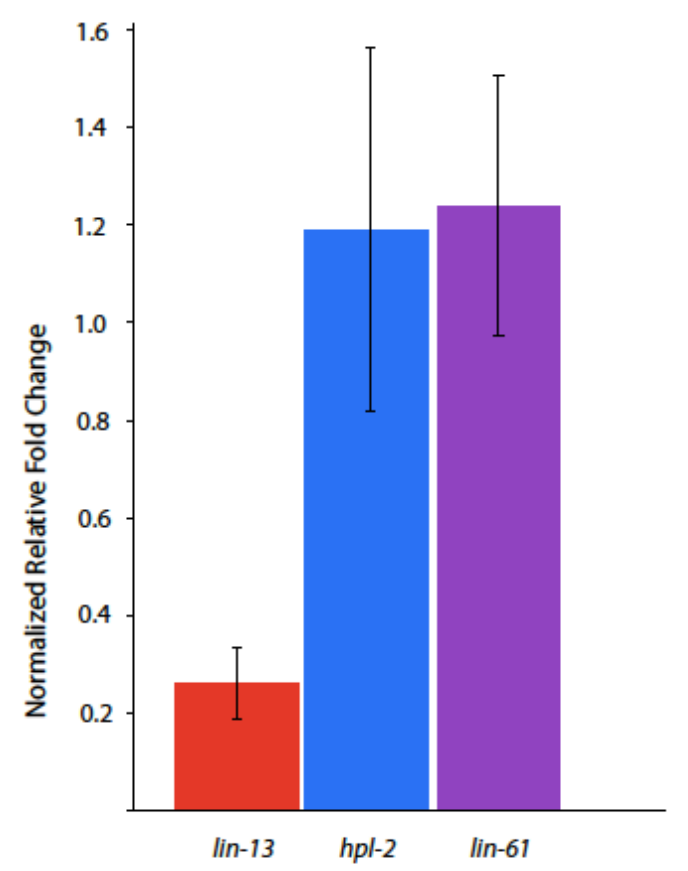

(B)
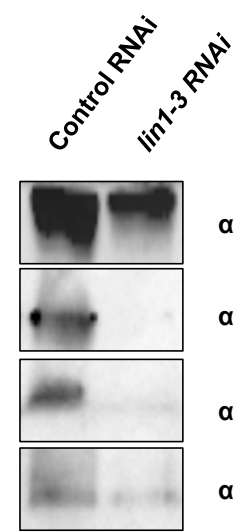

$\alpha \mathrm{H} 3$

$\alpha \operatorname{LIN}-13$

aHPL-2

$\alpha$ LIN-61
(C)

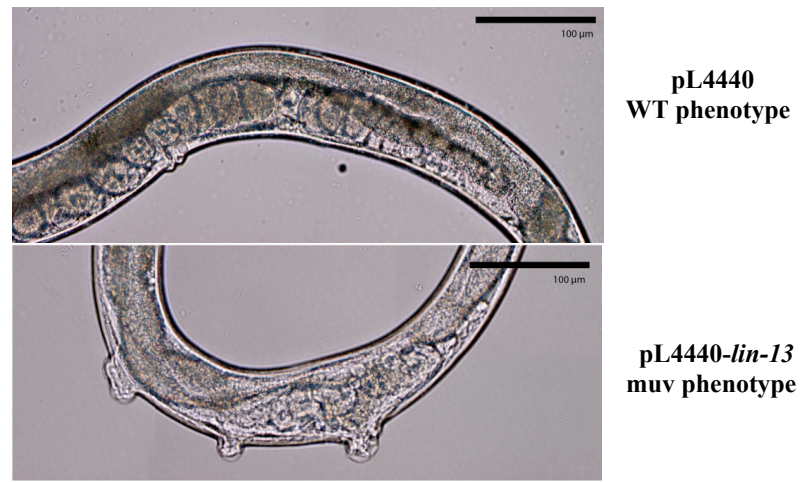

Figure 3.18: lin-13 RNAi leads to decrease in HPL-2 and LIN-61 protein levels, but not mRNA levels 
(A) Real time RT-PCR analysis of RNA species isolated from N2 worms fed with either pL4440 or lin-13-pL4440 bacteria. Fold change values wee obtained after normalization with housekeeping gene, rpl-26. Primers for lin-13, hpl-2 or lin-61 were used. (B) Western blot analysis of LIN-13, HPL-2 and LIN-61 protein levels of N2 worms fed with either pL4440 or lin-13-pL4440 bacteria. (C) pL4440 RNAi worms (left) with WT phenotype and pL4440-lin-13 RNAi worms with muv phenotype. lin-13 RNAi experiments were performed with Elizabeth Miller.

Since, levels of LIN-61 were just slightly affected upon lin-13 RNAi, we performed peptide pull-down assay with worm extracts from lin-13 RNAi worms and control RNAi worms to test the effect of loss of LIN-13 on LIN-61-H3K9me3 interaction. Our earlier results suggest that LIN-61-H3K9me3 interaction is independent of HPL-2 as such. Therefore, loss of LIN-13 should have no effect on LIN-61-H3K9me3 interaction. Expectedly, LIN-61 binding to H3K9me3 remained unaffected by loss of LIN-13 (figure 3.19). However, HPL-2 binding to H3K9me3 was diminished (figure 3.19). We conclude that the loss of HPL-2 association to H3K9me3 peptide is due to a decrease in HPL-2 protein levels upon lin-13 knock-down. However, the possibility of LIN-13 enhancing HPL-2-LIN-61 interaction could not be ruled out.

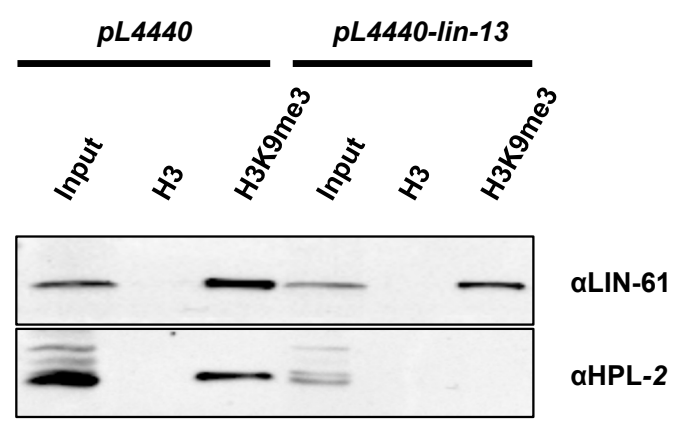

Figure 3.19: lin-13 RNAi does not affect LIN-61-H3K9me3 interaction

Peptide pull-down experiments were performed with either $\mathrm{H} 3$ unmodified or $\mathrm{H} 3 \mathrm{~K} 9 \mathrm{me} 3$ peptide using N2 worm lysates fed with either pL4440 or lin-13-pL4440 bacteria. Bound proteins were resolved on SDS-PAGE and detected by western blotting using anti-LIN61 (top panel) or anti-HPL-2 (bottom panel) antibodies. 


\subsection{3 lin-13 genetically interacts with hpl-2 and lin-61}

Since HPL-2, LIN-61 and LIN-13 interact to form a complex, we asked whether these three factors genetically interact during vulva development. We performed lin-13 RNAi assay in hpl-2 (tm1489) and lin-61 (tm2649) mutant worms. In our analyses, we also included two SynMuv A mutants namely lin-15a (n767) and lin-56 (n272) since lin-13 is a known SynMuv B gene (96). We also used mbtr-1 (n4775), another MBT-repeat containing protein similar to LIN-61 and met-2 (tm4256), a histone H3K9 methyltransferase as SynMuv B mutant.

Since N2 worms showed 29 \% muv phenotype upon lin-13 RNAi, we had to attenuate the RNAi feeding in order to access the possible genetic interactions. We therefore, omitted the IPTG from RNAi plates and relied on the leaky expression of the lac operon in order to drive the expression of dsRNA in bacteria. This attenuated the muv phenotype in N2 worms to $\sim 30-40 \%$ and allowed us to study possible genetic interaction of lin-13 with other genes. We performed attenuated RNAi in various mutants (figure 3.20) alongside N2 worms and scored for muv phenotype in F1 adults. As expected, we observed low penetrant muv phenotype in N2 worms. Moreover, both SynMuv A mutants lin-15a (n767) and lin-56 (n172) displayed a significantly higher $(\mathrm{P}<0.01$, ANOVA) percentage of muv animals, $73 \pm 10 \%$ and $81 \pm 19 \%$ respectively upon lin-13 RNAi (figure 3.20).

All hpl-2 (tm1489) and met-2 (tm4256) worms were sterile in first generation itself and did not give rise to any progeny. Hence, these could not be scored. We concluded that both $\mathrm{hpl}-2$ and met-2 show strong genetic interaction with lin-13. Interestingly, upon lin$13 \mathrm{RNAi}$, lin-61 worms also showed increased muv phenotype compared to N2 $(67 \pm 8 \%$, $\mathrm{P}<0.01)$. In contrast, mbtr-1 worms, upon lin-13 RNAi did not show any increase or decrease in muv phenotype under these conditions. This suggests that lin-13 genetically interacts specifically with lin-61 but not mbtr-1 (figure 3.20). 


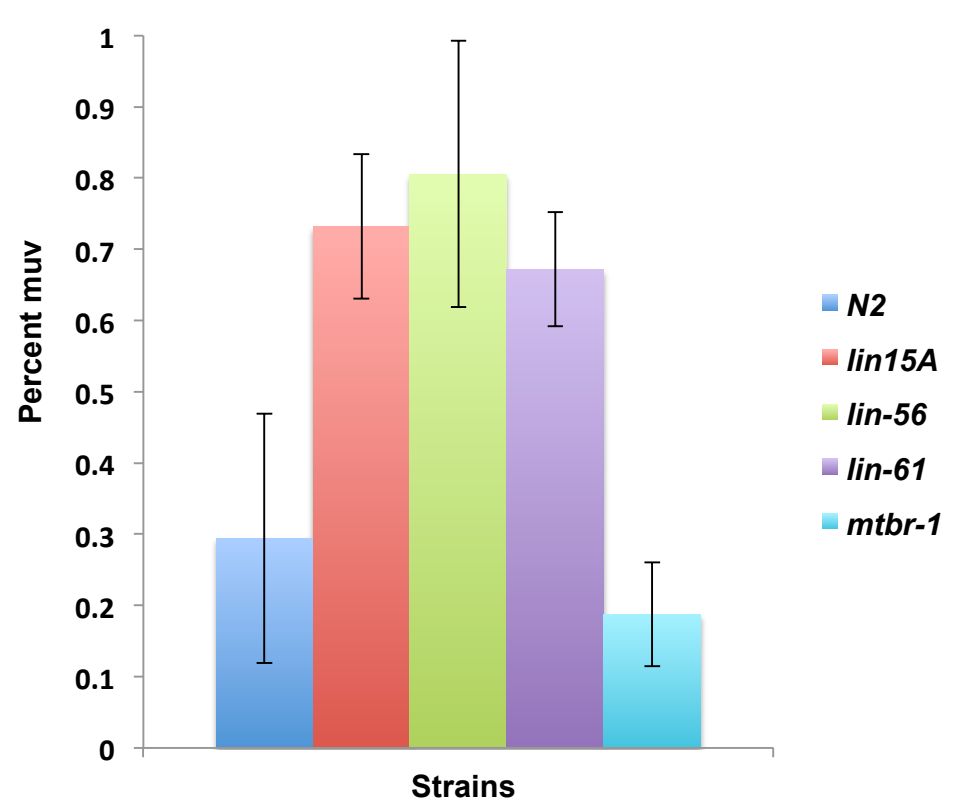

Figure 3.20: lin-13 genetically interacts with lin-61

N2 and other mutant strains (as shown in the graph) were fed with either pL4440 or lin13-pL4440 bacteria for two generations. F2 worms were scored for either WT or muv phenotype (worms with more than one vulval projections). Mean penetrance of muv phenotype was calculated from five independent experiments (Turkey's HSD test, oneway ANOVA, $P<0.01$, as denoted by *). Error bar represents \pm standard deviation. lin13 RNAi experiments were performed with Elizabeth Miller.

Our results suggested that HPL-2, LIN-61 and LIN-13 although, biochemically interact with each other, are not completely epistatic to each other in terms of their function(s) in vulva development.

Based on these findings, we hypothesized that HPL-2/LIN-61/LIN-13 form multiple complexes that bind distinct regions in chromatin and thereby regulate distinct subsets of genes including the genes associated with vulva development and fertility in $C$. elegans. Therefore, we resorted to a functional genomics approach and decided to perform gene expression analysis of hpl-2, lin-61 mutants and lin-13 RNAi worms to define transcriptional targets of these proteins. 
We also decided to identify the binding sites of these proteins on the $C$. elegans genome and then overlap these datasets with gene expression datasets to link chromatin association to transcription regulation by HPL-2/LIN-61/LIN-13 complexes in the regulation vulva development.

\subsection{HPL-2/LIN-61/LIN-13: functional genomics approach}

\subsubsection{Gene expression analysis of hpl-2, lin-61 and lin-13}

We carried out gene expression analysis in wild type and corresponding hpl-2, lin-61 mutant and lin-13 RNAi L3-staged worms grown at $20^{\circ} \mathrm{C}$, since vulval induction occurs during this stage.

\subsubsection{Microarray analysis of hpl-2 and lin-61 mutants}

We analyzed lin-61, hpl-2 and hpl-2; lin-61 double mutants with a goal to identify common genes that are regulated by HPL-2 and LIN-61. We performed microarray analysis of the RNA obtained from each of these mutants in comparison to N2 worms. We found differentially expressed genes for each mutant compared to N2 worms with stringent filters (FDR $<0.05$ and $\log _{2}$ [fold change] $>1.5$ ). Among the $\sim 16,000$ target probes covering C. elegans transcriptome that we tested, we found 1201, 1891 and 2723 misregulated genes in lin-61, hpl-2 and lin-61; hpl-2 mutants respectively as compared to $\mathrm{N} 2$ worms (figure 3.21A).

HPL-2 and LIN-61 are heterochromatin associated factors and presumably repress transcription of target genes (44). Accordingly, in all these mutants, more genes were found to be upregulated than downregulated. 771 of 1201,1174 of 1891 and 1552 of 2723 genes were upregulated in lin-61, hpl-2 and lin-61; hpl-2 mutants, respectively.

We overlapped lin-61_N2 dataset, $h p l-2 \_N 2$ dataset and lin-61; hpl-2_N2 dataset to identify the commonly regulated genes by HPL-2 and LIN-61. We found total 572 overlapping genes of which 444 genes were upregulated(Fig3.21A).

To identify the biological processes associated with the overlapping genes, we performed GO enrichment analysis of the 572 overlapping genes. 
Expectedly, we found strong enrichment for GO terms (figure 3.21B) such as 'negative regulation of developmental process', which includes genes regulated in vulva development $\left(P\right.$ - value $\left.=3.2 e^{-02}\right)$. Since, microarray data is not strictly quantitative, we verified expression of some of these genes using real time RT-PCR. For real time RTPCR analysis, we chose lin-13, lin-15b, pgl-1, prg-1, puf-1 genes, all of which are associated with vulva development and were upregulated in these mutants in our transcriptome analysis. We found that there was a general agreement of microarray data with real time RT-PCR data (figure 3.21C).

(A)

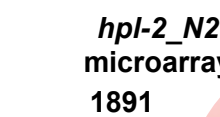

(444)

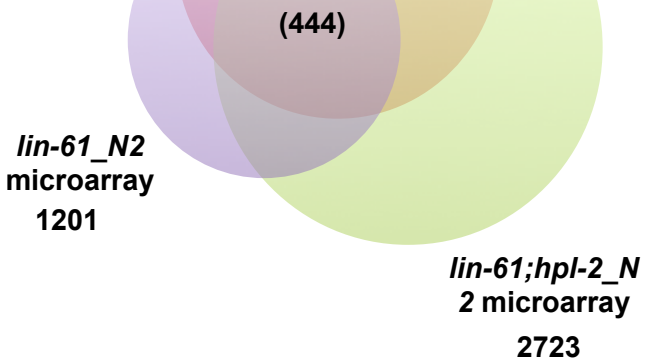

(B)

2723
(C)

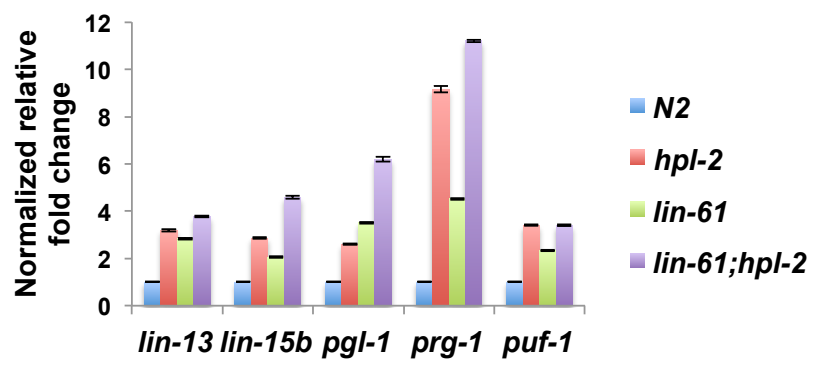

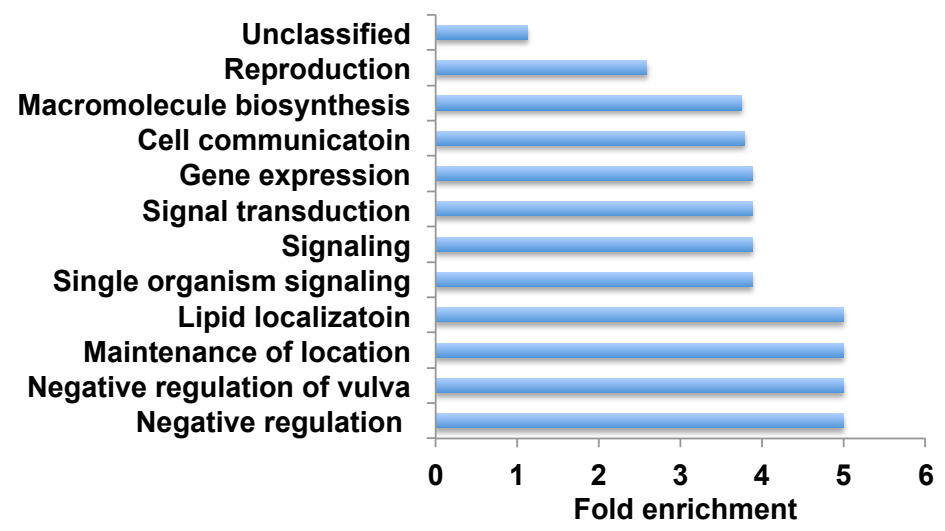

Figure 3.21: Transcriptome analysis in lin-61, hpl-2 and lin-61; hpl-2 mutants 
(A) Venn diagrams on the basis of microarray analysis showing the extent of overlap among the genes misregulated in lin-61, hpl-2 or lin-61; hpl-2 mutant. Values for fold change are from 3 biological replicates (FDR <0.05, [ $\log _{2}$ (fold change)] >1.5). (B) Bar graph showing a GO enrichment analysis of the overlapping genes among all three microarray datasets. (C) Real time RT-PCR validation of transcriptome datasets for genes that are implicated in vulva development. Relative fold change (normalized to housekeeping gene, rpl-26) for lin-13, lin-15b, pgl-1, prg-1 and puf-1 in hpl-2, lin-61 and lin-61; hpl-2 mutants. Fold change represents 3 biological replicates. Error bars represent \pm SEM.

\subsubsection{RNA-seq analysis upon lin-13 RNAi}

Our next goal was to identify the commonly regulated genes by LIN-13, HPL-2 and LIN61 We performed RNA-seq analysis in lin-13 RNAi animals compared to pL4440 RNAi N2 animals.
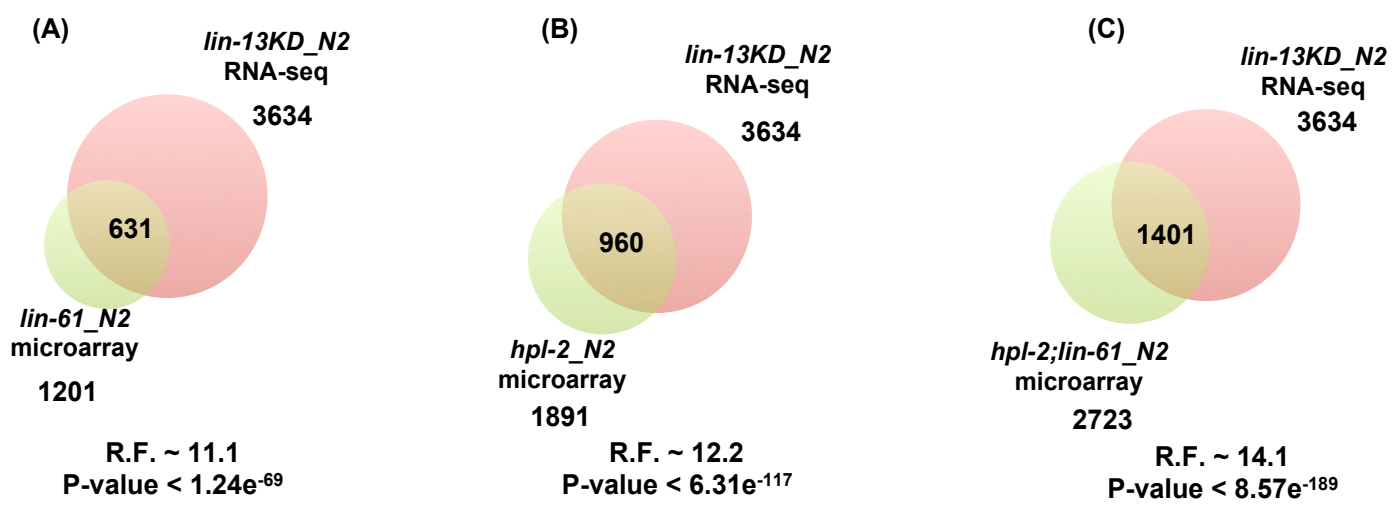

(D)

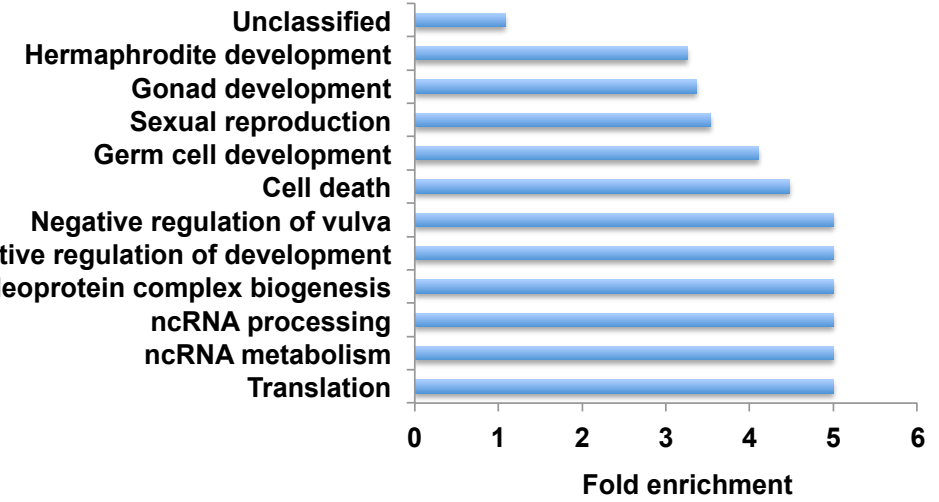

Figure 3.22: RNA-sequencing analysis upon lin-13 RNAi in C. elegans 
Venn diagrams $(A, B, C)$ on the basis of RNA-seq analysis and transcriptome analysis showing the extent of overlap between lin-13 and (A) lin-61, (B) hpl-2, (C) lin-61; hpl-2 datasets. Values for fold change are from 3 biological replicates (FDR $<0.05$, $\left[\log _{2}\right.$ (fold change)] >1.5). (D) Bar graph showing a GO enrichment analysis of the overlapping genes between lin-13KD_N2 and lin-61;hpl-2_N2 datasets. P-values and R.F. values were determined using hyper-geometric tests. RNA-seq analysis of lin-13 RNAi was performed by Dr. Vincenzo Capace and Dr. Stefan Bonn.

We found that lin-13 RNAi resulted in misregulation of 3634 genes. Of these, 2357 were upregulated and 1227 were downregulated. We overlapped these genes with hpl-2_N2 or lin-61_N2 or hpl-2; lin-61_N2 microarray datasets and found 631, 960 and 1401 overlapping genes (figure 3.22A, B, C). GO enrichment analysis of the 1401 genes that overlapped between lin-13KD_N2 and lin-61;hpl-2_N2 datasets showed strong enrichment for genes associated with vulva development and reproduction (figure 3.20D). We also observed strong enrichment of the genes involved in ncRNA processing and ncRNA biogenesis suggesting the involvement of these factors in RNAi pathways.

We conclude that HPL-2, LIN-61 and LIN-13 commonly regulate transcription of several genes associated with vulva development. In order to find the direct transcriptional targets of these factors, we analyzed the binding sites of these factors on the $C$. elegans genome.

\subsubsection{Identification and functional characterization of HPL-2/LIN-61/LIN-13 binding sites on C. elegans genome}

\subsubsection{Identification of HPL-2/LIN-61/LIN-13 binding sites}

modENCODE is a research network aimed to provide a comprehensive encyclopedia of functional genomic elements in D. melanogaster and C. elegans (118). modENCODE consortium has identified genome-wide binding sites for several transcription factors, chromatin modifications and chromatin proteins in C. elegans (119, 120). During the course of our study, the ChIP-seq datasets for HPL-2, LIN-13 and LIN-61 became available. We selected these ChIP-seq datasets of H3K9me3, HPL-2, LIN-61 and LIN- 
13 (table 2.13) from modENCODE server, designed the analysis pipeline (2.5.1) and extracted the binding sites for H3K9me3, LIN-61, HPL-2 and LIN-13.

We analyzed individual binding sites of HPL-2, LIN-61, and LIN-13. We found that HPL2 shows two distinct types of distribution. In one case, HPL-2 binding sites were sharp and concentrated in promoter regions whereas in other case, HPL-2 was broadly distributed over the body of genes especially introns. LIN-61 mainly had broad distribution over gene bodies with occasional sharp peaks. LIN-13 on the other hand, mostly had sharp, localized peaks corresponding to promoters and transcription start sites.

\subsubsection{Functional characterizarion of HPL-2/LIN-61/LIN-13 binding sites}

In order to identify the genes directly regulated by HPL-2/LIN-61/LIN-13, we overlapped ChIP-seq and transcriptome/RNA-seq datasets. We identified 307 genes that are bound by HPL-2 and are misregulated in the hpl-2 mutant (figure 3.23A). Similarly, we identified 163 and 209 genes that are bound by LIN-61 and LIN-13 that were misregulated in lin-61 mutant and lin-13 RNAi worms respectively (figure 3.23B, C). Among the genes that were bound by these factors, the number of upregulated genes was much higher than the number of downregulated genes in all the mutant worms (figure 3.23.D, E, F). 


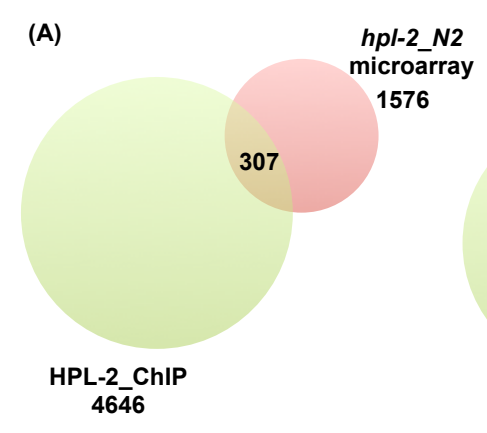

(D)

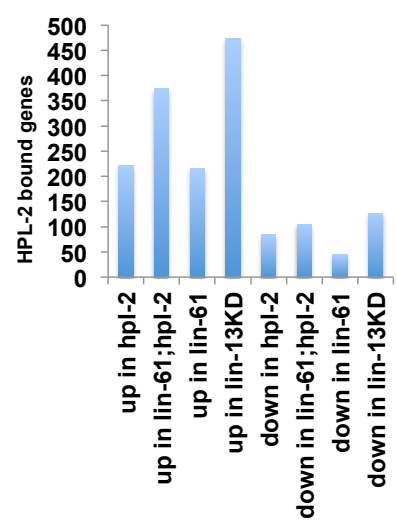

(B)

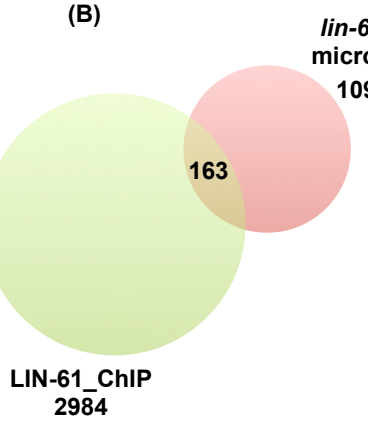

(E)

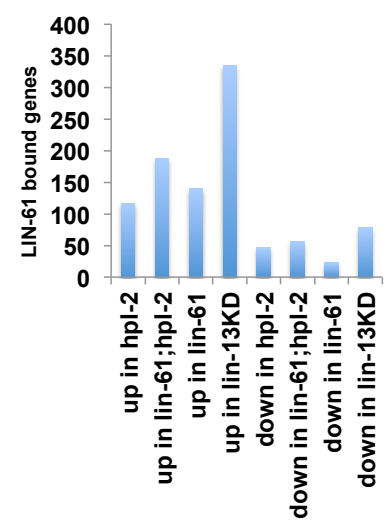

(C)

lin-13KD_N2 RNA-seq

3635

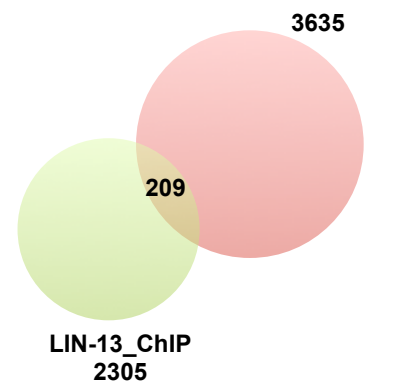

(F)

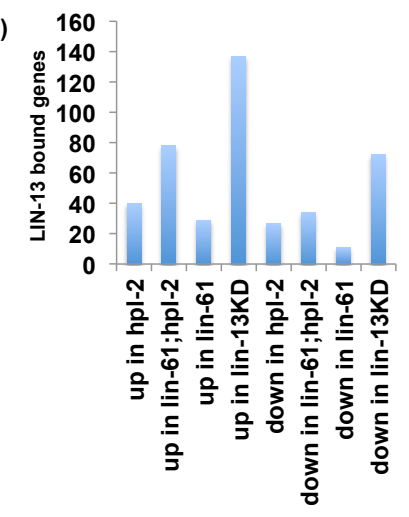

Figure 3.23: Direct targets of HPL-2, LIN-61 and LIN-13

Overlap of ChIP-seq datasets with transcriptome/RNA-seq datasets (A) for HPL-2, HPL2_ChIP-seq dataset was overlapped with $h p l-2 \_N 2$ microarray (B) for LIN-61, LIN61_ChIP-seq dataset was overlapped with lin-61_N2 microarray (C) for LIN-13, LIN13_ChIP-seq dataset was overlapped with lin-13KD_N2 RNA-seq dataset. The graphs showing either HPL-2-bound (D), LIN-61-bound (E) or LIN-13-bound (F) genes that are misregulated in hpl-2, lin-61, hpl-2;lin-61 and lin-13KD worms.

HP1 proteins are also reported to regulate gene expression positively (67). Consistently, we found a subset of HPL-2 bound genes that are positively regulated by HPL-2 (figure 3.23D). We also found that LIN-61 and LIN-13 positively regulate at least some of the genes that they bind (figure 3.23E, F). 


\subsubsection{Functional characterization of distinct HPL-2/LIN-61/LIN-13 complexes}

Based on our biochemical analysis of HPL-2, LIN-61 and LIN-13, we hypothesized that these proteins form distinct complexes. We rationalized that these complexes bind distinct regions in the genome and regulate distinct subsets of genes. In order to identify the binding sites of these distinct complexes formed by HPL-2, LIN-61 and LIN-13, we overlapped ChIP-seq datasets of these factors.

\subsubsection{H3K9me3-dependent HPL-2/LIN-61/LIN-13 complexes}

Based on our earlier results, we hypothesized that $\mathrm{H} 3 \mathrm{~K} 9 \mathrm{me} 3$ enriched regions in the genome recruit LIN-61/HPL-2/LIN-13 proteins in an interdependent manner. In order to identify such $\mathrm{H} 3 \mathrm{~K} 9 \mathrm{me} 3$ enriched regions that are co-occupied by these three factors, we overlapped ChIP peaks for H3K9me3, HPL-2, LIN-61 and LIN-13. Surprisingly, we found only 20 peaks over the entire $C$. elegans genome where all these factors are cooccupied (figure 3.24). Interestingly, these peaks corresponded to germline RNAi pathways such as piwi and 21U-RNA genes.

Next, we hypothesized that H3K9me3 recruits LIN-61 and HPL-2 but not LIN-13, containing sub-complex. We therefore, sorted ChIP peaks containing only H3K9me3, LIN-61 and HPL-2 but not LIN-13 and found 115 overlapping peaks where H3K9me3, LIN-61 and HPL-2 are co-occupied (figure 3.24). We also sorted peaks that are cooccupied with only H3K9me3 and LIN-61 as well as only H3K9me3 and HPL-2. Unexpectedly, we found only 19 peaks corresponding to H3K9me3-LIN-61 while we found 390 peaks corresponding to H3K9me3-HPL-2 (figure 3.24).

This is in contrast to our biochemical analysis (section 1.2,1.3) where we found that HPL-2 depends on LIN-61 for its association with H3K9me3. HPL-2 co-occupies with H3K9me3 most strongly of all the three factors suggesting the involvement of unknown mechanism(s) that could recruit HPL-2 to H3K9me3 enriched region 


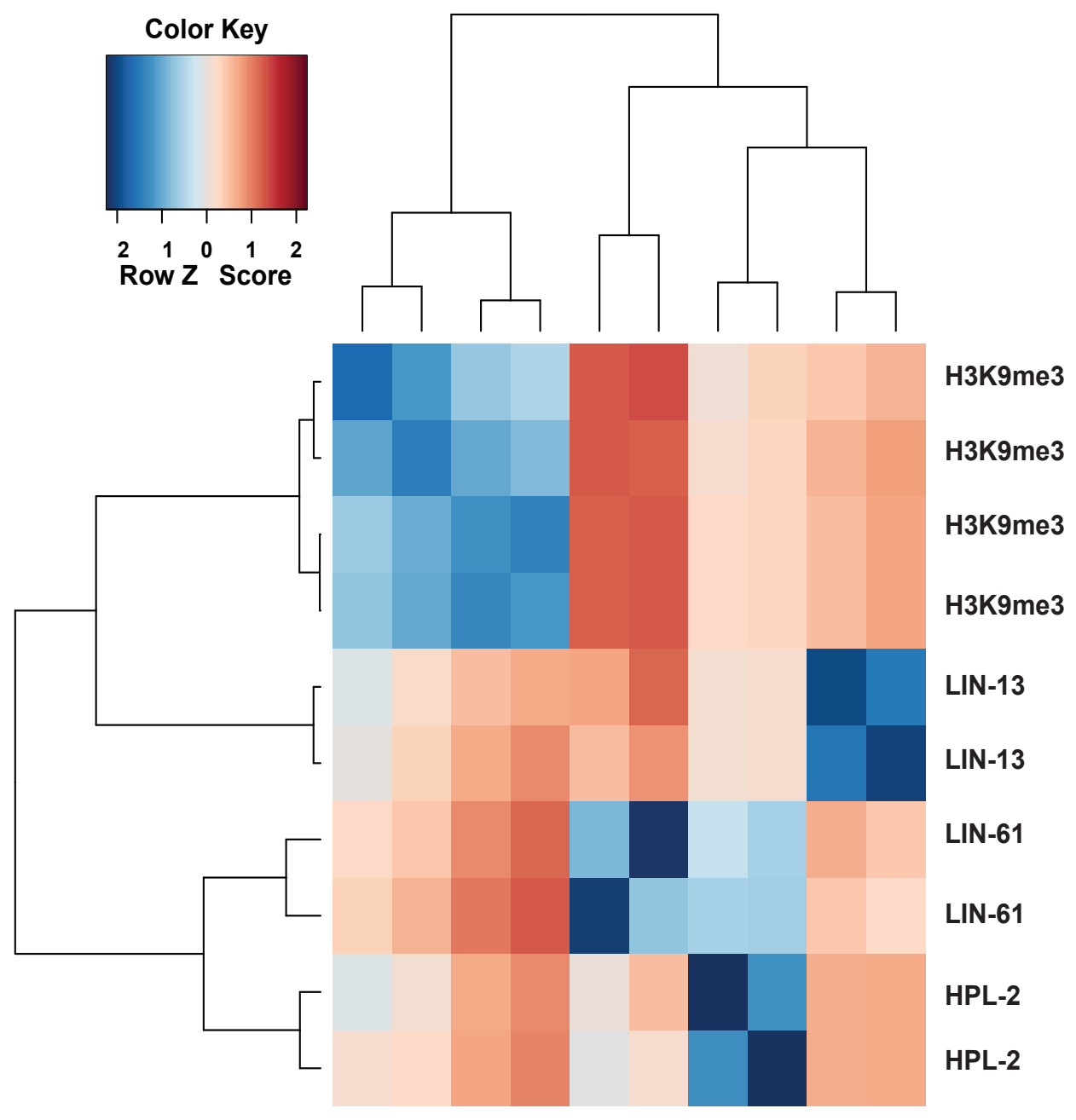

Figure 3.24: ChIP-seq analysis of modENCODE datasets for H3K9me3, HPL-2, LIN-61 and LIN-13

The heatmap was generated using MEDIPS package in $\mathrm{R}$, which involved counting the number of reads in bins of $100 \mathrm{bp}$, calculating the correlations between the samples, and the heatmap was plotted with a regular heatmap function. ChIP-seq analysis of modENCODE datasets was performed by Orr Shormoni and Dr. Stefan Bonn. 


\subsubsection{H3K9me3-independent genome wide distribution of the HPL-2/LIN-61/LIN-}

\section{3 complexes}

We searched for the overlaps among HPL-2, LIN-61 and LIN-13 independent of H3K9me3. We applied hyper-geometric statistics to find the significance of their cooccupancy. We found that all three factors are co-occupied at 831 unique peaks (figure 3.25A, D). This suggests that the HPL-2/LIN-61/LIN-13 complex primarily gets recruited to chromatin in $\mathrm{H} 3 \mathrm{~K} 9 \mathrm{me} 3$ independent manner.

Strikingly, we found that HPL-2 and LIN-61 but not LIN-13 were co-occupied at 4822 peaks over the genome (figure 3.25A, B). This represents $65 \%$ and $85 \%$ of all HPL-2 peaks and LIN-61 peaks over the genome respectively. These are unique peaks containing only HPL-2 and LIN-61 and does not contain LIN-13 and/or H3K9me3 peaks. Interestingly, HPL-2 was also co-occupied at 581 peaks with LIN-13 but not LIN-61 and/or H3K9me3 (figure 3.25A, C).

(A)

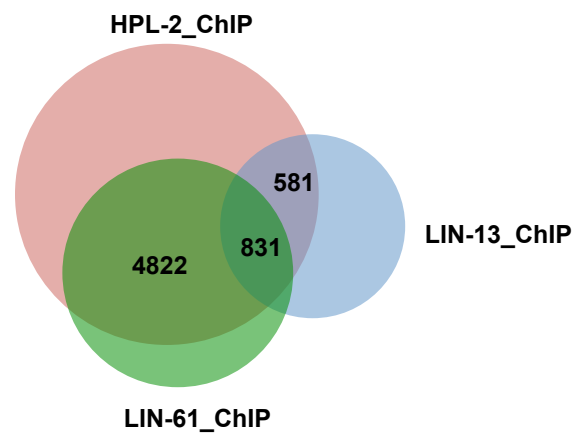

(B) Complex I

(C) Complex II

(D) Complex III
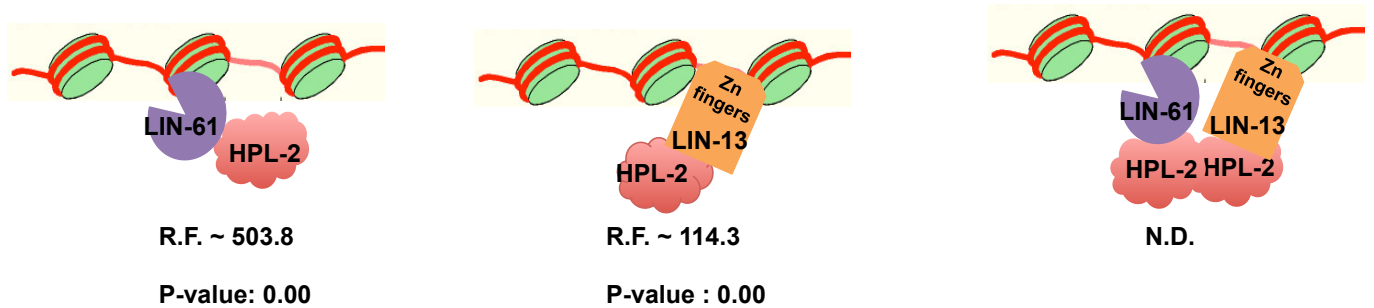

P-value : 0.00

Figure 3.25: Statistically ranked models that could exist among HPL-2, LIN-61 and LIN-13 based on the abundance of their co-occupancy 
(A) Venn diagram representing overlaps of HPL-2, LIN-61 and LIN-13 ChIP peaks. (B) Complex I: The protein complex formed by HPL-2 and LIN-61 based on the enrichment of its binding sites. (C) Complex II: The protein complex formed by HPL-2 and LIN-13 based on the enrichment of its binding sites. (D) Complex III: The protein complex formed by HPL-2, LIN-61 and LIN-13 based on the enrichment of its binding sites. Representation factor (R.F.) and P-value generated by hyper-geometric test.

\subsubsection{HPL-2/LIN-61/LIN-13 complexes regulate distinct subsets of target genes}

We studied the binding sites of the proposed three (figure 3.25) distinct HPL-2/LIN61/LIN-13 complexes for their effect on transcription regulation of genes where they reside. For HPL-2-LIN-61 complex, we had earlier found 4822 binding sites on the $C$. elegans genome (section 3.7.3.2). We overlapped the subset of genes associated with these binding sites with either hpl-2_N2 or lin-61_N2 microarray datasets. We found that 146 genes are misregulated in the $h p l-2$ mutant while 114 genes are misregulated in the lin-61 mutant, where both HPL-2 and LIN-61 are co-occupied (figure 3.26A). This suggests that loss of $h p l-2$ has a more pronounced effect on the genes regulated by the HPL-2-LIN-61 complex. Interestingly, 73 genes are misregulated by loss of either HPL-2 or LIN-61 (figure 3.26A). For HPL-2-LIN-13 complex, we had found 581 binding sites. We overlapped the subset of genes associated with these binding sites with either hpl-2_N2 or lin-13kd_N2 microarray/RNA-seq datasets. We found that 29 genes are misregulated in lin-13 mutant while 11 genes are misregulated in hpl-2 mutant of the 494 genes bound by HPL-2-LIN13 complex (figure 3.26B). For HPL-2-LIN-61-LIN-13 complex, we had found 831 binding sites (section 3.7.3.2). We overlapped the subset of genes associated with these binding sites with hpl-2_N2, lin-61_N2 or lin-13kd_N2 microarray/RNA-seq datasets. We found that of the 557 genes bound by this complex, 80 genes were misregulated upon lin-13 RNAi (figure 3.26C). Loss of LIN13 had the most pronounced effect on the genes regulated by this complex. 


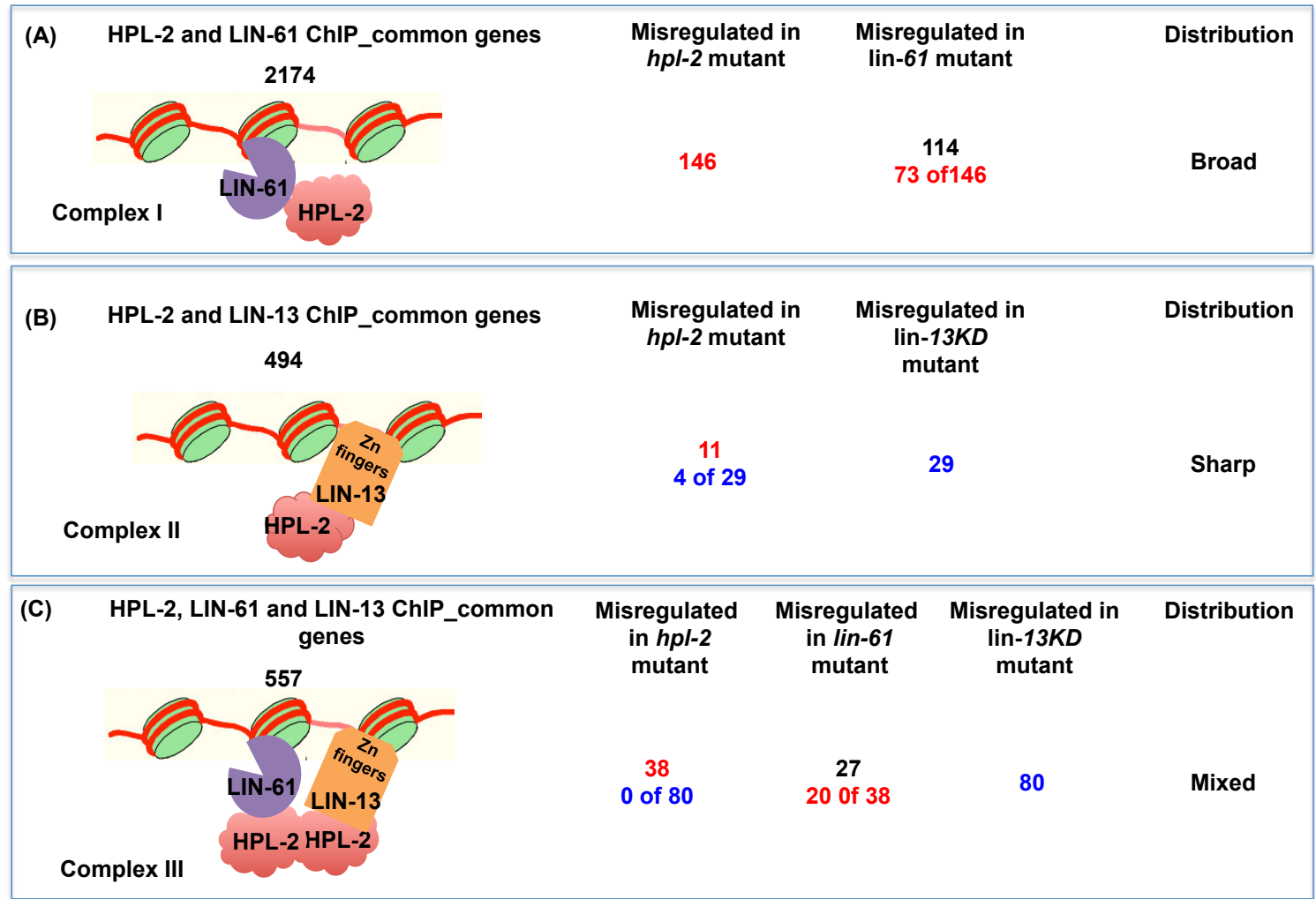

Figure 3.26: Direct targets of three distinct complexes of HPL-2/LIN-61/LIN-13

(A) Hypothetical LIN-61-HPL-2 complex (complex I): the genes bound by this complex, the genes that are misregulated in $h p l-2$ and lin-61 mutants, the characteristics of the binding sites. (B) Hypothetical LIN-13-HPL-2 complex (complex II): the genes bound by this complex, the genes that are misregulated in hpl-2 and lin-13 knock-down worms, the characteristics of the binding sites. (C) Hypothetical HPL-2-LIN-61-LIN-13 complex (complex II): the genes bound by this complex, the genes that are misregulated in $h p /-2$, lin-61 and lin-13 knock-down worms, the characteristics of the binding sites. Numbers in red, blue and black denotes genes misregulated in hpl-2, lin-13 RNAi and lin-61 mutants respectively.

Interestingly, we found that the binding sites of HPL-2-LIN-61 complex had different characteristics compared to the binding sites of HPL-2-LIN-13 or HPL-2-LIN-61-LIN-13 complex. 


\subsubsection{Broad distribution of HPL-2-LIN-61 complex}

The binding sites that are co-occupied only by HPL-2 and LIN-61 had a broad distribution (Figure 3.27A). These binding sites ranged from $2 \mathrm{~kb}-10 \mathrm{~kb}$, sometimes even spanning more than $20 \mathrm{~kb}$, covering entire genes. These binding sites mainly consisted of intronic regions of the genes, which then spread on both sides, covering promoter-transcription start sites on one side, while covering transcription termination site on the other side. We found that of the total 4822 binding sites where HPL-2 and LIN-61 were co-occupied, 3639 binding sites (75\% of all binding sites) formed broad domains covering around 990 genes. We also found 466 sharp binding sites ( $9 \%$ of all binding sites) formed by HPL-2 and LIN-61 in promoter regions suggesting that they might associate with other transcription factors as well.

\subsubsection{Sharp distribution of HPL-2-LIN-13 complex}

We found that the binding sites that are co-occupied only by HPL-2 and LIN-13 had a sharp distribution (Figure 3.27B). These binding sites were mainly located in promoter regions. Of the total 581 binding sites that are co-occupied by HPL-2 and LIN-13, 564 binding sites (97\%) had sharp distribution whereas only 17 binding sites had broad distribution. Transcription factors mainly get recruited in promoter regions and recognize a specific sequence motif, and therefore have sharp distribution in ChIP-seq studies (216). However, so far no transcription factor activity has been reported for LIN-13. These binding sites ranged from $200 \mathrm{bp}-1000 \mathrm{bp}$, mostly concentrated in the promoter regions, occasionally covering gene bodies.

\subsubsection{Bimodal distribution of HPL-2-LIN13-LIN-61 complexes}

We found that the binding sites that are co-occupied by HPL-2, LIN-61 and LIN-13 had sharp distribution at some binding sites and broad distribution at others (figure 3.27B). We found that of the total 831 binding sites, 418 binding sites (50.4\%) formed broad domains covering 206 genes. These binding sites were mainly located in gene bodies. The remaining 413 binding sites $(49.6 \%)$ had sharp distribution. These binding sites were mainly located in promoter regions. 
(A)

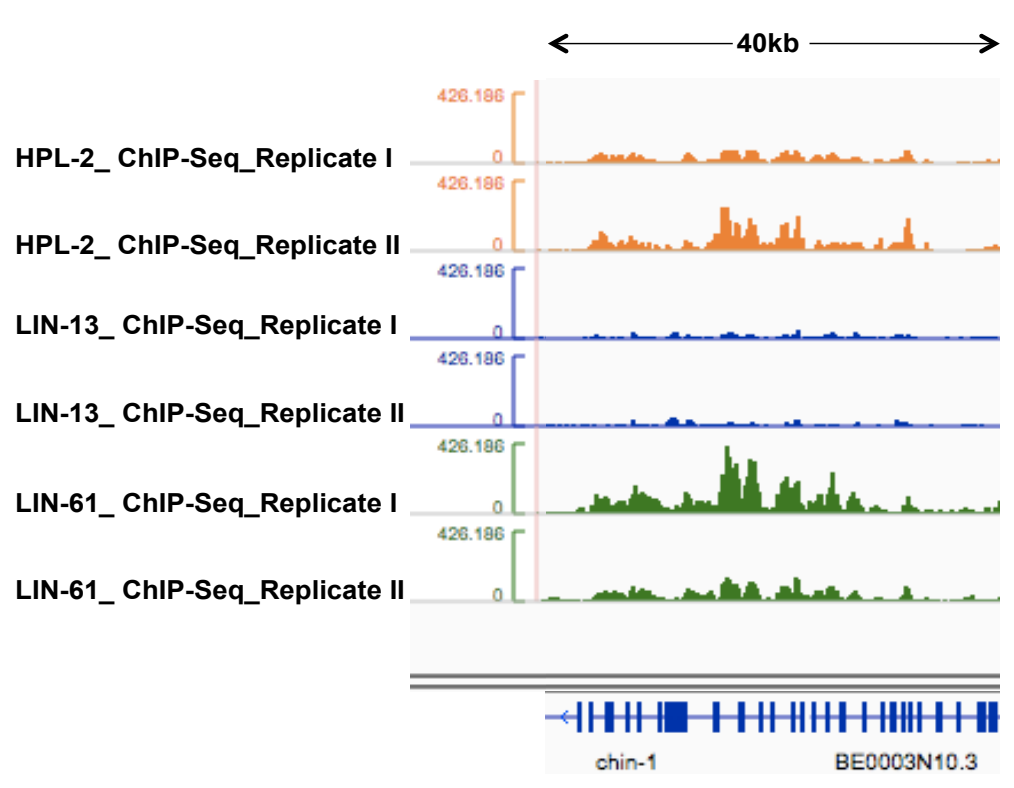

(B)

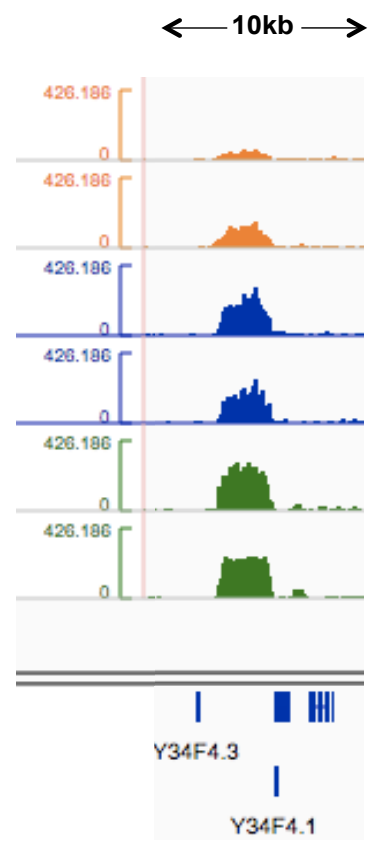

Figure 3.27: Distinct distribution HPL-2/LIN-61/LIN-13 complexes

Screenshot of the ChIP-seq peaks of HPL-2, LIN-13 and LIN-61 showing (A) broad peaks of HPL-2-LIN-61 complex spanning chin-1 and BEN0003N10.3 and (B) sharp peaks of HPL-2-LIN-13-LIN-61 complex enriched at promoters of Y34F4.3 and Y34F4.1.

Taken together, our data suggests that apart from LIN-61, HPL-2 and LIN-13 forming a complex, HPL-2 also forms sub-complexes with LIN-61 and LIN-13, all of which are recruited to chromatin via distinct mechanisms. The nature of the binding sites suggests that at some regions, the recruitment occurs in a transcription factor dependent manner (sharp peaks). In other cases, where recruitment occurs mostly in introns, it could be transcription factor independent. Intronic RNAs are known to recruit PRC2 complex to chromatin via their association with EZH2 (196). In mammals, immunofluorescence analysis of cells with RNase treatment before fixation has shown loss of HP1 from pericentric heterochromatic foci (64). Also, studies have shown that ncRNAs might play an important role in HP1 recruitment to chromatin $(63,121)$. HPL-2 has also been recently implicated in RNAi pathways $(84,85,122)$. 
Based on these considerations, we hypothesized that recruitment of HPL-2-LIN-61 complex in the intronic regions could occur in an RNA dependent manner. We therefore, tested putative role of RNA in modulation of HPL-2/LIN-61/LIN-13 complexes.

\subsection{RNase-sensitivity of HPL-2/LIN-61/LIN-13 complexes}

We treated C. elegans nuclear extracts with RNase $A$ and performed peptide pull-down assay. We also included DNase treated extracts as controls. We found that upon, RNaseA treatment, HPL-2 recovery on H3K9me3 peptide was lost whereas LIN-61 association to $\mathrm{H} 3 \mathrm{~K} 9 \mathrm{me} 3$ peptide remained unaffected (figure $3.28 \mathrm{~A}$ ). We did not observe any loss of HPL-2 or LIN-61 binding to H3K9me3 upon DNase treatment suggesting the specific involvement of RNA. As another control, we performed a similar experiment using $h p l-1:: g f p$ extract and we did not see any loss of HPL-1 binding to $\mathrm{H} 3 \mathrm{~K} 9 \mathrm{me} 3$ upon RNase A treatment (figure 3.28B) suggesting that RNA-association is specific to HPL-2. 


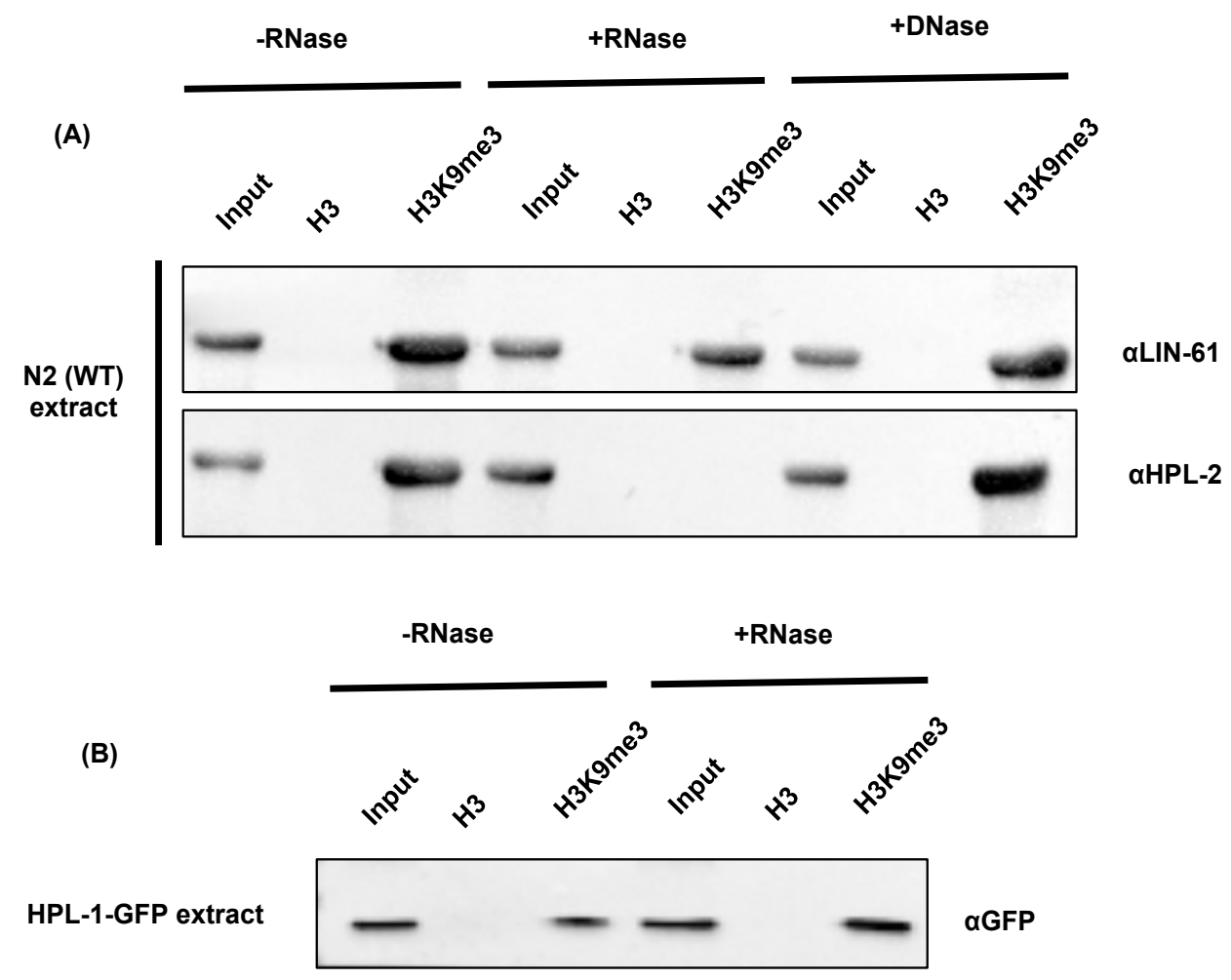

Figure 3.28: HPL-2/LIN-61/LIN-13 complexes get disrupted upon RNase treatment

Peptide pull-down assay was performed with $\mathrm{H} 3$ unmodified or $\mathrm{H} 3 \mathrm{~K} 9 \mathrm{me} 3$ peptide using either N2 extract. Bound proteins were separated using SDS-PAGE and detected by western blotting using anti LIN-61 (top panel) or anti HPL-2 antibodies (bottom panel). (B) Peptide pull-down assay was performed with $\mathrm{H} 3$ unmodified or $\mathrm{H} 3 \mathrm{~K} 9 \mathrm{me} 3$ peptide using either $h p l-1:: g f p$ extract. Bound proteins were separated using SDS-PAGE and detected by western blotting using anti-GFP antibodies.

In order to test whether loss of HPL-2 association with $\mathrm{H} 3 \mathrm{~K} 9 \mathrm{me} 3$ is indeed due to loss of HPL-2-LIN-61 interaction, we performed immunoprecipitation assays using extracts from $h p l-2:: g f p$ worms. We used either $-/+$ RNase A treated extracts to set up immunoprecipitation assays or performed immunoprecipitation first and then /+RNase A treated the beads. Both these methods are targeted to digest all possible RNA 
species that might stabilize HPL-2/LIN-61/LIN-13 complexes. As can be seen in figure 3.29A and 3.29B, upon RNase A treatment, binding of HPL-2 to LIN-61 and LIN-13 is lost.

(A)

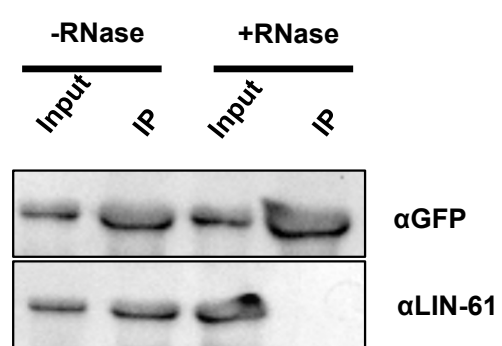

HPL-2 GFP extract treated with -/+ RNase
(B)

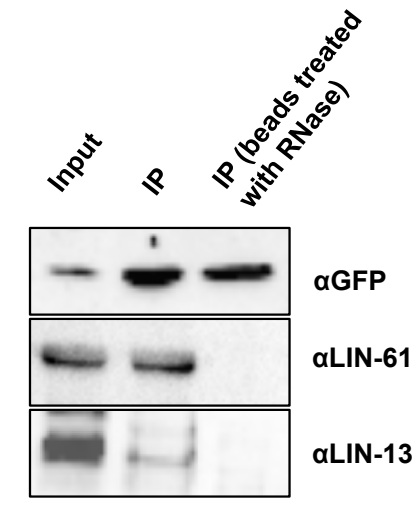

HPL-2 GFP beads (after IP) treated with -/+ RNase

Figure 3.29: HPL-2/LIN-61/LIN-13 complexes get disrupted upon RNase treatment

GFP immunoprecipitation was performed with GFP_Trap using $h p /-2:: g f p$ extract. (A) Immunopreciptation was performed after -/+ RNase A treatment of hpl-2::gfp extract. Bound proteins were detected by western blotting using anti-GFP (top panel) or anti LIN-61 antibodies (bottom panel). (B) Immunoprecipitation was performed and subsequently, beads were treated with -/+ RNase A. Bound proteins were detected by western blotting using anti-GFP (top panel) or anti LIN-61 antibodies (middle panel) or anti LIN-13 antibodies (bottom panel).

Taken together, our data suggests that HPL-2, LIN-61 and LIN-13 form multiple RNAdependent complexes that bind distinct regions in chromatin and regulate distinct subsets of target genes including genes associated with vulva development. 


\section{Discussion}

Heterochromatin protein 1 and its association with $\mathrm{H} 3 \mathrm{~K} 9 \mathrm{me} 3$ are required for heterochromatin formation and transcription regulation (203). However, molecular function(s) of HP1 proteins and mode of their association with chromatin are unclear. HP1 family proteins are known to associate with $\mathrm{H} 3 \mathrm{~K} 9 \mathrm{me} 3$ in vitro. However, their binding to chromatin does not completely correlate with $\mathrm{H} 3 \mathrm{~K} 9 \mathrm{me} 3$ distribution in vivo. HPL-2, one of the two HP1 homologues in C. elegans is required for vulva development (104). However, its mechanism of recruitment to chromatin and molecular function(s) are unknown.

We provide evidence that HPL-2 does not associate directly with H3K9me3. Rather, it depends on LIN-61 for its association with H3K9me3. HPL-2 and LIN-61 biochemically interact with each other and their association with $\mathrm{H} 3 \mathrm{~K} 9 \mathrm{me} 3$ is crucial for vulva development. HPL-2 association with LIN-61 relies on its CSD, by which it also associates with LIN-13. Full length HPL-2 and possibly its multimerization are required for HPL-2-LIN-61-LIN-13 complex formation. Furthermore, HPL-2 and LIN-13 interaction is reduced in the absence of LIN-61 while the interaction between LIN-61 and LIN-13 is lost in the absence of HPL-2. We propose that HPL-2, LIN-61 and LIN-13 form multiple complexes that regulate transcription of distinct subsets of genes. In agreement, $h p l-2$, lin-61 and lin-13 genetically interact with each other during vulva development. Genome-wide distribution studies of these factors provide another evidence for the existence of multiple complexes among HPL-2, LIN-61 and LIN-13. These complexes are recruited to chromatin via distinct mechanisms and regulate distinct subsets of target genes associated with vulva development and fertility in C. elegans. Lastly, we provide evidence for a possible involvement of RNA in HPL-2/LIN-61/LIN-13 complexes and possibly play a role in RNAi mediated gene silencing in C. elegans. 


\subsection{HP1-H3K9me3 interaction in C. elegans}

\subsubsection{HPL-2 does not interact with H3K9me3 in vivo}

We found that HPL-2 associates with H3K9me3 peptide in peptide pull-down assays using $C$. elegans extracts. However, it does not interact with $\mathrm{H} 3 \mathrm{~K} 9 \mathrm{me} 3$ directly but depends on LIN-61. LIN-61, an MBT protein has been shown to directly bind H3K9me3 (97). We show that HPL-2 associates with LIN-61, which in turn directly binds H3K9me3 via its MBT repeat domains. We propose that LIN-61 regulates the recruitment of HPL-2 in $\mathrm{H} 3 \mathrm{~K} 9 \mathrm{me} 3$ enriched regions on chromatin in vivo. However, it is intriguing that HPL-2, a C. elegans HP1 homologue does not interact with $\mathrm{H} 3 \mathrm{~K} 9 \mathrm{me} 3$ directly.

\subsubsection{HPL-2 is a bonafide HP1 protein}

We studied the interaction of recombinant HPL-2 with H3K9me3 peptides in vitro. We found that HPL-2 binds $\mathrm{H} 3 \mathrm{~K} 9 \mathrm{me} 3$ peptide specifically with a binding strength that is in agreement with HP1-H3K9me3 interactions in flies and mammals $(30,56,124,125)$. Similar to other HP1 proteins, HPL-2 also binds H3K9me2 and H3K9me1 with intermediate strengths. Sequence alignment of HPL-2 with other HP1 proteins shows the conservation of critical residues that interact with $\mathrm{H} 3 \mathrm{~K} 9 \mathrm{me} 3$ including the aromatic amino acids that form the hydrophobic pocket in which the methylated lysine fits in. This suggests that HPL-2 is a bonafide HP1 family protein. However, our observation that HPL-2 does not interact directly with $\mathrm{H} 3 \mathrm{~K} 9 \mathrm{me} 3$ in extracts unravels the existence of negative regulation of HPL-2-H3K9me3 interaction in C. elegans.

In mammals, although all three isoforms of HP1 are known to bind H3K9me3 in vitro, their in vivo association to chromatin is not completely dependent upon H3K9me3 (123). HP1 $\alpha$ and HP1 $\beta$ are localized to H3K9me3 enriched pericentric heterochromatin while HP1Y is mainly localized to euchromatic regions (123). Phosphorylation of HP1Y is known to abolish its ability to interact with H3K9me3 (133). Similarly, phosphorylation of HP1 $\beta$ leads to disruption of its association with H3K9me3 and heterochromatin (132). We propose that negative regulation of HPL-2-H3K9me3 interaction is mediated by either non-H3K9me3 association of HPL-2, allosteric regulation or post-translational modification(s) of HPL-2 (figure 4.1). 


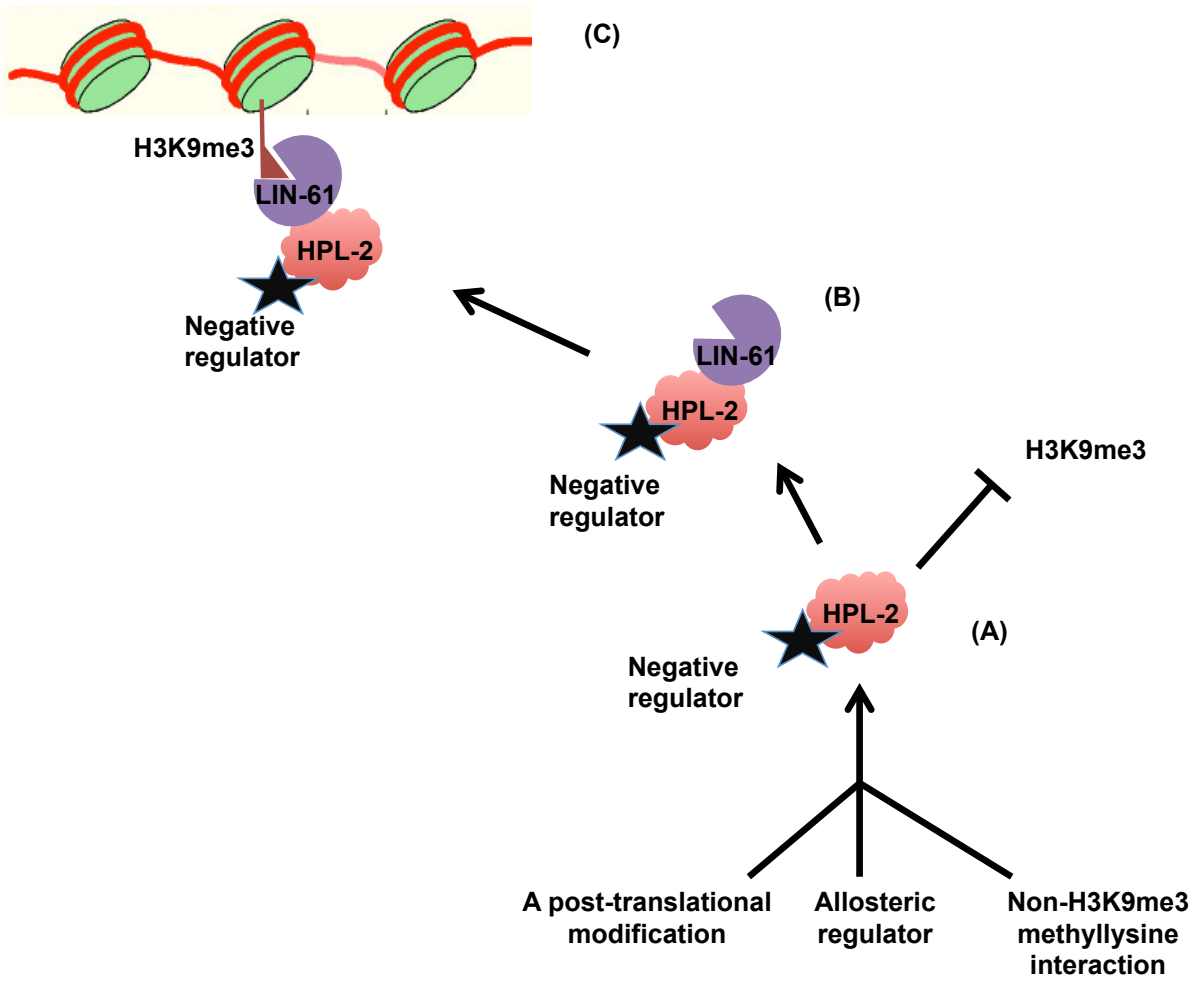

Figure 4.1: Negative regulation of HPL-2-H3K9me3 interaction in C. elegans

(A) HPL-2 is negatively regulated by, either a post-translational modification, allosteric regulation, non-H3K9me3 methyllysine interaction or a combination of these. This leads to loss of its ability to bind H3K9me3 directly (B) HPL-2 interacts with LIN-61. (C) LIN-61 interacts with $\mathrm{H} 3 \mathrm{~K} 9 \mathrm{me} 3$ and thereby recruits HPL-2 to chromatin. 


\subsubsection{HPL-2 might engage with non-histone methyllysine or in protein-protein interaction via its $C D$}

We hypothesize that HPL-2 CD might be engaged either with other methylated lysine(s) (either chromatin modification or modification in a chromatin associated protein) or another protein via protein-protein interaction (figure 4.1). Apart from H3K9me3, HP1 $\mathrm{CD}$ is known to bind methyllysines of other proteins. The examples of such interactions include recognition of $\mathrm{H} 1.4 \mathrm{~K} 26 \mathrm{me} 1, \mathrm{G} 9 \mathrm{aK} 185 \mathrm{me} 1$ and pRbK873me1 by HP1 proteins (31). Interestingly, it has also been reported that HPL-2 recovers better on H3K27me3 peptides than H3K9me3 in the extract (128). However, our competitive depletion assay, in which, we depleted the hypothetical competitive negative factor that inhibits HPL-2H3K9me3 interaction, did not restore HPL2-H3K9me3 interaction.

If at all, such a mode of negative regulation exists, it is inevitable to test HPL-2H3K9me3 interaction in the mutant background of all lysine methyltransferases. There are 38 putative KMT genes encoded in the C. elegans genome (129). If at all, HPL-2 engages in a non-histone methyllysine interaction, a particular methyltransferase mutant might lack this methyllysine and HPL-2 CD would be available for interaction with H3K9me3 peptides.

\subsubsection{Allosteric regulation of HPL-2-H3K9me3 interaction}

We hypothesize that HPL-2 associates with an allosteric factor (protein, RNA, small molecule) that negatively regulates HPL-2-H3K9me3 interaction (figure 4.1). HP1 proteins associate with several proteins via their CSD. However, till date no protein has been shown to allosterically regulate HP1-H3K9me3 interaction. In yeast, SWI6 interacts with heterochromatin transcripts via its hinge region. This leads to a conformational change in the $C D$ and N-terminal tail of SWI6 causing disruption of SWI6-H3K9me3 interaction (62). We propose that biochemical purification of HPL-2 to homogeneity could lead to elimination of allosteric regulation of the protein restoring its ability to interact with $\mathrm{H} 3 \mathrm{~K} 9 \mathrm{me} 3$. 


\subsubsection{Possible PTMs of HPL-2 CD might negatively affect its affinity to H3K9me3}

Another possibility worth considering is PTM of HPL-2 blocks its interaction with H3K9me3 in vivo. Phosphorylation of HP1 proteins is known to have either positive or negative effects on their interaction with H3K9me3 (130). HPL-2 phosphorylation in vivo has recently been reported (84). Phosphomimic mutants of HPL-2 are able to better repress transcription of odr-1 locus (84). This suggested that phosphomimic mutants of HPL-2 are able to work in a manner similar to HPL-2 phosphorylation. Therefore, we made these recombinant phosphomimic mutants (Ser to Glu) to test whether phosphorylation interferes with the ability of HPL-2 to interact with H3K9me3. We did not find any effect of any of these phosphomimics on HPL-2-H3K9me3 interaction. It remains to be seen whether HPL-2 CD undergoes any other PTMs such as methylation, acetylation in vivo that disrupts its interaction with H3K9me3. PTM screen of HPL-2 and their effect on HPL-2-H3K9me3 interaction may unravel this novel mode of regulation in C. elegans that could possibly be conserved in mammals. Another approach would be direct comparison of HPL-2 with its homologue HPL-1 (since HPL-1 is not LIN-61 dependent and is highly similar to HPL-2) and scanning mutagenesis of unique regions found in HPL-2 to identify the regions in HPL-2, that are subjected to this regulation.

Our observation that HPL-2 does not directly interact with $\mathrm{H} 3 \mathrm{~K} 9 \mathrm{me} 3$ in vivo raises several questions in the field of HP1 biology. To our knowledge, there is no evidence of HP1-H3K9me3 physical interaction in vivo. All studies so far have relied on immunofluorescence localization of HP1 to pericentromeric heterochromatin and its colocalization with $\mathrm{H} 3 \mathrm{~K} 9 \mathrm{me} 3$ or ChIP assays that determines co-occupancy. Both these experiments are indicative of co-occurrence of two factors, but they do not necessarily mean that the two factors physically associate with each other. CD mutants of HP1 proteins delocalize from pericentromeric heterochromatin. While this suggests the involvement of $C D$ in their heterochromatic localization, this does not mean that $C D$ directly interacts with H3K9me3 in vivo. Moreover, it has been shown that HP1 localization to chromatin relies on several auxiliary factors (57). Thus, although HP1 proteins have the ability to bind $\mathrm{H} 3 \mathrm{~K} 9 \mathrm{me} 3$ in vitro, this interaction appears to be highly regulated and may involve additional layers of complexity, beyond the binding to the histone modification. 


\subsection{LIN-61 is a direct interaction partner of HPL-2}

We show that HPL-2 directly interacts with LIN-61 via its CSD in vitro. Also, these factors immunoprecipitate each other from $C$. elegans nuclear extract. HP1 proteins are known to mediate protein-protein interactions via their CSD (134). These interactions are largely attributed to PXVXL motif present in the target proteins that makes contact with CSD $(134,135)$. Nuclear proteins such as the Lamin B receptor, CAFp150 interact with HP1 proteins in PXVXL dependent manner (136). We did not find any PXVXL or a related motif in the LIN-61 protein. Nevertheless, presence of a PXVXL motif is not an absolute requirement for CSD-mediated protein-protein interactions. It has been shown that HP1 proteins interact with several proteins in a PXVXL independent manner. This includes BRG1 and SUV39H $(137,138)$. In fact, HP1Y interacts with Ku-70 via its SLSDSES motif (133). We conclude that LIN-61 might belong to a growing list of factors that interact with HP1 proteins in PXVXL independent manner.

\subsubsection{Biological role of LIN-61 mediated HPL-2-H3K9me3 interaction}

hpl-2 has been implicated in vulva and germline development (104). Originally, it has been classified as SynMuv B gene (104). However, it shows genetic interaction with several genes that have been classified as SynMuv B regulators (97). This suggests that $h p l-2$ acts in both SynMuv A and SynMuv B pathways. lin-61 was identified as a SynMuv B gene in a genetic screen for vulval mutants in a SynMuv A mutant background (99). Earlier study from our group has shown that LIN-61 and its interaction with H3K9me3 is essential for vulva development as a SynMuv B regulator (97). The study also reported a genetic interaction of lin-61 (tm2649) with hpl-2 (tm1489).

These reports are difficult to reconcile in the context of our biochemical findings on these two factors. lin-61 and hpl-2 genes genetically interact during vulva development while LIN-61 and HPL-2 proteins biochemically interact with each other. We propose that both LIN-61 and HPL-2 regulate vulva development via multiple biochemical pathways.

Our analysis of lin-61 (n3447) allele, a loss of function mutant for H3K9me3-LIN-61HPL-2 suggests that this biochemical pathway is crucial for vulva development. $C$. elegans homologue of SUV39H H3K9 methyltransferase, met-2 (tm4256) has also been 
classified as SynMuv B regulator (129). This suggests that $\mathrm{H} 3 \mathrm{~K} 9$ methylation is required for vulva development. Interestingly, our analysis of met-2 (tm4256) with hpl-2 and lin61 shows that it genetically interacts with both of them. This suggests that HPL-2 and LIN-61 regulates vulva development both in H3K9me dependent as well as H3K9me independent manner. H3K9me independent recruitment of HPL-2 and LIN-61 might involve transcription factor mediated recruitment of these proteins to chromatin. HPL-2 is known to associate with LIN-13 (117). It has also been proposed that HPL-2 and LIN35 coordinately bind chromatin at many sites (220). We conclude that H3K9me3-HPL-2LIN-61 biochemical pathway is required for vulva development in $C$. elegans. Interestingly, HPL-2 and LIN-61 also regulate vulva development independent of H3K9 methylation.

\subsection{LIN-13, a zinc finger protein also binds HPL-2}

\subsubsection{HPL-2-LIN-61-LIN-13 complex}

We found that LIN-13 also recovers on H3K9me3 peptides in LIN-61 as well as HPL-2 dependent manner. We also found that all three factors can immunoprecipitate each other. We conclude that these three factors form HPL-2-LIN-61-LIN-13 complex. Although, HPL-2 interacts with both LIN-61 and LIN-13 via its CSD, full length HPL-2 is required for HPL-2-LIN-61-LIN-13 complex formation. We hypothesize that HPL-2 multimerization might be required for this complex formation. Upon multimerization, multiple CSD molecules can interact simultaneously with LIN-61 and LIN-13 forming HPL-2-LIN-61-LIN-13 complex. HPL-2 mutants that are defective in dimerization but not in its interaction with LIN-61 and LIN-13 are required to further explain the complex formation. In agreement to this, we found that HPL-2 has the ability to form multimers in vivo. However, the mechanism of HPL-2 multimerization is still unknown. HP1 proteins are known to multimerize via their CSD (194). A recent report in yeast on SWI6 suggests that HP1 proteins can also multimerize via their CD and promote heterochromatin spreading (33). 


\subsubsection{HPL-2 also forms distinct complexes with LIN-61 and LNI-13}

Our results also show that LIN-13 and HPL-2 interact with each other even in the absence of LIN-61 although the interaction is reduced. Besides, interaction between LIN-61 and LIN-13 is lost in the absence of HPL-2. This raises three possibilities about of how these factors interact with each other to form multiple complexes (figure 4.2).

1. HPL-2/LIN-61/LIN-13 form a complex that relies on full length HPL-2 and its multimerization (figure 4.2A).

2. LIN-61 and HPL-2 interact with each other independently of LIN-13 (figure4.2B).

3. LIN-13 interacts with HPL-2 independently of LIN-61 (figure 4.2C).

(A)

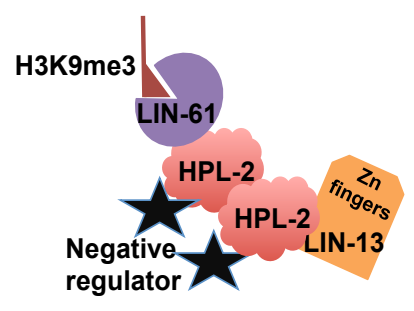

(B)

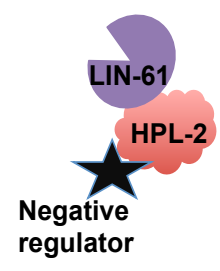

(C)

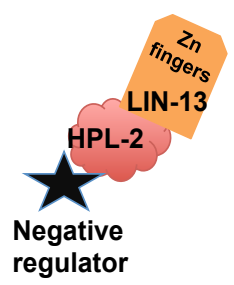

Figure 4.2: HPL-2/LIN-61/LIN-13 can form multiple complexes

(A) LIN-61 binds histone modification via its MBT domains while HPL-2 via its CSD binds LIN-61. HPL-2 multimerization leads to LIN-13 binding to HPL-2-CSD leading to formation of HPL-2/LIN-61/LIN-13 complex. (B) LIN-61 and HPL-2 interact with each other to form HPL-2-LIN-61 complex. (C) LIN-13 and HPL-2 interact with each other to form HPL-2-LIN-13 complex. 
These complexes can be recruited to chromatin in $\mathrm{H} 3 \mathrm{~K} 9 \mathrm{me} 3$ dependent as well as H3K9me3 independent manner.

We conclude that HPL-2, LIN-61 and LIN-13 form a multimeric complex as well as distinct HPL-2-LIN-13 and HPL-2-LIN-61 complexes. This is also consistent with previous studies, which report that HPL-2-RFP foci partially co-localize with LIN-13-GFP foci (105) while HPL-2-GFP foci partially co-localize with LIN-61-FLAG foci (117).

\subsection{LIN-13, acts in both SynMuv A as well as SynMuv B pathway of vulva development}

We show that loss of LIN-13 using lin-13 RNAi leads to highly penetrant muv phenotype and sterility in wild type N2 worms. Moreover, the phenotype caused by loss of lin-13 is temperature sensitive. It has been proposed that lin-13 acts as SynMuv B gene at $15^{\circ} \mathrm{C}$ where as at $20^{\circ} \mathrm{C}$, loss of lin-13 alone leads to muv phenotype. Among HPL-2, LIN-61 and LIN-13, lin-13 has the most severe phenotype. However, our analysis suggests that loss of lin-13 also causes stark reduction in HPL-2 protein levels as well as slight reduction in LIN-61 protein levels without affecting their mRNA levels. Therefore, the severe phenotype observed upon loss of lin-13 cannot be attributed to LIN-13 functions alone. We propose that loss of LIN-13 compromises the stability of HPL-2/LIN-61/LIN13 complexes and hence make these factors prone to degradation. Interestingly, both HPL-2 and LIN-61 negatively regulate the expression of lin-13 at transcriptional level. This points to the existence of complex negative feed back regulation among HPL-2, LIN-61 and LIN-13.

We further show that loss of LIN-13 does not affect LIN-61-H3K9me3 interaction. However, because loss of LIN-13 reduces HPL-2 protein levels, we could not determine the effects of loss of LIN-13 on HPL-2-LIN-61 interaction. An earlier study has shown that lin-13 RNAi in hpl-2::gfp worms causes loss of GFP foci in embryos in immunofluorescence assays. The study proposed that $\mathrm{LIN}-13$ regulates recruitment of HPL-2 to chromatin (117). However, in our RNAi assays, we found a stark reduction in protein levels of HPL-2 upon lin-13 RNAi. Thus, we argue that loss of GFP foci in immunofluorescence assays is likely due to a reduction in GFP-tagged HPL-2 protein levels. 
We show that lin-13 genetically interacts with $h p l-2$ and lin-61 during vulva development. This suggests that residual HPL-2-LIN-61 protein complex in lin-13 RNAi worms also regulates vulva development independent of LIN-13. These results also support the existence of multiple complexes among LIN-13, HPL-2 and LIN-13. We propose that these complexes regulate transcription of distinct subsets of target genes important for vulva development in C. elegans.

\subsection{HPL-2/LIN-61/LIN-13 regulate transcription of genes associated with vulva development and fertility in C. elegans}

We compared the gene expression profiles of hpl-2, lin-61 mutants and lin-13 RNAi worms with wild type N2 worms at L3 stage. We identified several genes that are commonly regulated by these three factors. GO analysis of this subset of genes revealed high enrichment for the genes associated with vulva development and reproduction. These include prg-1, pgl-1, puf-1, which are known regulators of vulva development $(86,87)$. Our results shed light on transcription regulation by HPL-2, LIN61 and LIN-13 and its link to developmental pathways in C. elegans. HP1 proteins in flies and mammals are known to regulate various developmental processes (123). HP1 $\beta$ is required for development of cerebral cortex and neuromuscular junctions in mice (51). Loss of HP1a impairs early development in flies (123).

We found that some of the genes identified in our transcriptome analysis are bound by HPL-2, LIN-61 and LIN-13 in ChIP-seq analysis representing the direct targets of these factors. Interestingly, lin-13 was identified as one of the targets of HPL-2 and LIN-61, which is upregulated in $h p l-2$ and lin-61 mutants, suggesting the feed back regulation by HPL-2/LIN-61/LIN-13 complex. We propose that HPL-2, LIN-61 and LIN-13 during L3 stage regulate expression of key genes in vulval precursor cells as well as anchor cell to allow precise lin-3 mediated vulval induction.

Loss of these factors causes ectopic upregulation of several genes that leads to excessive lin-3 signaling resulting in muv phenotype. Consistently, we found that lin61; hpl-2 mutant and lin-13 RNAi worms have 2-fold increased expression of lin-3 at L3 stage, that leads to excessive lin-3 signaling and results in muv phenotype. Earlier study has suggested that 2-fold increased expression of lin-3 is sufficient to drive muv phenotype in C. elegans (129). One possible mechanism by which lin-13 RNAi worms 
develop muv phenotype is by mimicking the lin-61; $h p l-2$ mutant as the levels of both these proteins are reduced upon loss of lin-13.

It has been proposed that LIN-61, HPL-2 and LIN-13 containing complex represses germline RNAi pathways in somatic tissues (105). We observed over-representation of genes involved in ncRNA processing and metabolism. We identified that HPL-2-LIN-61LIN-13 complex is bound at the promoters of 21U-RNA genes. These mutants might exhibit ectopic expression of 21U-RNA species and germline specific RNAs in somatic tissues and are therefore required for repression of germline specific ncRNA genes in somatic tissues.

\subsection{H3K9me3 and HPL-2 distribution does not correlate in vivo}

We overlapped the binding sites of HPL-2 and H3K9me3 obtained from ChIP-seq analysis. We found that there exists little overlap (10\%) suggesting that HPL-2 recruitment to chromatin occurs primarily in H3K9me3 independent manner. An earlier study has reported that broad distribution of HPL-2 in gene bodies is diminished in intensity in the absence of H3K9 methylation although its recruitment is not affected (83). Studies on HP1 proteins in mammals and flies suggest that HP1 localization partially depends on H3K9me3 (123). H3K9me3 independent mechanism(s) of recruitment of HP1 proteins are known (50). ncRNAs have also been implicated in the recruitment of HP1 proteins (66). We propose that HPL-2 recruitment primarily occurs either via its interaction with protein factors such as LIN-13, LIN-61 or via its interaction with RNA. 


\subsection{Genome-wide distribution studies of HPL-2, LIN-61 and LIN-13 confirm the existence of multiple complexes among HPL-2, LIN-13 and $L I N-61$}

We analyzed the modENCODE ChIP-seq datasets of HPL-2, LIN-61 and LIN-13. When, we overlapped the binding sites of HPL-2, LIN-61 and LIN-13, we found that there exist 3 subsets of binding sites corresponding to HPL-2-LIN-61-LIN-13, HPL-2LIN61 and HPL-2-LIN-13. These results are consistent with our biochemical findings.

\subsubsection{HPL-2-LIN-13 complex}

We found that HPL-2-LIN-13 binding sites are sharp and occupy mainly promoters. Sharp binding sites mainly reflect the transcription factor binding, which recognize a sequence motif in promoter regions (216).

We propose that LIN-13 acts as transcription factor and recognize specific sequence motif in the DNA in promoter regions and via its interaction with HPL-2, recruits HPL-2 to chromatin (figure 4.3). It has been found that LIN-13 localizes to nucleus and forms nuclear speckles (142). These nuclear speckles are similar to the ones observed in a study showing binding of ELT-2-GFP to ELT-2 motif (142).

In mammals, HP1 proteins are known to interact with $\mathrm{Rb}$ and bind specific promoters to repress transcription (54). Similarly, C. elegans HPL-2 also binds to LIN-13 and performs similar function(s).

\subsubsection{HPL-2-LIN-61 complex}

We found that HPL-2-LIN-61 binding sites have broad distribution. Broad distribution reflects binding to a histone modification or ncRNA (217). Some histone modifications such as H3K27me3 that are associated with heterochromatin have broad distribution patterns, whereas other modifications such as H3K4me1, H3K4me2 that are associated with enhancer function have sharp distribution pattern (217). In C. elegans, RNAi mediated chromatin silencing also leads to formation of broad H3K9me3 domains over target genes (82). The small RNA directs the recruitment of H3K9me3 enzymatic machinery to target genes (82).

We propose that HPL-2-LIN-61 recruitment is either based on histone modification or 
ncRNA (figure 4.3). The broadness of the binding sites also suggests that this complex has the ability to spread along the chromatin. Multimerization of HPL-2 and LIN-61 might contribute to the phenomena. Although, the complex binds H3K9me3 and could be recruited to chromatin via $\mathrm{H} 3 \mathrm{~K} 9 \mathrm{me} 3$, our ChIP-seq analysis suggested that there exists little overlap of this complex with H3K9me3. We therefore, propose that HPL-2LIN-61 complex is recruited by intronic RNA species, which then spreads on both sides covering the entire gene occasionally. Intronic RNAs are known to mediate recruitment of PRC2 via their interaction with EZH2 (196).

\subsection{RNase sensitivity of HPL-2/LIN-61/LIN-13 complexes suggests the involvement of RNA component in the stability of these complexes}

Our analysis of HPL-2/LIN-61/LIN-13 complexes using RNase A sensitivity assays suggests that an RNA component is involved in the stability of these complexes in $C$. elegans. We show that RNase A treatment in peptide pull down assays abolishes HPL2 but not LIN-61 binding to H3K9me3 peptides. Similarly, LIN-13 binding to HPL-2 is reduced in immunoprecipitation assays upon $\mathrm{RNase} A$ treatment.

Since, HPL-2 directly interacts with both LIN-61 and LIN-13, we propose that RNA association may stabilize these interactions further. Moreover, RNA association might be crucial for recruitment of HPL-2 associated complexes to chromatin. RNA mediated recruitment of HP1 family proteins in mammalian systems has been recently uncovered (1.5.2.3). HPL-2 has recently been implicated in nuclear RNAi pathways (215) suggesting that RNAi and heterochromatin formation and maintenance might be interlinked in C. elegans. 


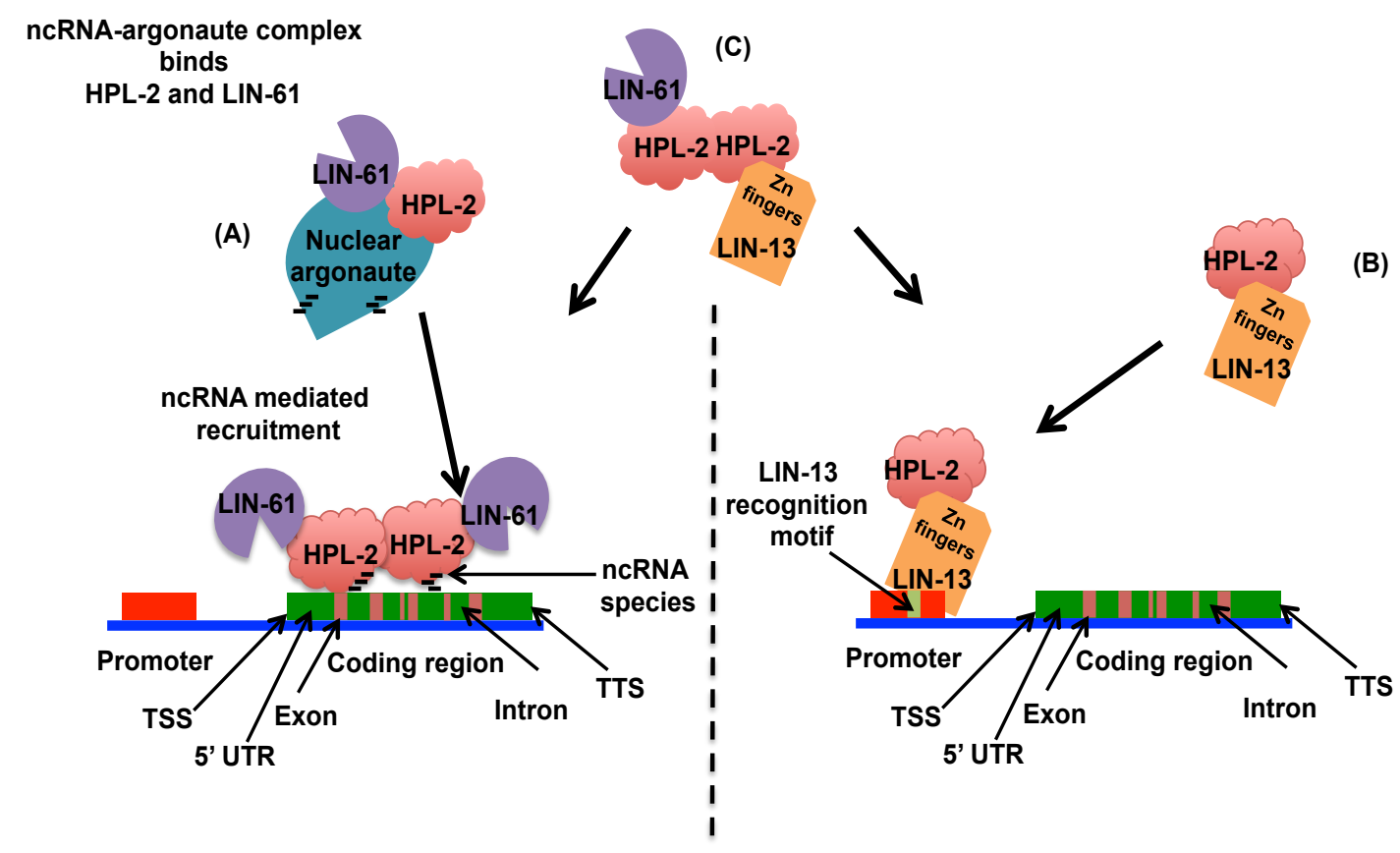

Figure 4.3: Proposed model for recruitment of HPL-2/LIN-61/LIN-13 complexes

(A) Nuclear argonaute binds small RNAs. This leads to binding of HPL-2 and LIN-61 to argonaute-RNA complex. Based on the homology of the small RNA, the complex gets recruited to homologous intronic regions. Once bound, HPL-2 and LIN-61 can spread on both sides upto few $\mathrm{kb}$. (B) LIN-13 binds specifically to specific recognition motif in the DNA, thus recruiting LIN-13-HPL-2 complex to specific promoter regions in $C$. elegans. (C) HPL-2-LIN-61-LIN-13 complex can be recruited to chromatin via both the mechanisms. ncRNA mediated recruitment leads to enrichment of HPL-2-LIN-61-LIN-13 complex to intronic regions in genes. LIN-13 recognition motif mediated recruitment leads to enrichment of HPL-2-LIN-61-LIN-13 complex to promoter regions. 


\subsection{Conclusion}

The work presented in this thesis identified putative HP1 complexes in C. elegans. We defined putative recruitment mechanisms of different HP1 associated complexes. Our results provide novel insights in transcription regulation by these complexes during vulva development in C. elegans.

Our findings suggest that the $C$. elegans HP1 homologue; HPL-2 does not directly bind H3K9me3, unlike what has been proposed for HP1 family proteins. Interestingly, HPL-2 depends on LIN-61 for association with H3K9me3. Our analysis of LIN-61-H3K9me3 interaction defective mutant suggests that HPL-2-LIN-61-H3K9me3 interaction is required for vulva development in $C$. elegans.

We further demonstrate that HPL-2, LIN-61 and LIN-13 biochemically interact with each other to form multiple complexes. We show that HPL-2 interacts with both LIN-61 and LIN-13 via its CSD. Moreover, we show that LIN-61 and LIN-13 do not directly interact with each other in the absence of HPL-2. Consequently, full length HPL-2 and possibly its multimerization is required for LIN-61-HPL-2-LIN-13 complex formation.

Our results suggest the existence of at least three putative complexes among HPL-2, LIN-13 and LIN-61. These complexes bind distinct, non-overlapping subsets of target genes and regulate their transcription including genes associated with vulva development. In agreement, $h p /-2$, lin-61 and lin-13 genetically interact with each other during vulva development.

Analysis of the binding sites of HPL-2-LIN-61 complex and HPL-2-LIN-13 complex reveals two distinct modes of chromatin association of HPL-2. Sharp distribution of HPL-2-LIN-13 complex points to the transcription factor mediated recruitment. Broad distribution of HPL-2-LIN-61 complex points to ncRNA mediated recruitment. Our results on involvement of a RNA component in the stability of HPL-2-LIN-61-LIN13 complexes reiterate our findings.

Overall, our studies highlight the role of HP1 associated complexes in transcription regulation in vulva development in $C$. elegans. 


\subsection{Future perspectives}

Our results on LIN-61 mediated HPL-2-H3K9me3 interaction challenge the current proposed model on HP1-H3K9me3 interaction in mammals. Further insights into the proposed LIN-61 mediated recruitment of HPL-2 to chromatin will unravel novel mode of HPL-2-chromatin association. ChIP analysis of HPL-2 in lin- 61 mutant will lead to the identification of actual chromatin binding sites of HPL-2 that depend solely on LIN-61. However, the negative regulation of HPL-2-H3K9me3 association in vivo needs to be addressed. Molecular scanning mutagenesis of HPL-2 promises to lead the identification of this negative regulation. It is likely that this negative regulation of $C D$ functionality has implications in vulva development and fertility in C. elegans. In particular, identification and biochemical analysis of loss of function mutants of HPL-2LIN-61 interaction will provide further insights into its biological significance.

It will also be important to understand whether LIN-13 binds to chromatin solely in a transcription factor dependent manner or whether it also relies on HPL-2 for recruitment to a subset of its targets. ChIP analysis of LIN-13 in hpl-2 mutants will help understand the role of HPL-2 in LIN-13 binding to chromatin. Identification of LIN-13 consensus motif based on its ChIP-seq binding sites will provide insights into its recruitment mechanism to chromatin.

C. elegans has played a major role in the identification and dissection of regulatory mechanisms of endogenous RNAi pathways. Several classes of RNA molecules have been identified that regulate gene expression. RNA-protein interactions are increasingly becoming evident in the regulation of gene expression. Our results on possible involvement of RNA in the formation of HPL-2/LIN-61/LIN-13 complexes demand the identification of these RNA species. PAR-CLIP experiments could lead the way forward in the identification of these RNA species associated with HPL-2, LIN-13 or LIN-61. Using genetics tools that are available in C. elegans, elucidating the biological significance of these interactions will be of high interest. It is beyond doubt that understanding the molecular mechanisms of HPL-2 function in C. elegans will help uncover additional layers of complexities that underlie HP1 functions in mammals. 


\section{$5 \quad$ References}

1. Luger, K., Mader, A. W., Richmond, R. K., Sargent, D. F., Richmond, T. J. Crystal structure of the nucleosome core particle at $2.8 \mathrm{~A}$ resolution. Nature. 1997. 389: 251.

2. Tsankova, N., Renthal, W., Kumar, A., Nestler, E. J. Epigenetic regulation in psychiatric disorders. Nat. Rev. Neurosci. 2007. 8:356.

3. Jenuwein, T., Allis C. D. Translating the histone code. Science. 2001. 293(5532): 1074-80.

4. Campos, E. I., Reinberg, D. Histones: annotating chromatin Annu. Rev. Genet. 2009.43: 559-599.

5. Ernst, J., et al. Mapping and analysis of chromatin state dynamics in nine human cell types. Nature. 2011. 473: 43-49.

6. Zhou, V. W., Goren, A., Bernstein, B. E. Charting histone modifications and the functional organization of mammalian genomes. Nature Rev. Genet. 2011. 12: 7-18.

7. Filion G. J., van, Bemmel, J. G., Braunschweig, U., et al. Systematic protein location mapping reveals five principal chromatin types in Drosophila cells. Cell 2010; 143: 212-224.

8. Fischle, W., Wang, Y., Allis, C. D. Binary switches and modification cassettes in histone biology and beyond. Nature. 2003. 425:475-479.

9. Kouzarides, T. Chromatin modifications and their function. Cell. 2007. 128: 693-705. 
10. Selvi, B. R., Mohankrishna, D. V., Ostwal, Y. B., Kundu, T. K. Small molecule modulators of histone acetylation and methylation: a disease perspective. Biochim Biophys Acta. 2010. 1799(10-12): 810-28.

11. Turner, B. M. Histone acetylation and an epigenetic code. Bioessays 2000. 22: 836.

12. Cheung, P., Allis, C. D., Sassone-Corsi, P. Signaling to chromatin through histone modifications. Cell. 2000. 103: 263.

13. Strahl, B. D., Allis, C. D. The language of covalent histone modifications. Nature. 2000. 403: 41-45.

14. Lee, J. S., Smith, E., Shilatifard, A. The language of histone crosstalk. Cell. 2010. 142: 682-685.

15. Yun, M., Wu, J., Workman, J. L., Li, B. Readers of histone modifications. Cell Res. 2011. 21(4): 564-78.

16. Musselman, C. A., Lalonde, M. E., Côté, J., Kutateladze, T. G. Perceiving the epigenetic landscape through histone readers. Nat. Struct. Mol. Biol. 2012. 19(12): 1218-27.

17. Daujat, S., Bauer, U. M., Shah, V., Turner, B., Berger, S., Kouzarides, T. Crosstalk between CARM1 methylation and CBP acetylation on histone H3. Curr. Biol. 2002.12(24): 2090-7.

18. Xu, W., Cho, H., Evans, R. M. Acetylation and methylation in nuclear receptor gene activation. Methods. Enzymol. 2003. 364:205-23.

19. Metzger, E., Imhof, A., Patel, D., Kahl, P., Hoffmeyer, K., Friedrichs, N., Müller, J. M., Greschik, H., Kirfel, J., Ji, S., Kunowska, N., Beisenherz-Huss, C., Günther, T., Buettner, R., Schüle, R. Phosphorylation of histone H3T6 by PKCbeta(I) controls demethylation at histone H3K4. Nature. 2010. 464(7289): 792-6. 
20. Rountree, M. R., Bachman, K. E., Baylin, S. B. DNMT1 binds HDAC2 and a new co-repressor, DMAP1, to form a complex at replication foci. Nat. Genet. 2000 . 25(3):269-77.

21. Li, P., Wang, D., Yao, H., Doret, P., Hao, G., Shen, Q., Qiu, H., Zhang, X., Wang, Y., Chen, G., Wang, Y. Coordination of PAD4 and HDAC2 in the regulation of p53-target gene expression. Oncogene. 2010. 29(21):3153-62.

22. Schmitges, F. W., Prusty, A. B, Faty, M., Stützer, A., Lingaraju, G. M., Aiwazian, J., Sack, R., Hess, D., Li, L., Zhou, S., Bunker, R. D., Wirth, U., Bouwmeester, T., et al. Histone methylation by PRC2 is inhibited by active chromatin marks. Mol Cell. 2011. 42(3): 330-41.

23. Pray-Grant, M. G., Daniel, J. A., Schieltz, D., Yates, J. R., Grant P. A. Chd1 chromodomain links histone H3 methylation with SAGA- and SLIK-dependent acetylation. Nature. 2005. 433(7024): 434-8.

24. Li, B., Carey, M., Workman, J. L. The role of chromatin during transcription. Cell. 2007. 128: 707-719.

25. Berger, S.L. The complex language of chromatin regulation during transcription. Nature. 2007. 447: 407-41.

26. James, T. C., Elgin, S. C. Identification of a non-histone chromosomal protein associated with heterochromatin in Drosophila melanogaster and its gene. Mol. Cell. Biol. 1986. 6:3862-72.

27. Eissenberg, J. C., James, T. C., Foster-Hartnett, D. M., Hartnett, T., Ngan, V., Elgin, S. C. Mutation in a heterochromatin-specific chromosomal protein is associated with suppression of position effect variegation in Drosophila melanogaster. Proc. Natl. Acad. Sci. 1990. 87:9923-27.

28. Zeng, W., Ball, A. R. Jr., Yokomori, K. HP1: heterochromatin binding proteins working the genome. Epigenetics 2010. 5:287-292. 
29. Bannister, A. J., Zegerman, P., Partridge, J. F., Miska, E. A., Thomas, J. O., Allshire, R. C., and Kouzarides, T. Selective recognition of methylated lysine 9 on histone H3 by the HP1 chromo domain. Nature, 2001. 410: 120-124.

30. Nielsen, P. R., Nietlispach, D., Mott, H. R., Callaghan, J., Bannister, A, Kouzarides, T., Murzin, A. G., Murzina, M. V., Laue, E. D. Structure of the HP1 chromodomain bound to histone $\mathrm{H} 3$ methylated at lysine 9 Nature, 2002, 416: 103-107.

31. Kaustov, L., Ouyang, h., Amaya, M., Lemak, A., Nady, N., Duan S., Wasney G. A., Li Z., Vedadi M., Schapira M., et al. Recognition and specificity determinants of the human cbx chromodomains J. Biol. Chem. 2011, 286:521529.

32. Munari, F., Soeroes, S., Zenn, H. M., Schomburg, A., Kost, N., Schröder, S., Klingberg R., Rezaei-Ghaleh, N., Stützer A., Gelato K. A., et al. Methylation of lysine 9 in histone $\mathrm{H} 3$ directs alternative modes of highly dynamic interaction of heterochromatin protein hHP1 $\beta$ with the nucleosome J. Biol. Chem. 2012. 287: 33756-33765.

33. Canzio, D., Chang, E. Y., Shankar, S., Kuchenbecker, K. M., Simon, M.D., Madhani, H. D., Narlikar, G. J., Al-Sady B. Chromodomain-mediated oligomerization of HP1 suggests a nucleosome-bridging mechanism for heterochromatin assembly Mol. Cell, 41: 67-81.

34. Canzio, D., Liao, M., Naber, N., Pate, E., Larson, A., Wu, S., Marina, D. B., Garcia, J. F., Madhani, H. D., Cooke, R., Schuck, P., Cheng, Y., Narlikar, G. J. A conformational switch in HP1 releases auto-inhibition to drive heterochromatin assembly. Nature. 2013.496(7445): 377-81.

35. Yap, K. L., Zhou, M. M. Structure and mechanisms of lysine methylation recognition by the chromodomain in gene transcription. Biochemistry, 2011. 50: 1966-1980.

36. Daujat, S., Zeissler, U., Waldmann, T., Happel, N., Schneider, R., HP1 binds specifically to Lys26-methylated histone $\mathrm{H} 1.4$, whereas simultaneous Ser27 phosphorylation blocks HP1 binding. J. Biol. Chem., 2005. 280: 38090-38095. 
37. Chin, H.G., Esteve, P.O., Pradhan, M., Benner, J., and Patnaik, D., et al. Automethylation of G9a and its implication in wider substrate specificity and HP1 binding. Nucleic Acids Res., 2007. 35: 7313-7323.

38. Ruan, J., Ouyang, H., Amaya, M.F., Ravichandran, M., Loppnau, P., Min, J., Zang, J., Structural basis of the chromodomain of $\mathrm{Cbx} 3$ Bound to methylated peptides from histone $\mathrm{H} 1$ and G9a. PLoS ONE, 2012.7: e35376.

39. James, T.C., Eissenberg, J.C., Craig, C., Dietrich, V., Hobson, A., Elgin, S.C., Distribution patterns of HP1, a heterochromatin-associated nonhistone chromosomal protein of Drosophila. Eur. J. Cell. Biol., 1989. 50: 170-180.

40. Greil, F., Van, der, Kraan, I., Delrow, J., Smothers, J.F., de, Wit, E., Bussemaker, H.J., Van, Driel, R., Henikoff, S., Van, Steensel, B., Distinct HP1 and Su(var)3-9 complexes bind to sets of developmentally coexpressed genes depending on chromosomal location. Genes. Dev., 2003. 17: 2825-2838.

41. Fanti, L., Berloco, M., Piacentini, L., Pimpinelli, S., Chromosomal distribution of heterochromatin protein 1 (HP1) in Drosophila: a cytological map of euchromatic HP1 binding sites. Genetica, 2003. 117: 135-147.

42. Peters, A. H., O'Carroll, D., Scherthan, H., Mechtler, K., Sauer, S., Schoefer, C., Weipoltshammer, K., Pagani, M., Lachner, M., Kohlmaier, A., Opravil, S., Doyle, M., Sibilia, M., and Jenuwein, T. Loss of the Suv39h histone methyltransferases impairs mammalian heterochromatin and genome stability. Cell, 2001. 107: 323-337.

43. Peters, A. H., Mermoud, J. E., O'Carroll, D., Pagani, M., Schweizer, D., Brockdorff, N., Jenuwein, T. Histone H3 lysine 9 methylation is an epigenetic imprint of facultative heterochromatin. Nat. Genet. 2002. 30:77-80.

44. Towbin, B. D., González-Aguilera, C., Sack, R., Gaidatzis, D., Kalck, V., Meister, P., Askjaer, P., Gasser, S. M. Step-wise methylation of histone H3K9 positions heterochromatin at the nuclear periphery. Cell, 2012. 150: 934-947. 
45. Pinheiro, I., Margueron, R., Shukeir, N., Eisold, M., Fritzsch, C., Richter, F.M., Mittler, G., Genoud, C., Goyama, S., Kurokawa, M., et al. Prdm3 and Prdm16 are $\mathrm{H} 3 \mathrm{~K} 9 \mathrm{me} 1$ methyltransferases required for mammalian heterochromatin integrity. Cell, 2012. 150: pp. 948-960.

46. Eissenberg, J. C., Morris, G. D., Reuter, G., Hartnett, T. The heterochromatinassociated protein HP-1 is an essential protein in Drosophila with dosage dependent effects on position-effect variegation. Genetics, 1992. 131: 345352.

47. Kellum, R., Alberts, B. M. Heterochromatin protein 1 is required for correct chromosome segregation in Drosophila embryos. J. Cell Sci., 1995. 108: 1419-1431.

48. Tschiersch, B., Hofmann, A., Krauss, V., Dorn, R., Korge, G., Reuter, G. The protein encoded by the Drosophila position effect variegation suppressor gene Su(var)3-9 combines domains of antagonistic regulators of homeotic gene complexes. EMBO J, 1994. 13: 3822-3831.

49. Schotta, G., Ebert, A., Krauss, V., Fischer, A., Hoffmann, J., et al. Central role of Drosophila SU(VAR)3-9 in histone H3-K9 methylation and heterochromatic gene silencing. EMBO J, 2002. 21: 1121-1131.

50. Figueiredo, M.L.A., Philip, P., Stenberg, P., Larsson, J. HP1a recruitment to promoters is independent of H3K9 methylation in Drosophila melanogaster. PLoS Genet, 2012. 8: e1003061.

51. Aucott, R., Bullwinkel, J., Yu, Y., Shi, W., Billur, M., Brown, J.P., Menzel, U., Kioussis, D., Wang, G., Reisert, I., Weimer, J., Pandita, R.K., Sharma, G.G., Pandita, T.K., Fundele, R., Singh, P.B. HP1- $\beta$ is required for development of the cerebral neocortex and neuromuscular junctions. J. Cell Biol., 2008. 183: 597-606.

52. Richart, A. N., Brunner, C. I. W., Stott, K., Murzina, N. V., Thomas, J. O. Characterization of chromoshadow domain-mediated binding of heterochromatin protein 1a (HP1 $\alpha)$ to histone H3. J. Biol. Chem., 2012. 287: 18730-18737 
53. Smallwood, A., Black, J. C., Tanese, N., Pradhan, S., Carey, M. HP1-mediated silencing targets Pol II coactivator complexes. Nat. Struct. Mol. Biol. 2008. 15: 318-20.

54. Nielsen, S. J., Schneider, R., Bauer, U. M., Bannister, A. J., Morrison, A., O'Carroll D., Firestein, R., Cleary, M., Jenuwein, T., Herrera, R. E., Kouzarides, $\mathrm{T}$. Rb targets histone H3 methylation and HP1 to promoters. Nature. 2001. 412: $561-565$.

55. Fischle, W., Wang, Y., Jacobs, S. A., Kim, Y., Allis, C. D., Khorasanizadeh, S. Molecular basis for the discrimination of repressive methyl-lysine marks in histone $\mathrm{H} 3$ by Polycomb and HP1 chromodomains. Genes Dev., 2003.17:1870-1881.

56. Fischle, W., Tseng, B. S., Dormann, H. L., Ueberheide, B. M., Garcia, B. A., Shabanowitz, J., Hunt, D. F., Funabiki, H., Allis, C. D. Regulation of HP1chromatin binding by histone $\mathrm{H} 3$ methylation and phosphorylation. Nature, 2005. 438: 1116-1122.

57. Eskeland, R., Eberharter, A., Imhof, A. HP1 binding to chromatin methylated at H3K9 is enhanced by auxiliary factors. Mol. Cell. Biol., 2007. 27: 453-465.

58. Hiragami, H. K., and Fischle, W. RNAs - physical and functional modulators of chromatin reader proteins. Biochim. Biophys. Acta. 2014. 1839(8): 737-42. Review.

59. Akhtar, A., Zink, D., and Becker, P.B., Chromodomains are protein-RNA interaction modules. Nature, 2000. 407: 405-409.

60. Bernstein, E., Duncan, E. M., Masui, O., Gil, J., Heard, E., and Allis, C. D. Mouse polycomb proteins bind differentially to methylated histone $\mathrm{H} 3$ and RNA and are enriched in facultative heterochromatin. Mol. Cell. Biol., 2006. 26: 2560-2569. 
61. Muchardt, C., Guilleme, M., Seeler, J. S., Trouche, D., Dejean, A., Yaniv, M. Coordinated methyl and RNA binding is required for heterochromatin localization of mammalian HP1alpha. EMBO rep., 2002. 3: 975-981.

62. Keller, C., Adaixo, R., Stunnenberg, R., Woolcock, K.J., Hiller, S., Buhler, M., HP1(Swi6) mediates the recognition and destruction of heterochromatic RNA transcripts. Mol. cell, 2012. 47: 215-227.

63. Maison, C., Bailly, D., Roche, D., Montes, de, Oca, R., Probst, A. V., Vassias, I., Dingli, F., Lombard, B., Loew, D., Quivy, J. P., Almouzni, G. SUMOylation promotes de novo targeting of HP1alpha to pericentric heterochromatin. Nat. Genet., 2011. 43: 220-227.

64. Maison, C., Bailly, D., Peters, A. H., Quivy, J. P., Roche, D., Taddei, A., Lachner, M., Jenuwein, T., Almouzni, G. Higher-order structure in pericentric heterochromatin involves a distinct pattern of histone modification and an RNA component, Nat. Genet., 2002. 30: 329-334.

65. Vicent, G.P., Nacht, A. S., Zaurin, R., Font-Mateu, J., Soronellas, D., Le, Dily, F., Reyes, D., Beato, M. Unliganded progesterone receptor-mediated targeting of an RNA-containing repressive complex silences a subset of hormoneinducible genes. Genes dev., 2013. 27: 1179-1197.

66. Guttman, M., Donaghey, J., Carey, B.W., Garber, M., Grenier, J.K., Munson, G., Young, G., Lucas, A.B., Ach, R., Bruhn, L., Yang, X., Amit, I., Meissner, A., Regev, A., Rinn, J.L., Root, D.E., Lander, E.S. lincRNAs act in the circuitry controlling pluripotency and differentiation. Nature, 2011. 477: 295-300.

67. Piacentini, L., Fanti, L., Berloco, M., Perrini, B., Pimpinelli, S. Heterochromatin protein 1 (HP1) is associated with induced gene expression in Drosophila euchromatin. J. cell biol., 2003. 161: 707-714.

68. Keller C., Kulasegaran-Shylini R., Shimada, Y., Hotz, H.R., Buhler, M. Noncoding RNAs prevent spreading of a repressive histone mark. Nat. Stru. Mol.biol., 2013. 20: 994-1000. 
69. Dougherty, E. S., Hansen E. L., Nicholas, W. L., Mollett J. H., Yarwood, E. A. Axenic cultivation of Caenorhabditis elegans (Nematoda: Rhabditidae) with supplemented and unsupplemented chemically defined media. Ann. N.Y. Acad. Sci. 1959. 77: 176-217.

70. Brenner, S. The Genetics of Caenorhabditis elegans Genetics 1974 77(1): 7194.

71. Ellis, H. M., Horvitz, H. R. Genetic control of programmed cell death in the nematode C. elegans. Cell. 1986. 44(6):817-29.

72. Sulston, J. E. and Brenner, S. The DNA of Caenorhabditis elegans. Genetics 1974 77: 95-104.

73. The C. elegans Sequencing Consortium. Genome sequence of the nematode C. elegans: a platform for investigating biology. Science 1998. 282: 20122018.

74. Hillier, L.W. et al. Genomics in C. elegans: so many genes, such a little worm. Genome Res. 2005. 15: 1651-1660.

75. Maniatis, T., Fritsch, E.F., Sambrook, J. Molecular cloning: a laboratory manual. New York: Cold Spring Harbor Laboratory Press. 1982: 68.

76. Cui, M., Han, M. Roles of chromatin factors in C. elegans development. WormBook. 2007: 1-16.

77. Schaner, C.E. Kelly, W. G. Germline chromatin. WormBook. 2006: 1-14.

78. Ikegami, K., Egelhofer, T., Strome, S., Lieb, J. D. C. elegans chromosome arms are anchored to the nuclear membrane via discontinuous association with LEM-2. Genome Biol. 2010 11: R20.

79. Kolasinska-Zwierz, P., et al., Differential chromatin marking of introns and expressed exons by H3K36me3. Nat. Genet. 2009. 41(3): 376-81. 
80. Grant, J., Verrill, C., Coustham, V., Arneodo, A., Palladino, F., Monier, K., Khalil, A. Perinuclear distribution of heterochromatin in developing $C$. elegans embryos. Chromosome Res. 2010.18(8): 873-85.

81. Meister, P., Towbin, B. D., Pike, B. L., Ponti, A., Gasser, S. M. The spatial dynamics of tissue-specific promoters during $C$. elegans development. Genes Dev. 2010. 24(8): 766-82.

82. Gu, S. G., Pak, J., Guang, S., Maniar, J. M., Kennedy, S., Fire, A. Amplification of siRNA in Caenorhabditis elegans generates a transgenerational sequencetargeted histone H3 lysine 9 methylation footprint. Nat. Genet 2012. 44:157-64.

83. Garrigues, J. M., Sidoli, S., Garcia, B. A., Strome, S. Defining heterochromatin in $\mathrm{C}$. elegans through genome-wide analysis of the heterochromatin protein 1 homolog HPL-2. Genome Res. 2015. 25: 76-88.

84. Juang, B. T., Gu, C., Starnes, L., Palladino, F., Goga, A., Kennedy, S., L'Etoile, N. D. Endogenous nuclear RNAi mediates behavioral adaptation to odor. Cell. 2013. 154: 1010-22.

85. Sarkies, P., Miska, E. A. Small RNAs break out: the molecular cell biology of mobile small RNAs Nat. Rev. Mol. Cell Biol. 2014. 15: 525-535.

86. Fay, D. S. and J. Yochem The SynMuv genes of Caenorhabditis elegans in vulval development and beyond. Dev. Biol. 2007. 306(1): 1-9.

87. Sternberg, P. W. Vulval development. WormBook. 2005: p. 1-28.

88. Ferguson, E. L., Sternberg, P. W., Horvitz, H. R. A genetic pathway for the specification of the vulval cell lineages of Caenorhabditis elegans. Nature. 1987. 326(6110): 259-67.

89. Moghal, N. Sternberg, P. W. The epidermal growth factor system in Caenorhabditis elegans. Exp. Cell Res. 2003. 284(1): 150-9.

90. Ferguson, E. L., Horvitz, H. R. Identification and characterization of 22 genes that affect the vulval cell lineages of the nematode Caenorhabditis elegans. Genetics. 1985. 110(1): 17-72. 
91. Horvitz, H. R., Sulston, J. E. Isolation and genetic characterization of cell lineage mutants of the nematode Caenorhabditis elegans. Genetics. 1980. 96(2): 435-54.

92. Hill, R. J., Sternberg, P. W. The gene lin-3 encodes an inductive signal for vulval development in C. elegans. Nature. 1992. 358(6386): 470-6.

93. Beitel, G. J., Clark, S. G. Horvitz, H. R. Caenorhabditis elegans ras gene let-60 acts as a switch in the pathway of vulval induction. Nature. 1990. 348(6301): 503-9.

94. Ferguson, E. L., Horvitz, H. R. The multivulva phenotype of certain Caenorhabditis elegans mutants results from defects in two functionally redundant pathways. Genetics. 1989. 123(1): 109-21.

95. Huang, L. S., Tzou, P., Sternberg, P. W. The lin-15 locus encodes two negative regulators of Caenorhabditis elegans vulval development. Mol. Biol. Cell, 1994. 5(4): 395-411.

96. Melendez, A., Greenwald, I. Caenorhabditis elegans lin-13, a member of the LIN-35 Rb class of genes involved in vulval development, encodes a protein with zinc fingers and an LXCXE motif. Genetics. 2000. 155(3): 1127-37.

97. Koester-Eiserfunke, N., Fischle, W. H3K9me2/3 binding of the MBT domain protein LIN-61 is essential for Caenorhabditis elegans vulva development. PLoS Genet. 2011. 7(3), e1002017-e1002017.

98. Andersen, E. C., Saffer, A. M., Horvitz, H. R. Multiple levels of redundant processes inhibit Caenorhabditis elegans vulval cell fates. Genetics. 2008. 179(4): 2001-12.

99. Harrison, M. M., Lu, X., Horvitz H. R. LIN-61, one of two Caenorhabditis elegans malignant-brain-tumor-repeat-containing proteins, acts with the DRM and NuRD-like protein complexes in vulval development but not in certain other biological processes. Genetics. 2007. 176(1): 255-71.

100. New England Biolabs, l., Catalog and technical reference. 2007:200.

101. Sambrook, J., Russel, D. Molecular Cloning. A laboratory Manual. $3^{\text {rd }}$ ed.2001: Cold Spring Harbor, New York: Cold Spring Harbour press. 
102. Wysocka, J., Identifying novel proteins recognizing histone modifications using peptide pull-down assay. Methods, 2006. 40(4): 339-43.

103. Schott, S., Coustham, V., Simonet, T., Bedet, C., Palladino, F. Unique and redundant functions of C. elegans HP1 proteins in post-embryonic development. Dev. Biol. 2006. 298(1): 176-87.

104. Couteau, F., Guerry, F., Muller, F., Palladino, F. A. heterochromatin protein 1 homologue in Caenorhabditis elegans acts in germline and vulval development. EMBO Rep. 2002. 3(3): 235-41.

105. Wu, X., Shi, Z., Cui, M., Han, M., Ruvkun, G. Repression of germline RNAi pathways in somatic cells by retinoblastoma pathway chromatin complexes PLoS Genet. 2012. 8(3): e1002542.

106. Laemmli, U. K., Cleavage of structural proteins during the assembly of the head of bacteriophage T4. Nature. 1970. 227(5259): 680-5.

107. Gallagher, S.R., One-dimensional SDS gel electrophoresis of proteins. Curr. Protoc. Immunol. 2006. Chapter 8: Unit 84.

108. Stiernagle, T., Maintenance of C. elegans. WormBook, 2006: 1-11.

109. Lewis, J. A. and J. F. Flemming. Basic culture methods, in Methods in Cell Biology Caenorhabditis elegans Modern Biological Analysis of an Organism. E.H.F.a.S. D.C., Editor. 1995.

110. Wysocka, J., Identifying novel proteins recognizing histone modifications using peptide pull-down assay. Methods, 2006. 40(4): 339-43.

111. Duerr, J.S., Immunohistochemistry. WormBook, 2006: 1-61.

112. Troemel, E. R., Chu, S. W., Reinke, V., Lee, S. S., Ausubel, F. M., Kim D. H. p38 MAPK regulates expression of immune response genes and contributes to longevity in C. elegans. PLoS Genet, 2006. 2(11): e183.

113. Dobin, A., Davis, C. A., Schlesinger, F., Drenkow, J., Zaleski, C., Jha, S., Batut, P., Chaisson, M., Gingeras, T. R. STAR: ultrafast universal RNA-seq aligner. Bioinformatics. 2013. 29(1):15-21. 
114. Anders, S., Huber, W. Differential expression analysis for sequence count data Genome Biology. 2010. 11:R106.

115. Kamath, R. S., Ahringer, J. Genome-wide RNAi screening in Caenorhabditis elegans. Methods. 2003. 30(4): 313-21.

116. Poulin, G., et al., Chromatin regulation and sumoylation in the inhibition of Rasinduced vulval development in Caenorhabditis elegans. EMBO J, 2005. 24(14): 2613-23.

117. Coustham, V., Bedet, C., Monier, K., Schott, S., Karali, M., Palladino, F. The C. elegans HP1 homologue HPL-2 and LIN-13 zinc finger protein form a complex implicated in vulval development. Dev. Biol. 2006. 297(2): 308-22.

118. Celniker, S. E., Dillon, L. A., Gerstein, M. B., Gunsalus, K. C., Henikoff, S., Karpen, G. H., Kellis, M., Lai, E. C, Lieb, J. D., MacAlpine, D. M., Micklem, G., Piano, F., Snyder, M., Stein, L., White, K. P., Waterston, R. H. modENCODE Consortium. Unlocking the Secrets of the Genome. Nature. 2009.459 (7249): 927-30.

119. Gerstein, M. B. et al. Integrative Analysis of the Caenorhabditis elegans Genome by the modENCODE Project. Science 2010: 330 (6012): $1775-1787$.

120. Niu, W., Lu, Z. J., Zhong, M., Sarov, M., Murray, J. I., Brdlik, Janette, C. M., Chen, C., Alves, P. E., Preston, et al. Diverse transcription factor binding features revealed by genome-wide ChIP-seq in C. elegans Genome Res. 2010 21: 245-254.

121. Holoch, D., and Moazed, D. RNA-mediated epigenetic regulation of gene expression Nat. Rev. Genet. 2015. 16: 71-84.

122. Ashe, A., Sapetschnig, A., Weick, E. M., Mitchell, J., Bagijn, M. P., Cording, A. C., Doebley, A. L., Goldstein, L. D., Lehrbach, N. J., Le, Pen, J., et al. piRNAs can trigger a multigenerational epigenetic memory in the germline of $\mathrm{C}$. elegans. Cell. 2012. 150: 88- 99.

123. Hediger, F., Gasser, S. M. Heterochromatin protein 1: don't judge the book by its cover! Curr. Opin. Genet. Dev. 2006. 16(2): 143-50. 
124. Jacobs, S. A., Taverna, S. D., Zhang, Y., Briggs,S. D., Li, J., Eissenberg, J. C., Allis, C. D., Khorasanizadeh, S. Specificity of the HP1 chromo domain for the methylated N-terminus of histone H3. EMBO J. 2001. 20: 5232-5241.

125. Jacobs, S. A., Khorasanizadeh S. Structure of HP1 chromodomain bound to a lysine 9-methylated histone H3 tail. Science, 2002. 295: 2080-2083.

126. Studencka, M., Konzer, A., Moneron, G., Wenzel, D., Opitz, L., Salinas-Riester, G., Bedet, C., Krüger, M., Hell, S. W., Wisniewski, J. R., Schmidt, H., Palladino, F., Schulze, E., Jedrusik-Bode, M. Novel roles of Caenorhabditis elegans heterochromatin protein HP1 and linker histone in the regulation of innate immune gene expression. Mol. Cell Biol. 2012. 32(2): 251-65.

127. Wirth, M., Paap, F., Fischle, W., Wenzel, D., Agafonov, D. E., Samatov, T. R., Wisniewski, J. R., Jedrusik-Bode, M. HIS-24 linker histone and SIR-2.1 deacetylase induce $\mathrm{H} 3 \mathrm{~K} 27 \mathrm{me} 3$ in the Caenorhabditis elegans germ line. Mol. Cell Biol. 2009. 29(13): 3700-9.

128. Studencka, M., Wesołowski, R., Opitz, L., Salinas-Riester, G., Wisniewski, J. R., Jedrusik-Bode, M. Transcriptional repression of Hox genes by C. elegans HP1/HPL and H1/HIS-24. PLoS Genet. 2012. 8(9): e1002940.

129. Andersen, E C., Horvitz, H. R. Two C. elegans histone methyltransferases repress lin-3 EGF transcription to inhibit vulval development. Development. 2007. 134(16): 2991-9.

130. LeRoy, G., Weston, J. T., Zee, B. M., Young, N. L., Plazas-Mayorca, M. D., Garcia B. A. Heterochromatin protein 1 is extensively decorated with histone code-like post-translational modifications. Mol. Cell Proteomics. 2009. 8: 24322442.

131. Zhao T., Heyduk T., Eissenberg J. C. Phosphorylation site mutations in heterochromatin protein 1 (HP1) reduce or eliminate silencing activity. J. Biol. Chem. 2001. 276: 9512-9518.

132. Ayoub, N., Jeyasekharan, A. D., Bernal, J. A., Venkitaraman, A. R. HP1-ß mobilization promotes chromatin changes that initiate the DNA damage response. Nature 2008. 453: 682-686.

133. Lomberk, G., Bensi, D., Fernandez-Zapico, M. E., Urrutia, R. Evidence for the existence of an HP1-mediated subcode within the histone code. Nature Cell Biol. 2006 8: 407-415. 
134. Thiru, A, Nietlispach, D., Mott, H. R., Okuwaki, M., Lyon, D., Nielsen, P. R., Hirshberg, M., Verreault, A., Murzina, N. V., Laue, E. D. Structural basis of HP1/PXVXL motif peptide interactions and HP1 localisation to heterochromatin. EMBO J. 2004. 23: 489-499.

135. Cowieson, N. P., Partridge, J. F., Allshire, R. C., McLaughlin, P. J. Dimerisation of a chromo shadow domain and distinctions from the chromodomain as revealed by structural analysis. Curr. Biol. 2000 10: 517-525.

136. Li, Y., Kirschmann, D. A., Wallrath, L. L. Does heterochromatin protein 1 always follow code? Proc. Natl. Acad. Sci. 2002. 99: 16462-16469.

137. Nielsen, A. L., Sanchez, C., Ichinose, H., Cervino, M., Lerouge, T., Chambon, $\mathrm{P}$., Losson, R. Selective interaction between the chromatin-remodeling factor BRG1 and the heterochromatin-associated protein HP1a. EMBO J. 2002. 21: 5797-5806.

138. Yamamoto, K., Sonoda, M. Self-interaction of heterochromatin protein 1 is required for direct binding to histone methyltransferase, SUV39H1. Biochem. Biophys. Res. Commun. 2003. 301: 287-292.

139. Eiserfunke NK. Characterization of LIN-61 methyl mark binding and its function in C. elegans vulva development. 2010. PhD thesis.

140. Trojer P, Li, G, Sims R. J., III, et al. L3MBTL1, a histone-methylationdependent chromatin lock. Cell. 2007. 129: 915-928.

141. Kalakonda, N., Fischle, W., Boccuni, P., Gurvich, N., Hoya-Arias, R., Zhao, X., Miyata, Y., Macgrogan, D., Zhang, J., Sims, J. K., Rice, J. C., Nimer, S. D. Histone $\mathrm{H} 4$ lysine 20 monomethylation promotes transcriptional repression by L3MBTL1. Oncogene. 2008. 27(31): 4293-304.

142. Li, H., Fischle, W., Wang, W., Duncan, E. M., Liang, L., Murakami-Ishibe, S., Allis, C. D., Patel, D. J. Structural basis for lower lysine methylation statespecific readout by MBT repeats of L3MBTL1 and an engineered PHD finger. Mol. Cell. 2007. 28(4): 677-91.

143. Klug, A. Zinc finger peptides for the regulation of gene expression. J. Mol. Biol. 1999. 293: 215-218. 
144. Brown, R. S. Zinc finger proteins: getting a grip on RNA. Curr. Opin. Struct. Biol. 2005. 15: $94-98$.

145. Gamsjaeger, R., Liew, C. K., Loughlin, F. E., Crossley, M., Mackay, J. P. Sticky fingers: zinc-fingers as protein-recognition motifs. Trends. Biochem. Sci. 2007. 32: $63-70$.

146. Matthews, J. M., Sunde, M. Zinc fingers-Folds for many occasions. IUBMB Life. 2002. 54: 351-355.

147. Fukushige, T., Hendzel, M. J., Bazett-Jones, D. P., McGhee, J. D., Direct visualization of the elt-2 gut-specific GATA factor binding to a target promoter inside the living Caenorhabditis elegans embryo. Proc. Natl. Acad. Sci. 1999. 96: $11883-11888$.

148. Kamath, R. S. and Ahringer, J. Genome-wide RNAi screening in Caenorhabditis elegans. Methods. 2003. 30(4): 313-21.

149. Lee, J. O., Russon, A. A., Pavletich, N. P. Structure of the retinoblastoma tumour-suppressor pocket domain bound to a peptide from HPV E7. Nature 1998. 391: 859-865.

150. Schott, S., Ramos, F., Coustham, V., Palladino, F. HPL-2/HP1 prevents inappropriate vulval induction in Caenorhabditis elegans by acting in both HYP7 and vulval precursor cells. Genetics. 2009. 181(2): 797-801.

151. A. M. Saffer, D. H. Kim, Oudenaarden, A., van, Horvitz H. R.The Caenorhabditis elegans synthetic multivulva genes prevent ras pathway activation by tightly repressing global ectopic expression of lin-3 EGF. PLoS Genet. 2011. 7. e1002418.

152. Cui, M., Chen, J., Myers, T. R., Hwang, B. J, Sternberg, P. W., Greenwald, I, Han, M. SynMuv genes redundantly inhibit lin-3/EGF expression to prevent inappropriate vulval induction in C. elegans. Dev. Cell. 2006. 10(5): 667-72.

153. Platero, J. S., Harnett, T., Eissenberg, J. C. Functional analysis of the chromodomain of HP-1. EMBO J. 1995. 14: 3977-3986.

154. Lachner, M., O'Carroll, D., Rea, S., Mechtler, K., Jenuwein, T. Methylation of histone $\mathrm{H} 3$ lysine 9 creates a binding site for HP1 proteins. Nature. 2001. 410 : 116-120. 
155. Ekwall, K., Nimmo, E. R., Javerzat, J. P., Borgstrøm, B., Egel, R., Cranston, G., Allshire, R. Mutations in the fission yeast silencing factors clr4+ and rik1+ disrupt the localisation of the chromo domain protein Swi6p and impair centromere function. J. Cell Sci. 1996. 109: 2637-2648.

156. Choi, J., Newman, A. P. A two-promoter system of gene expression in C. elegans. Dev. Biol. 2006. 296: 537-544.

157. Fuxman, Bass, J.I., Tamburino, A. M., Mori, A., Beittel, N., Weirauch, M. T., Reece-Hoyes, J. S., Walhout, A. J. Transcription factor binding to Caenorhabditis elegans first introns reveals lack of redundancy with gene promoters. Nucleic Acids Res. 2014. 42: 153-162.

158. Weintraub, H., Groudine M. Chromosomal subunits in active genes have an altered conformation. Science. 1976. 193: 848-856.

159. Bird, A. DNA methylation patterns and epigenetic memory. Genes Dev. 2002. 16: 6-21.

160. Macleod, D., Ali, R. R., Bird, A. An alternative promoter in the mouse major histocompatibility complex class II I-A beta gene: implications for the origin of CpG islands. Mol. Cell. Biol. 1998. 18: 4433-4443.

161. Herman, J. G., Jen, J., Merlo, A., Baylin, S., B. Hypermethylation-associated inactivation indicates a tumor suppressor role for p15INK4B Cancer Res. 1996. 56: $722-727$.

162. Okano, M., Xie, S., Li, E. Cloning and characterization of a family of novel mammalian DNA (cytosine-5) methyltransferases. Nat. Genet. 1998. 19:219220.

163. Okano, M., Bell, D. W., Haber, D.A., Li, E. DNA methyltransferases Dnmt3a and Dnmt3b are essential for de novo methylation and mammalian development. Cell. 1999. 99:247-257.

164. Hermann, A., Schmitt, S., Jeltsch, A. The Human Dnmt2 has residual DNA (cytosine-C5) methyltransferase activity. J. Biol. Chem. 2003. 278: 3171731721.

165. Wu, S. C., Zhang, Y. Active DNA demethylation: many roads lead to Rome. Nat. Rev. Mol. Cell. Biol. 2010. 11: 607-620. 
166. Bhutani, N., Burns, D. M. Blau, H. M. DNA demethylation dynamics. Cell. 2011. $146866-872$.

167. Hendrich, B., Tweedie, S. The methyl-CpG binding domain and the evolving role of DNA methylation in animals. Trends Genet. 2003. 19: 269-277.

168. Prokhortchouk, A., Hendrich, B., Jorgensen, H., Ruzov, A., Wilm, M., Georgiev, G., Bird, A., Prokhortchouk E. The p120 catenin partner Kaiso is a DNA methylation dependent transcriptional repressor. Genes Dev. 2001. 15: 16131618.

169. Marzluff, W. F., Gongidi, P., Woods, K. R., Jin, J., Maltais, L. J. The human and mouse replication-dependent histone genes. Genomics. 2002. 80(5): 487-498.

170. Albig, W., Doenecke, D. The human histone gene cluster at the D6S105 locus. Hum. Genet. 1997. 101:284-294.

171. Tagami, H., Ray-Gallet, D., Almouzni, G., Nakatani, Y. Histone H3.1 and H3.3 complexes mediate nucleosome assembly pathways dependent or independent of DNA synthesis. Cell. 2004. 116(1): 51-61.

172. Heo, K., Kim, H., Choi, S. H., Choi, J., Kim, K., Gu, J., Lieber, M. R., Yang, A. S., An, W. FACT-mediated exchange of histone variant $\mathrm{H} 2 \mathrm{AX}$ regulated by phosphorylation of H2AX and ADP-ribosylation of Spt16. Mol Cell. 2008. 30(1): 86-97.

173. Chakravarthy, S., Gundimella, S. K, Caron, C., Perche, P. Y., Pehrson, J. R., Khochbin, S., Luger, K. Structural characterization of the histone variant macroH2A. Mol. Cell. Biol. 2005. 25:7616-7624.

174. Thakar, A., Gupta, P., Ishibashi, T., Finn, R., Silva-Moreno, B., Uchiyama, S., Fukui, K., Tomschik, M., Ausio, J., Zlatanova, J. H2A.Z and H3.3 histone variants affect nucleosome structure: biochemical and biophysical studies. Biochemistry. 2009. 48(46): 10852-10857.

175. Yoda, K., Ando, S., Morishita, S., Houmura, K., Hashimoto, K., Takeyasu, K., Okazaki, T. Human centromere protein A (CENP-A) can replace histone H3 in nucleosome reconstitution in vitro. Proc. Natl. Acad. Sci. 2000. 97:7266-7271.

176. Rogakou, E. P., Pilch, D. R., Orr, A. H., Ivanova, V. S., Bonner, W. M. DNA double stranded breaks induce histone H2AX phosphorylation on serine 139. J 
Biol. Chem. 1998. 273:5858-5868.

177. Clapier, C. R. and Cairns, B. R. The Biology of Chromatin Remodeling Complexes. Annu. Rev. Biochem. 2009. 78: 273-304.

178. Ryan, M. P., Jones, R., Morse, R. H. SWI-SNF Complex Participation in Transcriptional Activation at a Step Subsequent to Activator Binding. Mol. Cell. Biol. 1998. 18(4): 1774-1782.

179. Racki1, L. R., Yang, J. G., Naber, N., Partensky1, P. D., Acevedo1, A., Purcell1, T. J., Cooke1, R., Cheng Y., Narlikar G. J. The chromatin remodeller ACF acts as a dimeric motor to space nucleosomes. Nature. 2009. 462: 10161021.

180. Marfella C. G. A. and Imbalzano, A. N. The Chd Family of Chromatin Remodelers. Mutat. Res. 2007. 618(1-2): 30-40.

181. Carninci P, Hayashizaki Y: Noncoding RNA transcription beyond annotated genes. Curr. Opin. Genet. Dev. 2007. 17: 139-144.

182. Kapranov, P., Cheng, J., Dike, S., Nix, D. A., Duttagupta, R., Willingham, A. T., Stadler, P. F., Hertel, J., Hackermüller, J., Hofacker, I. L. et al. RNA maps reveal new RNA classes and a possible function for pervasive transcription. Science. 2007. 316:1484-1488.

183. Maenner, S., Blaud, M., Fouillen, L., Savoye, A., Marchand, V., Dubois, A., Sanglier-Cianfe'rani, S., Van, Dorsselaer, A., Clerc, P., Avner, P. et al. 2-D structure of the A region of Xist RNA and its implication for PRC2 association PLoS Biol. 2010. 8:e1000276.

184. Tsai M. C., Manor, O., Wan, Y., Mosammaparast, N., Wang, J. K., Lan F., Shi, Y., Segal, E., Chang H. Y. Long noncoding RNA as modular scaffold of histone modification complexes. Science. 2010. 329(5992): 689-693.

185. Guang S, Bochner, A. F., Burkhart, K. B., Burton, N., Pavelec, D. M., Kennedy, S. Small regulatory RNAs inhibit RNA polymerase II during the elongation phase of transcription. Nature. 2010. 465(7301): 1097-1101.

186. Birney, E. et al. Identification and analysis of functional elements in $1 \%$ of the human genome by the ENCODE pilot project. Nature. 2007. 447: 799-816. 
187. The ENCODE project consortium. An integrated encyclopedia of DNA elements in the human genome. Nature. 2012. 489: 57-74.

188. Tessarz P, Kouzarides T. Histone core modifications regulating nucleosome structure and dynamics. Nat. Rev. Mol. Cell. Biol. 2014. 15(11): 703-8.

189. Simon, M. et al. Histone fold modifications control nucleosome unwrapping and disassembly. Proc. Natl. Acad. Sci. 2011. 108: 12711-12716.

190. Casadio, F., Lu, X., Pollock, S. B., LeRoy, G. H3R42me2a is a histone modification with positive transcriptional effects. Proc. Natl. Acad. Sci. 2013. 110: 14894-14899.

191. Iwasaki, W. et al. Comprehensive structural analysis of mutant nucleosomes containing lysine to glutamine (KQ) substitutions in the $\mathrm{H} 3$ and $\mathrm{H} 4$ histone-fold domains. Biochemistry. 2011. 50: 7822-7832.

192. North, J. A. et al. Histone H3 phosphorylation near the nucleosome dyad alters chromatin structure. Nucleic Acids Res. 2014. 42: 4922-4933.

193. Arnaudo A. M., Garcia B. A. Proteomic characterization of novel histone posttranslational modifications. Epigenetics \& Chromatin. 2013. 6:24.

194. Smothers J. F., Henikoff, S. The hinge and chromo shadow domain impart distinct targeting of HP1-like proteins. Mol. Cell Biol. 2001. 21(7): 2555-69.

195. Munro, S., Khaire, N., Inche, A., Carr, S., La, Thangue. Lysine methylation regulates the $\mathrm{pRb}$ tumour suppressor protein. Oncogene. 2010. 29: 2357-2367.

196. Guil, S., Soler, M., Portela, A., Carrère, J., Fonalleras, E., Gómez, a., Villanueva, A., Esteller, M. Intronic RNAs mediate EZH2 regulation of epigenetic targets. Nat. Struc. Mol. Biol. 2012. 19: 664-670.

197. Harshman, S. W., Young, N., L., Parthun, M.R., Freitas, M.A. H1 histones: Current perspectives and challenges. Nucleic Acids Res. 2013. 41(21): 95939609.

198. T. Pederson. Chromatin structure and the cell cycle. Proc. Natl. Acad. Sci. 1972. 69: 2224-2228.

199. Bak, A. L., Zeuthen, J, Crick H. Higher-order structure of human mitotic chromosomes. Proc. Natl. Acad. Sci. 1977. 74:1595-1599. 
200. Robinson, P. J., Fairall, L., Huynh, V. A., Rhodes D. EM measurements define the dimensions of the "30-nm" chromatin fiber: evidence for a compact, interdigitated structure. Proc Natl Acad Sci. 2006. 103: 6506-6511.

201. Maeshima K., Hihara S., Eltsov M. Chromatin structure: does the 30-nm fibre exist in vivo. Curr. Opin. Cell Biol. 2010. 22:291-297.

202. Elgin, S.C. "Heterochromatin and gene regulation in Drosophila". Curr. Opin Genet. Dev. 1996. 6 (2): 193-202.

203. Grewal S., Jia, S. "Heterochromatin revisited". Nature Rev. Genet. 2007. 8 (1): 35.

204. Elgin, S. C., Reuter, G. Position-effect variegation, heterochromatin formation, and gene silencing in Drosophila. Cold Spring Harb. Perspect. Biol. 2013. 5, a017780

205. Eissenberg, J. C., Morris, G. D., Reuter, G., Hartnett. T. "The heterochromatinassociated protein HP-1 is an essential protein in Drosophila with dosagedependent effects on position-effect variegation". Genetics 1992. 131:345-352.

206. Waddington, C.H., Epigenetics and evolution. Symp Soc Exp Biol, 1953. 7: 186-199.

207. Smolle, M., Workman, J. L., Transcription-associated histone modifications and cryptic transcription, Biochim. Biophys. Acta. 2013. 1829:84-97.

208. Dhalluin, C., Carlson J. E., Zeng, L., He, C., Aggarwal, A. K., Zhou, M. M. Structure and ligand of a histone acetyltransferase bromodomain. Nature 1999; 399:491-496.

209. Mujtaba, S., Zeng L., Zhou M. M. Structure and acetyl-lysine recognition of the bromodomain. Oncogene. 2007. 26: 5521-5527.

210. Gelato, K. A., Tauber, M., Ong, M. S., Winter, S., Hiragami-Hamada, K., Sindlinger, J., Lemak, A., Bultsma, Y., Houliston, S., Schwarzer, D., Divecha, N., Arrowsmith, C. H., Fischle W. Accessibility of different histone H3-binding domains of UHRF1 is allosterically regulated by phosphatidylinositol 5phosphate. Mol. Cell. 2014. 54(6):905-19.

211. Walter, W., Clynes, D., Tang, Y., Marmorstein, R., Mellor, J., Berger, S. L. 14- 
3-3 interaction with histone $\mathrm{H} 3$ involves a dual modification pattern of phosphoacetylation. Mol Cell Biol 2008; 28:2840-2849.

212. Ruthenburg, A. J., Li, H., Milne, T. A., Dewell, S., McGinty, R. K., Yuen, M., Ueberheide, B., Dou, Y., Muir, T. W., Patel, D. J., Allis, C. D. Recognition of a mononucleosomal histone modification pattern by BPTF via multivalent interactions. Cell. 2011. 145(5): 692-706.

213. Joshi AA, Struhl K Eaf3 chromodomain interaction with methylated H3-K36 links histone deacetylation to Pol II elongation. Mol Cell. 2005. 20:971-978.

214. Zhao, T., Heyduk, T., Allis, C. D., Eissenberg, J. C. Heterochromatin protein 1 binds to nucleosomes and DNA in vitro. J. Biol. Chem. 2000. 275:2833228338.

215. Thomas, M. Guérin, Palladino, F., Robert V. J. Transgenerational functions of small RNA pathways in controlling gene expression in C. elegans. Epigenetics. 2014. 9(1): 37-44.

216. Ernst, J., Kellis, M. Discovery and characterization of chromatin states for systematic annotation of the human genome. Nat Biotechnol. 2010. 28:817825.

217. Wang, Z., Zang, C., Rosenfeld, J. A., Schones, D. E., Barski, A., Cuddapah, S., Cui, K., Roh, T. Y., Peng, W., Zhang, M. Q., Zhao, K. Combinatorial patterns of histone acetylations and methylations in the human genome. Nat Genet. 2008. 40:897-903.

218. Davison, E. M., Saffer, A. M., Huang, L. S., DeModena, J., Sternberg, P. W., Horvitz, H. R. The LIN-15A and LIN-56 transcriptional regulators interact to negatively regulate EGF/Ras signaling in Caenorhabditis elegans vulval cellfate determination. Genetics. 2011. 187: 803-15.

219. Jacobs, S. A., Fischle, W., and Khorasanizadeh, S. Assays for the determination of structure and dynamics of the interaction of the chromodomain with histone peptides. Methods Enzymol. 2004. 376: 131-148.

220. Kudron, M, Niu, W., Lu, Z., Wang, G., Gerstein, M., Snyder, M., Reinke, V. Tissue-specific direct targets of Caenorhabditis elegans Rb/E2F dictate distinct somatic and germline programs. Genome Biol. $2013.214(1)$. 


\section{Acknowledgements}

I would like to thank everyone who has contributed in shaping this thesis. I express my sincere gratitude towards Prof. Dr. Wolfgang Fischle for providing me an opportunity to work in his laboratory and for his constant support. His creativity, passion, enthusiasm and approach towards 'chromatin problems' have and will always be a source of inspiration for me.

I thank my thesis committee members Dr. Dieter Klopfenstein, Prof. Herbert Jaeckle and Prof. Michael Kessel for their valuable time and stimulating discussions during the committee meetings. I also thank Prof. Joerg Grosshans and Prof. Sigrid Hoyer-Fender to be part of the thesis examination board.

I would like to thank all the current and past members of Fischle group. I sincerely thank Dr. Chen Zheng for initial set-up of $C$. elegans methods, Dr. Nora Eiserfunke and Lena Hyatt for their invaluable contribution to the project. I also thank Dr. Alexandra Stuetzer for FP measurements, generous support and advice in the lab. Special thanks goes to Lydia Abdelhalim for providing daily support to work in the lab. I would like to thank Sebastian David for his smart questions (well, elephant is biggest land animal in the world!). I thank Elizabeth Miller not only for her $C$. elegans skills and MSc thesis work, but also for teaching me why fat cats are cuter than everything else and how the bunny runs! I thank Monika for her enthusiasm to continue the project further. I thank Natalie, Nenja, Lisa and Aaron who have helped at various stages by making $C$. elegans recipes.

I sincerely thank my collaborators Orr Shomroni, Vincenzo Capace, Dr. Magalli Hennion and Dr. Stefan Bonn for their indispensable bioinformatics support. I also thank Prof. Francesca Palladino, Prof. Gary Ruvkun and Prof. Robert Horvitz for sharing $C$. elegans strains. 
I thank GGNB office for constant support, helpful methods courses and refreshing retreats. I also thank Frau Viera Grasshoff for improving my German skills, which made my stay in Germany way easier.

I sincerely thank all my master's Professors for their excellent training. The roots of my interest in chromatin go back to the course on 'Transcription' by Prof. Saroj Ghaskadbi. I thank Anand, Ayush, Hari, Rakesh, Rohan, Salma, Shilpee, Simi and Vijay for their scientific discussions and friendship.

Lastly, I would like to thank my parents who have always been an immense source of inspiration, my wife Sarika, my brother Praful and entire extended family for their unconditional support. 


\section{CURRICULUM VITAE}

\section{Personal details:}

Name:

Yogesh Bhojraj Ostwal

Date of birth: 26/05/1986

Place of birth:

Ahmednagar, India

Nationality:

India

\section{Schooling:}

1989-01: $\quad$ S.S.V. School, Maharashtra State Board

2001-03: HSC, S.P.College, Maharashtra State Board

2003-07: Bachelors in Biotechnology, Modern College, University of Pune

Studies:

2007-09: M.Sc. Department of Zoology, university of Pune

2011-2015: GGNB: Genes and Development PhD program in University of Goettingen. PhD student at Max-Planck Institute of Biophysical Chemistry, Goettingen. Thesis: Regulation of HP1 binding to Chromatin and its implications in C. elegans vulva development

\section{Research experience:}

2009-11: Research scholarship by Council of Scientific and Industrial Research (CSIR), India. Project: Role of CARM1-mediated H3R17me2a in oral cancer manifestation

2011-2015 Max-Planck Institute, stipend

\section{List of Publications:}

1. Kost N, Kaiser S, Ostwal Y, Riedel D, Stützer A, Nikolov M, Rathke C, Renkawitz-Pohl R, Fischle W. (2015) Multimerization of Drosophila sperm protein Mst77F causes a unique condensed chromatin structure. Nucleic Acids Res. 43(6): 3033-45 
2. Wilkins BJ, Rall NA, Ostwal Y, Kruitwagen T, Hiragami-Hamada K, Winkler M, Barral Y, Fischle W, Neumann H (2014). A cascade of histone modifications induces chromatin condensation in mitosis. Science. 343(6166): 77-80

3. Ostwal YB, Senapati P and Kundu TK (2010) Epigenetics and Cancer. Society of biological chemists, newsletter, India Review

4. Arif M, Vedamurthy BM, Chaudhari R, Ostwal YB, Montelingu K, Kodaganur GS and Kundu TK (2010). Nitric oxide-mediated histone hyperacetylation in oral cancer: target for a water-soluble HAT inhibitor, CTK7A. Chem. Biol. 17: 903-13

5. Selvi BR, Mohankrishna DV, Ostwal YB and Kundu TK (2010) Small molecule modulators of histone acetylation and methylation: a disease perspective. Biochim Biophys Acta 1799: 810-28 Review 